\title{
HAPSS
}

\section{Hybrid Aircraft Propulsion System Synthesis}

\author{
A Thesis \\ presented to \\ the Faculty of California Polytechnic State University, \\ San Luis Obispo
}

\author{
In Partial Fulfillment \\ of the Requirements for the Degree \\ Master of Science in Aerospace Engineering \\ by \\ Michael W. Green \\ June 2012
}


(c) 2012

Michael W. Green

ALL RIGHTS RESERVED 


\section{COMMITTEE MEMBERSHIP}

TITLE:

AUTHOR:

DATE SUBMITTED:

COMMITTEE CHAIR:

COMMITTEE MEMBER:

COMMITTEE MEMBER:
HAPSS, HYBRID AIRCRAFT PROPULSION SYSTEM SYNTHESIS

Michael W. Green

June 2012
Dr. Dianne J. DeTurris

Advisor and Committee Chair

Aerospace Engineering Dept.

Dr. John Dunning

Committee Member

Mechanical Engineering Dept.

Dr. William W. Durgin

Committee Member

State University of New York Institute of Technology

Benjamin Schiltgen

Committee Member

Empirical Systems Aerospace, Inc. 


\section{ABSTRACT \\ HAPSS, HYBRID AIRCRAFT PROPULSION SYSTEM SYNTHESIS \\ Michael W. Green}

Hybrid Aircraft Propulsion System Synthesis (HAPSS) is a computer program that sizes and analyzes pure-series hybrid electric propulsion systems for aircraft. The development of this program began during a NASA SBIR contract, in conjunction with Empirical Systems Aerospace (ESAero), with the creation of a propulsion fan design tool. Since the completion of this contract in July 2010, the HAPSS program has been expanded to combine the many aspects of a hybrid propulsion system such as the propulsive fans, electric motors, generators, and controllers, and the internal combustion engines.

This thesis describes the benefits and drawbacks of aircraft hybrid propulsion systems to reveal the usefulness of a program of this nature. The methodology behind HAPSS, the creation of the program, its operation, and its many applications are also discussed in detail. Finally, this thesis includes a brief example in which HAPSS is used to analyze a hybrid propulsion system for a commercial transport aircraft. This example demonstrated the usefulness of the program and revealed interesting behavior and trends unique to hybrid propulsion.

To date, the HAPSS program has been utilized on several different contract projects in which an aircraft hybrid propulsion system was designed. In the summer of 2012, a government organization in conjunction with ESAero will begin funding a contract to continue the development of HAPSS by adding functionality and improving accuracy while making the tool available to other government agencies. 


\section{ACKNOWLEDGEMENTS}

Many thanks go to the staff at ESAero for allowing me to conduct my thesis work using their NASA SBIR project as a starting point. Without them, this project would not have taken off so quickly and with such a defined direction as it did. Ben Schiltgen, a Cal Poly graduate and aerospace engineer at ESAero, has been a technical advisor on this project since its inception in early 2010. He has spent countless hours using the program while providing constructive feedback and support.

Mark Waters, the original technical advisor on this project, passed away in March, 2010. He stood by me during my year-long senior project, double-checking results, providing relevant literature, and proofreading documents. He has been an outstanding mentor to myself and others, and I view him as a father figure and someone whom I aspire to one day be. The notion of HAPSS originally belonged to Mr. Waters and I was employed to help him create it. After his passing, Mr. Schiltgen and ESAero took over Mr. Waters' role as technical advisor and oversaw the completion of the program without abandoning the concept originally envisioned by Mr. Waters.

I also would like to thank Dr. Dianne DeTurris for being an exceptional advisor for this thesis. At times, this project appeared to lose direction and focus, but Dr. DeTurris always provided support and asked the necessary questions for me to refocus my thoughts and get back on the correct path. 


\section{Table of Contents}

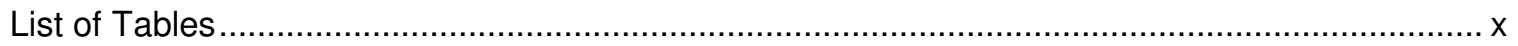

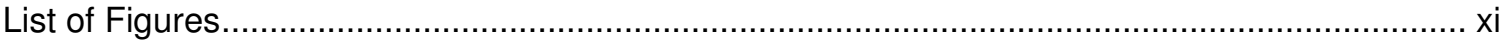

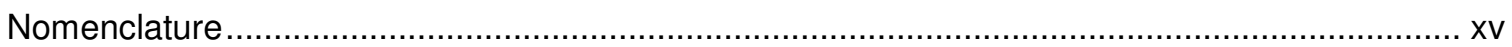

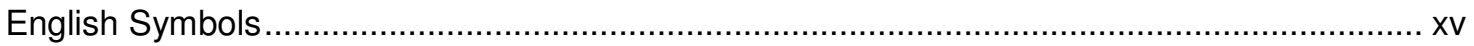

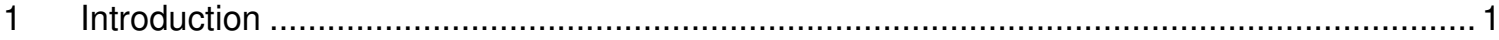

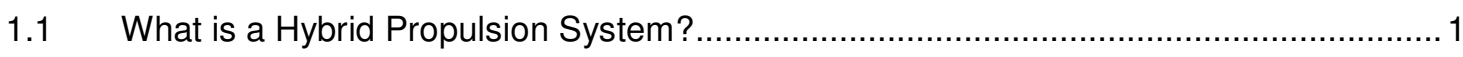

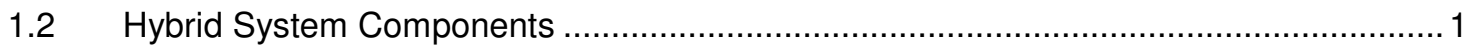

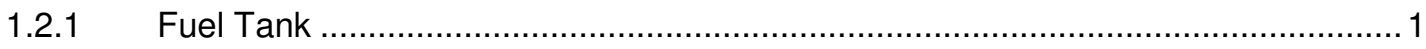

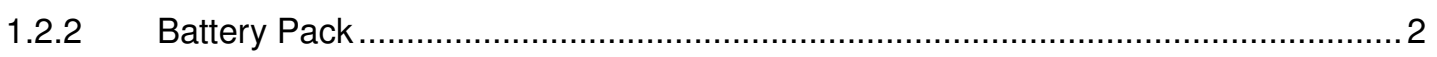

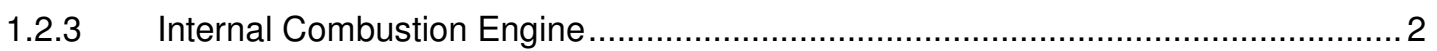

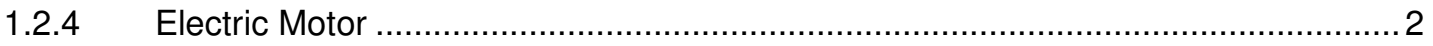

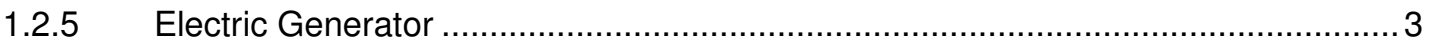

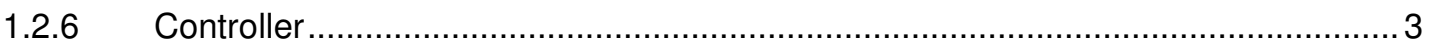

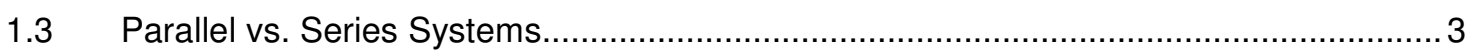

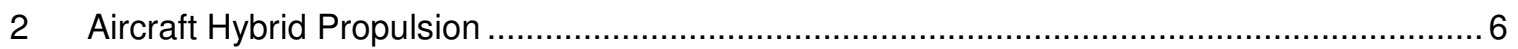

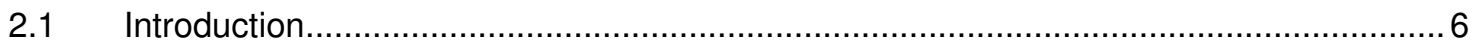

2.2 Series vs. Parallel Systems for Aircraft.................................................................... 7

2.3 Advantages of Hybrid Aircraft Propulsion .................................................................. 10

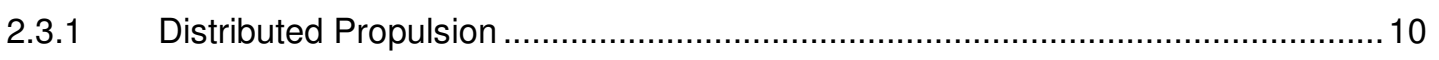

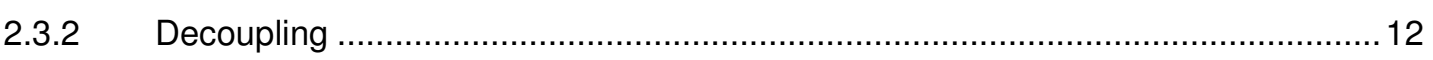

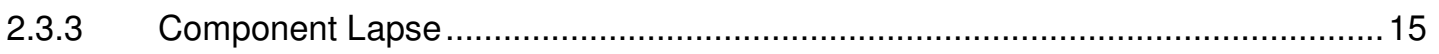

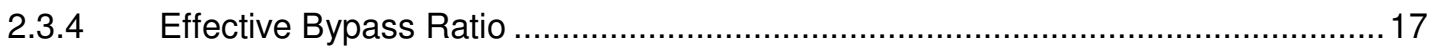

2.3.5 Aircraft Control \& Transients ...................................................................... 18

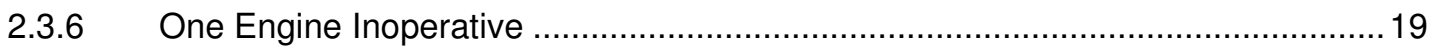

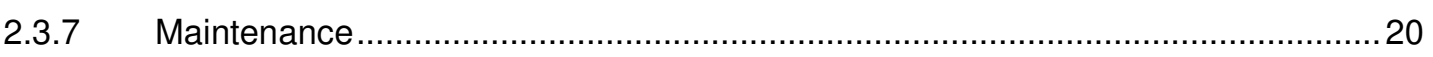

2.3.8 Descent Power \& Windmilling ................................................................ 20

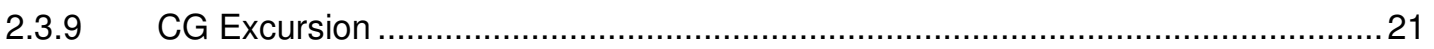

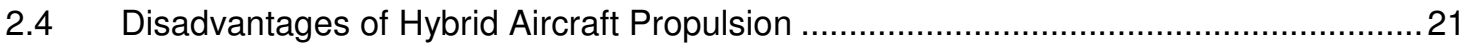

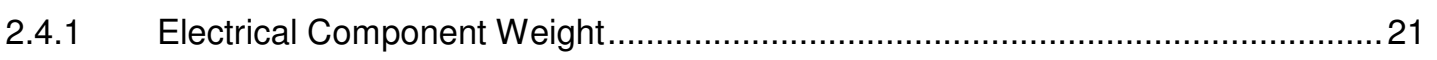

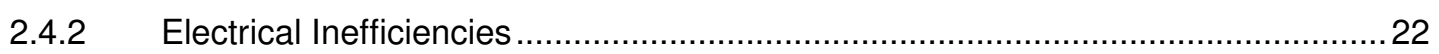

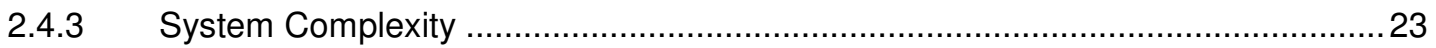

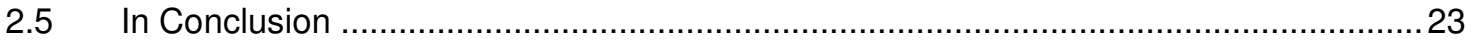

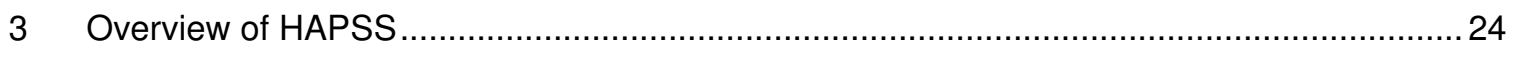

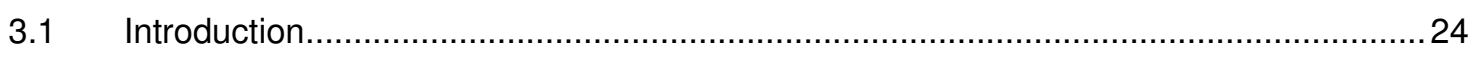

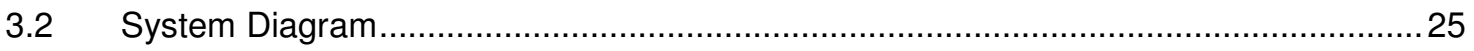




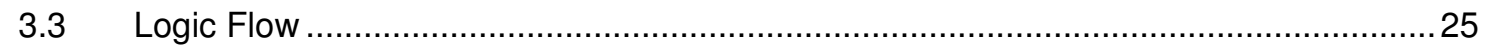

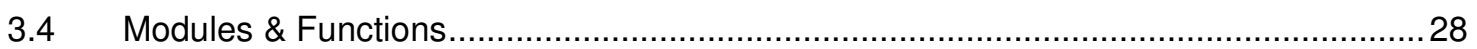

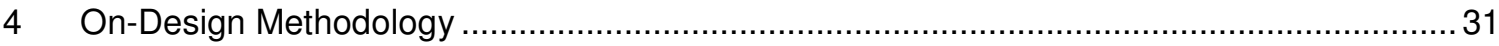

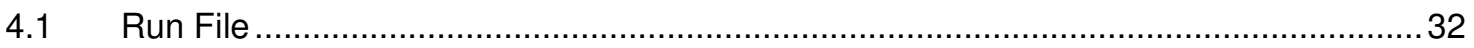

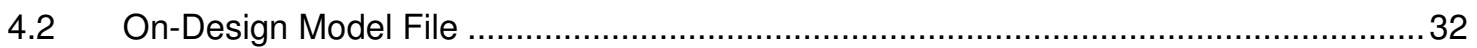

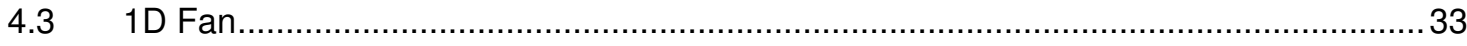

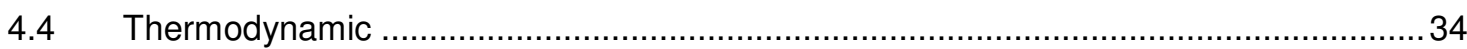

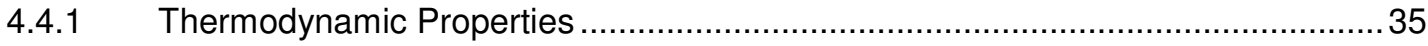

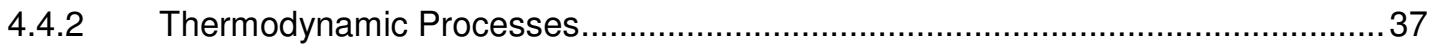

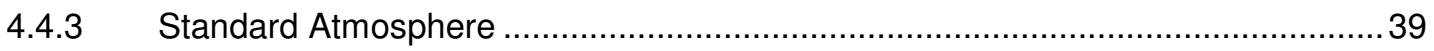

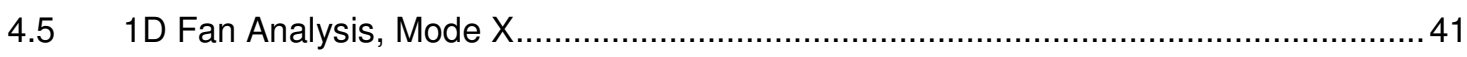

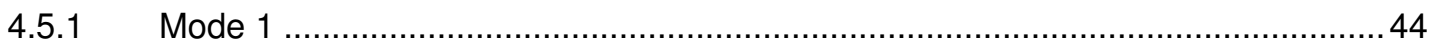

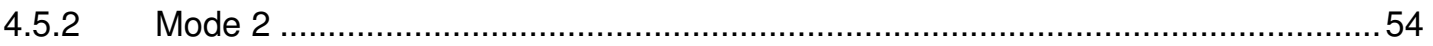

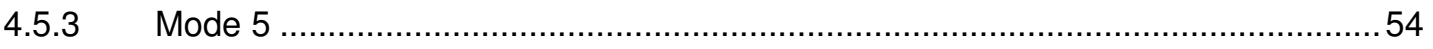

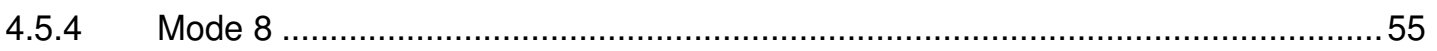

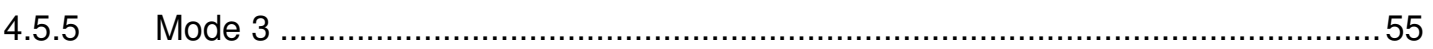

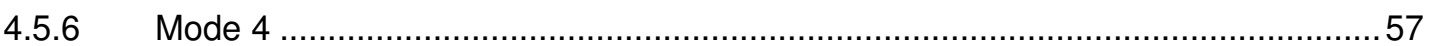

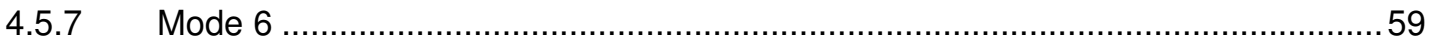

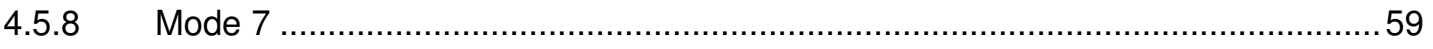

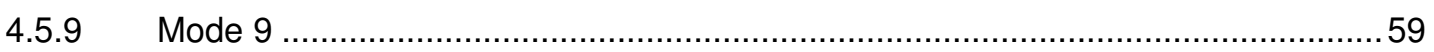

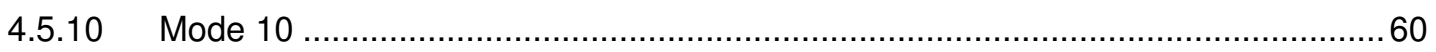

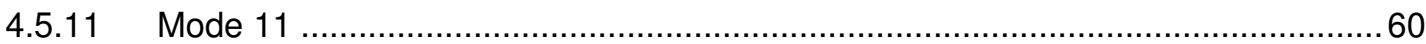

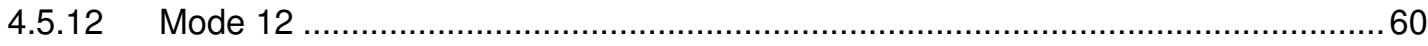

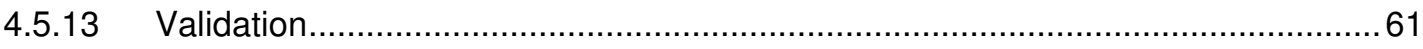

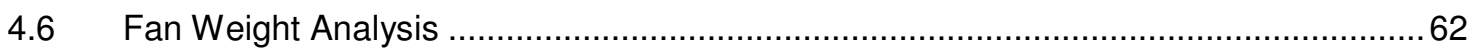

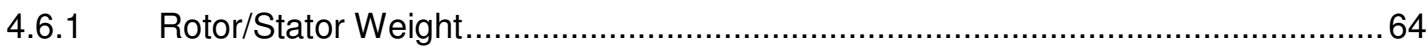

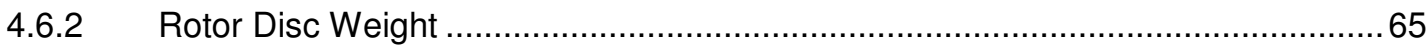

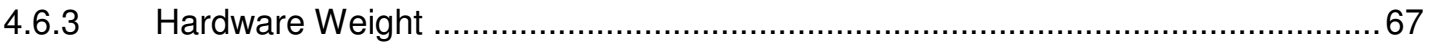

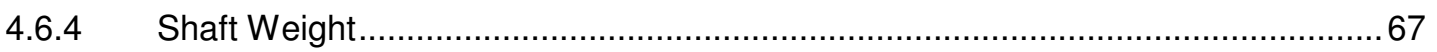

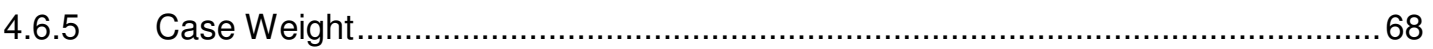

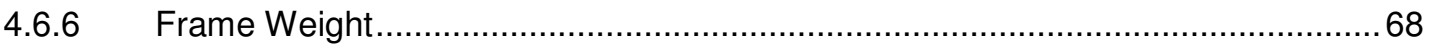

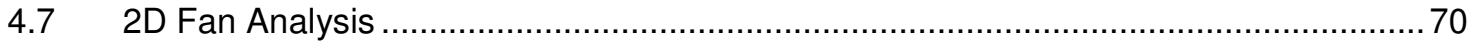

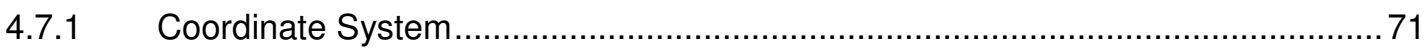

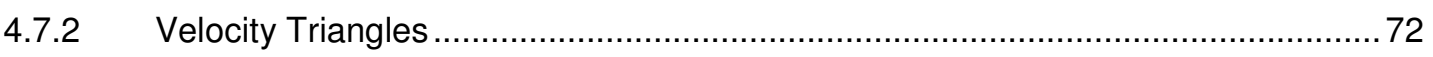

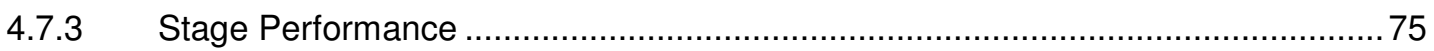

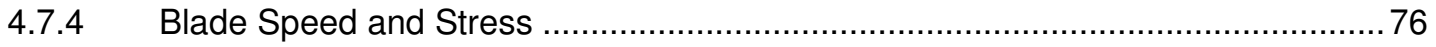

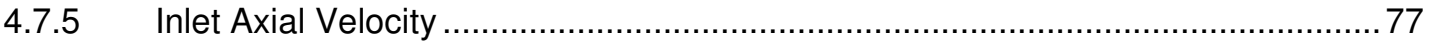




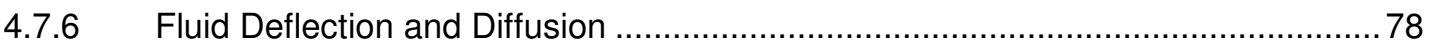

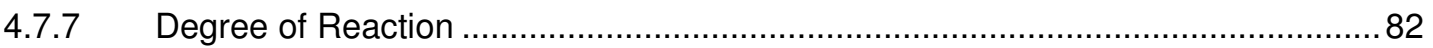

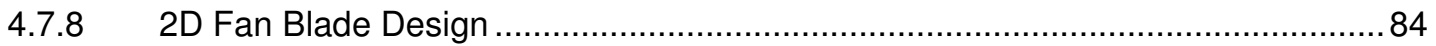

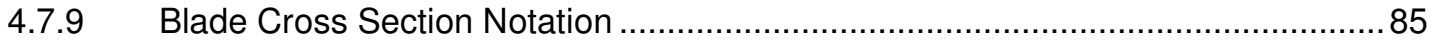

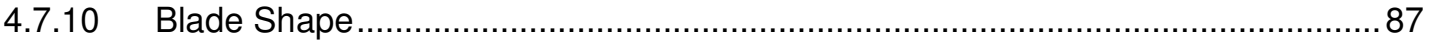

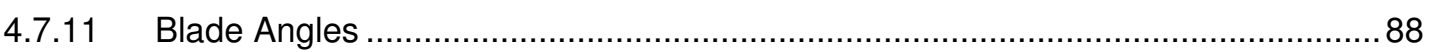

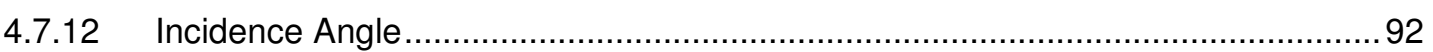

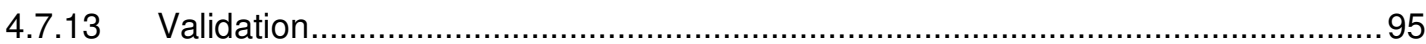

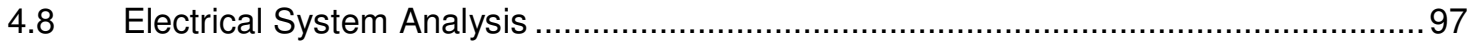

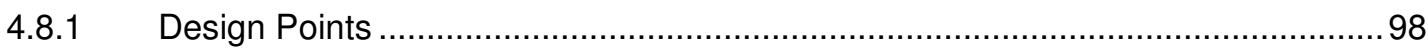

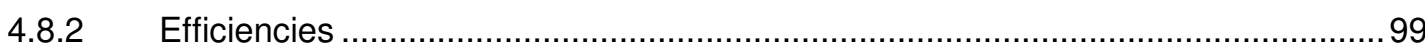

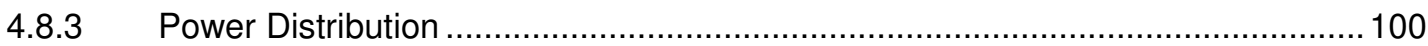

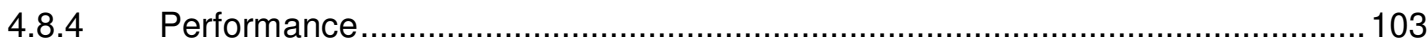

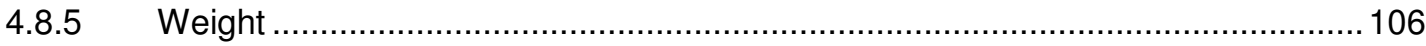

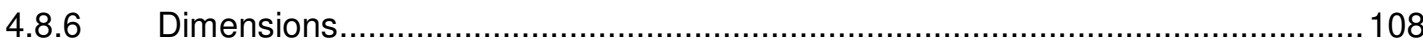

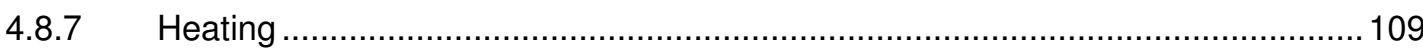

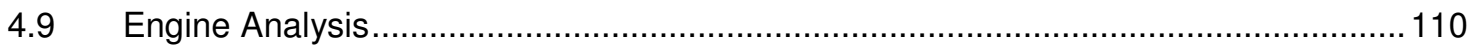

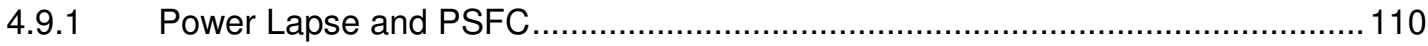

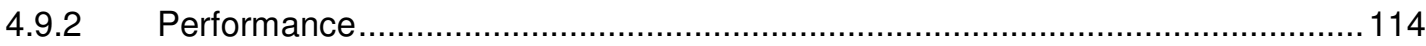

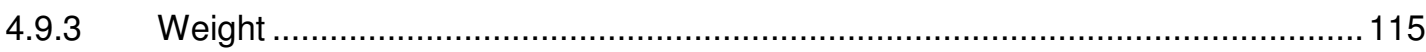

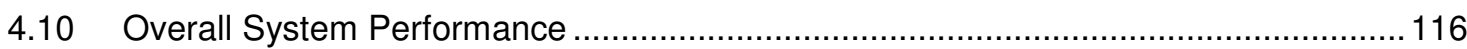

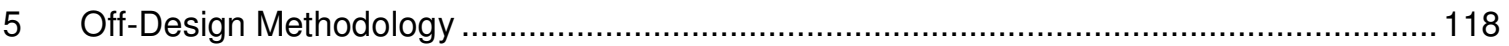

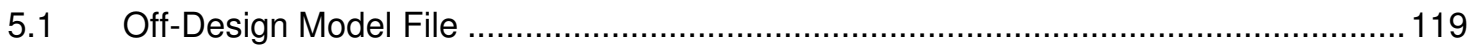

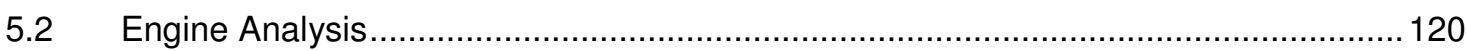

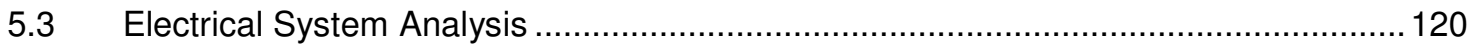

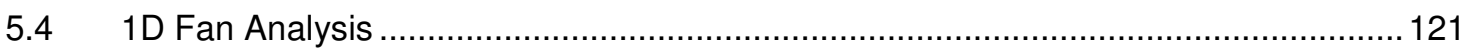

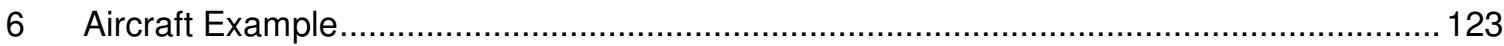

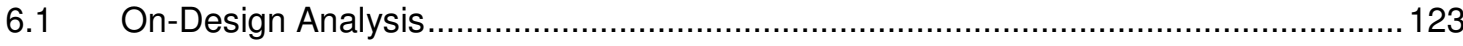

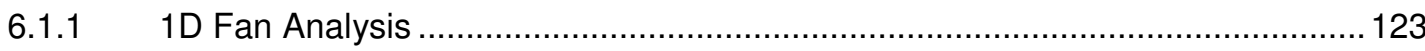

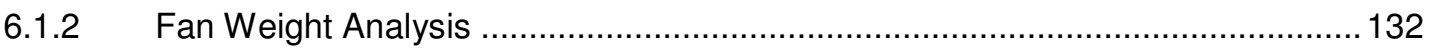

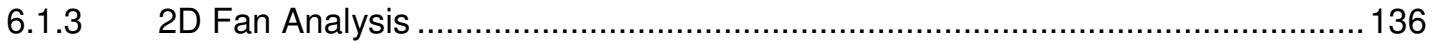

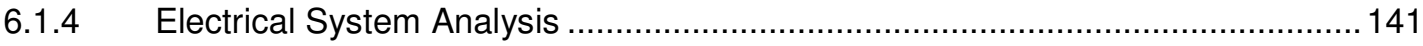

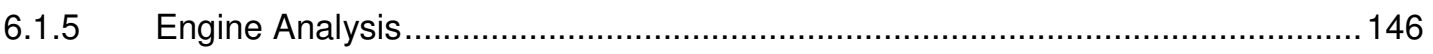

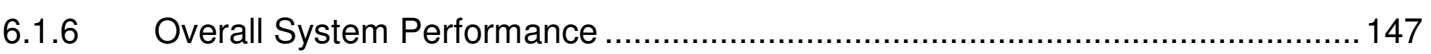

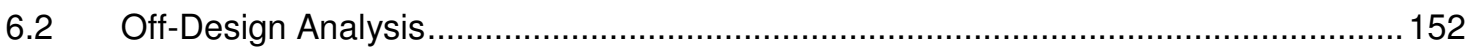

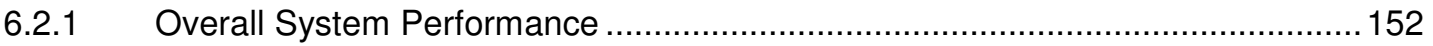

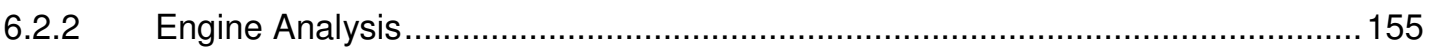

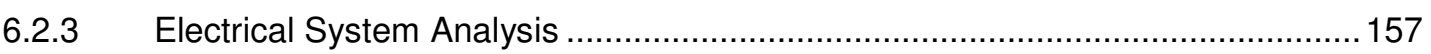




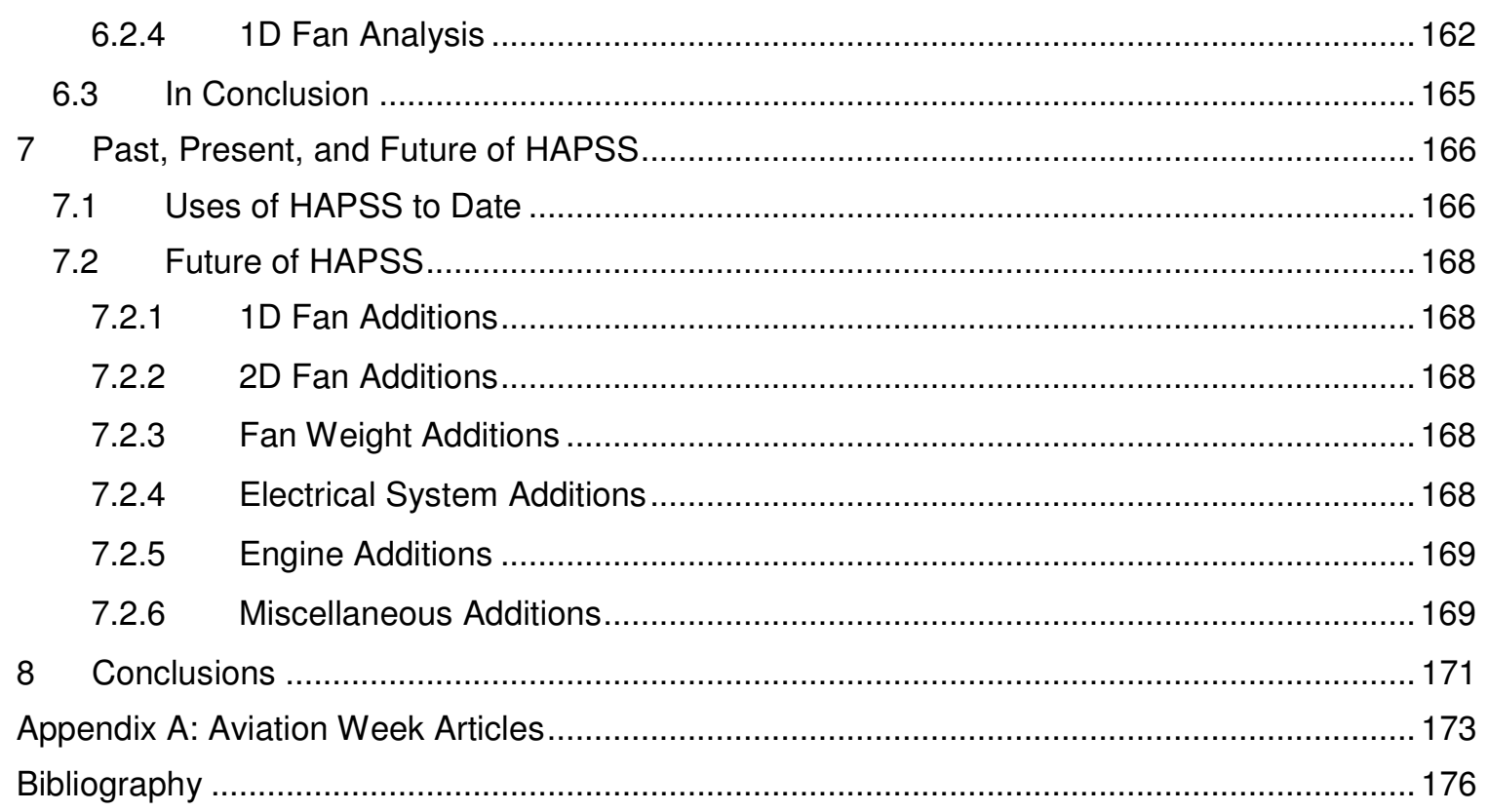




\section{List of Tables}

Table 1. Inputs and Outputs of the Thermodynamic Properties Function. ................................... 35

Table 2. Inputs and Outputs of the Thermodynamic Process Functions........................................ 38

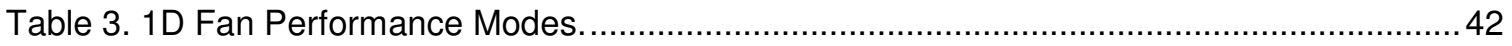

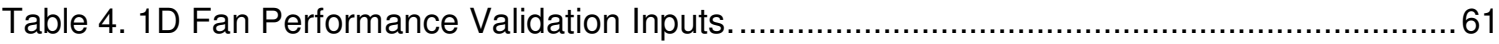

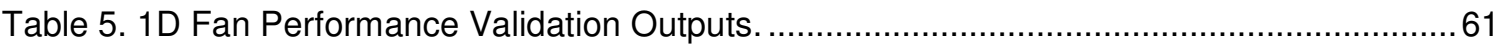

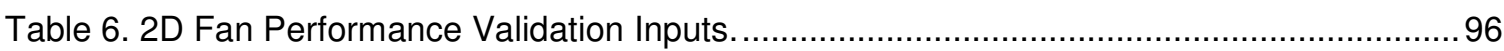

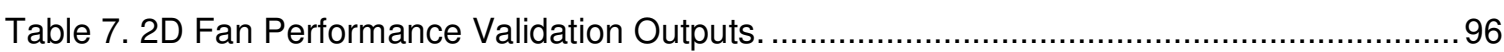

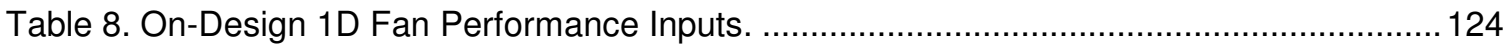

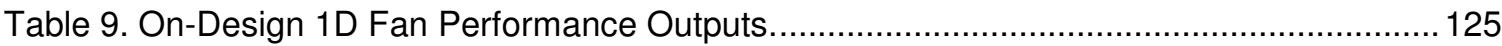

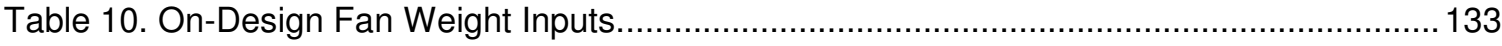

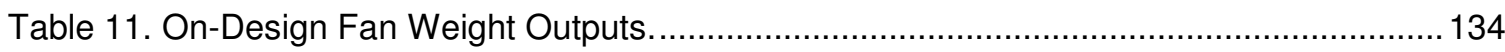

Table 12. On-Design 2D Fan Performance Outputs................................................................ 136

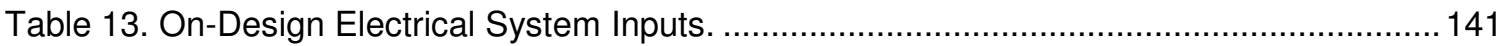

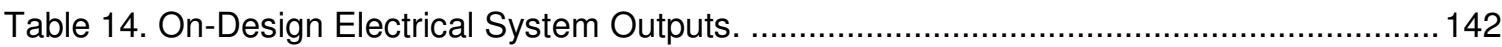

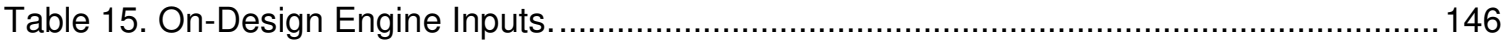

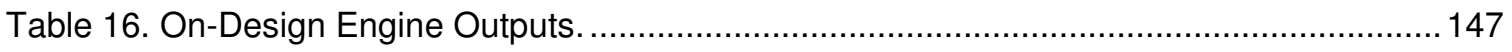

Table 17. On-Design Overall System Performance.............................................................. 147 


\section{List of Figures}

Figure 1. Typical Automotive Parallel Hybrid Configuration. ...................................................... 4

Figure 2. Typical Automotive Series Hybrid Configuration. ....................................................... 4

Figure 3. NASA N3-X Hybrid Propulsion Hybrid Wing Body Concept. .......................................... 7

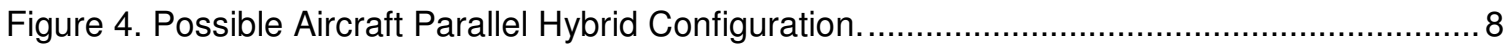

Figure 5. Possible Aircraft Series Hybrid Configuration......................................................... 9

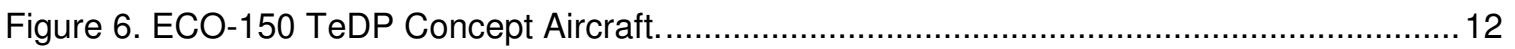

Figure 7. Illustration of a Two-spool Turbofan Engine. …...................................................... 13

Figure 8. Two-Spool CFM56-7B (left) and a Three-Spool Trent 1000 Turbofan (right)................ 14

Figure 9. Honeywell TPE331-10 Turboprop Performance Map................................................. 16

Figure 10. Generic Pure Series Hybrid System Diagram for HAPSS........................................25

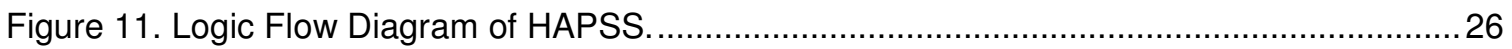

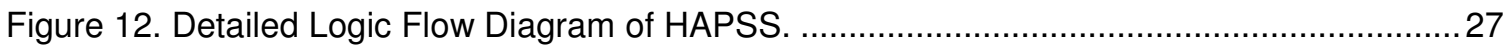

Figure 13. Detailed On-Design Logic Flow Diagram of HAPSS. ............................................. 31

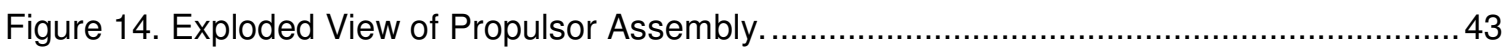

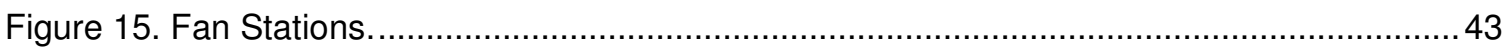

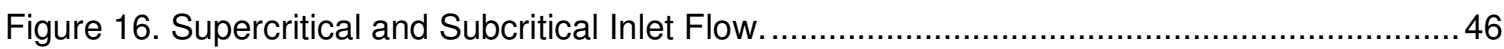

Figure 17. Example of Inlet Pressure Recovery vs. Flight Mach Number..................................47

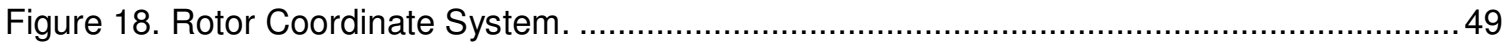

Figure 19. Example of Fan Power and Diameter vs. Fan Pressure Ratio..................................58

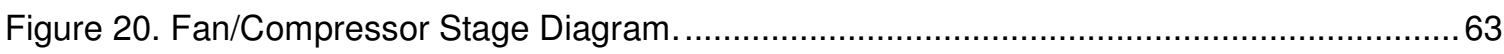

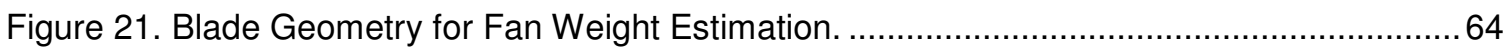

Figure 22. Relative Disc Thickness vs. Relative Disc Loading..............................................6 66

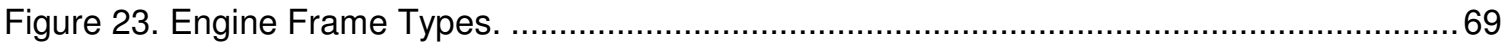

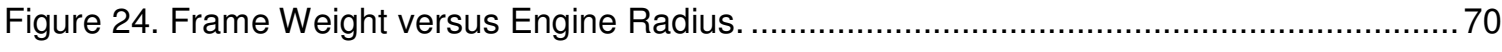

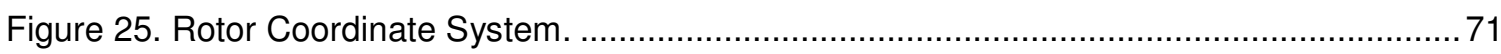

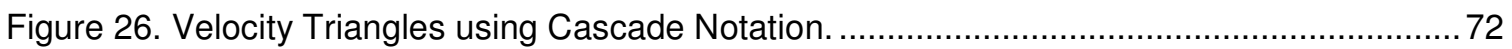

Figure 27. Relative Inlet Mach Number vs. Tip Mach Number and Air Inlet Angle. ...................... 74

Figure 28. Relative Mach Number vs. Blade Speed and Axial Velocity at Rotor Inlet. ................. 77

Figure 29. de Haller Number vs. Tip Mach Number and Air Inlet Angle ................................... 78

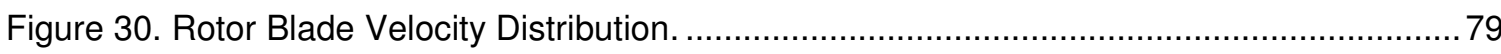

Figure 31. Rotor/Stator Solidity for Varying Air Deflection and Outlet Angle................................ 80

Figure 32. Total Pressure Loss Coefficient vs. NACA Diffusion Factor....................................... 81

Figure 33. Measure of Suction Loss vs. NACA Diffusion Factor. ................................................ 81

Figure 34. NACA Diffusion Factor vs. Tip Mach Number and Air Inlet Angle ..............................82

Figure 35. Degree of Reaction vs. Tip Mach Number and Air Inlet Angle..................................... 83 
Figure 36. NACA Diffusion Factor with 50\% Reaction Line. .84

Figure 37. General Electric GEnx (left) and GE90 (right) Fan Blades....................................... 85

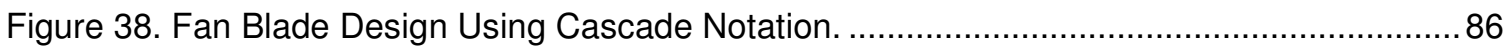

Figure 39. Example Double-Circular-Arc Blade Cross Section. ............................................... 87

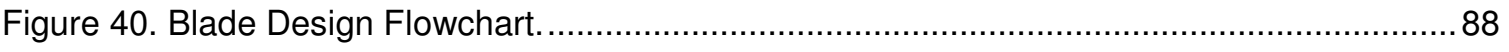

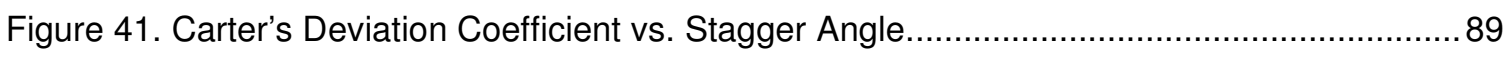

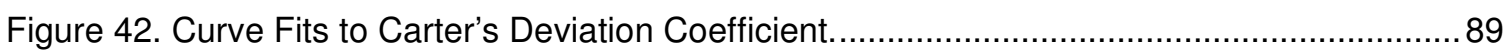

Figure 43. Required Fluid Deflection vs. Tip Mach Number and Air Inlet Angle. ........................90

Figure 44. Blade Camber Angle vs. Tip Mach Number and Air Inlet Angle.................................91

Figure 45.Blade Stagger Angle vs. Tip Mach Number and Air Inlet Angle .................................91

Figure 46. Deviation Angle vs. Tip Mach Number and Air Inlet Angle....................................... 92

Figure 47. Blade Incidence, Drag, and Deflection vs. Camber Angle. .........................................93

Figure 48. Total Pressure Loss and Deflection Angle vs. Incidence Angle..................................94

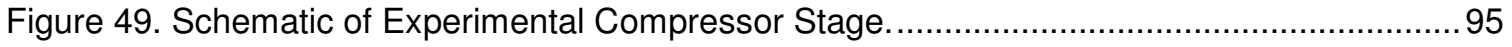

Figure 50. Generic Pure Series Hybrid System Diagram, Electrical System Highlighted. ............97

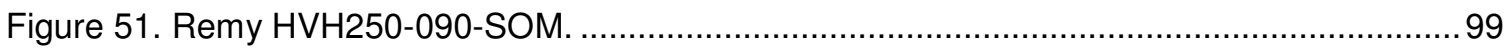

Figure 52. Remy HVH250-090-SOM Efficiency Map......................................................... 99

Figure 53. Electrical System Diagram, Battery Discharging..................................................101

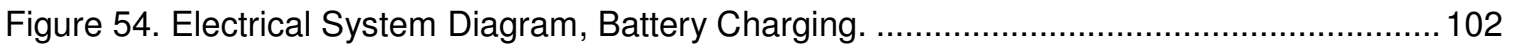

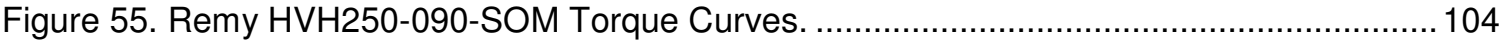

Figure 56. Remy HVH250-090-SOM Power Curves. .......................................................... 104

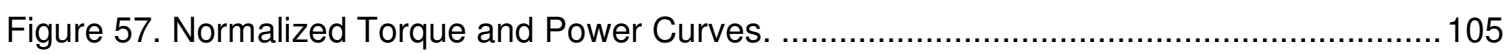

Figure 58. Motor/Generator Weight versus Maximum Peak Output Power. .............................. 107

Figure 59. Motor/Generator Length versus Output Torque and Diameter for $650 \mathrm{ft}-\mathrm{lb}_{\mathrm{ft}}{ }^{3} \ldots \ldots \ldots . .109$

Figure 60. Honeywell TPE331-10 Turboprop Performance Map. ............................................111

Figure 61. TPE331-10 PSFC and Power Lapse versus Mach Number at Full Power. ...............112

Figure 62. TPE331-10 PSFC and Power Lapse versus Power Setting at Zero Flight Speed.....113

Figure 63. Turbine Engine SFC at Maximum Power versus Maximum Power...........................114

Figure 64. Aircraft Turboshaft Weight versus Maximum Power. ............................................116

Figure 65. Detailed Off-Design Logic Flow Diagram of HAPSS. .......................................... 118

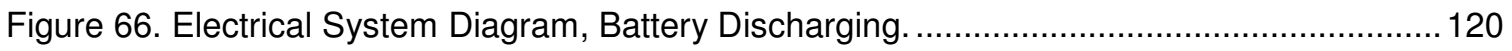

Figure 67. Fan Power versus Fan Pressure Ratio and Inlet Pressure Recovery....................... 126

Figure 68. Fan Diameter versus Fan Pressure Ratio and Inlet Pressure Recovery.................... 127

Figure 69. Fan Mass Flow Rate versus Fan Pressure Ratio and Inlet Pressure Recovery. ....... 127

Figure 70. Fan Speed versus Fan Pressure Ratio and Inlet Pressure Recovery........................ 128

Figure 71. Fan Torque versus Fan Pressure Ratio and Inlet Pressure Recovery......................128

Figure 72. Fan Propulsive Efficiency versus Fan Pressure Ratio and Inlet Pressure Recovery. 129 
Figure 73. Fan Diameter versus Number of Fans and Fan Pressure Ratio. 130

Figure 74. Fan Power versus Number of Fans and Fan Pressure Ratio. 131

Figure 75. Fan Speed versus Number of Fans and Fan Pressure Ratio. 131

Figure 76. Fan Torque versus Number of Fans and Fan Pressure Ratio. .................................132

Figure 77. Fan Weight versus Fan Pressure Ratio and Inlet Pressure Recovery...................... 135

Figure 78. Fan Weight versus Number of Fans and Fan Pressure Ratio.................................. 135

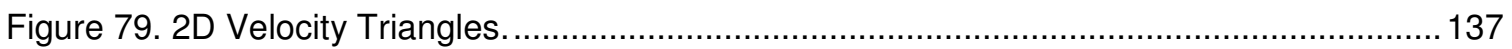

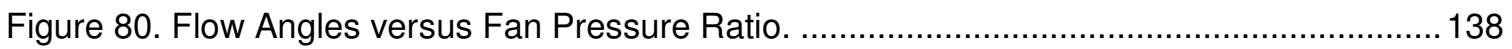

Figure 81. Blade Angles versus Fan Pressure Ratio......................................................... 139

Figure 82. Diffusion Factor and Degree of Reaction versus Fan Pressure Ratio........................ 140

Figure 83. Motor Weight versus Fan Pressure Ratio and Inlet Pressure Recovery.................... 144

Figure 84. Generator Weight versus Fan Pressure Ratio and Inlet Pressure Recovery............. 144

Figure 85. Motor Length versus Fan Pressure Ratio and Inlet Pressure Recovery................... 145

Figure 86. Generator Length versus Fan Pressure Ratio and Inlet Pressure Recovery.............145

Figure 87. TSFC versus Fan Pressure Ratio and Inlet Pressure Recovery............................. 148

Figure 88. Propulsive Efficiency versus Fan Pressure Ratio and Inlet Pressure Recovery. ....... 149

Figure 89. Thermal Efficiency versus Fan Pressure Ratio and Inlet Pressure Recovery............149

Figure 90. Overall Efficiency versus Fan Pressure Ratio and Inlet Pressure Recovery. ............150

Figure 91. Bypass Ratio versus Fan Pressure Ratio and Inlet Pressure Recovery................... 150

Figure 92. Total Weight versus Fan Pressure Ratio and Inlet Pressure Recovery....................151

Figure 93. Total Thrust versus Flight Mach Number and Altitude. ........................................... 153

Figure 94. TSFC versus Flight Mach Number and Altitude. ................................................... 153

Figure 95. Propulsive Efficiency versus Flight Mach Number and Altitude. .............................. 154

Figure 96. System Thermal Efficiency versus Flight Mach Number and Altitude....................... 154

Figure 97. Overall Efficiency versus Flight Mach Number and Altitude..................................... 155

Figure 98. Total Engine Power versus Flight Mach Number and Altitude................................. 156

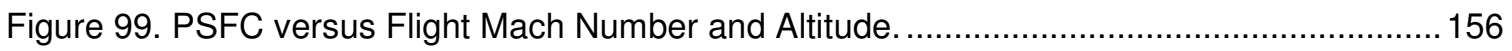

Figure 100. Motor Efficiency versus Flight Mach Number and Altitude...................................157

Figure 101. Generator Efficiency versus Flight Mach Number and Altitude.............................. 157

Figure 102. Electrical System Efficiency versus Flight Mach Number and Altitude. .................. 158

Figure 103. Motor Heating versus Flight Mach Number and Altitude...................................... 159

Figure 104. Generator Heating versus Flight Mach Number and Altitude................................. 159

Figure 105. Controller Heating versus Flight Mach Number and Altitude. .................................. 160

Figure 106. Motor Percent of Max Peak Power versus Flight Mach Number and Altitude. ........ 160

Figure 107. Motor Percent of Max Cont Power versus Flight Mach Number and Altitude.......... 161

Figure 108. Generator Percent of Max Peak Power versus Flight Mach Number and Altitude. . 161

Figure 109. Generator Percent of Max Cont Power versus Flight Mach Number and Altitude... 162 
Figure 110. Fan Pressure Ratio versus Flight Mach Number and Altitude. .............................. 163

Figure 111. Fan Speed versus Flight Mach Number and Altitude............................................ 163

Figure 112. Fan Tip Mach Number versus Flight Mach Number and Altitude............................ 164

Figure 113. Fan Face Mach Number versus Flight Mach Number and Altitude......................... 164

Figure 114. Fan Mass Flow Rate versus Flight Mach Number and Altitude. ............................ 165 


\section{Nomenclature}

\section{English Symbols}

\begin{tabular}{|c|c|}
\hline Symbol & Definition \\
\hline$A$ & Area \\
\hline$A R$ & Blade aspect ratio \\
\hline$B P R$ & Bypass ratio \\
\hline C & Absolute velocity \\
\hline$D$ & Diameter \\
\hline$D F$ & Diffusion factor \\
\hline$D H N$ & de Haller number \\
\hline$F$ & Thrust \\
\hline FoS & Factor of safety \\
\hline$H$ & Total enthalpy \\
\hline I & Conversion factor, $550(\mathrm{ft}-\mathrm{lbf} / \mathrm{s}) / \mathrm{hp}$ \\
\hline J & Conversion factor, $778.16 \mathrm{ft}-\mathrm{lb}_{\mathrm{f}} / \mathrm{BTU}$ \\
\hline$K_{f}$ & Fan flow coefficient \\
\hline$K_{p}$ & Fan power coefficient \\
\hline$K_{v o l}$ & Blade volume factor \\
\hline L & Length \\
\hline LHV & Lower heating value \\
\hline M & Mach number \\
\hline$N$ & Rotational speed \\
\hline$P$ & Total pressure \\
\hline$P L$ & Power lapse \\
\hline$P R$ & Pressure recovery or pressure loss \\
\hline PSFC & Power specific fuel consumption \\
\hline Pwr & Power \\
\hline$Q$ & Volumetric flow rate \\
\hline $\bar{R}$ & Universal gas constant, 1545 ft-l列/(lbmol- $\left.{ }^{\circ} \mathrm{R}\right)$ \\
\hline$T$ & Total temperature, or torque \\
\hline$T R$ & Blade taper ratio \\
\hline TSFC & Thrust specific fuel consumption \\
\hline$U$ & Blade tip velocity \\
\hline V & Velocity, or relative velocity \\
\hline V & Volume \\
\hline W & Weight \\
\hline$Z$ & Altitude \\
\hline$a$ & Static acoustic speed, or blade chord location of maximum camber \\
\hline$c$ & Blade chord length \\
\hline$c_{p}$ & Specific heat at constant pressure \\
\hline$f$ & Fuel-to-air ratio \\
\hline far & Fuel-to-air ratio \\
\hline$g_{c}$ & Gravitational constant, $32.174 \mathrm{lb}_{\mathrm{m}}-\mathrm{ft} /\left(\mathrm{lb}_{\mathrm{f}}-\mathrm{s}^{2}\right)$ \\
\hline$h$ & Static enthalpy or blade height \\
\hline htr & Hub-to-tip ratio \\
\hline$i$ & Incidence angle \\
\hline$m$ & Carter's deviation coefficient \\
\hline$\dot{m}$ & Air mass flow rate \\
\hline$n$ & Number of \\
\hline$p$ & Static pressure \\
\hline$t$ & Static temperature or thickness \\
\hline$q$ & Heat transfer rate \\
\hline
\end{tabular}




$\begin{array}{ll}r & \text { Radius } \\ s & \text { Static entropy, or blade pitch } \\ s / c & \text { Pitch chord ratio } \\ \dot{w} & \text { Fuel flow rate } \\ w f f & \text { Weight flow function }\end{array}$

\section{Greek Symbols}

$\begin{array}{ll}\text { Symbol } & \text { Definition } \\ \alpha & \text { Absolute air flow angle } \\ \beta & \text { Relative air flow angle } \\ \Delta & \text { Change in } \\ \delta & \text { Pressure ratio, or blade deviation angle } \\ \varepsilon & \text { Fluid deflection angle } \\ \eta & \text { Efficiency } \\ \gamma & \text { Ratio of specific heats } \\ \Lambda & \text { Degree of reaction } \\ \Lambda_{N} & \text { Motor speed fraction } \\ \Lambda_{T} & \text { Motor torque fraction } \\ \mu & \text { Dynamic viscosity } \\ \omega & \text { Angular velocity } \\ \pi & \text { Pressure ratio } \\ \phi & \text { Nozzle velocity coefficient } \\ \rho & \text { Density } \\ \sigma & \text { Density ratio, solidity, or stress } \\ \tau & \text { Shear stress } \\ \theta & \text { Temperature ratio, or blade camber angle } \\ \xi & \text { Blade stagger angle }\end{array}$

\section{Subscripts}

$\begin{array}{ll}\text { Symbol } & \text { Definition } \\ a & \text { Axial } \\ a m b & \text { Ambient } \\ b & \text { Blade } \\ \text { batt } & \text { Electrical battery } \\ c & \text { Corrected } \\ \text { case } & \text { Fan casing } \\ \text { cont } & \text { Electrical controller } \\ \text { disc } & \text { Rotor disc } \\ e & \text { Exit } \\ \text { eng } & \text { Engine } \\ f & \text { Fan } \\ \text { fuel } & \text { Engine fuel } \\ \text { gen } & \text { Electrical generator } \\ h & \text { Hub } \\ h p & \text { Horsepower } \\ h w & \text { Fan hardware } \\ \text { in } & \text { Input to } \\ \text { motor } & \text { Electrical motor } \\ n & \text { Nozzle } \\ o & \text { Overall }\end{array}$




$\begin{array}{ll}\text { out } & \text { Output from } \\ p & \text { Polytropic, or propulsive } \\ r & \text { Fan blade root } \\ \text { rel } & \text { Relative } \\ \text { rotor } & \text { Fan rotor } \\ s & \text { Isentropic } \\ \text { shaft } & \text { Fan shaft } \\ \text { sls } & \text { Sea level static } \\ \text { stator } & \text { Fan stator } \\ \text { system } & \text { Electrical system } \\ t & \text { Fan blade tip } \\ \text { tip } & \text { Fan blade tip } \\ t h & \text { Thermal } \\ w & \text { Whirl } \\ 0 & \text { Sea level reference } \\ 2 & \text { Rotor entrance } \\ 25 & \text { Rotor exit / stator entrance } \\ 3 & \text { Stator exit / nozzle entrance } \\ 6 & \text { Nozzle exit }\end{array}$




\section{Introduction}

\subsection{What is a Hybrid Propulsion System?}

Merriam-Webster defines "hybrid" as "an offspring of two animals or plants of different races, breeds, varieties, species, or genera." This thesis however does not discuss the crossbreeding of animals or plants. In recent years the term "hybrid" has been used heavily by the automotive market to describe the latest vehicle architecture. The motive force behind a traditional automobile (species A) is a single internal combustion engine driving the wheels through multiple gearboxes. An all-electric vehicle (species B) is powered by an electric motor and a battery pack. A hybrid vehicle on the other hand combines the internal combustion engine from species A with an electric motor from species B. The most well-known hybrid vehicle today is the Toyota Prius. The 2011 model features a $98 \mathrm{hp} 1.8 \mathrm{~L}$ 4-cylinder internal combustion engine, an $80 \mathrm{hp}$ electric motor, and a nickel-metal hydride (Ni-MH) battery pack. With its hybrid propulsion system the Toyota Prius can achieve a combined gas mileage of $50 \mathrm{mpg}$ with a range of nearly 600 miles with just 11.9 gal of fuel. Section 1 will continue to discuss generic and automobile hybrid systems as a fundamental understanding of this technology is necessary before considering hybrid aircraft systems.

\subsection{Hybrid System Components}

\subsubsection{Fuel Tank}

In a hybrid vehicle system, there are two sources of energy; the fuel in the fuel tank and the energy stored in the battery. For automobiles the fuel is typically gasoline or diesel, but can also be other kerosene-based fuels such at Jet-A, hydrogen, or even coal, anything that is combusted to release its stored chemical energy. Gasoline contains about 20,000 BTU/lb of energy while diesel contains about 19,300 BTU/lb. Hydrogen contains much more energy at 
$61,000 \mathrm{BTU} / \mathrm{lb}$. This value is commonly referred to as the lower heating value or heat of combustion.

\subsubsection{Battery Pack}

The battery pack of a hybrid vehicle is the second source of energy. The energy is released via a chemical reaction inside the battery when energy is demanded by the electrical system. While the Toyota Prius uses Ni-MH batteries, today's higher performance hybrid and allelectric vehicles are utilizing Lithium-Ion (Li-lon) batteries. This type of battery is commonly found in laptops and cordless power tools, and boasts a specific energy of around $250 \mathrm{Wh} / \mathrm{kg}$ for the best performing cells. This value equates to $387 \mathrm{BTU} / \mathrm{lb}$, roughly $2 \%$ of the energy contained in gasoline. Battery technology has accelerated in the past decade and new chemistries are being developed including Lithium-Sulfur, Lithium-Air, and Magnesium-Air. A Lithium-Air battery with the use of oxygen has a theoretical specific energy of 8060 BTU/lb, roughly $40 \%$ the energy content of gasoline.

\subsubsection{Internal Combustion Engine}

The internal combustion engine converts the chemical energy of the fuel into mechanical energy. Between a hybrid vehicle and a traditional automobile, there is little difference in the design of the engine. Because a hybrid vehicle has two sources of energy, its engine can generally be smaller than most traditional vehicles, and thus consume less fuel. Modern gasoline engines have an average efficiency of about $20 \%$, meaning that approximately $80 \%$ of the energy stored in the gasoline is expended as heat, noise, or is used to overcome friction losses in the engine. The engine's efficiency varies greatly with throttle/power output. High-efficiency diesel engine manufactures have claimed efficiencies up to $40 \%$.

\subsubsection{Electric Motor}

In a hybrid vehicle, the electric motor drives the wheels directly or through a mechanical gearbox. The motor is supplied with electricity from the battery which is first fed through a controller. The electric motor then converts the electrical energy into mechanical energy. Electric motors are notorious for high torque and high efficiency, greater than $90 \%$ in most cases. Electric 
motors also tend to have a flat efficiency curve, meaning their efficiency is very high for a wide range of power outputs. Typical input voltages for hybrid car electric motors range from 200-600 volts and today's hybrid vehicles typically utilize alternating current $(A C)$ motors and generators.

\subsubsection{Electric Generator}

An electric generator is essentially the mirror image of an electric motor; instead of outputting mechanical power, the generator is rotated via an external mechanical power source (an internal combustion engine in the hybrid vehicle case) and produces electrical power. This power is then fed back through the controller and recharges the battery pack. Generators have similar efficiencies and voltage requirements as motors, and in reality many motors can be used in reverse as generators while delivering nearly identical performance. This unique characteristic of motors/generators is important as explained later.

\subsubsection{Controller}

In a hybrid vehicle the controller is the "brains" of the hybrid system. The controller manages the distribution of power between the generator, motor, and battery. The controller determines how much power to deliver to the motor based on the driver's power demand (gas pedal position) and the vehicle's current state (speed, component temperature, battery state-ofcharge, etc). The controller also determines when to start the internal combustion engine to charge the battery or to provide supplemental power to the system.

\subsection{Parallel vs. Series Systems}

There are two traditional types of hybrid configurations; parallel and series. A parallel configuration utilizes an internal combustion engine that drives the wheels directly through mechanical connections (shafts/gearboxes). In addition, there is an electrical system that provides supplemental power to the wheels via an electric motor, batteries, etc. A series hybrid configuration uses an internal combustion engine that solely drives a generator producing electrical power. This power is then distributed to the battery and the electric motor which drives 
the wheels. Figure 1 and Figure 2 illustrate typical automotive parallel and series hybrid configurations, respectively.

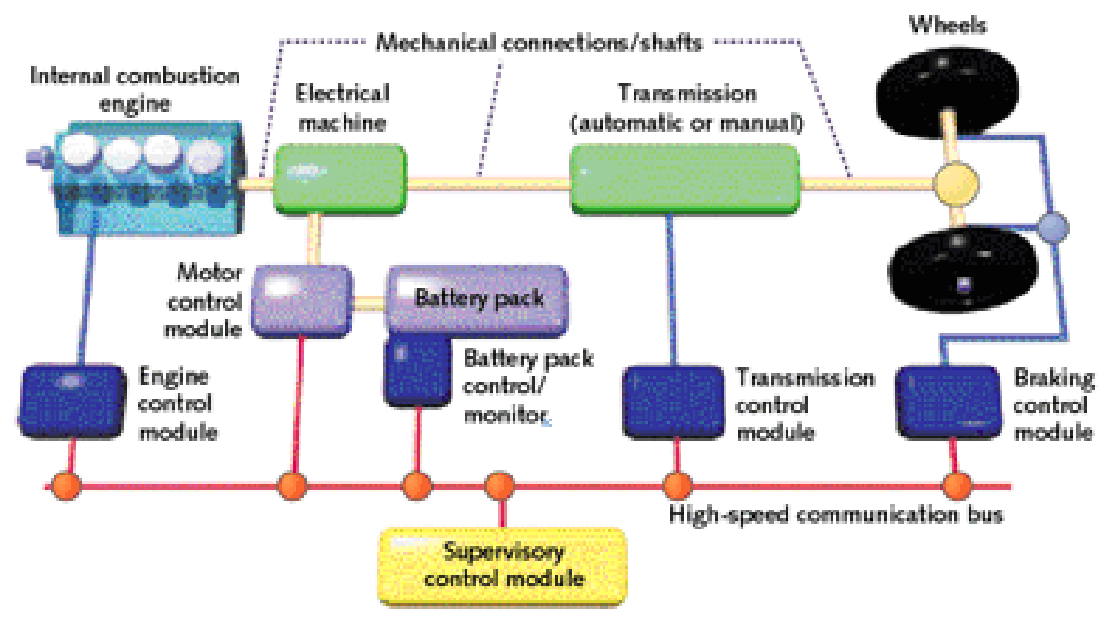

Figure 1. Typical Automotive Parallel Hybrid Configuration'.

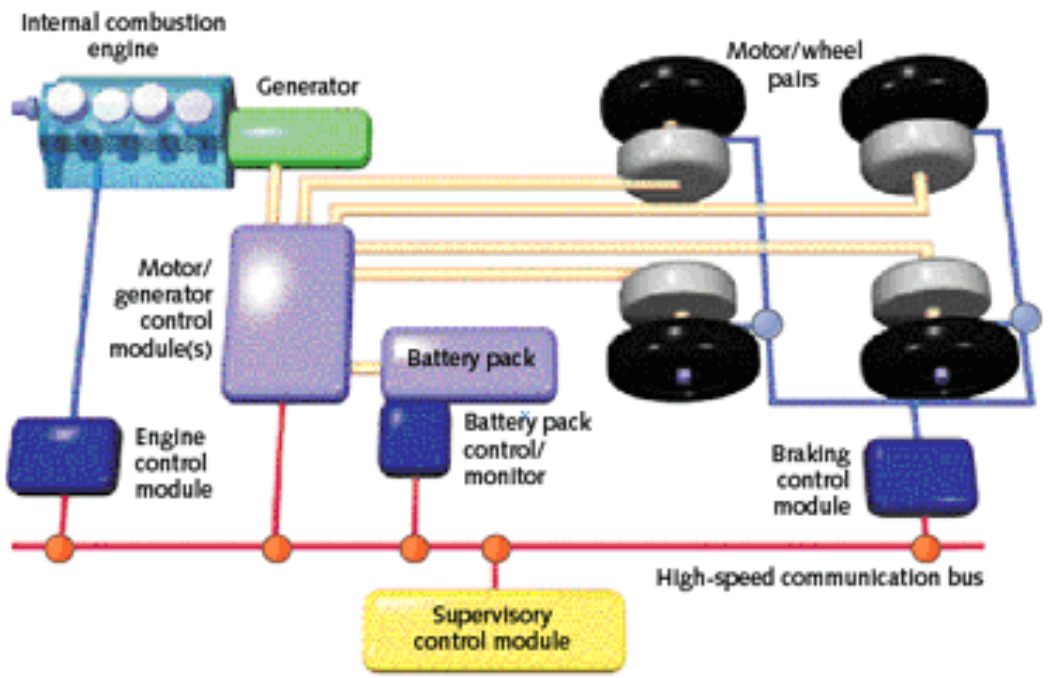

Figure 2. Typical Automotive Series Hybrid Configuration'.

These two figures feature all of the components described in section 1.2 in addition to other essential hybrid system components. The Toyota Prius however is commonly referred to as a power-split hybrid or parallel-series hybrid since it incorporates features from both pure parallel and pure series configurations. The internal combustion engine in a Prius is allowed to power the wheels through either a mechanical connection (parallel) or an electrical connection (series) through the use of a power-split device. Of course there are countless different types of hybrid 
configurations each with unique benefits and drawbacks. To provide a more meaningful discussion on the advantages and disadvantages of hybrid systems, the remainder of this report will focus on aircraft specific applications of hybrid system technology. 


\section{Aircraft Hybrid Propulsion}

\subsection{Introduction}

The hybrid system concept can be applied to an aircraft in much the same fashion as an automobile. In an automobile, the component that directly moves the vehicle is the wheel/tire, which utilizes friction to apply a force to the road, accelerating the vehicle in the opposite direction. In an aircraft however, the vehicle is accelerated by means of propulsive fans, or propulsors, which ingest air at the front, accelerate it by doing work on or applying energy to the air, and then exhaust it out the back at a greater velocity. The amount of forward force that can be generated is a function of the mass of ingested air and the velocity that was added to the air. The means of propulsion, tires versus fans, is the primary difference between automotive and aircraft hybrid systems.

In a hybrid automobile, the internal combustion engine consumes fuel to produce electrical power via a generator attached directly to the engine. In an aircraft application, this concept remains unchanged. In an automobile, the internal combustion engine is typically an Otto cycle engine consuming gasoline or diesel. An aircraft however may use a variety of engine types from an Otto cycle to a more conventional Brayton (turbine-based) cycle engine, depending on the application. Otto cycle engines generally offer excellent fuel efficiency and ease of integration but with poor performance at altitude and generally low power density (output power per unit mass). Brayton cycle engines however offer very high power densities and perform better at altitude but at the cost of reduced fuel efficiency added vehicle integration difficulties. With any engine architecture the engine drives a generator that produces electrical power. The design of the generator depends highly on the engine cycle due to the wide range of engine possible output shaft speeds.

On a hybrid aircraft, the electrical power produced by the generator is transmitted to the controller, similar to a hybrid automobile. The controller distributes the power to the propulsor, to the battery pack, or to both, depending on the inputs from the pilot and the state of the aircraft. 
The battery pack for a hybrid aircraft would be designed similarly to that of an automobile with the size depending on the energy, power, and voltage requirements. The core battery chemistry would likely be identical between automobiles and aircraft. While the battery sector dedicated to aircraft remains small for vehicles larger than radio control models, technology for hybrid automobile batteries is developing rapidly and can be applied directly to large hybrid aircraft. Figure 3 shows a hybrid wing body (HWB) concept from NASA named the N3-X. This concept vehicle was designed utilizing a specific hybrid propulsion system configuration called turboelectric distributed propulsion (TeDP). This type of system is further discussed in section 2.3 and is the basis of this project.

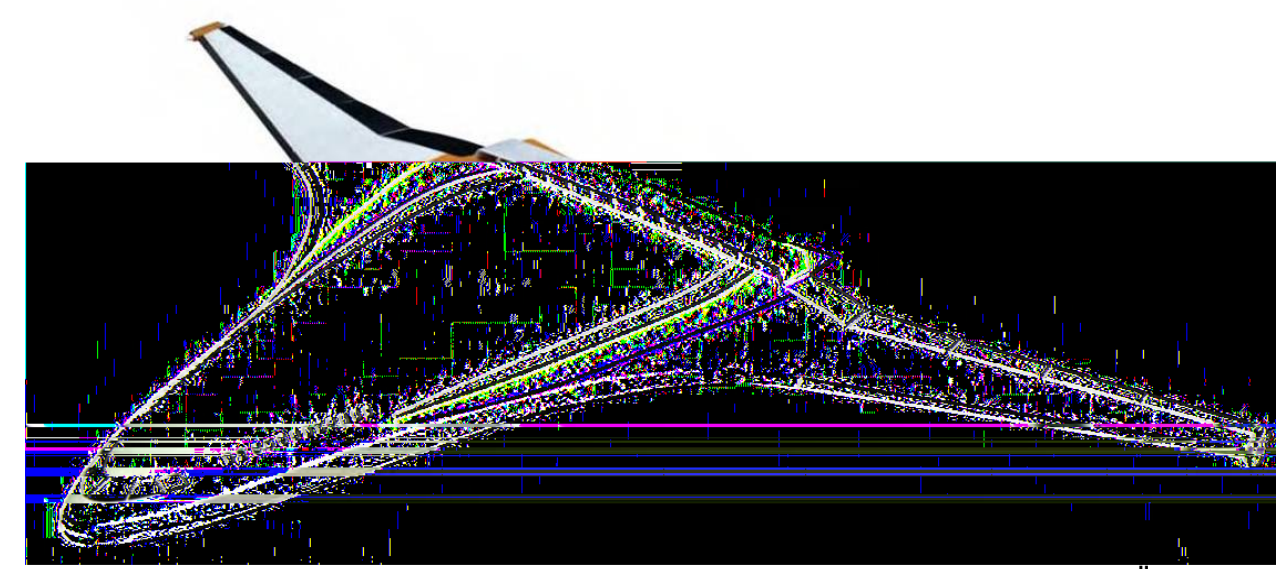

Figure 3. NASA N3-X Hybrid Propulsion Hybrid Wing Body Concept".

\subsection{Series vs. Parallel Systems for Aircraft}

A final key difference between automotive and aircraft hybrid systems is that an aircraft hybrid system is most likely assume a series configuration like that illustrated in Figure 2. A parallel hybrid system utilizes an internal combustion engine driving the propulsive component directly with a separate parallel electrical system providing supplemental power. Applied to an aircraft, parallel hybrid architecture would yield a system with propulsive propellers or fans mechanically attached to the engine's output shaft either directly or via a gearbox. These engines would also operate generators providing power to electric motors that are too attached to the shafts of the fans or propellers. The electric motors could also be used to drive separate propellers/fans to provide supplemental thrust. Unsurprisingly, this type of system would be 
mechanically complex, heavy, and burdened with inefficiencies. Figure 4, an adaptation of Figure 1 for an aircraft application, shows a system view of a potential aircraft parallel hybrid configuration.

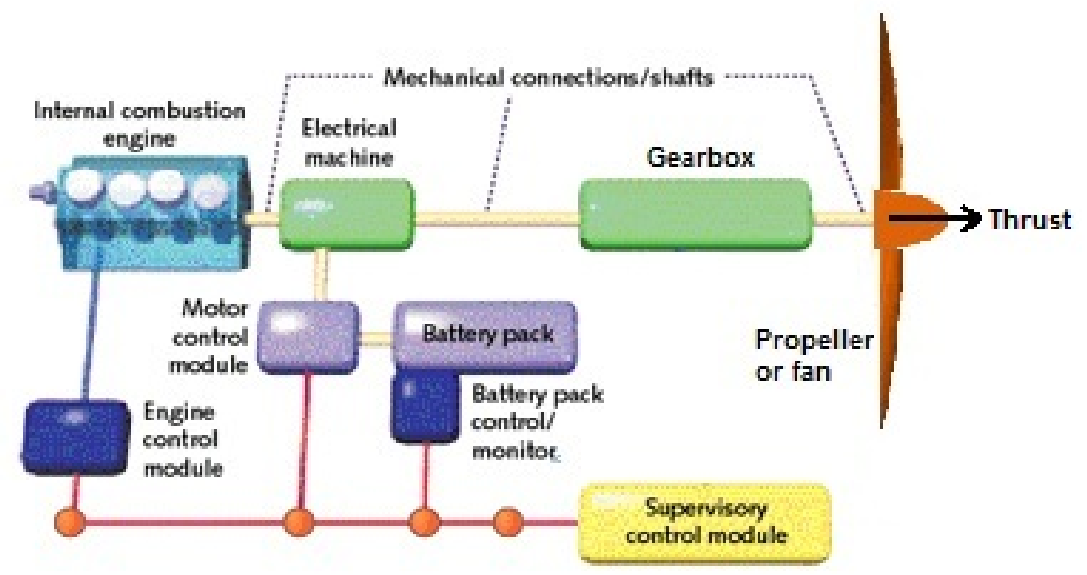

Figure 4. Possible Aircraft Parallel Hybrid Configuration.

In this figure, the wheels, the propulsive component of an automobile hybrid system, have been replaced with a propeller/fan element which provides propulsive force for an aircraft. The braking module has been removed since aircraft do not use automotive style braking systems in flight. And finally, the transmission, found in the automobile hybrid system, has been renamed a gearbox for the aircraft application. Automotive style transmissions with selectable gears are rarely seen on aircraft on account of their additional mass, complexity, and difficulty withstanding the power requirements of contemporary fans and propellers.

Series hybrid architecture decouples the internal combustion engine from the propulsive component, permitting much more freedom in the design of the system. Figure 5, a revised version of Figure 2, shows a system view of a possible series hybrid configuration for an aircraft. 


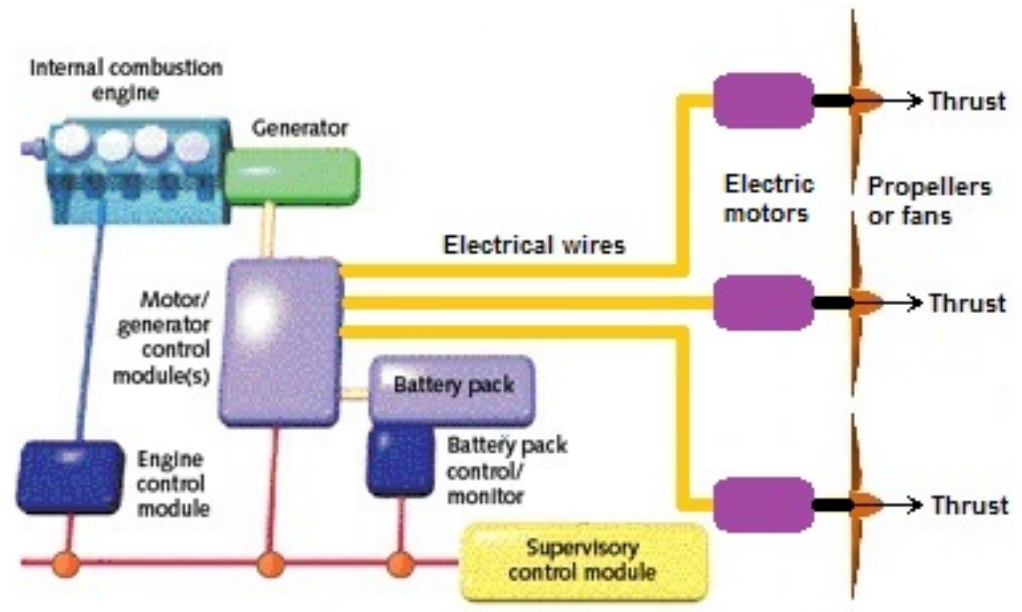

Figure 5. Possible Aircraft Series Hybrid Configuration.

As with Figure 4, the wheels have been replaced with propeller/fan elements which provide the propulsive force to the aircraft. The primary difference between the series system in Figure 5 and the parallel system in Figure 4 is the lack of mechanical connections/shafts to the propellers and the gearbox. Power is transmitted from the controller to the propellers/fans via electric wires and motors. The only mechanical connections that exist are those between the engine and the generator, and between the electric motor and the propeller/fan. In an optimal aircraft series configuration, these mechanical connections are likely very short direct-drive with virtually no mechanical losses. The flexibility in motor and generator design allow them to be designed to operate at nearly any speed desired, virtually eliminating the need for bulky, inefficient gearboxes.

Since electric wires can be routed in almost any configuration, and electric motors are generally compact, the propellers/fans can be mounted in nearly any feasible location on the aircraft. Furthermore, it is far simpler to split and distribute power via electric cabling than through mechanical shafts and gearboxes, making it possible to operate many propeller/fan elements using just one or a few power sources such as engines and batteries.

While countless hybrid architectures utilizing parallel and series characteristics could be devised for aircraft, the pure series hybrid is the most simple in terms of understanding, modeling, and implementation. A basic knowledge of how a pure series hybrid system can be applied to an aircraft and its potential benefits is necessary before contemplating more complex hybrid 
systems. Additionally, with the groundwork laid for a pure series aircraft hybrid, the modifications required to model other architectures are minimal.

This project focused exclusively on pure series hybrid propulsion configurations like that illustrated in Figure 5. A pure series hybrid propulsion system will be defined as a system without any mechanical connections linking the fuel consuming component (engine) to the thrust producing component (propeller/fan). Power is transferred between components via one mode only (mechanical, electrical, etc). Secondly, it was assumed that each engine was paired with a single electric generator, and that each electric motor was paired with a single propeller/fan. This type of configuration was simple enough to code, but is valuable enough to study.

\subsection{Advantages of Hybrid Aircraft Propulsion}

The benefits of a hybrid system depend highly on the configuration; series versus parallel or any combination thereof. As explained in section 2.2, this project deals with only pure series hybrid systems, the advantages of which will be discussed in this section. The benefits of this specific system type however are applicable to most other conceivable hybrid system architectures. It is important to acknowledge the advantages of hybrid propulsion in order to understand the desire for a program that can analyze these types of systems.

\subsubsection{Distributed Propulsion}

As the name suggests, distributed propulsion implies that the propulsive force applied to the aircraft is distributed about the aircraft instead of at a single or few points. A single-engine Cessna is not a distributed propulsion aircraft because the engine and propeller produce thrust at a single point. However, a Boeing 737 with two turbofan engines or a 747 with four turbofan engines both may be considered distributed propulsion vehicles by some, but in the spirit of the concept, they and similar aircraft should not. Instead, an improved definition could be useful; "Distributed propulsion in aircraft application is the spanwise distribution of the propulsive thrust stream such that overall vehicle benefits in terms of aerodynamic, propulsive, structural, and/or other efficiencies are mutually maximized to enhance the vehicle mission." ${ }^{\text {,iii }}$ While this definition is still somewhat vague, "spanwise distribution of the propulsive stream thrust" is the primary 
element of distributed propulsion. Nevertheless, it could still be considered a distributed propulsion system if the propulsors were placed elsewhere on the aircraft than the main wing, such as the fuselage or empennage.

Arguably one of the greatest advantages of pure series hybrid propulsion architecture is that it lends itself well to distributed propulsion. By utilizing electrical wiring instead of mechanical shafting to transmit power to the propulsive components, fans or propellers can be placed in almost any feasible location on the aircraft. Electrical cabling can be routed in nearly any imaginable configuration, through or around wing spars and ribs, around fuel tanks, and even through the wingbox and fuselage. Mechanical shafts can only be routed in straight sections utilizing gears or universal joints for bends. This can quickly lead to a mechanically complex, heavy, inefficient, unreliable, and noisy power transmission system, not to mention a maintenance nightmare. Attempting a true distributed propulsion system via mechanical connections would be a difficult undertaking.

Utilizing a series hybrid system, the thrust producing component does not need to be located on or near the power producing component like a typical turbofan engine, allowing for much more variability with propulsion integration. Furthermore, transmitting power via electrical wires eliminates the need for power splitting gearboxes. The electrical power generated by one engine/generator combination can be split in any desirable proportion to any number of propulsors with minimal losses. This flexibility is simply not feasible with a parallel hybrid or nonhybrid system, both of which require mechanical shafting.

One distributed propulsion configuration that is commonly researched is turboelectric distributed propulsion (TeDP). This propulsion concept utilizes a pure series hybrid propulsion system. Turboshaft engines coupled with generators produce the electrical energy, battery packs store the energy, and electric motors coupled with propulsive fans consume the energy and provide thrust. In this type of system, there are typically numerous small fans placed across the span of the main wing. Figure 6 shows the ECO-150, a 150 passenger TeDP aircraft designed by ESAero to explore future vehicle concepts for improved efficiency, emissions, and aerodynamics. This vehicle utilizes a total of 16 electric fans embedded inside the inboard section of the main 
wing. Just to the outside of these fans are two turboshaft engines and generators which provide the electrical power to the propulsors.

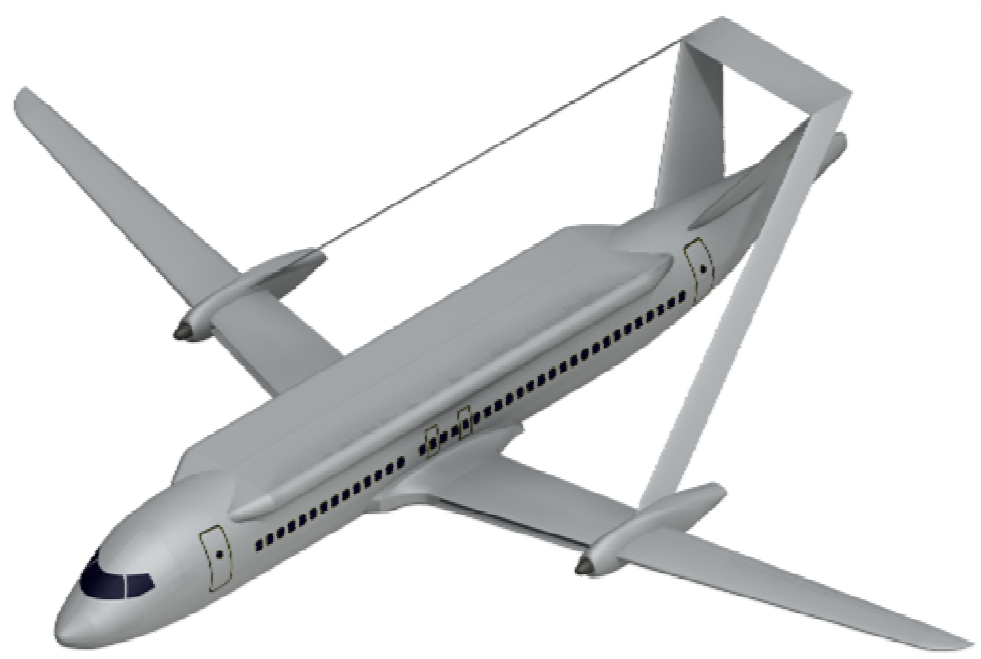

Figure 6. ECO-150 TeDP Concept Aircraft ${ }^{\mathrm{iv}}$.

\subsubsection{Decoupling}

Another noteworthy advantage of aircraft hybrid propulsion systems is the ability to decouple the power producing device from the thrust producing device. In a traditional turbofan engine there are two spools, low-pressure and high-pressure. Each spool consists of a compressor and a turbine, connected together by a central shaft. The shafts of the two spools are coaxial, meaning the shaft of the low-pressure spool rotates inside the shaft of the high-pressure spool. The low-pressure spool also rotates the fan which is the primary producer of thrust in a turbofan. Figure 7 shows a simple illustration of a two-spool turbofan engine. 


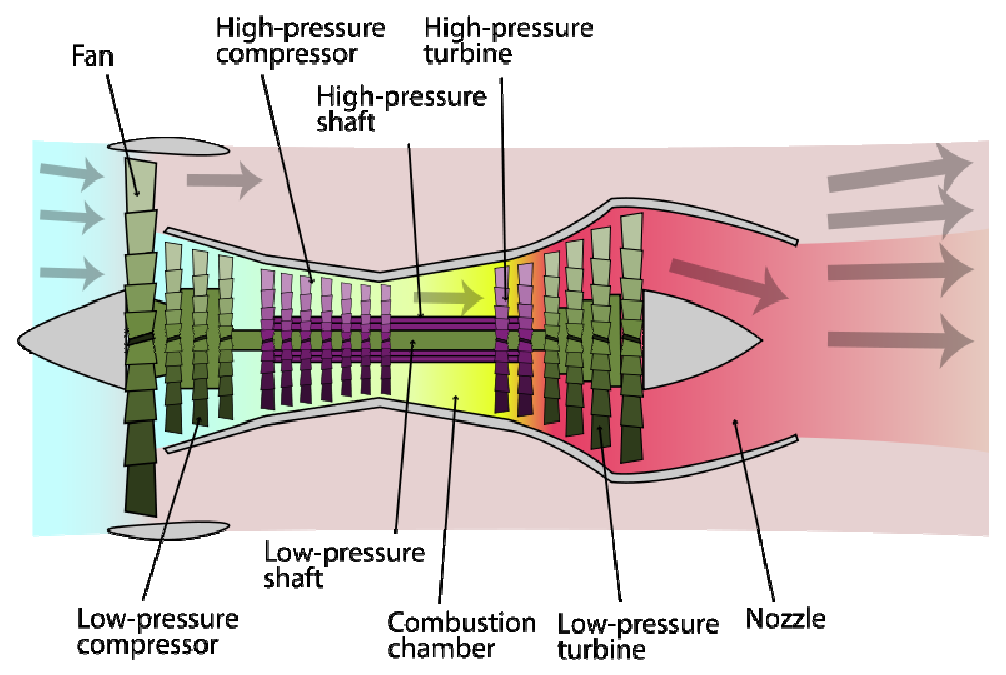

Figure 7. Illustration of a Two-spool Turbofan Engine ${ }^{v}$.

In this figure the high and low-pressure spools are colored purple and green, respectively. This design allows the two spools to spin at different speeds; they are decoupled. The high-pressure spool, due to its smaller diameter and high workload compressing the air before the combustor and extracting energy after, operates more efficiently at high rotational speeds. The low-pressure spool on the other hand must rotate at much slower speeds due to the large diameter fan which produces the majority of the engine's thrust. Because the high-pressure spool prefers to operate at high speed, and the fan prefers low speed, the two decoupled spools allow these components to operate at their preferred speeds nearer to their peak efficiencies. Unfortunately, as can be seen in Figure 7, the low-pressure spool also has a compressor and turbine in addition to the fan. Since this compressor and turbine of the low-pressure spool have a much smaller diameter than the fan, they too prefer to spin at higher speeds, somewhere between the speed of the fan and the speed of high-speed compressor. Thus, there is a tradeoff between operating at the fan's peak efficiency or at the low-pressure compressor and turbine's peak efficiency. This tradeoff also exists to a lesser degree in the high-pressure spool since these compressor and turbine stages also vary in diameter.

The solution is to either design the fan, compressor, and turbine stages as well as possible such that they operate efficiently even when not at their optimal rotating speeds, or add more spools. Unfortunately, fan, compressor, and turbine stage design has arguably reached a 
pinnacle in terms of aerodynamics and efficiency. Except for improvements made possible by advanced materials, there is little advancement to be made with these components. The other option, adding more spools, has been explored by Rolls-Royce in their newer Trent 1000 and Trent XWB. These turbofan engines features three spools; low, intermediate, and high-pressure. Even more spools could improve engine performance and efficiency, but mechanical complexity rises exponentially with each additional spool while the improvements in performance increase only marginally. The Figure 8 shows a two-spool CFM International CFM56-7B turbofan (left) and a three-spool Rolls-Royce Trent 1000 turbofan.
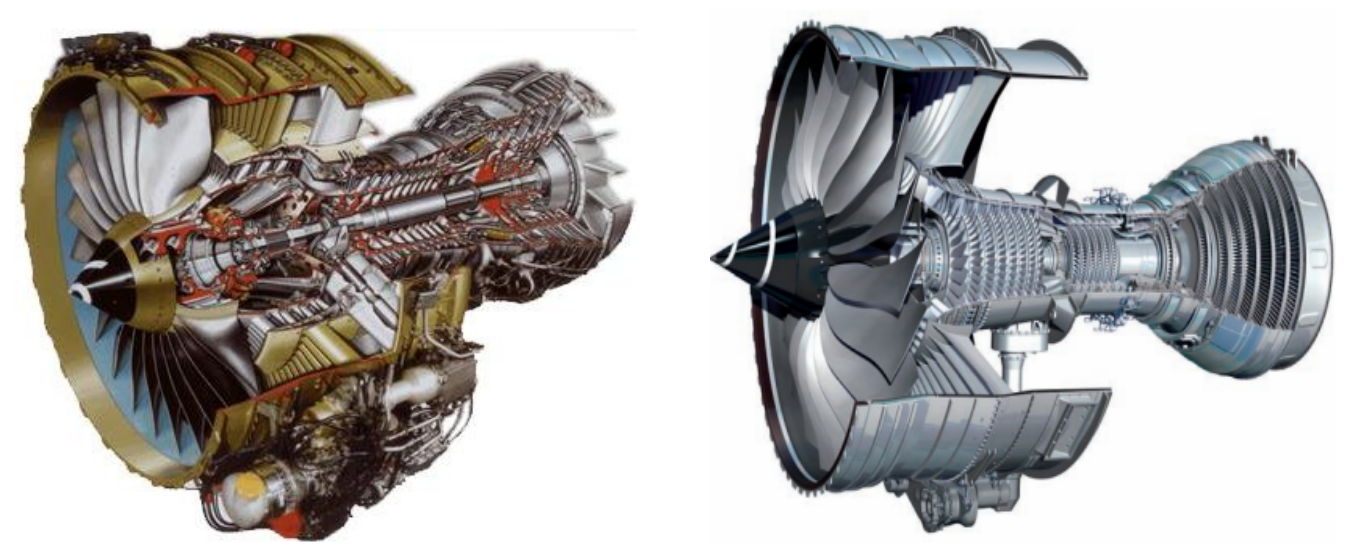

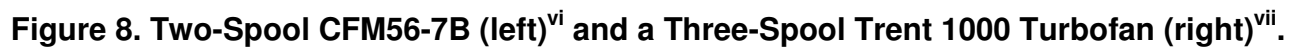

No matter how many spools are added to the engine, the fan (thrust producer) is always coupled to the turbine (power producer) via a fixed mechanical shaft, and these two components are forced to operate at the same rotational speeds. In a pure series hybrid aircraft propulsion system, the fan and turbine are decoupled via the electrical system. Thus, the fan is free to operate at whatever speed desired as long as there is electrical power available, and the turbine can operate at its desired speed as long as the power it is generating can be consumed or stored.

In essence, the electrical portion of the hybrid system becomes an "infinite gearbox" (or an "infinite-spool" engine relating back to turbofans). The hybrid system transfers power from engine to the propulsor while still allowing both to operate at any feasible speed. And as a result, the engine and propulsor can both operate at their peak efficiencies for much more of the mission, dramatically improving propulsive efficiency and thermal efficiencies. 


\subsubsection{Component Lapse}

Yet another significant advantage of aircraft hybrid propulsion is the fact that electrical components such the motor, generator, and batteries do not lapse. Lapse, a term most commonly used for engines, quantifies the engine's deviation in output power/thrust from its "standard" value. The standard value is typically measured at sea-level standard day conditions at zero airspeed. There are two main causes of engine lapse; variations in air density due to altitude, temperature, and pressure, and the effect of flight speed on air entering the engine.

The amount of power/thrust an engine can produce depends directly on the mass of air the engine is able to ingest. At high altitudes the air density is reduced from that at sea level, leading directly to a reduction in air mass that the engine is able to ingest. This ultimately results in a reduced maximum possible output power and the engine is said to have lapsed.

A second cause of lapse is flight speed. Because the aircraft is traveling with forward speed, the air also enters the inlet of the engine with some speed. However, this air is typically decelerated before entering the first compression stage of the engine, effectively raising the pressure of the air before it is even ingested. This is called "ram effect" and increases the output power capability of the engine, also considered lapse. Figure 9 shows the performance map from a Honeywell TPE331-10 turboprop engine. 


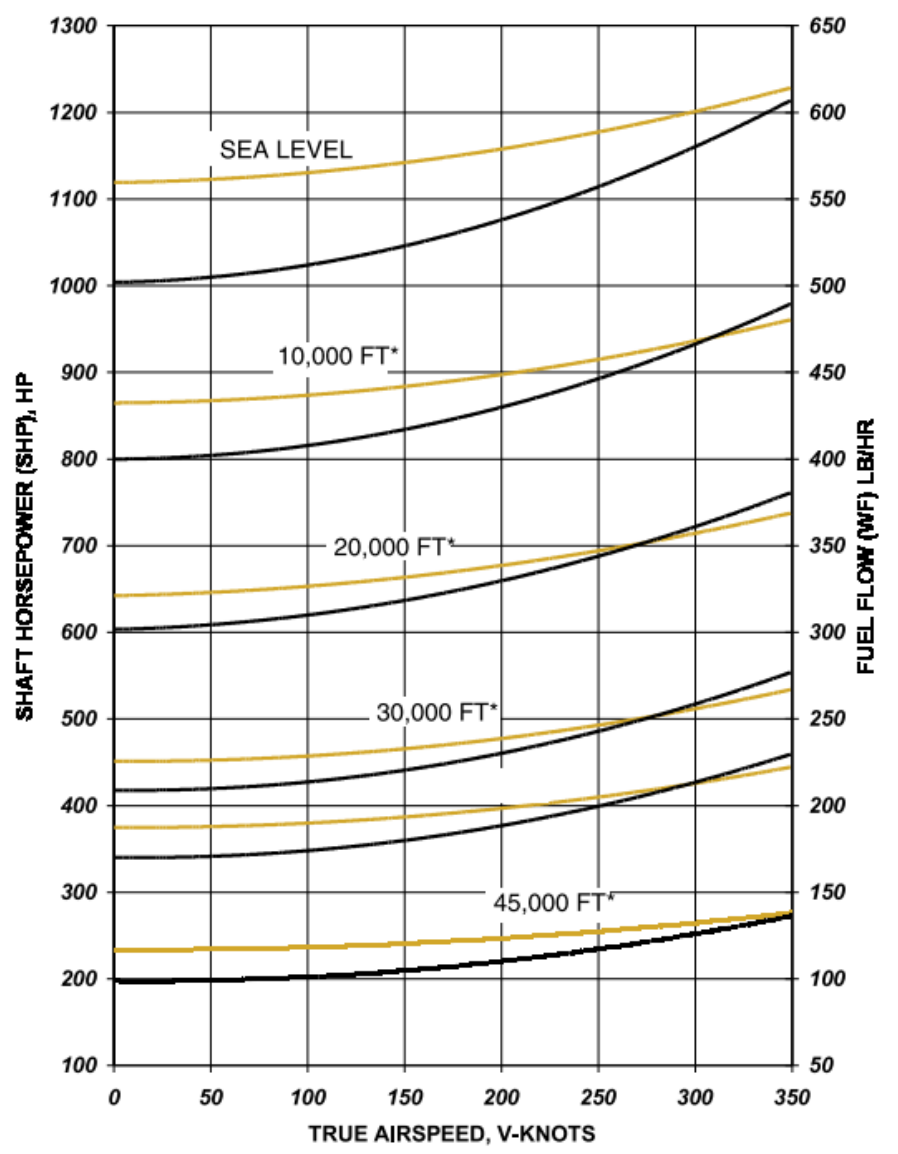

Figure 9. Honeywell TPE331-10 Turboprop Performance Map ${ }^{\text {viii }}$.

In this figure the yellow lines represent fuel flow while the black lines represent engine output power. At sea level and zero airspeed, this engine is capable of outputting $1000 \mathrm{hp}$. At $45,000 \mathrm{ft}$ and zero airspeed however, the engine is only capable of outputting $200 \mathrm{hp}$. This reduction in power is called lapse. In this case, the engine has lapsed $80 \%$ which corresponds almost directly with the decrease in air density of $80.6 \%$ between sea level and $45,000 \mathrm{ft}$. This figure also shows the increase in output power due to airspeed. At sea level for example, the output power of this engine increases from $1000 \mathrm{hp}$ at zero air speed to $1210 \mathrm{hp}$ at $350 \mathrm{kts}$. In this case the engine has lapsed $121 \%$.

Now consider this engine at a cruise condition of $30,000 \mathrm{ft}$ and $350 \mathrm{kts}$ where its maximum output power is $550 \mathrm{hp}$. This engine has lapsed $45 \%$ from the sea level static condition due to the reduced density of the atmosphere. And as a result air-breathing engines sized to provide adequate power at the cruise condition are typically overpowered at sea level. Lapse is a phenomenon that plagues all air-breathing engines, but not electrically powered devices such as 
motors, generators, and batteries. These machines do not consume air and thus are not affected by changes in air density.

In the aforementioned situation where the air-breathing engine is overpowered at sea level, the excess headroom in the engine could be used to charge batteries in a hybrid propulsion system during take-off and climb. When the aircraft reaches the cruise condition, the batteries could provide supplemental power to the electric propulsors reducing the fuel consumption. Of course, the charging and discharging schedule of the batteries depends highly on the configuration and the goal of the vehicle. In some configurations it may be preferable to discharge the batteries during take-off and climb and charge during cruise. The flexibility in the charge/discharge schedule is yet another significant advantage of hybrid aircraft propulsion systems.

\subsubsection{Effective Bypass Ratio}

The bypass ratio of a turbofan engine is defined as the ratio between the mass flow rate of air passing through the fan (aka secondary or cold section) and the mass flow rate of air passing through the core (aka primary or hot section). Generally speaking, the higher the bypass ratio, the more efficient and quieter the engine. Using the examples from Figure 8, the CFM56 and Trent 1000 engines have bypass ratios of approximately 5.5 and 11, respectively. Higher bypass ratio turbofans however require larger fan diameters with lower fan rotational speeds, and thus more spools/gearing to achieve the proper core rotational speeds. There also exist complicated design challenges at near-transonic speeds with high bypass ratio turbofans. The mechanical complexity of turbofan engines rises rapidly with bypass ratio, and the Rolls-Royce Trent 1000 turbofan is bumping into the feasible upper limit. Lastly, high bypass ratio turbofans tend to be very large in diameter. This leads to increased engine weight, longer and heavier landing gear to fit the engines under the wing, and greater nacelle weight and drag.

By decoupling the propulsor fans from the power producing engine, and distributing many smaller fans across the aircraft, the effective bypass ratio can be increased greatly. In the hybrid aircraft propulsion system, the effective bypass ratio is defined as ratio between the total mass flow rate of air passing through all of the propulsor fans and the total mass flow rate of air passing 
through all of the internal combustion engines. The effective bypass ratio of a hybrid aircraft propulsion system can be several times that of conventional turbofan engines. The ECO-150 from Figure 6 had a conservative effective bypass ratio of 22.39. Even higher bypass ratios above 30 are possible with more aggressive hybrid distributed systems.

\subsubsection{Aircraft Control \& Transients}

Distributed propulsion using a hybrid system has the added benefit of being able to provide aircraft control via the distributed propulsors. For example, to provide a yawing moment, the power to the distributed propulsors on the one side of the wing would be increased, while the other side is decreased. Depending on the location of the propulsors they could also provide pitching and rolling moments via clever throttling.

This is difficult to achieve in contemporary turbofan aircraft for a couple of reasons. First, the time required for a turbofan engine to respond to a throttle input (transient) is generally quite long since the rotational components of a turbofan have a high rotational inertia, especially in today's larger high-bypass ratio engines. When a substantial amount of throttle is demanded suddenly, several seconds are required for a turbofan to speed-up and produce more thrust. With a hybrid system, the power for the electric motors driving the propulsive fans is readily available via the batteries. In a distributed system where there are many small propulsors, each fan/motor combination has a much smaller rotational inertia, and can thus react to a throttle input and accelerate much more quickly. Electric motors also provide very high torque, allowing them to overcome the inertia of the fan with less effort.

A second reason why a distributed hybrid system is beneficial to aircraft control deals with the location of the propulsors and their effective moment arm. For example, traditional turbofan engines are hung from the wing at approximately 1/3 span. With a distributed system there is the ability to place smaller propulsors further out on the wing, up to $1 / 2$ span if not further. A single propulsor placed at 2/3 span would have the same yawing moment arm on the aircraft as a turbofan with double the thrust placed at $1 / 3$ span. A greater moment arm allows for greater aircraft control. 
If the pilot has a greater ability to control the aircraft by tailoring the thrust of the individual propulsors, the control surfaces and empennage could effectively be reduced in size as well as their associated hydraulic systems and structure. The weight savings of such would be on the order of hundreds if not thousands of pounds for larger aircraft.

\subsubsection{One Engine Inoperative}

Today's commercial transport aircraft must meet strict regulations regarding one engine inoperative (OEI) conditions. These regulations, described in FAR part $25.121^{\text {ix }}$, state that the aircraft must maintain a specific climb gradient during takeoff. This gradient depends on the number of engines and the takeoff segment, the strictest of which is the second segment. During second segment climb at full takeoff weight with the landing gear retracted and OEI, the aircraft must be able to climb with a gradient of $2.4 \%$ for two-engine aircraft, $2.7 \%$ for three-engine aircraft, and $3.0 \%$ for four-engine aircraft. This second segment OEI climb is classically the most limiting constraint on aircraft thrust-to-weight ratio, causing the engines to be oversized.

With a hybrid aircraft propulsion system utilizing distributed propulsors, the OEI climb becomes a much more trivial concern. Using the ECO-150 from Figure 6 for example, if one of the 16 propulsors becomes inoperative during takeoff, the total thrust of the aircraft is only reduced by $6 \%$, as opposed to $50 \%$ for a two-engine aircraft. The electric motors in the propulsors can also be designed to overspeed and produce any extra thrust required to complete the second segment climb. Using the ECO-150 again as an example, if one of the two turboshaft engines failed during takeoff, the extra power required could be provided from the batteries. In the unlikely event that both turboshaft engines fail, the aircraft could still fly on batteries alone long enough to make an emergency landing. If one turboshaft engine was to fail, assuming the electrical power can be redistributed between the fans, the loss in thrust is usually only $30-40 \%$, instead of $50 \%$ like a twin-turbofan aircraft.

With the added redundancies of a hybrid system, the second segment OEI climb becomes much less of a constraint and the propulsion system can be sized much smaller and lighter. Granted FAR 25 applies to modern turbofan powered aircraft, and would need to be modified for hybrid propulsion systems. 


\subsubsection{Maintenance}

In the event that a propulsor does fail, the time and manpower required to replace one would be minimal. Each propulsor would have just electrical and cooling connections, attached to the airframe or wing structure with just a handful of hardware. The total weight of each propulsor would be fairly low and could be removed with a small lift or hoist depending on its size. Replacing a propulsor could be performed in under an hour with crew of just a few mechanics.

Compare this to a typical turbofan engine which has a myriad of electrical connections, oil, cooling, and fuel lines, as well as ducting for environmental control systems and anti-icing. These engines also weigh several thousand pounds and require specialized lifts to remove. Replacing a turbofan engine is no trivial task and results in days of aircraft downtime.

\subsubsection{Descent Power \& Windmilling}

With traditional turbofan powered aircraft, the engines are throttled down to a flight idle setting for descent. Even at flight idle, turbofan engines are still capable of producing several thousand pounds of residual thrust because of the need to avoid flameout. A flameout occurs when the volume of air passing through the combustion chamber is too great for the amount of fuel being injected, causing the burner flame to extinguish. For this reason drag producing devices such as speed brakes and spoilers are typically required to counteract the residual thrust and sufficiently slow the aircraft for approach.

With a TeDP system however, the electricity-producing turboshaft engines create little residual thrust even at high throttle settings. For this reason the turboshaft engines could continue to operate at full power during descent to charge the batteries in preparation for terminal operations or the next flight. The thrust producing propulsors on the other hand could be throttled down to whatever setting desired, or shut down completely. If shut down, the propulsors could act as generators due to the windmilling effect, also helping to charge the batteries. When windmilling, the propulsors themselves would create a significant amount of drag, likely eliminating the need for speed brakes or spoilers. 


\subsubsection{CG Excursion}

In contemporary aircraft, the fuel is primarily stored in the wing and in the fuselage near the wing carry-through structure. Some aircraft such as the Boeing 747 have fuel located in the empennage as well. As the aircraft flies the mission, the fuel is consumed and the center of gravity (CG) of the aircraft shifts either forward or aft, called CG excursion. To counteract the shifting of the CG, fuel management systems pump fuel between tanks. The horizontal stabilizer is also used to neutralize the pitching moment caused by the shifting CG, inducing trim drag. Due to the significant mass of fuel consumed during a mission, the CG excursion can be considerable. The Boeing 747-400ER for example holds an immense 425,000 lbs of fuel, most of which can theoretically be consumed during a mission.

In a hybrid aircraft propulsion system, some of the fuel mass that would normally be required to complete a mission is exchanged for batteries. Unlike with fuels, as the energy in batteries is expended, the mass of the battery does not change. This property of batteries provides some advantage in regards to CG excursion. Depending on the hybrid system and aircraft configuration, a heavy complicated fuel management system may not be required if the CG excursion is kept to a minimum. The trim drag during cruise could also be reduced.

\subsection{Disadvantages of Hybrid Aircraft Propulsion}

\subsubsection{Electrical Component Weight}

With a hybrid aircraft propulsion system, the total propulsion group weight is likely to be greater than that of a traditional turbofan aircraft. The primary reason for this is the added weight of the battery packs, which overall have a much lower specific energy than kerosene-based fuels. The batteries also have significantly lower energy density (energy per unit volume) than fuels, which can be problematic when trying to locate batteries in the aircraft.

Furthermore, the power density of electric generators and motors tend to be lower than that of turbine engines. The Honeywell TPE331-10 turboshaft for example has a power density of $2.60 \mathrm{hp} / \mathrm{lb}$. Larger turboshaft engines such as the Rolls-Royce T406, which powers the BellBoeing V-22 Osprey, has an impressive power density of $6.33 \mathrm{hp} / \mathrm{lb}$. Contemporary electric 
generators and motors however have power densities around $2 \mathrm{hp} / \mathrm{lb}$. The electric machines must be sized larger than their turbine-based counterparts in order to provide the same amount of power, which leads to increased propulsion system weight. Foreseeable advances in motor technology including cryogenic cooling, new stator/rotor designs, and control techniques could dramatically improve the power density of electric machines.

A third aspect of the hybrid system weight is a counterargument to the advantage presented in section 2.3.9. In a traditional aircraft the fuel is consumed throughout the mission and the vehicle becomes lighter, requiring less and less power to propel the vehicle and to stay airborne. In a hybrid system however, the mass of the battery does not change as it expels energy. If the battery is completely drained of energy, the aircraft must still transport the battery throughout the duration of the mission.

\subsubsection{Electrical Inefficiencies}

A significant disadvantage of hybrid aircraft propulsion systems is that they introduce many more inefficiencies into the propulsion system. In a traditional turbofan powered aircraft, the propulsion system inefficiencies exist primarily inside the turbofan itself; in the compressor, combustor, turbine, fan, inlet, exhaust nozzle, etc. A hybrid system is also burdened with these turbine engine inefficiencies, as well as inefficiencies introduced by the electrical components.

The electric generators, batteries, controllers, motors, and cabling all have inefficiencies associated with them. When combined in a pure series fashion, these inefficiencies multiply and the result can be significant losses in power. Today's best performing electric motors and generators have peak efficiencies of about $95 \%$ which stems primarily from electrical resistances and iron losses. Cryogenic cooling systems however could significantly decrease the electrical resistance allowing for efficiencies greater than $99 \%$. While cryogenic cooling technology for aircraft systems is still several decades in the future, it is a technology which could virtually eliminate electrical inefficiencies.

In non-cryogenic (conventional/warm) systems, additional cooling would be required for the electrical components. Due the immense amount of power required for a large aircraft to take flight, conventional electric components would require complex and robust cooling systems. 


\subsubsection{System Complexity}

Undoubtedly, a hybrid aircraft propulsion system would be far more complex and intricate than a typical turbofan powered aircraft. For a hybrid propulsion system to be effective, it must be integrated very tightly with the aircraft itself. This is true for both the additional electrical cabling which must be strung throughout the vehicle, and the electric propulsors which would ideally be distributed throughout the aircraft and even integrated into the structure.

With added complexity also comes an increased chance of component failure. Although a hybrid propulsion system inherently has many redundancies, additional fail-safes would need to be integrated into the system.

\subsection{In Conclusion}

The primary advantage of a hybrid aircraft propulsion system is not necessarily to increase the efficiency of the propulsion system, but to allow the vehicle to fly more "smartly". With the additional weight and inefficiencies introduced by a hybrid propulsion system, the efficiency of converting fuel into thrust is in fact reduced. For example, the Honeywell TPE331-10 turboshaft engine referenced earlier can convert the chemical energy in the fuel to useful mechanical energy with an efficiency of about $40 \%$ (at sea level static maximum power). By introducing a generator, controller, and motor in series, each with an efficiency of $95 \%$, the system efficiency is reduced by about $14 \%$.

By decoupling of the power producer from the thrust producer, the fuel consuming and electricity consuming devices can operate nearer their peak efficiency for a greater period of time. The addition of a battery allows for electrical power to be supplied to or absorbed from the system whenever required, increasing the flexibility of the system. For this reason the overall vehicle efficiency during a mission can be dramatically increased.

Hybrid propulsion can also produce a significant increase in overall vehicle efficiency. Other advantages such as aircraft control via distributed propulsors, reduction in CG excursion during flight, descent power and drag generation, and general vehicle maintenance also provide an overall "smarter" vehicle which can operate more intelligently during its mission and lifecycle. 


\section{Overview of HAPSS}

\subsection{Introduction}

Hybrid Aircraft Propulsion System Synthesis (HAPSS) is a program written in the MATLAB language and designs and analyzes hybrid propulsion systems for aircraft. This program focuses solely on pure series hybrid systems; a fuel-consuming engine mechanically linked to an electric generator, electricity distribution between the generator, battery, and motor managed by a central controller, and a propulsive fan mechanically linked to an electricity-consuming motor to provide thrust. Although the program has been written for this specific type of system, the code architecture is generic enough to allow other modules to be created to model virtually any type of hybrid system. The overarching goal of the program is to identify potential performance benefits of aircraft hybrid systems while exposing unforeseen weaknesses.

The principle function of HAPSS is to size the various components of the hybrid system given user-defined inputs and requirements, and then predict off-design performance of the system. HAPSS provides very detailed point performance, or in other words calculates the performance of the vehicle and the hybrid propulsion system at a single flight condition. With relative ease, a mission analysis tool can be incorporated with HAPSS to calculate overall mission performance.

HAPSS was created with the need and goals of Empirical Systems Aerospace ${ }^{x}$ in mind. The idea of HAPSS began in early 2010 during NASA SBIR contract NNX10CC81Piv " The Design and Integration of a Distributed Fan Propulsion System within a Split-Wing." During this contract, a fan design tool was created to analyze fan performance for the TeDP ECO-150 shown earlier in Figure 6. This work was presented at NASA Glenn in July 2010. After the conclusion of this SBIR contract in July 2010, the actual HAPSS program began by utilizing the fan design tool as a starting point. The other necessary components of the hybrid propulsion system were added into the program allowing for a comprehensive analysis of an aircraft hybrid system. The fan design portion of the code was also expanded to permit greater user flexibility. 


\subsection{System Diagram}

Before HAPSS could be started, a generic hybrid system architecture had to be conceived. The architecture had to be both simple enough to encode and analyze, but detailed enough to provide a valuable study of the hybrid system. Figure 10 shows a diagram of the generic pure series hybrid system that was chosen to be analyzed in HAPSS.

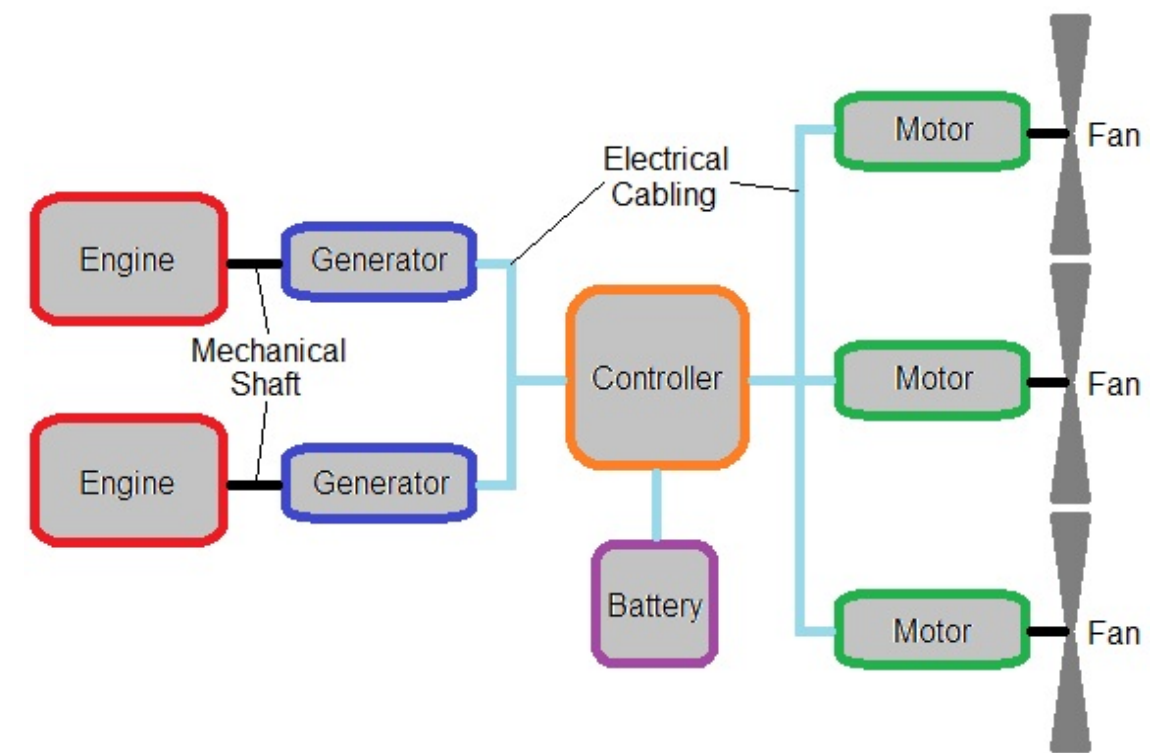

Figure 10. Generic Pure Series Hybrid System Diagram for HAPSS.

In this figure, there are two fuel-consuming engines and three thrust-producing electric propulsors. HAPSS however is capable of modeling any number of engines/generators and propulsors as requested by the user. To simplify the analysis, HAPSS models the system using just a single controller and a single battery pack. Although the real system is likely to have a myriad of controllers and battery packs, this level of detail was too great for the program in this stage. The controller and battery configuration is a much greater facet of detailed system design and aircraft integration. And in reality, the number and configuration of controllers and batteries would not have a significant impact on the system performance.

\subsection{Logic Flow}

The overall flow of logic through the program is fairly simple and linear. A basic diagram illustrating this flow is shown in Figure 11. 


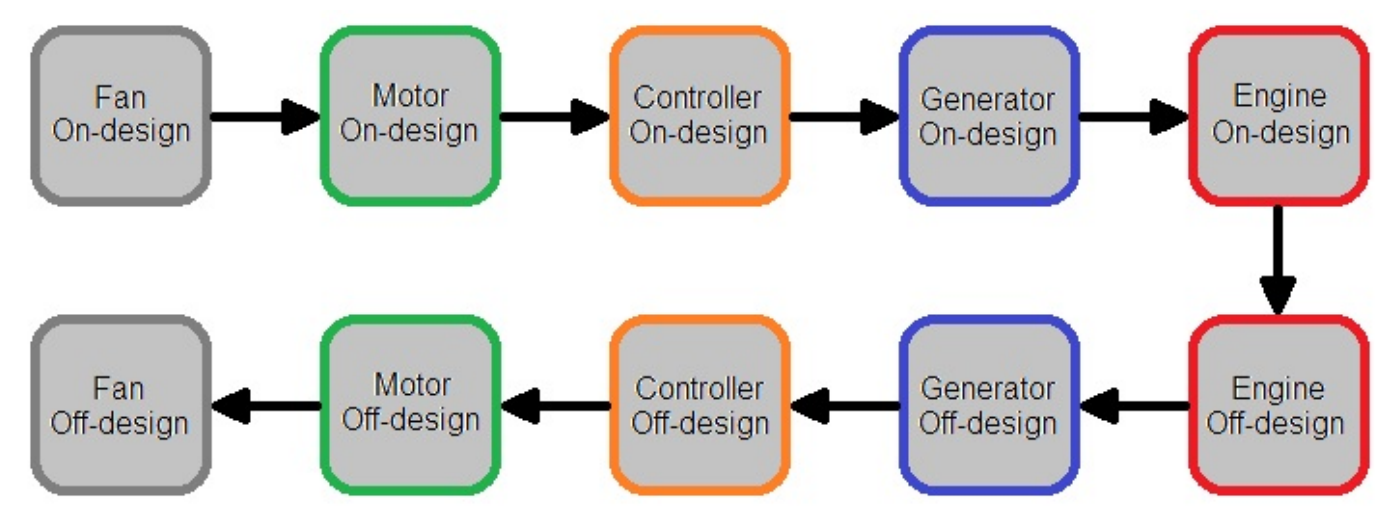

Figure 11. Logic Flow Diagram of HAPSS.

The code begins with the user-defined inputs at the design point of the aircraft. The design point is typically the most stringent and demanding flight condition that the aircraft would ever experience, and for this reason a single design point commonly used to size the propulsion system. Using the inputs from this point, the fan is sized and its power required is determined.

The electrical system (motor, generator, and controller) will typically have a different design point than the aircraft. The reason for this, described in section 2.3.3, is that the electrical components do not lapse like engines. The most demanding flight condition for the electrical system will frequently be takeoff at sea level where the engines themselves are capable of producing their maximum power. However, with the introduction of batteries, which can be used to provide supplemental power at any flight condition, this may not always be the case. It is the user's responsibility to choose the design points appropriately and provide reasonable inputs at these points. If the electrical design point is poorly chosen, the results from HAPSS will reflect this as an over-powering of the electrical components.

With the electrical system sized at the electrical design point, and the power required by the fan calculated at the aircraft design point, the performance of the electrical system is determined whilst considering the battery behavior. This allows for the sizing of the engine and the determination of its performance. At this point, the components of the hybrid propulsion system have been sized at the design point(s).

To determine off-design performance, HAPSS follows a similar process but in reverse. First, the power available from the engine is determined at the off-design point. While considering 
the power provided/absorbed by the battery, the performance of the electrical system is determined next. Finally, the power available to the motor can be resolved which allows for the calculation of thrust produced by the fan.

While each calculation block in Figure 11 seems fairly simple and straightforward, each requires complex calculations and often iterative loops to converge upon the correct solution. Figure 12 shows a more detailed logic flow diagram of HAPSS.

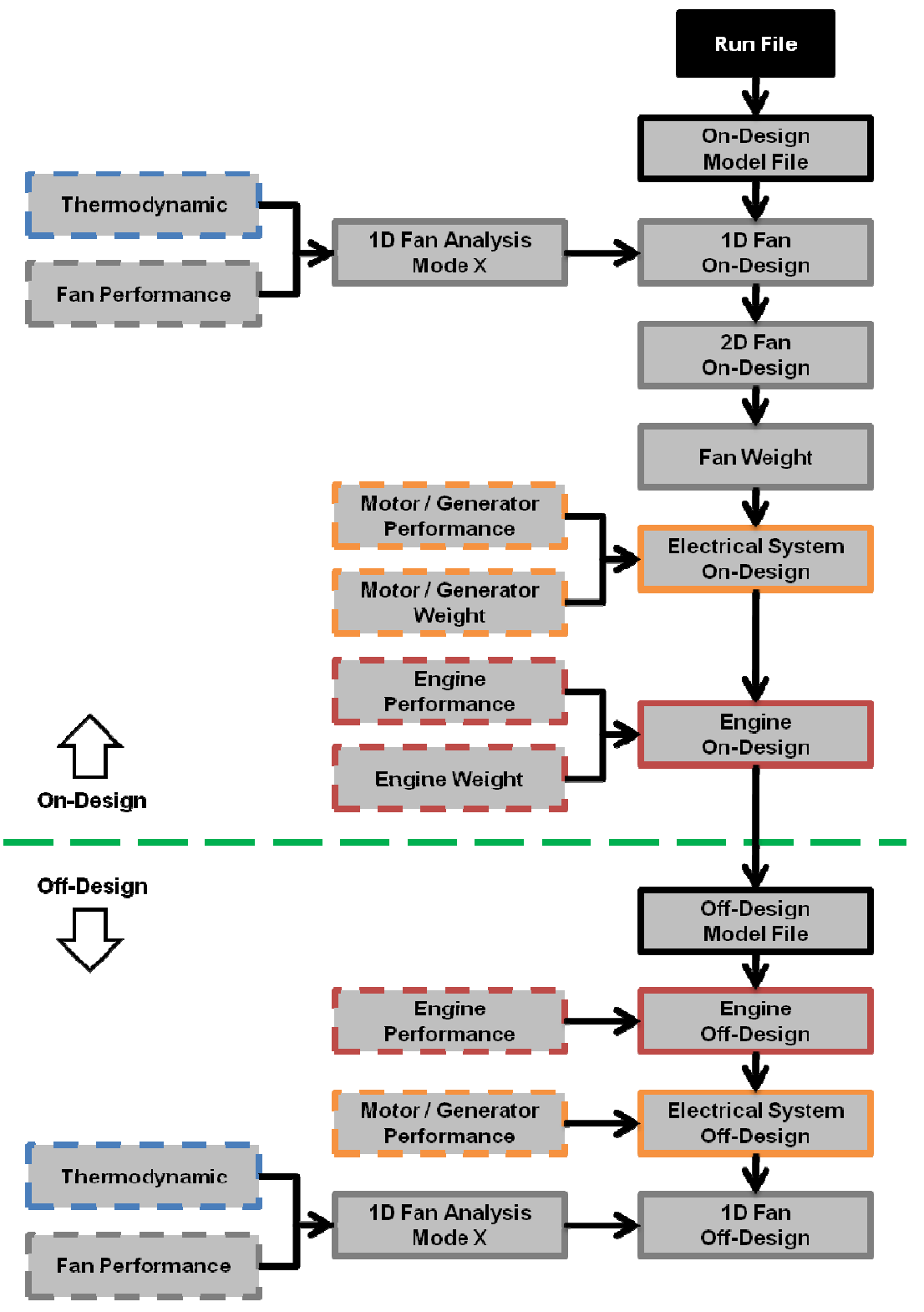

Figure 12. Detailed Logic Flow Diagram of HAPSS. 
In this figure, each block represents a "module", which can be a single or multiple MATLAB scripts or functions. The blocks with dashed borders are MATLAB functions, while those with solid borders are MATLAB scripts. For example, there is a single "Fan On-Design" script, but 14 different "Thermodynamic" functions. Some of these modules require iterative solutions while others can be completed with a linear straightforward approach.

HAPSS makes heavy use of both MATLAB scripts and functions. "Scripts are simply files containing a sequence of MATLAB statements. Functions make use of their own local variables and accept input arguments ${ }^{\mathrm{xi}}$." Functions are useful when statements need to be executed multiple times such as in an iterative loop, while scripts are useful for when the statements only need to be executed once in a linear fashion. To date, HAPSS consists of approximately 60 MATLAB files (excluding post-processing scripts) and about 3,500 lines of code.

\subsection{Modules \& Functions}

This section briefly describes each module and function in Figure 12. The modules for the off-design condition are analogous to those of the on-design since the analyses are very similar, but in reverse order. The off-design modules do not calculate component weights and dimensions however since these are fixed from the on-design condition.

- Run File: This script is a master executable script that calls the various sub-modules in the correct order. It also allows the user to select whether or not to run off-design cases. Typically there will be a script higher than this one to manipulate inputs and record outputs for a multitude of design and off-design conditions.

- Model File: This script contains all of the inputs necessary for the on and off-design analyses. This is the file in which the user sets the specifications for the fan, electrical system, engine, etc. This file also contains the names of the various performance and weight functions which are used throughout the analyses.

- 1D Fan: This script first verifies all the inputs for the 1D fan performance analysis. If the user provides fixed value inputs, this script will confirm that these values are within an appropriate range. If the user neglects to provide an input, this script recalls the default value. If fan 
performance functions are utilized instead of fixed inputs, this script resolves these variables. This script then assembles the input data structure for the 1D fan performance analysis code and executes the correct fan analysis mode.

- 1D Fan Analysis, Mode X: These are the core scripts for calculating the $1 \mathrm{D}$ fan performance. There currently are 11 different scripts, each of which determines fan performance utilizing different inputs. For example, the user may select one mode over another depending on what inputs are available to the user. Of the 11 modes, 7 require iterative solutions and have their own dedicated functions (not pictured in Figure 12) for doing so.

- Fan Performance: These functions can be created by the user to calculate certain inputs for the 1D fan performance analysis. For example, if the inlet pressure recovery is known to be a function of the flight Mach number and altitude, a function for this can be created and easily utilized in the code. These functions are called by the 1D Fan Analysis, Mode X script.

- Thermodynamic: The thermodynamic functions were created to calculate very basic thermodynamic properties and processes. The property function calculates specific heat, molecular weight, enthalpy, entropy, and more of a fluid given temperature and fuel-air ratio. The process functions calculate thermodynamic processes such as isentropic compression and expansion given a variety of inputs. There are 14 different process functions. There is also a standard atmosphere function to calculate important thermodynamic data based on altitude and a temperature offset. These functions are also called by the 1D Fan Analysis, Mode X script.

- 2D Fan: This script calculates the 2D performance of the fan at the mean fan diameter. It determines the velocity triangles including absolute, relative, and whirl velocities, Mach numbers, and diffusion factors. This script then performs a top-level 2D blade cross section design.

- Fan Weight: This script predicts the weight of the fan assembly using methodology developed for the NASA WATE-1 ${ }^{\text {xvi }}$ (Weight Analysis of Turbine Engines) program. 
- Electrical System: Similar to the other scripts, this file first checks all of the inputs supplied by the user to ensure they are valid, or calculates inputs if the user supplies functions. This script then calculates the various aspects of the electrical system including motor and generator sizes and efficiencies, controller input and output powers, battery charging and discharging, and heat lost in the electrical system.

- Motor/Generator Performance: These functions are similar to the fan performance functions where the various aspects of the electrical system can be defined by the use with functions instead of being fixed. For example, the motor and generator efficiencies will typically be defined using functions. These functions can also be used to input the power and torque characteristics of the electric machines. These functions are called by the Electrical System script.

- Motor/Generator Weight: This is a script with calculates the weight of the motor and generator based on their maximum output power. This function is also called by the Electrical System script.

- Engine: This script calculates the performance of the engine including output power, power lapse, power specific fuel consumption (PSFC), fuel flow rate, efficiency, and weight using a user-inputted weight function.

- Engine Performance: The engine function uses engine data to calculate the engine lapse and PSFC for varying altitude, flight speed, and throttle setting.

- Engine Weight: This function is used to predict the weight of the engine using its maximum output power at sea level static. 


\section{On-Design Methodology}

This section describes the methodology and approach taken for each module during the ondesign analysis. Figure 13 shows the HAPSS flow diagram but with the lower off-design portion blurred. Section 4 will focus on the upper on-design portion of this flow chart.

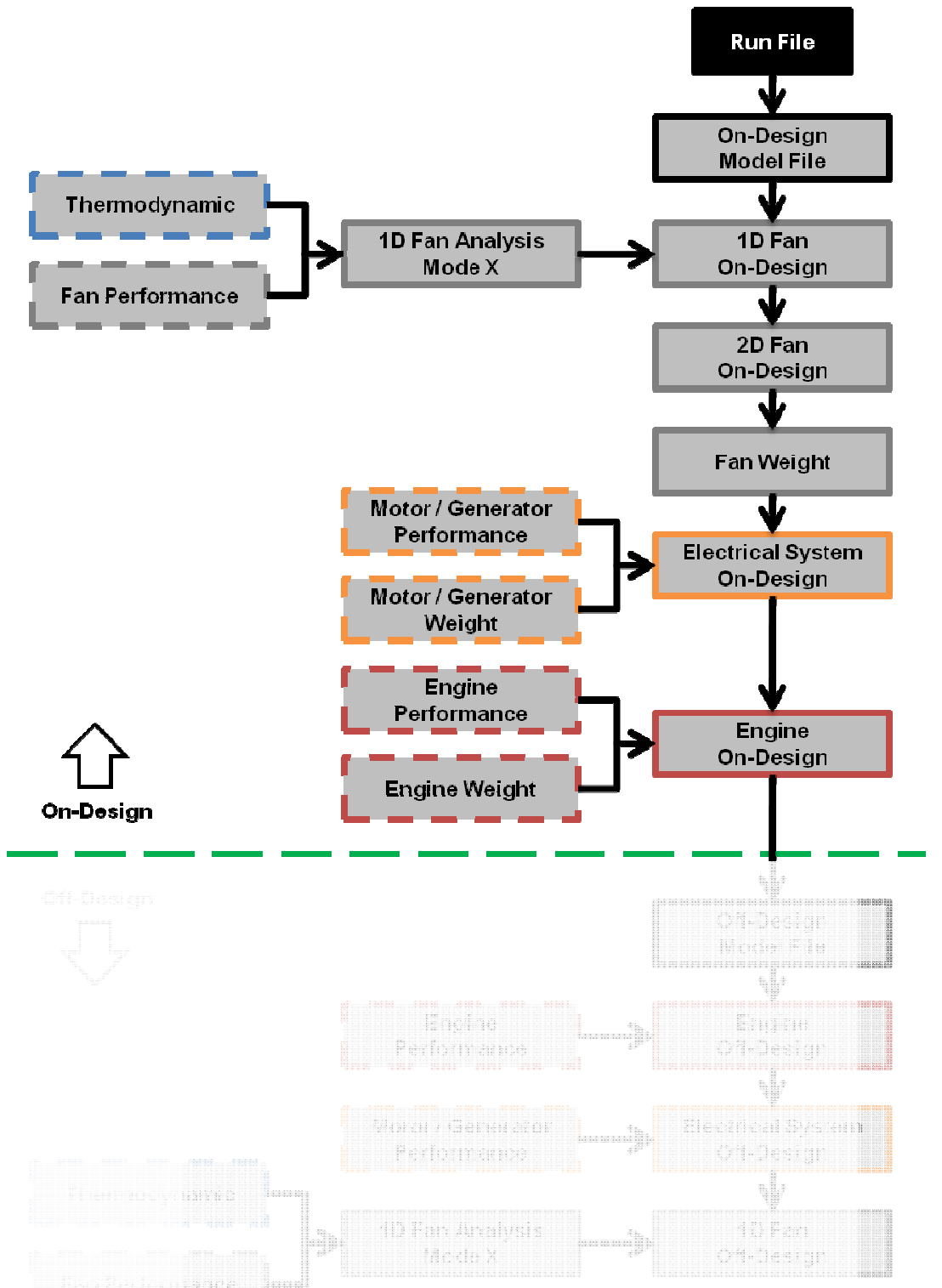

Figure 13. Detailed On-Design Logic Flow Diagram of HAPSS.

Some modules in this flow chart are used only to call other MATLAB scripts and functions or manipulate inputs, while others perform detailed sizing and performance calculations of the 
hybrid propulsion system. The detail provided in this section will be more focused on the latter module type.

\subsection{Run File}

The run file can be considered the master executable script which calls the various modules in the correct order. It also allows the user to select whether or not to run the off-design case. Typically there will be a script higher than this one to manipulate inputs and record outputs for a multitude of design and off-design conditions. The basics of the run file are shown here.

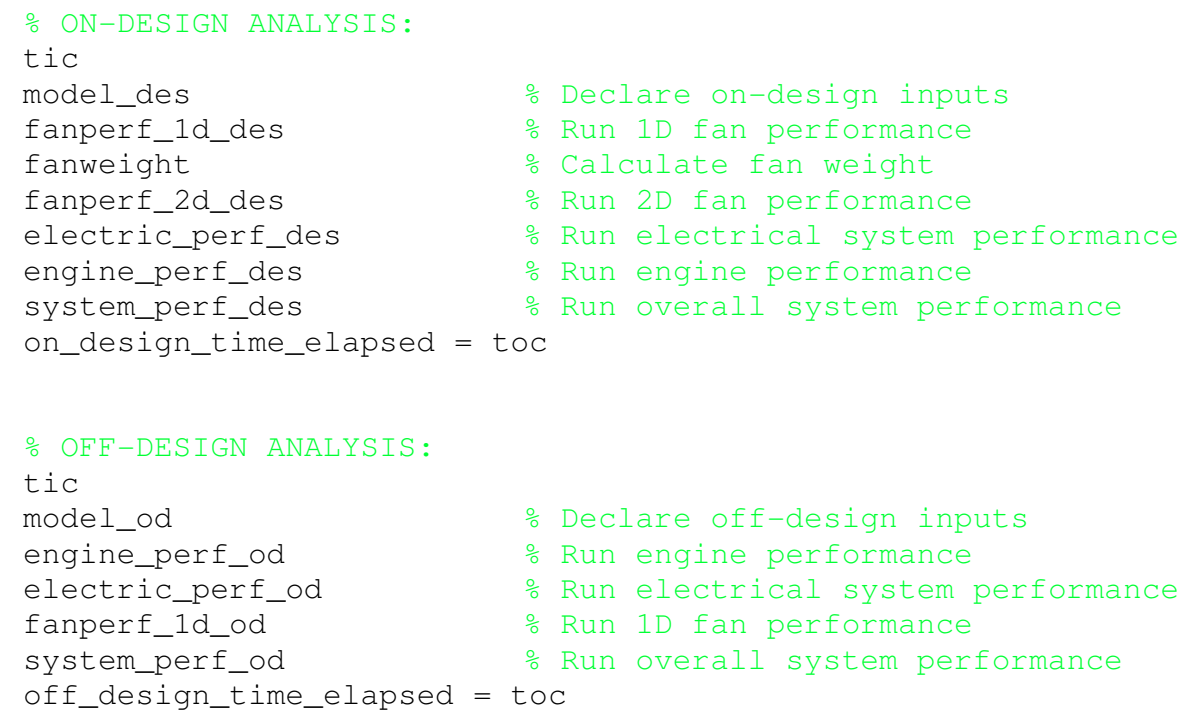

The run file first performs the on-design analysis to size the hybrid propulsion system. It starts by calling the on-design model file contains all of the user inputs to the program. Next, the fan performance modules are executed to calculate fan performance at the design condition followed by the fan weight. The electrical system is then sized followed by the engines. The offdesign analysis follows a similar order but in reverse. This script also records the elapsed time to complete each analysis.

\subsection{On-Design Model File}

This script contains all of the inputs required to execute the on-design analysis. These inputs, and all of the variables used in HAPSS, are stored in MATLAB data structures. Data 
The checking of the inputs follows a hierarchical approach. If the user defines an input using a performance function then the value of the input is calculated overwriting any other entry for that variable. For example, if the user defines the inlet pressure recovery, $P R 2$, to be 0.98 , and also inputs a function for the inlet pressure recovery, PR2fun, the inlet pressure recovery is calculated and the 0.98 is ignored. HAPSS is currently setup to accept functions for most of the 1D on-design fan variables, however these functions can only accept flight Mach number, altitude, and power setting as inputs at this time. An excellent example of this is defining the inlet pressure recovery as a function of flight Mach number and altitude. This script also checks if the function is valid and errors out if not.

If a function for a variable is not defined, then the script checks if the variable was inputted at all. If not then a default value is used. If the variable was inputted, the script checks to see if it falls within in reasonable bounds, erroring out if it does not. An example of this input checking is shown here for inlet pressure recovery.

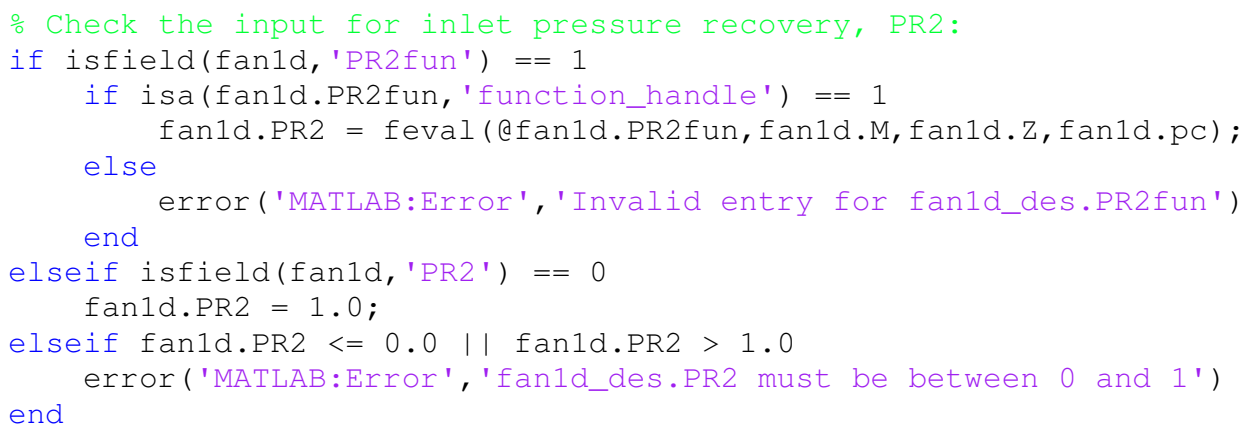

With each input checked/calculated this script then calls the correct 1D fan performance mode, described in section 4.5 .

\subsection{Thermodynamic}

Before looking at the 1D fan analysis modes, it's important to understand the thermodynamic functions that support them. The thermodynamic functions set is comprised of 16 separate functions, each of which resolve various thermodynamic properties or analyze processes. These calculations oftentimes require an iterative approach so these functions were created to separate the analysis into manageable pieces. 


\subsubsection{Thermodynamic Properties}

This first function calculates thermodynamic properties of the working fluid including specific heat, enthalpy, entropy, gas constant, molecular weight, ratio of specific heats, and the weight flow function based on properties such as temperature, fuel/air ratio, and/or Mach number. The inputs and outputs of this function are summarized Table 1.

Table 1. Inputs and Outputs of the Thermodynamic Properties Function.

\begin{tabular}{|c|c|c|c|l|}
\hline Output & \multicolumn{3}{|c|}{ Inputs } & \multicolumn{1}{c|}{ Description } \\
\hline $\boldsymbol{c}_{\boldsymbol{p}}$ & $T$ & far & & Specific heat at constant pressure \\
\hline $\boldsymbol{W}$ & $f$ & & & Molecular weight \\
\hline $\boldsymbol{h}$ & $T_{2}$ & $T_{1}$ & $f$ & Enthalpy difference between 1 and 2 \\
\hline $\boldsymbol{s}$ & $T_{2}$ & $T_{1}$ & $f$ & Entropy difference between 1 and 2 \\
\hline $\boldsymbol{V}$ & $T$ & $f a r$ & & Ratio of specific heats \\
\hline $\boldsymbol{w f f}$ & $M$ & $T$ & $f$ & Weight flow function based on Mach number \\
\hline
\end{tabular}

The specific heat at constant pressure is derived using reaction stoichiometry which takes into account the various constituents of the air (nitrogen, oxygen, argon, and carbon dioxide) as well as the post-combustion fuel-to-air ratio. The derivation of this equation can be found in NASA-TM-X-1340 $0^{\mathrm{xii}}$ and the coefficients come from NASA SP-3001 $1^{\mathrm{xiii}}$.

$$
\begin{gathered}
c_{p}=\frac{\sum\left(\bar{W} c_{p}\right)_{\text {components }}}{1+f} \\
c_{p}=\left(\frac{1}{1+f}\right)\left\{0.24062-0.017724\left(T * 10^{-3}\right)+0.038056\left(T * 10^{-3}\right)^{2}-0.012662\left(T * 10^{-3}\right)^{3}+\right. \\
0.0013012\left(T * 10^{-3}\right)^{4}+\left[0.22091+0.51822\left(T * 10^{-3}\right)-0.19462\left(T * 10^{-3}\right)^{2}+0.045089(T *\right. \\
\left.\left.\left.10^{-3}\right)^{3}-0.0043275\left(T * 10^{-3}\right)^{4}\right] * f\right\}
\end{gathered}
$$

where $T$ is the air temperature in ${ }^{\circ} \mathrm{R}$ and $f$ is the fuel-to-air ratio. Although in the majority of situations the propulsive fan will be subject to zero fuel-to-air ratio air, it was desired to use this equation for possible future expansion of the code to include a turbine engine module.

The enthalpy and entropy equations equation also account for the fuel-to-air ratio and calculate the difference between state 1 and state 2 as shown in the following equations.

$$
\Delta h=\int_{T_{1}}^{T_{2}} c_{p} d T
$$




$$
\begin{gathered}
\Delta h=\left(\frac{1}{1+f}\right)\left\{0.24062\left(T_{2}-T_{1}\right)-\frac{0.017724 * 10^{-3}}{2}\left(T_{2}^{2}-T_{1}^{2}\right)+\frac{0.038056 * 10^{-6}}{3}\left(T_{2}^{3}-T_{1}^{3}\right)-\frac{0.012662 * 10^{-9}}{4}\left(T_{2}^{4}-\right.\right. \\
\left.T_{1}^{4}\right)+\frac{0.0013012 * 10^{-12}}{5}\left(T_{2}^{5}-T_{1}^{5}\right)+\left[0.22091\left(T_{2}-T_{1}\right)+\frac{0.51822 * 10^{-3}}{2}\left(T_{2}^{2}-T_{1}^{2}\right)-\frac{0.19462 * 10^{-6}}{3}\left(T_{2}^{3}-T_{1}^{3}\right)+\right. \\
\left.\left.\frac{0.045089 * 10^{-9}}{4}\left(T_{2}^{4}-T_{1}^{4}\right)-\frac{0.0043275 * 10^{-12}}{5}\left(T_{2}^{5}-T_{1}^{5}\right)\right] * f\right\} \\
\Delta s=\int_{T_{1}}^{T_{2}} \frac{c_{p}}{T} d T \\
\begin{array}{c}
\Delta s=\left(\frac{1}{1+f}\right)\left\{0.24062 \ln \frac{T_{2}}{T_{1}}-0.017724 * 10^{-3}\left(T_{2}-T_{1}\right)+\frac{0.038056 * 10^{-6}}{2}\left(T_{2}^{2}-T_{1}^{2}\right)-\frac{0.012662 * 10^{-9}}{3}\left(T_{2}^{3}-\right.\right. \\
\left.T_{1}^{3}\right)+\frac{0.0013012 * 10^{-12}}{4}\left(T_{2}^{4}-T_{1}^{4}\right)+\left[0.22091 \ln \frac{T_{2}}{T_{1}}+0.51822 * 10^{-3}\left(T_{2}-T_{1}\right)-\frac{0.19462 * 10^{-6}}{2}\left(T_{2}^{2}-T_{1}^{2}\right)+\right. \\
\left.\left.\frac{0.045089 * 10^{-9}}{3}\left(T_{2}^{3}-T_{1}^{3}\right)-\frac{0.0043275 * 10^{-12}}{4}\left(T_{2}^{4}-T_{1}^{4}\right)\right] * f\right\}
\end{array}
\end{gathered}
$$

To calculate the enthalpy or entropy of a simple fluid the initial temperature, $T_{1}$, in these equations would simply be zero. The molecular weight is calculated using the following equation which also takes into consideration the fuel-to-air ratio.

$$
\begin{gathered}
W=\frac{1+f}{\Sigma\left(\frac{W}{M}\right)_{\text {components }}} \\
W=\frac{1+f}{0.034522+0.035648 f}
\end{gathered}
$$

With the molecular weight determined, the ratio of specific heats can then be calculated using the following equation.

$$
\gamma=\frac{1}{1-\frac{\bar{R}}{W J c_{p}}}
$$

where $\bar{R}$ is the universal gas constant of $1545 \mathrm{ft}-\mathrm{Ib}_{\mathrm{f}} /\left(\mathrm{Ibmol}-{ }^{\circ} \mathrm{R}\right)$ and $J$ is the conversion factor of $778.16 \mathrm{ft}-\mathrm{lb}_{\mathrm{f}} / \mathrm{BTU}$.

This function also calculates a very useful parameter called the weight flow function. This function is a derivative of the fundamental continuity equation and is useful in HAPSS for calculating flow area based on total conditions. The weight flow function is constant for isentropic flow and is shown here with the continuity equation for reference. 


$$
\begin{gathered}
\dot{m}=\rho A V \\
w f f=\frac{\dot{m} \sqrt{T}}{A P}=\sqrt{\sqrt{\frac{\gamma g_{c}}{R} / W}}\left(1+\frac{\gamma-1}{2} M^{2}\right)^{-\frac{\gamma+1}{2(\gamma-1)}}
\end{gathered}
$$

where $\dot{m}$ is the mass flow rate is $\mathrm{lb}_{\mathrm{m}} / \mathrm{sec}, T$ is the total temperature in ${ }^{\circ} \mathrm{R}, A$ is the flow area in $\mathrm{ft}^{2}$, $P$ is the total pressure in $\mathrm{lb}_{\mathrm{f}} / \mathrm{ft}^{2}, g_{c}$ is the gravitational constant of $32.174 \mathrm{lb}_{\mathrm{m}}-\mathrm{ft} /\left(\mathrm{lb}_{\mathrm{f}}-\mathrm{s}^{2}\right)$, and $M$ is the flow Mach number.

As shown in Table 3, the inputs for this function are either temperature, $T$ in ${ }^{\circ} \mathrm{R}$, fuel-to-air ratio, far, and/or Mach number, $M$. The main purpose of this function is to support the next set of thermodynamic functions which analyze processes. When this function is called, the user specifies which output to be calculated and provides the required inputs in the order shown in Table 1. For example, if the weight flow function is desired for standard air at $519{ }^{\circ} \mathrm{R}$ traveling at Mach 0.2, the function is called in MATLAB as follows. The weight flow function for this example is 0.1795 .

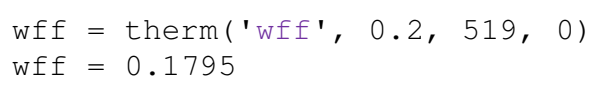

\subsubsection{Thermodynamic Processes}

The next set of thermodynamic functions resolve processes including isentropic and polytropic expansion and compression based on parameters such as efficiency, fuel/air ratio, pressure ratio, and enthalpy change. There are 14 of these functions where each one analyzes a specific process given certain inputs. The inputs and outputs of these functions are summarized in Table 2. 
Table 2. Inputs and Outputs of the Thermodynamic Process Functions.

\begin{tabular}{|c|c|c|c|c|c|c|}
\hline \# & Output & & & & & Description \\
\hline 1 & $\begin{array}{c}T_{2} \\
\Delta h\end{array}$ & $P_{2} / P_{1}$ & $T_{1}$ & far & $\eta_{s}$ & Isentropic compression \\
\hline 2 & $\begin{array}{c}T_{2, i} \\
T_{2} \\
\Delta h\end{array}$ & $P_{1} / P_{2}$ & $T_{1}$ & far & $\eta_{s}$ & Isentropic expansion \\
\hline 3 & $\begin{array}{c}T_{2, i} \\
T_{2} \\
P_{1} / P_{2}\end{array}$ & $\Delta h$ & $T_{1}$ & far & $\eta_{s}$ & Isentropic expansion \\
\hline 4 & $\begin{array}{c}C_{p 1} \\
Y \\
R \\
h_{1} \\
\end{array}$ & $T_{1}$ & far & & & Basic thermodynamic calculations \\
\hline 5 & $\begin{array}{c}Y \\
R \\
\text { wff } \\
A / A^{*} \\
\end{array}$ & $T_{1}$ & far & $M$ & & Computes flow function \\
\hline $\begin{array}{l}6 \\
7\end{array}$ & $\frac{\mathrm{far}_{2}}{T}$ & $\begin{array}{l}T_{1} \\
h_{1}\end{array}$ & $\frac{T_{2}}{f a r}$ & LHV & $\eta_{\text {comb }}$ & $\begin{array}{l}\text { Computes fuel/air ratio in combustor } \\
\text { Computes temperature }\end{array}$ \\
\hline 8 & $\begin{array}{c}\eta_{s} \\
T_{2, i} \\
T_{2} \\
P_{1} / P_{2} \\
\end{array}$ & $\Delta h$ & $T_{1}$ & far & $\eta_{p}$ & Isentropic expansion \\
\hline 11 & $\begin{array}{c}\eta_{s} \\
T_{2, i} \\
T_{2} \\
\Delta h\end{array}$ & $P_{2} / P_{1}$ & $T_{1}$ & far & $\eta_{p}$ & Isentropic compression \\
\hline 12 & $\begin{array}{c}T_{2, i} \\
T_{2} \\
P R\end{array}$ & $\Delta h$ & $T_{1}$ & far & $\eta_{s}$ & Isentropic compression \\
\hline 13 & $\begin{array}{c}\eta_{s} \\
T_{2, i} \\
T_{2} \\
P_{2} / P_{1}\end{array}$ & $\Delta h$ & $T_{1}$ & far & $\eta_{p}$ & Isentropic compression \\
\hline 14 & $\begin{array}{c}V \\
T / t \\
P / p \\
\end{array}$ & $T$ & far & M & & Computes isentropic relations \\
\hline 15 & $\begin{array}{c}\eta_{s} \\
T_{2, i} \\
T_{2} \\
\Delta h \\
\end{array}$ & $P_{1} / P_{2}$ & $T_{1}$ & far & $\eta_{p}$ & Isentropic expansion \\
\hline 16 & $\begin{array}{c}Y \\
T / t \\
P / p \\
\end{array}$ & $T$ & far & V & & Computes isentropic relations \\
\hline
\end{tabular}

These functions were created to model compression and expansion of air when either the polytropic or isentropic efficiency of the process is known. The process can be defined by either a pressure ratio or change in enthalpy of the fluid during the process. Many of these processes 
require an iterative solution because the ratio of specific heats, $\gamma$, changes with temperature. The compression and expansion processes use the following equation to calculate the change in entropy during the process.

$$
\Delta s=\int_{T_{1}}^{T_{2}} c_{p}(T) \frac{d T}{T}-R \ln \frac{p_{2}}{p_{1}}
$$

This equation applies to both ideal compression and ideal expansion properties. The change in enthalpy during the process is estimated using the following equation.

$$
\Delta h=\frac{\Delta h_{i}}{\eta_{s}}
$$

where $\Delta h_{i}$ is the ideal enthalpy change during the process calculated in equation 4.4.1.X and $\eta_{s}$ is the process isentropic efficiency. The isentropic and polytropic efficiencies are related in the following equation.

$$
\eta_{s}=\frac{\left(\frac{p_{2}}{p_{1}}\right)^{\frac{\gamma-1}{\gamma}-1}}{\left(\frac{p_{2}}{p_{1}}\right)^{\frac{\gamma-1}{\gamma \eta}-1}}
$$

There are also functions to calculate the isentropic relations, weight flow function, fuel-toair ratio, and other thermodynamic properties. Although not all of these functions are required for HAPSS, they were created for possible future expansion of the program. These functions are called in a similar fashion as the thermodynamic properties function. For example, if the user wishes to know the pressure ratio of an isentropic compression of standard air with a change in enthalpy of $100 \mathrm{BTU} / \mathrm{lb}_{\mathrm{m}}$, an initial temperature of $519{ }^{\circ} \mathrm{R}$, with a polytropic efficiency of $90 \%$, the function is called in MATLAB as follows.

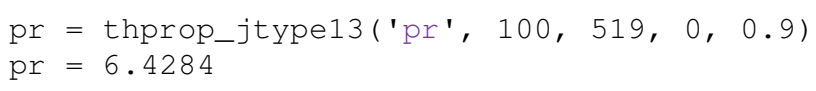

This function calculated a pressure ratio of 6.4284 for this process. In this example, 'pr' flags the function to output pressure ratio. These output flags vary by function.

\subsubsection{Standard Atmosphere}

Finally, a standard atmosphere function was developed to calculate pertinent atmospheric conditions at the given flight conditions. This function uses the U.S. Standard Atmosphere, 1976 model $^{\mathrm{xiv}}$ to calculate atmospheric properties up to $65,000 \mathrm{ft}$. Up to this altitude, the International Standard Atmosphere and MIL-STD Standard Atmosphere models are identical. 
This standard atmosphere function was used only in the 1D and 2D fan analysis, but could also be utilized in additional modules in the future. The following equation defines the sea-level reference temperature.

$$
T_{0}^{\prime}=T_{0}+\Delta T
$$

where $T_{0}$ is the standard day sea-level reference temperature of $518.7{ }^{\circ} \mathrm{R}\left(59^{\circ} \mathrm{F}\right)$ and $\Delta T$ is the temperature offset from standard day in ${ }^{\circ} \mathrm{R}$. This equation allows for the modeling of nonstandard atmospheres such as hot and high, 4K/95, and 6K95.

In the Troposphere, from sea-level to $36,089 \mathrm{ft}$, the temperature is calculated using the following equation.

$$
T=T_{0}^{\prime}+a Z
$$

where $a$ is the temperature lapse rate of $-0.003566{ }^{\circ} \mathrm{R} / \mathrm{ft}$ and $Z$ is the altitude in $\mathrm{ft}$. In the stratosphere, the temperature is calculated as follows.

$$
T=390+\Delta T
$$

Atmospheric pressure is independent of temperature and thus does not change with temperature offset. The pressure in the troposphere and stratosphere is shown in Equations 0x and $0 \mathrm{x}$, respectively.

$$
\begin{gathered}
P=P_{0}\left(1+\frac{a Z}{T_{0}}\right)^{5.2561} \\
P=P_{0}\left(0.2234 e^{-\frac{Z-36089}{20806.7}}\right)
\end{gathered}
$$

where $P_{0}$ is the standard day sea-level reference pressure of $2116.2 \mathrm{lb}_{f} / \mathrm{ft}^{2}$. The density of the can be calculated using the perfect gas equation shown here.

$$
\rho=\frac{P}{g R T}
$$

where $g$ is the acceleration of gravity of $32.17 \mathrm{ft} / \mathrm{s}^{2}$ and $R$ is the gas constant of $53.35 \mathrm{ft} /{ }^{\circ} \mathrm{R}$. Although these values vary slightly with altitude and temperature, they are fixed in this atmospheric model. The dynamic viscosity is calculated using Sutherland's formula shown here.

$$
\mu=\mu_{0}\left(\frac{T}{T_{0}}\right)^{1.5}\left(\frac{T_{0}+198.72}{T+198.72}\right)
$$

where $\mu_{0}$ is the dynamic viscosity at sea-level of $3.62 \times 10^{-7} \mathrm{Ib}_{\mathrm{m}}-\mathrm{s} / \mathrm{ft}^{2}$. The kinematic viscosity, $v$, is calculated simple by dividing the dynamic viscosity by the density. The last values calculated by 
this function are the temperature $(\theta)$, pressure $(\delta)$, and density $(\sigma)$ ratios. These are calculated simply by dividing the temperature, pressure and density at altitude by the reference values at sea-level.

The following example shows the output from the function executed at $30,000 \mathrm{ft}$ on a standard day (zero temperature offset).

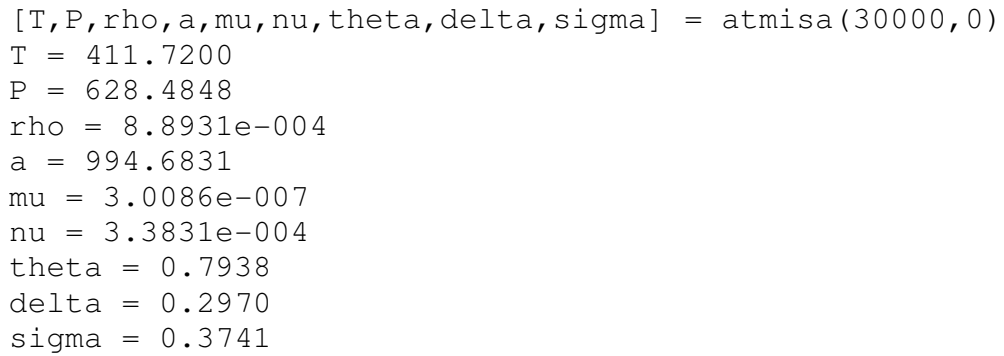

\subsection{D Fan Analysis, Mode X}

In order to determine the performance of the fan, it is the user's responsibility to supply enough information such that the analysis can be performed. However, the fan information that the user has available may vary depending on the situation. To account for this, multiple scripts were created, each of which approaches the fan analysis differently depending on which inputs are available. In HAPSS, each approach is called a "mode" and has its own script file. There are six main fan design variables which the user has the option to input or output depending on the mode.

- $\quad$ Pressure ratio, $\pi_{f}$

- $\quad$ Thrust, $F$

- Mass flow rate, $\dot{m}_{f}$

- Fan diameter, $D_{f}$

- $\quad$ Power, Pwr

- Fan face Mach number, $M_{2}$

All other fan design variables are either always inputted or always outputted. Generally speaking, three of these six variables are required as inputs to resolve the fan performance. There are therefore several dozen combinations of these variables each of which could have its 
own dedicated mode. However, after listing all of the possible combinations, it became apparent that some were less important than others. For example, rarely would the user know the mass flow rate through the fan, and thus most of these modes were disregarded. While the difference in calculations between each mode was minimal, it was decided to separate the modes regardless to simplify the coding. A total of 12 modes were created and are listed here in Table 3.

Table 3. 1D Fan Performance Modes.

\begin{tabular}{|c|c|c|c|c|c|c|c|c|c|c|c|c|}
\hline Mode & 1 & 2 & $3^{\wedge}$ & $4^{\star}$ & 5 & $6^{\wedge}$ & $7^{\wedge}$ & 8 & $9^{\wedge}$ & $10^{\wedge \wedge}$ & $11^{\wedge}$ & $12^{\wedge \wedge}$ \\
\hline$\pi_{f}$ & in & in & in & var & in & out & out & in & out & out & in & out \\
\hline$F$ & in & out & out & in & out & out & in & out & in & in & in & out \\
\hline$\dot{m}$ & out & out & out & out & in & out & out & out & out & out & out & out \\
\hline$D_{f}$ & out & out & in & out & out & in & out & in & in & in & in & in \\
\hline$P w r$ & out & in & in & $\min$ & out & in & in & out & out & in & $\overline{\text { out }}$ & in \\
\hline$M_{2}$ & in & $\overline{\text { in }}$ & out & in & in & in & $\overline{\text { in }}$ & in & in & out & out & out \\
\hline
\end{tabular}

In this table "in" denotes that the parameter must be inputted by the user while "out" denotes that the parameter is outputted by the mode. Of these 12 modes, only modes $1,2,5$, and 8 can be solved linearly without any iterating. All of the iterative modes use either mode 1 or 2 to perform the analysis starting with an initial guess for one of the parameters, typically pressure ratio or fan face Mach number. Mode 5 is the only one with mass flow rate as an input and was created for validation purposes.

Mode 4, described in section 4.5.6, is a special case because it varies the fan pressure ratio with the goal of minimizing the power required by the fan, and thus only has two inputs. Mode 12 , described in section 4.5 .12 , also has just two inputs and uses fan affinity coefficients to estimate off-design performance. This mode only works for off-design cases; intuitively the fan performance cannot be resolved from knowing the power and diameter alone.

Before discussing the analysis of the fan, it is important to take a look at the system that will be analyzed. Figure 14 shows an exploded view of a generic propulsor that is analyzed using HAPSS. This figure came from the NASA SBIR ECO-150 analysis performed in $2010^{\mathrm{iv}}$. 


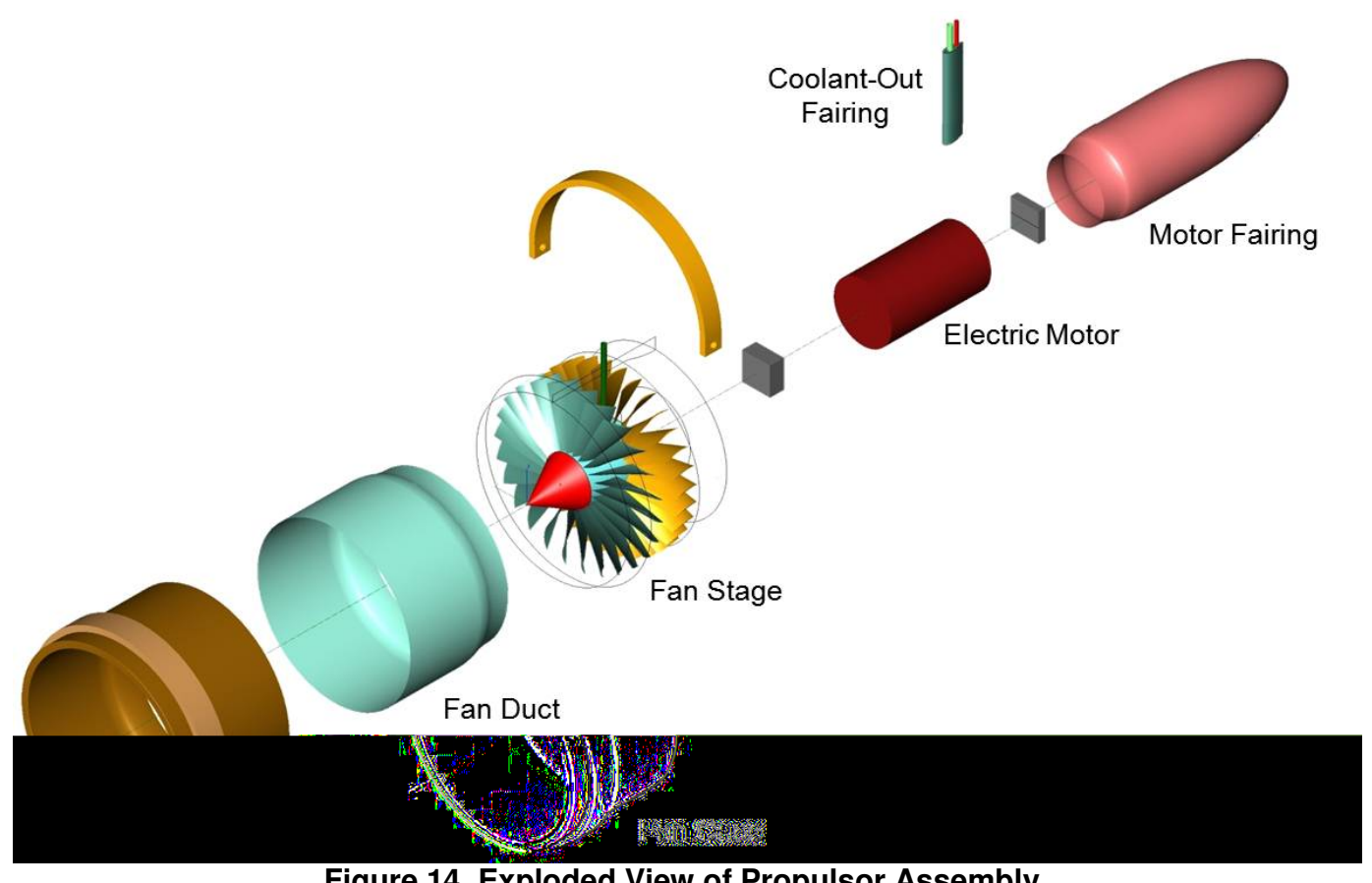

Figure 14. Exploded View of Propulsor Assembly.

This figure shows the fan unit as a single-stage (one rotor and one stator) propulsor. The fan is driven directly by the motor which is encased in a streamlined fairing. The fan is surrounded by a duct and casing for integration with the aircraft. Finally, a coolant fairing is shown which transfers the liquid or air coolant to the motor itself. The electrical cables are assumed to access the motor through one or more of the stator blades.

The analysis performed in HAPSS is only concerned with the fan stage itself. The effect of the casing and fairings are simplified as losses in the inlet and nozzle. The labeled fan stations are shown in Figure 15.

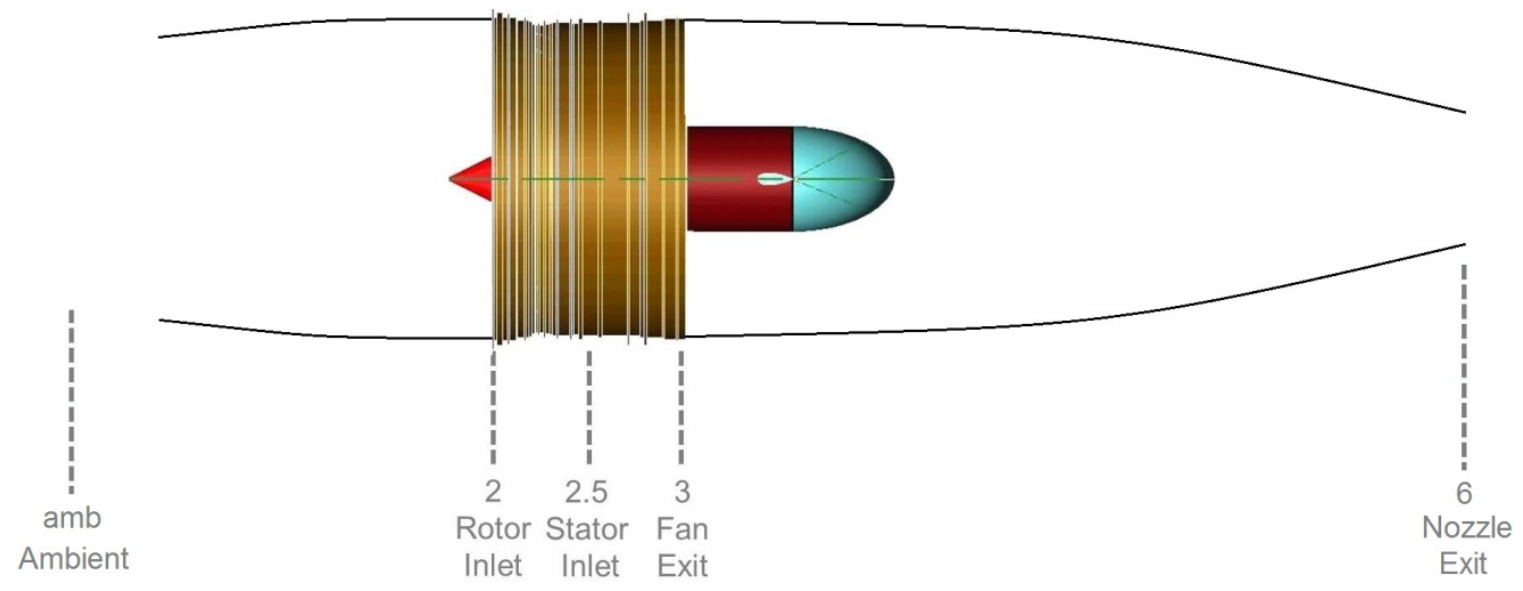

Figure 15. Fan Stations. 
In this figure, "amb" is the ambient air at the flight condition, " 2 " is the fan/rotor face, "2.5" is the rotor exit/stator inlet, " 3 " is the stator exit/exhaust duct inlet, and " 6 " is the nozzle exit. This nomenclature was chosen because it is congruent with common industry notation. This notation also applies to the 2D fan analysis.

The following sections describe the methodology behind each of the modes. The majority of detail will be given to mode 1 since the other modes are only slight alterations of the same methodology. The linear non-iterating modes $1,2,5$, and 8 will be discussed first followed by the rest of the modes in sequential order.

\subsubsection{Mode 1}

This mode of the 1D performance analysis requires the user to input fan pressure ratio, fan face Mach number, and required thrust, and outputs fan diameter, power required, and mass flow rate. This mode would typically be used for sizing the fan at the on-design condition.

\section{Station amb - Ambient}

The fan analysis starts at the ambient station and steps through the propulsor to calculate its final performance. At each station, gratuitous thermodynamic properties are calculated for validation and bookkeeping purposes. The calculation of these properties will also be covered in detail in this section.

The 1D fan analysis begins with the calculation of the atmospheric conditions at the ambient station using the standard atmosphere function described in section 4.4.3. This function uses the altitude and a temperature offset to calculate the static temperature and pressure, density, acoustic speed, dynamic and kinematic viscosities, and the temperature, pressure, and density ratios. The ratio of specific heats is calculated using the thermodynamic properties function described in section 4.4.1. The ratio of specific heats is calculated at each station instead of held constant. The ambient total conditions are calculated using the isentropic relations as shown in the following equations.

$$
T_{a m b}=t_{a m b}\left(1+\frac{\gamma_{a m b}-1}{2} M_{a m b}^{2}\right)
$$




$$
P_{a m b}=p_{a m b}\left(1+\frac{\gamma_{a m b}-1}{2} M_{a m b}^{2}\right)^{\frac{\gamma_{a m b}}{\gamma_{a m b}-1}}
$$

where $\gamma$ is the ratio of specific heats, $T_{a m b}$ and $P_{a m b}$ are the total ambient temperature in ${ }^{\circ} \mathrm{R}$ and pressure in $\mathrm{lb} / \mathrm{ft}^{2}$, respectively, $t_{a m b}$ and $p_{a m b}$ are the static ambient temperature and pressure, respectively, and $M_{a m b}$ is the flight Mach number. The subscript amb is used to denote properties at the ambient station. A subscript 0 is also common in practice but was not used as this is frequently used to denote total conditions. A thermodynamic process function could also be used to calculate these isentropic relations in the previous equations. The total enthalpy is calculated using the $14^{\text {th }}$ thermodynamic process function (refer section 4.4.2).

The static acoustic speed and flight velocity are then calculated using the following relations.

$$
\begin{gathered}
a_{a m b}=\sqrt{\gamma_{a m b} R t_{a m b} g_{c}} \\
V_{a m b}=M_{a m b} a_{a m b}
\end{gathered}
$$

where $a_{a m b}$ is the static acoustic speed $V_{a m b}$ is the flight speed, both in $\mathrm{ft} / \mathrm{s}$. The gas constant, $R$, with units $\mathrm{ft}-1 \mathrm{~b}_{\mathrm{f}} /\left(\mathrm{lb}_{\mathrm{m}}{ }^{-} \mathrm{R}\right)$, depends only on the fuel-to-air ratio and thus remains constant through the propulsor. The total and static densities in $\mathrm{lb}_{\mathrm{m}} / \mathrm{ft}^{3}$ are also calculated for the ambient condition as shown in the following equations.

$$
\begin{gathered}
\rho_{0, a m b}=\frac{P_{a m b}}{R T_{a m b}} \\
\rho_{a m b}=\frac{p_{a m b}}{R t}
\end{gathered}
$$

\section{Station 2 - Rotor Entrance}

The conditions at the fan face, station 2, are calculated next. Due to inefficiencies and friction in the inlet, there is a pressure loss between the ambient condition and the fan face. The total pressure at the fan face is calculated simply as a percentage of the ambient total pressure, as shown in the following equation.

$$
P_{2}=P_{a m b} P R_{2}
$$

where $P R_{2}$ is the pressure recovery in the inlet, typically ranging from 0.90 to 0.99 in practical applications. The pressure recovery can be estimated by knowing the geometric details of the inlet including length, area ratio, diverging angles, lip radius, mass flow rate, and more. In the 
majority of cases however, the user will of HAPSS will not have such detailed knowledge of the inlet, and thus inputting a single pressure recovery factor is sufficient.

Included in HAPSS is a fan performance function (described briefly in section 3.4) to calculate the pressure recovery based on the flight Mach number. In reality, the inlet pressure recovery depends on much more than the just flight Mach number, however this approach provides reasonable accuracy at this time. The user can also create his or her own function to calculate the inlet pressure recovery if more accuracy is desired. The included pressure recovery function, named PR2_parabolic, uses a parabolic curve to approximate the pressure recovery between the static condition and a critical Mach number. Figure 16 illustrates subcritical and supercritical inlet flow phenomena.
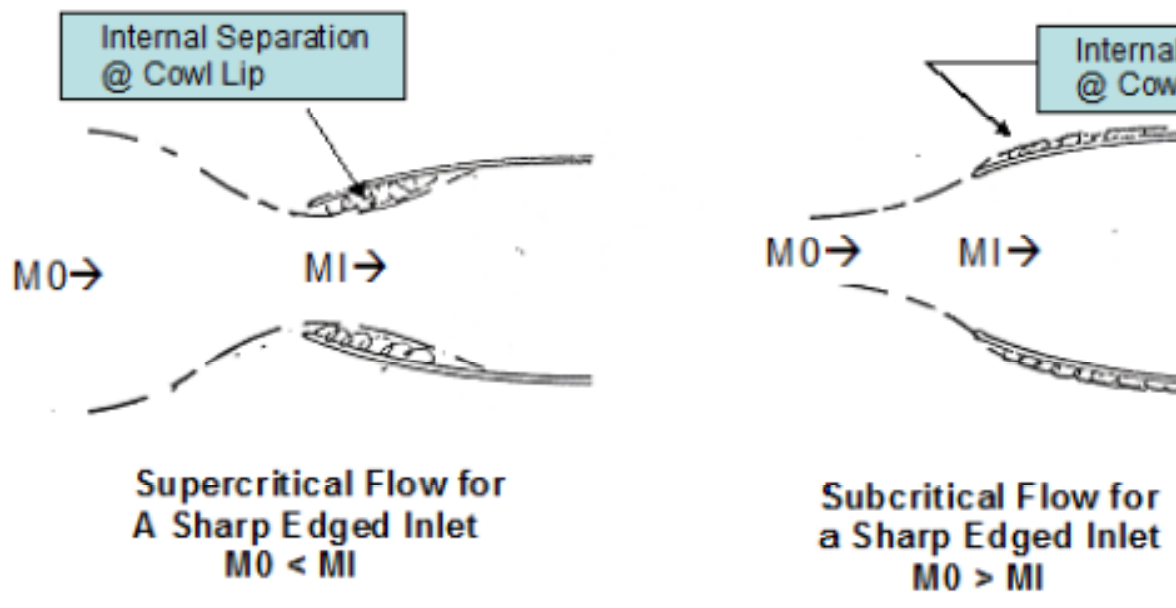

Figure 16. Supercritical and Subcritical Inlet Flow.

In supercritical flow as illustrated on the left, the vehicle's flight speed, $M 0$, is less than the speed of the flow passing through the inlet throat, MI. In this case, the inlet capture area is larger than the throat area and the flow must accelerate into the inlet and round the cowl lip. This leads to internal flow separation and poor pressure recovery at low flight speeds. In subcritical flow, the vehicle's flight speed is greater than the inlet throat speed, indicating that the throat area is greater than the capture area. In this case, the flow does not have to round the cowl lip and internal flow separation is minimized. There is however drag caused by spillage where the flow separates from the outside of the inlet/nacelle in the subcritical case. Spillage drag does not 
affect the performance of the inlet or fan however. For flight speeds above the inlet throat speed, the pressure recovery remains roughly constant.

The function PR2_parabolic allows the user to set the static and subcritical pressure recoveries and flight Mach number where the inlet transitions from supercritical to subcritical. With static and subcritical pressure recoveries of 0.94 and 0.98 , respectively, and a transition Mach number of 0.5, the pressure recovery is estimated as shown in Figure 17.

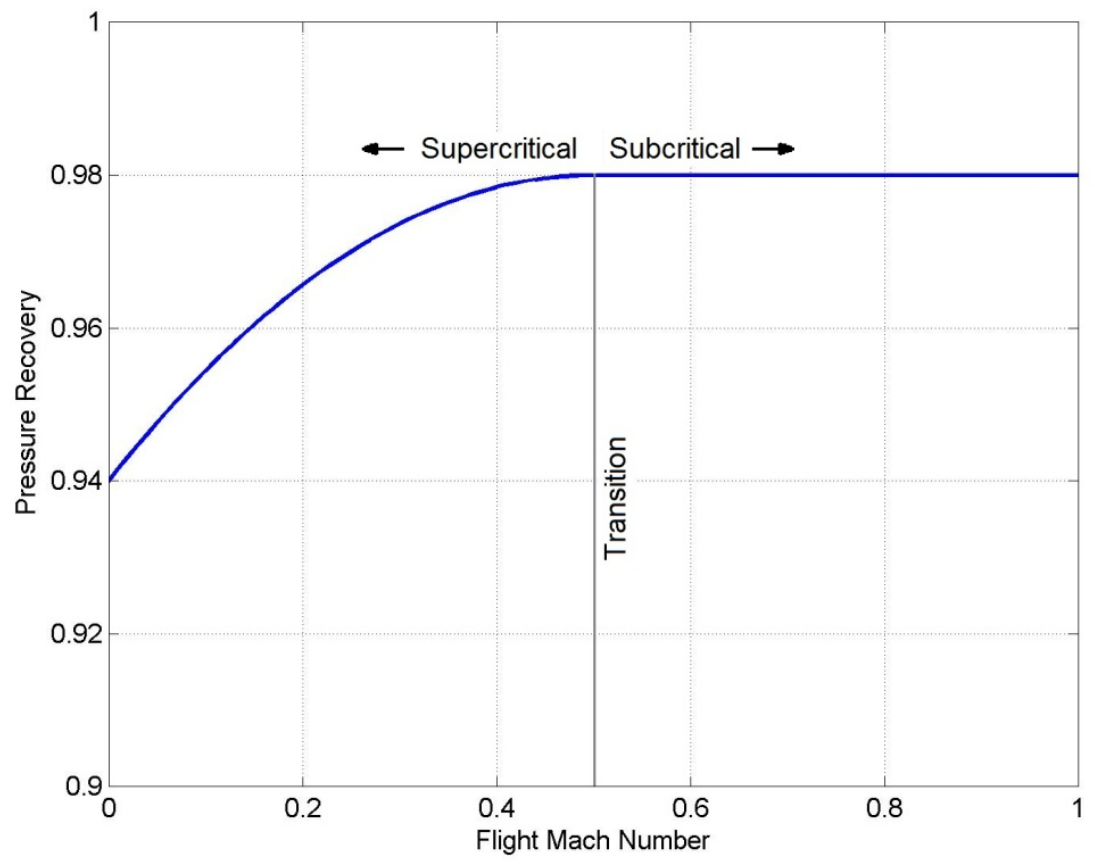

Figure 17. Example of Inlet Pressure Recovery vs. Flight Mach Number.

These values for pressure recovery were estimated in NASA SBIR contract NNX10CC81P for the vehicle shown in Figure 6 which utilized a high aspect ratio square-to-circle cross-section inlet.

The total temperature at the fan face is assumed to be the same as ambient. Using the fan face Mach number (inputted for Mode 1) and the ratio of specific heats (calculated from the thermodynamic properties function), the static temperature and pressure are calculated using the following equations.

$$
\begin{gathered}
T_{2}=T_{a m b} \\
t_{2}=\frac{T_{2}}{\left(1+\frac{\gamma_{2}-1}{2} M_{2}^{2}\right)}
\end{gathered}
$$




$$
p_{2}=\frac{P_{2}}{\left(1+\frac{\gamma_{2}-1}{2} M_{2}^{2}\right)^{\frac{\gamma_{2}}{\gamma_{2}-1}}}
$$

where $M_{2}$ is the fan face Mach number. The total enthalpy in BTU/lb $\mathrm{b}_{\mathrm{m}}$ is calculated using the $4^{\text {th }}$ thermodynamic process function. The static acoustic speed and flow velocity are calculated similarly to equations (4.5.1.3) and (4.5.1.4).

$$
\begin{gathered}
a_{2}=\sqrt{\gamma_{2} R t_{2} g_{c}} \\
V_{2}=M_{2} a_{2}
\end{gathered}
$$

The temperature and pressure ratios are calculated using the total conditions at the fan face. These values are used later to calculate the corrected thrust and mass flow rate.

$$
\begin{aligned}
& \theta=\frac{T_{2}}{T_{s l s}} \\
& \delta=\frac{P_{2}}{P_{s l s}}
\end{aligned}
$$

where $\theta$ and $\delta$ are the temperature and pressure ratios, respectively. The weight flow function at the fan face is also calculated here using the $5^{\text {th }}$ thermodynamic process function. The weight flow function is used later to determine the fan face area.

\section{Station 25 - Stator Entrance}

Because the rotor is the moving component of the fan propulsor, the energy addition to the flow is performed in the rotor alone. This energy is added by means of absolute velocity and pressure, and there is a temperature rise due to the inefficiency of the of the compression process. The total pressure after the rotor is calculated simply using the fan pressure ratio.

$$
P_{25}=P_{2} \pi_{f}
$$

where $\pi_{f}$ is the fan pressure ratio. Thrust producing fans typically have a pressure ratio of 1.1 to 1.6. At the design point, the fan pressure ratio will commonly be inputted by the user, and is then calculated for off-design points. The total temperature after the rotor is calculated using the $11^{\text {th }}$ thermodynamic process function which uses the polytropic efficiency, inlet total temperature, pressure ratio, and fuel-to-air ratio of the fan as inputs. This function also calculates the change in total enthalpy through the rotor and the isentropic efficiency. 
The axial velocity through the fan is assumed to remain constant. In reality, the velocity of the fluid does vary in the axial, radial, and whirl directions, and can be very difficult to predict without a detailed 3D analysis. Figure 18 shows the coordinate system commonly used in axial compressor and fan design.

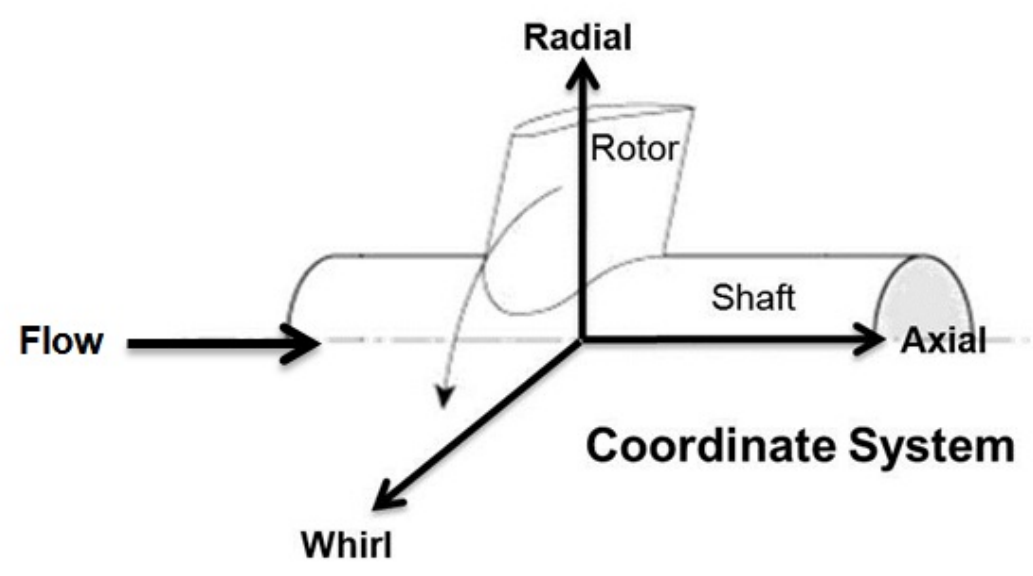

Figure 18. Rotor Coordinate System.

The constant axial velocity assumption however is very common in cycle analysis and has little negative impact on the results. The Mach number after the rotor is calculated using the $16^{\text {th }}$ thermodynamic process function, as shown in the following equations.

$$
\begin{gathered}
V_{25}=V_{2} \\
M_{25}=\frac{V_{25} \sqrt{2}}{\sqrt{2 \gamma_{25} R T_{25} g_{c}-\left(\gamma_{25}-1\right) V_{25}^{2}}}
\end{gathered}
$$

Although the velocity through the fan is assumed to remain constant, the Mach number decreases slightly due to an increase in the total temperature. With Mach number known, the static and total pressure and densities are also calculated. The weight flow function is determined as well and is used later to determine the fan exit area.

\section{Station 3 - Nozzle Entrance}

The purpose of the stator is to straighten the flow by removing the angular momentum, leading to a pressure rise. Although part of the pressure rise is performed in the stator and part in the rotor, the total pressure rise in the fan stage is accounted for in analysis of the rotor. The ratio 
of pressure (or enthalpy) rise in the rotor to that in the stator is called degree of reaction, and is explained in further detail in section 4.7.7.

In this version of HAPSS, the properties of the fluid are assumed to remain constant through the stator. Although in reality there are losses that exist in the stator, this assumption has little effect on the final result. In each mode of the 1D fan analysis, placeholders exist for stator calculations if the user wishes to include them.

\section{Station $6-$ Nozzle Exit}

The purpose of the nozzle is to expand the flow back to near ambient pressure causing the flow to accelerate and thrust to be created. The amount of thrust generated is directly proportional to the difference between the propulsor inlet and exit flow velocities and the amount of fluid being passed through the propulsor.

The total pressure at the nozzle exit is only slightly less than that at the nozzle inlet. Because the nozzle has converging walls (no diverging walls), a favorable pressure gradient exists which prevents flow separation and associated pressure losses. Therefore it can be assumed that the only pressure losses are due to friction with the walls. A common nozzle pressure loss term in cycle analysis is about $1 \%$. The following equation shows the calculation of total pressure at the nozzle exit.

$$
P_{6}=P_{3}\left(1-P R_{6}\right)
$$

where $P R_{6}$ is the pressure drop in the nozzle as a percentage. For the majority of situations, the pressure loss in the nozzle is constant, however the user has the option to create a fan performance function to calculate the pressure loss as a function of other parameters.

The nozzle pressure ratio is calculated next and is simply the ratio of the total pressure at the nozzle exit and the static ambient pressure, as shown in the following equation.

$$
\pi_{6}=\frac{P_{6}}{p_{a m b}}
$$

This equation assumes that the nozzle is optimally expanded, common in cycle analysis. However, to serve as a sanity check, the critical nozzle pressure ratio is also calculated as follows. 


$$
\left(\frac{p^{*}}{P}\right)_{6}=\left(\frac{2}{\gamma_{3}+1}\right)^{\frac{\gamma_{3}}{\gamma_{3}-1}}
$$

If the nozzle pressure ratio is greater than the critical pressure ratio then the flow is said to be choked with a critical/choking mass flow rate $\dot{m}^{*}$. HAPSS issues a warning to the user if the critical pressure ratio in the nozzle is exceeded.

The enthalpy change in the nozzle is calculated using the $2^{\text {nd }}$ thermodynamic process function for a $100 \%$ efficient isentropic expansion to ambient pressure. The nozzle exit velocity is then calculated as follows.

$$
V_{6}=\phi \sqrt{2 \Delta H_{6} g_{c}}
$$

where $\Delta H_{6}$ is the change in total enthalpy in the nozzle and $\Phi$ is the nozzle velocity coefficient. The velocity coefficient is defined as the ratio of the actual exit velocity to the isentropic exit velocity as shown in the following equation.

$$
\phi=\frac{V_{e}}{V_{e}^{\prime}}=\left(\frac{H-h_{e}}{H-h_{e}^{\prime}}\right)^{1 / 2}=\sqrt{\eta_{n}}
$$

where $V_{e}$ is the actual exit velocity, $V_{e}$ 'is the isentropic exit velocity, $H$ is the total enthalpy, $h_{e}$ is the static enthalpy, $h_{e}$ 'is the isentropic static enthalpy, and $\eta_{n}$ is the kinetic energy efficiency. The kinetic energy efficiency usually ranges from 0.930 to 0.996 and reduces the amount of kinetic energy attainable when the fluid is expanded ${ }^{\mathrm{xv}}$.

\section{Fan Performance}

For Mode 1, where the thrust, fan pressure ratio, and fan face Mach number are user inputted, the performance of the fan is calculated first. The mass flow rate in $\mathrm{lb}_{\mathrm{m}} / \mathrm{s}$ through the fan is calculated as follows.

$$
\dot{m}_{f}=\frac{F g_{c}}{V_{6}-V_{a m b}}
$$

where $F$ is the fan thrust in $\mathrm{lb}_{\mathrm{f}}$. The corrected mass flow rate and corrected thrust are calculated next and are useful for relating off-design to sea level static performance.

$$
\begin{gathered}
\dot{m}_{f, c}=\frac{\dot{m} \sqrt{\theta}}{\delta} \\
F_{c}=\frac{F}{\delta}
\end{gathered}
$$


Because the temperature and pressure ratios are calculated using the fan face conditions, the inlet performance is disregarded in the calculation of corrected performance. The amount of power required by the fan, the most important performance parameter from the 1D fan analysis, is calculated as follows.

$$
P w r=\dot{m}_{f} \Delta H_{f}
$$

where $\Delta H_{f}$ is the rise in total enthalpy through the fan. This equation calculates the power in units if $B T U / s$ when the mass flow rate and enthalpy rise are supplied in $1 b_{m} / s$ and $B T U / l b_{m}$. The fan power is then converted to horsepower as follows.

$$
P w r_{h p}=\frac{P w r * J}{I}
$$

where $J$ is the conversion factor of $778.16 \mathrm{ft}-\mathrm{lbf} / \mathrm{BTU}$ and $I$ is the conversion factor 550 (ft$\mathrm{lbf} / \mathrm{s}) / \mathrm{hp}$. The power required of the fan at the design point is the primary sizing parameter for the entire hybrid propulsion system. The final performance value to be calculated in this mode is the volumetric flow rate through the fan in $\mathrm{ft}^{3} / \mathrm{s}$, as shown in the following equation.

$$
Q_{f}=\frac{\dot{m}_{f}}{\frac{\rho_{2}+\rho_{25}}{2}}
$$

where $\rho_{2}$ and $\rho_{25}$ are the static densities at the rotor entrance and rotor exit, respectively. The average rotor density is used because the volumetric flow rate through the rotor is desired. The volumetric flow rate is useful in mode 12 of the $1 \mathrm{D}$ fan performance (see section 4.5.12).

\section{Fan Geometry}

In Mode 1, the fan geometry is calculated once the performance is known. The first parameters that are determined are the fan face and fan exit areas in $\mathrm{ft}^{2}$ which can be calculated using the weight flow functions at these stations. These weight flow functions were determined during the analysis of stations 2 and 25 .

$$
\begin{aligned}
A_{f, 2} & =\frac{\dot{m} \sqrt{T_{2}}}{w f f_{2} P_{2}} \\
A_{f, 25} & =\frac{\dot{m} \sqrt{T_{25}}}{w f f_{25} P_{25}}
\end{aligned}
$$

With the fan face area known, the fan diameter in feet can then be calculated as shown in the following equation. 


$$
D_{f}=2 \sqrt{A_{f, 2}} \sqrt{\frac{-1}{h t r^{2}-1}}(\sqrt{\pi})^{-1}
$$

where $h t r$ is the hub-to-tip ratio. The fan diameter is assumed to be constant from the fan face to the fan exit. Since the fan exit area is always less than that of the fan face, it is also assumed that the fan hub increases in diameter from the fan face to the exit. Fans are typically constructed in this manner as well. The calculation of hub diameter in feet is straightforward.

$$
D_{h}=D_{f} h t r
$$

\section{Fan Speed}

The speed of the fan is the final set of calculations performed in the 1D fan performance analysis. If the on-design point is being analyzed, the fan tip speed in $\mathrm{ft} / \mathrm{s}$ is calculated as follows.

$$
U_{\text {tip }}=M_{\text {tip }}\left(\frac{a_{2}+a_{25}}{2}\right)
$$

where $M_{t i p}$ is the tip Mach number inputted by the user, and $a_{2}$ and $a_{25}$ are the static speeds of sound at the fan face and fan exit, respectively. The average speed of sound is used even though it only increases about $5 \%$ through the fan. The rotational speed of the fan is calculated next in revolutions per minute (RPM).

$$
N=\frac{60 U_{t i p}}{\pi D_{f}}
$$

Next, the affinity laws of the fan are considered which are useful for predicting a fan's performance at an off-design condition. In HAPSS, fan affinity laws are utilized to estimate the fan speed at off-design conditions. During the on-design analysis, the fan power and flow coefficients are calculated as follows.

$$
\begin{gathered}
K_{p}=\frac{P w r_{h p} I}{\left(\frac{\rho_{2}-\rho_{25}}{2}\right)\left(\frac{N}{60}\right)^{3} D_{f}^{5}} \\
K_{f}=\frac{Q}{N D_{f}^{3}}
\end{gathered}
$$

where $K_{p}$ and $K_{f}$ are the power and flow coefficients, respectively. For fans with constant geometry and small changes in speed and efficiency, it can be assumed that these coefficients remain constant. For the off-design analysis, the fan rotational speed, tip speed, and tip Mach number can be calculated using the power coefficient from the on-design condition. The flow coefficient is used for mode 12 of the 1D fan performance analysis (see section 4.5.12). 
The final parameter calculated during mode 1 is the fan torque in $\mathrm{ft}-\mathrm{lb}_{\mathrm{f}}$ as shown in the following equation.

$$
T=\frac{33000 P w r_{h p}}{2 \pi N}(4.5 .1 .37)
$$

The torque required by the fan is a very important design parameter as it is the primary sizing metric for electric motors.

\subsubsection{Mode 2}

This mode of the 1D performance analysis requires the user to input fan pressure ratio, fan face Mach number, and the available power, and outputs fan diameter, thrust, and mass flow rate. This mode would typically be used for sizing the fan at the on-design condition when the amount of available power is known, but the thrust is not. This mode is nearly identical to mode 1 , but calculates the fan performance in a slightly different manner.

In this mode, the user supplies fan power in horsepower. The power is first converted to BTU/s as shown in the following equation.

$$
P w r=\frac{P w r_{h p I}}{J}
$$

The mass flow rate through the fan is calculated using the fan power and enthalpy rise in the fan as shown here followed by the calculation of fan thrust in $\mathrm{lb}_{\mathrm{f}}$.

$$
\begin{gathered}
\dot{m}_{f}=\frac{P w r}{\Delta h_{f}} \\
F=\frac{\dot{m}_{f}\left(v_{6}-v_{a m b}\right)}{g_{c}}
\end{gathered}
$$

The corrected mass flow rate and thrust are calculated in the same manner as in mode 1. The determination of fan geometry is also identical to mode 1.

\subsubsection{Mode 5}

This mode of the 1D performance analysis requires the user to input fan pressure ratio, mass flow rate, and fan face Mach number, and outputs fan diameter, thrust, and required power. This mode was created for validation purposes against published experimental data. This mode is also very similar to previous modes. The fan thrust is calculated as in mode 2 (equation 4.5.2.3), and the fan power is calculated as in mode 1 (equation 4.5.1.26). 


\subsubsection{Mode 8}

This mode is the last of the 1D fan performance modes that can be solved linearly (does not require an iterative solution). This mode requires to user to input fan pressure ratio, fan diameter, and fan face Mach number, and outputs thrust, mass flow rate, and required power. Since fan diameter is inputted, the fan geometry is calculated first including the fan face area and hub diameter.

Next, the mass flow rate is calculated using the fan face area and the weight flow function at station 2 determined earlier. This calculation is shown here.

$$
\dot{m}_{f}=\frac{A_{f, 2} w f f_{2} P_{2}}{T_{2}}
$$

The thrust produced by the fan is then calculated as in equation 4.5.2.3, and the power is calculated as in equation 4.5.1.26.

\subsubsection{Mode 3}

This mode of the 1D performance analysis requires the user to input fan pressure ratio, fan diameter, and power, and outputs thrust, mass flow rate, and fan face Mach number. This mode is very useful for off-design conditions where the fan pressure ratio is known.

Because the fan face Mach number must be known early in the analysis of the fan performance, this mode uses an iterative approach. The parameter that is varied is the fan face Mach number and the parameter that is targeted is the fan diameter. In other words, this mode varies the fan face Mach number until the desired fan diameter is achieved within a specified tolerance. MATLAB's minimizer fminbnd is used to find the fan face Mach number that minimizes the error in the fan diameter. This function is part of MATLAB's optimization toolbox.

In the mode 3 script, the target fan diameter is set first. Then, the user specified tolerances for fminbnd are set. This minimizer uses two tolerances; TolX being the tolerance on the change in the guessed variable (fan face Mach number in this mode), and TolFun being the tolerance on the objective function's output (fan diameter error in this mode). The upper and lower bounds on the fan face Mach number are also defined; 0 and 1, respectively, suitable for 
most situations. Next, the optimizer is run by being passed an objective function, the lower and upper bounds, and the settings for tolerance.

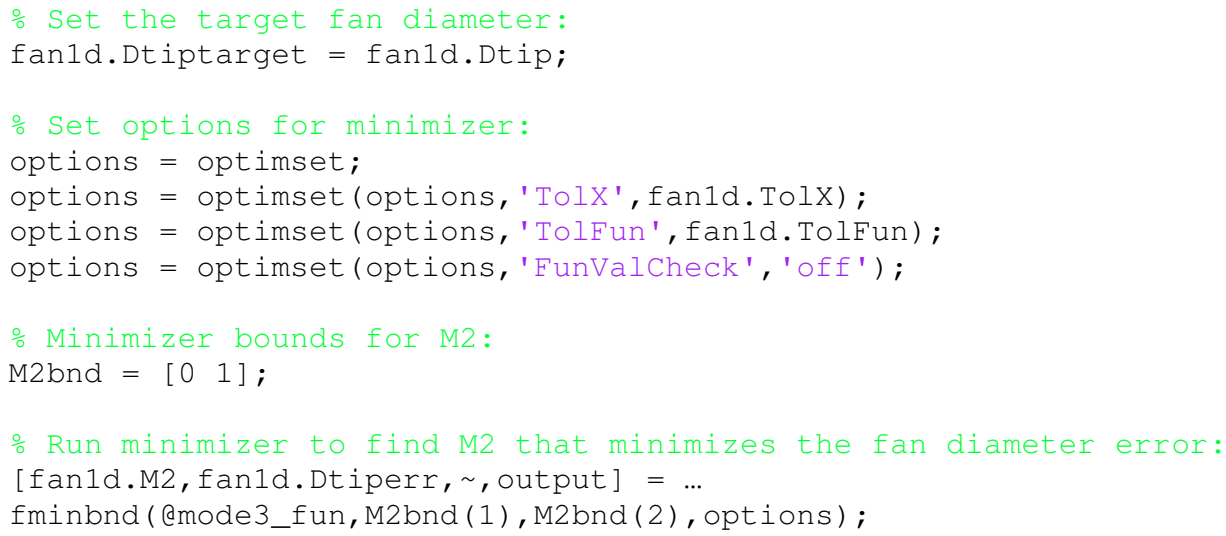

The objective function is a script that uses the guess for fan face Mach number and outputs the error in fan diameter. For mode 3, the objective function runs mode 2 with the guessed fan face Mach number, fixed fan pressure ratio, and fixed power. Mode 2 then outputs the fan diameter and compares it to the desired fan diameter of mode 3, creating an error. The function fminbnd continuous to guess new fan face Mach numbers until the error in fan diameter (TolFun) or the change in the fan face Mach number (TolX) are below tolerances. The mode 3 objective function is shown here.

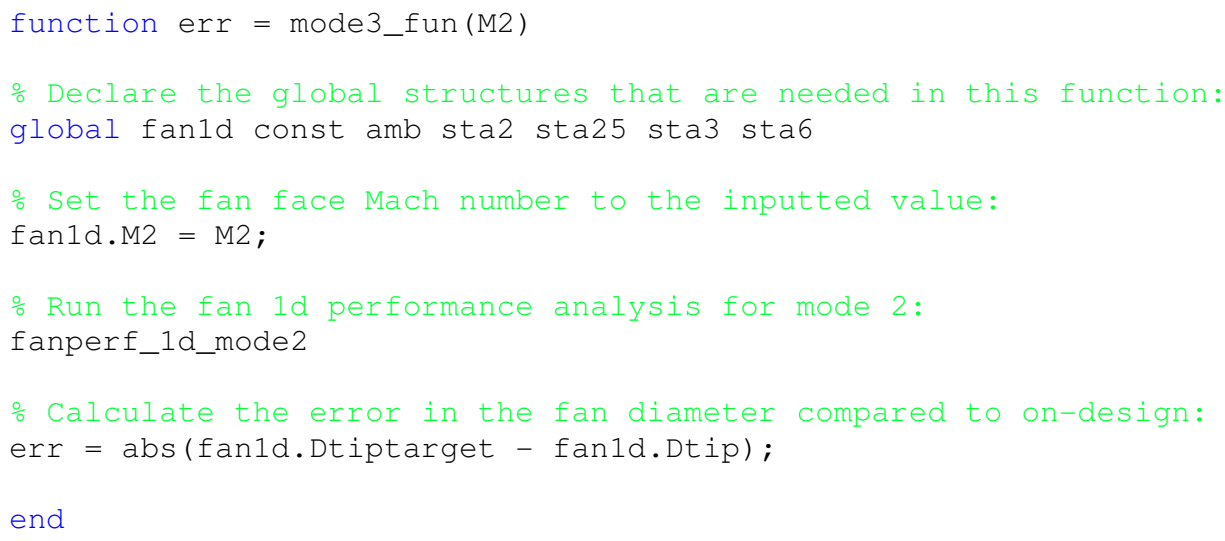

With tolerances TolX and TolFun set at $10^{-5}$ and $10^{-9}$, respectively, the minimizer fminbnd requires 20-30 iterations to find the fan face Mach number that satisfies the fan diameter requirement. The mode 3 script then checks to ensure the minimizer converged within the tolerances TolFun (as the minimizer can sometimes achieve TolX before TolFun). If convergence 
was not achieved, MATLAB displays a warning in the command window and information on the flight condition that was being analyzed. This checking is shown here.

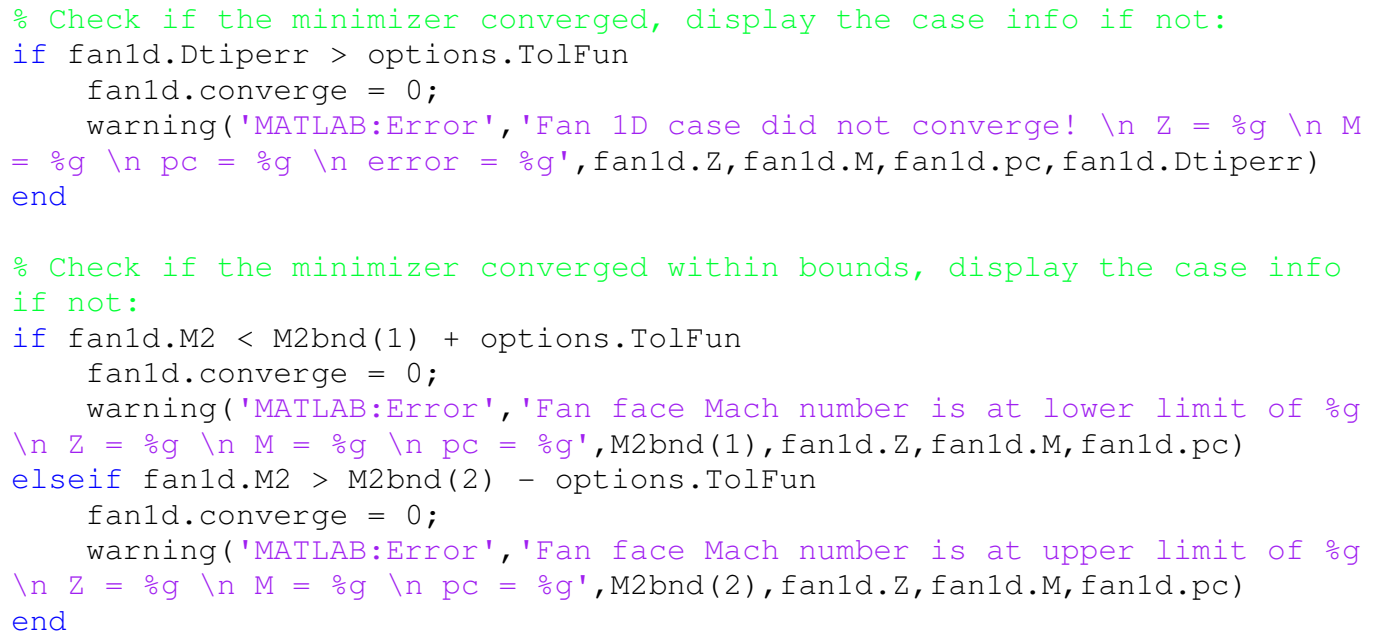

\subsubsection{Mode 4}

This mode of the 1D performance analysis is a unique analysis that requires the user to input only the required thrust and fan face Mach number, and outputs the fan pressure ratio, fan diameter, and power, and mass flow rate. Unlike the modes that have been discussed, this mode only requires two inputs from the user, and uses optimization to find a fan pressure ratio that minimizes the power required by the fan. This is the only mode that uses optimization to minimize one of the output variables. Because the power required by the fan is the foremost driver for the sizing of the hybrid propulsion system, this mode is very useful at the on-design condition.

An example was created to show the how the fan power changes with pressure ratio. In this example, the fan design point is an altitude of $30,000 \mathrm{ft}$ and a flight Mach number of 0.7 . The fan must produce $100 \mathrm{Ib}_{\mathrm{f}}$ of thrust with a fan face Mach number of 0.6 , a polytropic efficiency of $95 \%$, and an inlet pressure recovery of $98 \%$. The fan power and diameter versus pressure ratio for this example is shown in Figure 19. 


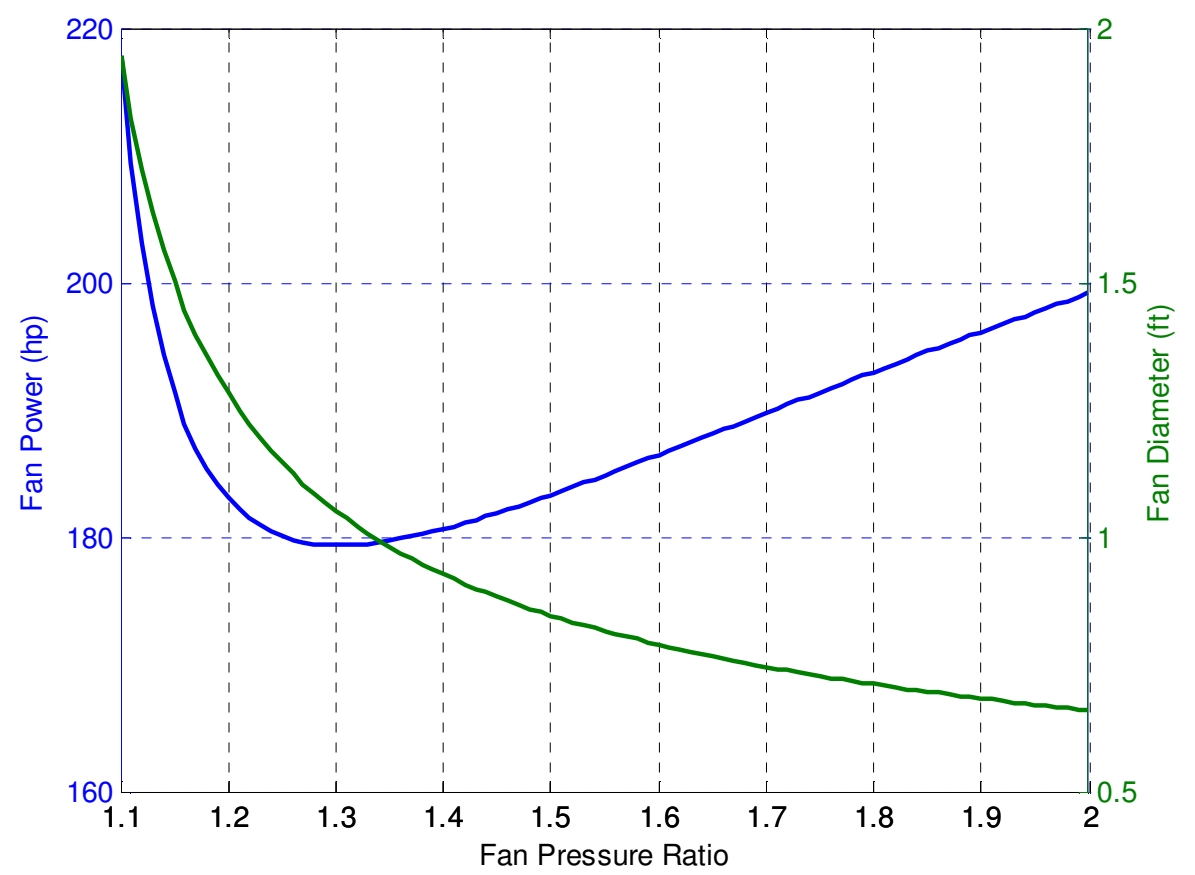

Figure 19. Example of Fan Power and Diameter vs. Fan Pressure Ratio.

In this figure, the blue and green lines show the fan power required and the fan diameter, respectively. For this example, the minimum power occurs at a pressure ratio of about 1.31. Generally speaking, it is also desirable to reduce fan diameter, however the fan diameter decreases monotonically with fan pressure ratio never reaching a true minimum. The flight speed has a significant influence on the optimal pressure ratio since the fan must generate an adequate change in velocity to produce the required thrust. Inlet pressure recovery, nozzle pressure loss, and the nozzle velocity coefficient also significantly affect the optimal pressure ratio. Provided that losses exist in the inlet and nozzle the minimum power required will always occur at a pressure ratio greater than one.

This mode is similar to mode 3 in operation, however the fan pressure ratio is varied instead of the fan face Mach number, and the minimized parameter is the fan power instead of the error in fan diameter. Mode 4 also uses mode 1 during its analysis. fminbnd is utilized in this mode as well with practical lower and upper bounds for the fan pressure ratio at set at 1 and 10 , respectively. This mode also completes in approximately 20-30 iterations. 


\subsubsection{Mode 6}

This mode of the 1D performance analysis requires the user to input fan diameter, power, and fan face Mach number, and outputs fan pressure ratio, thrust, and mass flow rate. This mode would be most useful for off-design analyses where the fan and inlet geometry and power available are known, but the pressure ratio is not.

Because the fan pressure ratio must be known early in the analysis of the fan performance, this mode uses an iterative approach. The parameter that is varied is the fan pressure ratio and the parameter that is targeted is the fan diameter. In other words, this mode varies the fan pressure ratio until the desired fan diameter is achieved within a specified tolerance. Similar to mode 3 and 4, MATLAB's minimizer fminbnd is used to find the fan pressure ratio that minimizes the error in the fan diameter. This mode however uses mode 2 instead of mode 1 in the iterative approach.

\subsection{8 $\quad$ Mode 7}

This mode of the 1D performance analysis requires the user to input thrust, power, and fan face Mach number, and outputs fan pressure ratio, mass flow rate, and fan diameter. This mode would be most useful for on-design analyses where the required thrust and power available are known, but the pressure ratio and fan geometry not.

Similar to mode 6 , this mode also varies the fan pressure ratio but attempts to minimize the error in fan thrust within the specified tolerance. This mode also uses mode 2 in the iterative approach.

\subsubsection{Mode 9}

This mode of the 1D performance analysis requires the user to input thrust, fan diameter, and fan face Mach number, and outputs fan pressure ratio, mass flow rate, and required power. This mode would be most useful for off-design analyses where the required thrust and fan geometry are known, but the pressure ratio and required power are not.

This mode too varies the fan pressure ratio but to minimize the error in the fan diameter. This mode uses mode 1 in the iterative approach. 


\subsubsection{Mode 10}

This mode of the 1D performance analysis requires the user to input thrust, fan diameter, and power, and outputs fan pressure ratio, mass flow rate, and fan face Mach number. This mode is unique as it requires two separate minimization problems to be performed since both pressure ratio and fan face Mach number are required parameters early in the fan analysis.

Interestingly however, these two parameters can be solved for independently of one another. First, the fan pressure ratio is solved for by minimizing the error in the fan thrust. The fan face Mach number in this minimization problem has no effect on the fan thrust. Next, the fan face Mach number is solved for by minimizing the error in fan diameter. This mode uses only mode 2 in both of the iterative approaches. Since both minimization problems can be solved independently, this mode only requires $40-60$ total iterations.

\subsubsection{Mode 11}

This mode of the $1 \mathrm{D}$ performance analysis requires the user to input fan pressure ratio, thrust, and fan diameter, and outputs mass flow rate, required power, and fan face Mach number. This mode varies the fan face Mach number to minimize the error in the fan diameter using mode 1.

\subsubsection{Mode 12}

This mode of the 1D performance analysis requires the user to input the available power and fan diameter, and outputs the fan pressure ratio, thrust, mass flow rate, and fan face Mach number. This mode can only be used for off-design analysis where the fan geometry and available power are known. Unlike the previous modes, mode 12 only requires the user to input two of the six main design variables making it the most versatile of the modes.

This mode requires two minimization problems to occur, one embedded in the other. The inner minimization problem varies fan pressure ratio to minimize the error in fan diameter, identical to mode 6 . The outer minimization problem varies the fan face Mach number to minimize the error in the mass flow rate. The target mass flow rate is calculated during the inner 
minimization problem. The iterated mass flow rate in this case is calculated using the fan flow coefficient calculated during the on-design analysis as shown in the following equations.

$$
\begin{gathered}
Q_{f}=K_{f} N D_{f}^{3} \\
\dot{m}_{f}=Q_{f} \frac{\rho_{2}+\rho_{25}}{2}
\end{gathered}
$$

Because this mode has two minimization problems, one inside the other, it requires many more iterations to complete, about $1500-4000$ in most cases.

\subsubsection{Validation}

The 1D performance analysis was validated using several sources. The first source was a detailed turbofan cycle analysis code written by Mark Waters. Mode 1 of the $1 \mathrm{D}$ fan analysis in HAPSS will be used for this validation since the inputs to Mark Waters' code are fan pressure ratio, fan face Mach number, and required thrust. Table 4 shows the inputs that were used for both codes, while Table 5 shows the outputs from both codes and percent differences.

Table 4. 1D Fan Performance Validation Inputs.

\begin{tabular}{|l|c|c|}
\hline \multicolumn{1}{|c|}{ Input } & Units & Value \\
\hline Design altitude & $\mathrm{ft}$ & 30000 \\
\hline Design flight Mach number & - & 0.65 \\
\hline Required thrust & $\mathrm{Ib}_{\mathrm{f}}$ & 450 \\
\hline Fan pressure ratio & - & 1.35 \\
\hline Fan face Mach number & - & 0.62 \\
\hline Hub-to-tip ratio & - & 0.3 \\
\hline Fan polytropic efficiency & $\%$ & 95 \\
\hline Nozzle duct velocity drop & $\%$ & $1 \%$ \\
\hline Nozzle duct pressure drop & $\%$ & $1 \%$ \\
\hline Inlet pressure recovery & $\%$ & 99 \\
\hline
\end{tabular}

Table 5. 1D Fan Performance Validation Outputs.

\begin{tabular}{|l|c|c|c|c|}
\hline \multicolumn{1}{|c|}{ Output } & Units & $\begin{array}{c}\text { Waters' } \\
\text { Code }\end{array}$ & HAPSS & Difference \\
\hline Mass flow rate & $\mathrm{lb}_{\mathrm{m}} / \mathrm{s}$ & 52.05 & 51.91 & $-0.27 \%$ \\
\hline Power required & $\mathrm{hp}$ & 744.26 & 743.63 & $-0.08 \%$ \\
\hline Exit velocity & $\mathrm{ft} / \mathrm{s}$ & 924.49 & 925.89 & $0.15 \%$ \\
\hline Fan diameter & $\mathrm{ft}$ & 2.03 & 2.02 & $-0.49 \%$ \\
\hline Fan exit enthalpy & $\mathrm{BTU} / \mathrm{b}_{\mathrm{m}}$ & 116.61 & 116.87 & $0.22 \%$ \\
\hline Fan exit temperature & ${ }^{\circ} \mathrm{R}$ & 487.91 & 488.97 & $0.22 \%$ \\
\hline Fan exit pressure & $\mathrm{lb} / \mathrm{in}^{2}$ & 7.68 & 7.75 & $0.91 \%$ \\
\hline
\end{tabular}


It can be seen from the table that the both HAPSS and Mark Waters' code produce very similar results. This is a result of both codes using analyses based on thermodynamics. Error between the two codes could be a result of rounding, differences in calculated gas properties, and the assumptions that were made.

The other two validation sources were actual fan performance data for a General Electric CF6 turbofan engine and data produced by Numerical Propulsion System Synthesis (NPSS), a NASA cycle analysis program. Unfortunately, the CF6 data was lost during the course of this thesis and a working license for NPSS has yet to be attained to reproduce the validation. The pertinent errors for these validations were under $5 \%$ however. An initial goal for future iterations of HAPSS will be to reproduce the NPSS validation.

\subsection{Fan Weight Analysis}

In the on-design analysis, the weight of the fan assembly is estimated. The fan weight script in HAPSS estimates this weight using fan geometry and performance calculated during the 1D analysis in addition to numerous inputs supplied by the user. The methodology for this analysis came from the NASA WATE-1 (Weight Analysis of Turbine Engines) program as discussed in NASA CR135170 ${ }^{\text {xvi }}$ and NASA CR168049xvii . The goal of the WATE program was to estimate turbine engine weight with $\pm 10 \%$ accuracy, however in practice is accurate down to $\pm 5 \%$. The program was developed using data from 29 turbine engines including military and commercial turbofans and turbojets.

Although the WATE program estimates total engine weight, only the methodology for fans and compressors was adopted for use in HAPSS. Furthermore, this method is only meant to provide a mere approximation of the fan weight. A single-stage motor-driven fan assembly would different greatly in design than fans for use on turbine engines. Figure 20 shows a typical fan/compressor stage analyzed by WATE. 


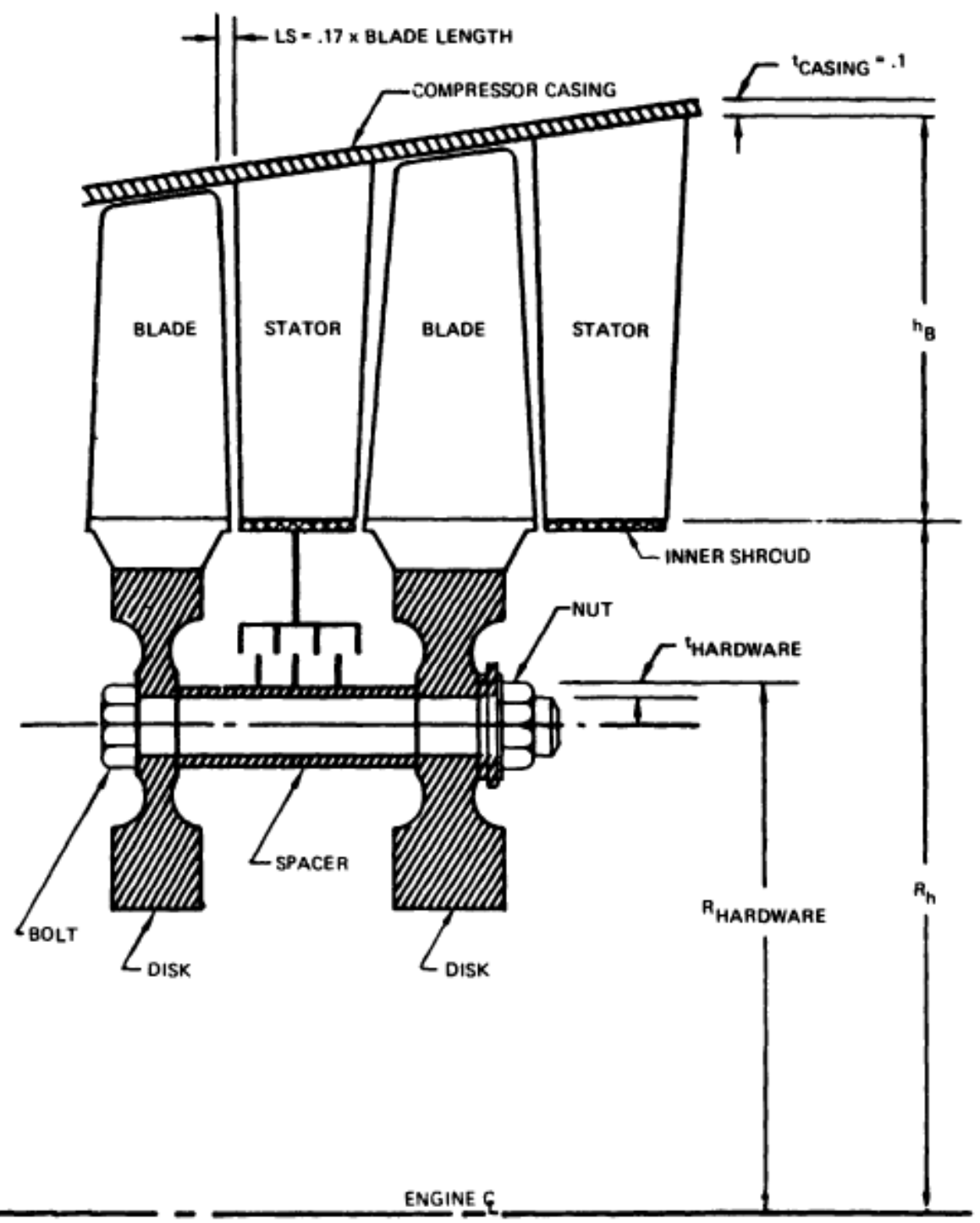

Figure 20. Fan/Compressor Stage Diagram ${ }^{\mathrm{xvii}}$.

This weight estimation method requires the user to input many parameters which could be too detailed for early analyses. With this is mind, the HAPSS code uses reasonable default values if any of the inputs are left undefined. The weight estimation uses the following inputs.

- Rotor and stator, disc, hardware, casing, and shaft material densities

- Shaft yield stress and factor of safety

- Fan case thickness

- Rotor and stator blade volume factor

- Rotor and stator blade aspect ratio and taper ratio

- Rotor and stator solidity 
- Rotor overspeed factor

- Fan mounting frame count and type

- Miscellaneous inputs from the 1D fan analysis

The fan weight script first checks all of the inputs to ensure they are within limits. If an input is not defined then a default value is given.

\subsubsection{Rotor/Stator Weight}

The weight of the rotor and stator are calculated identically and in this iteration it is assumed that the mean radii of the two are equal. The geometry used during this analysis is shown in Figure 21.
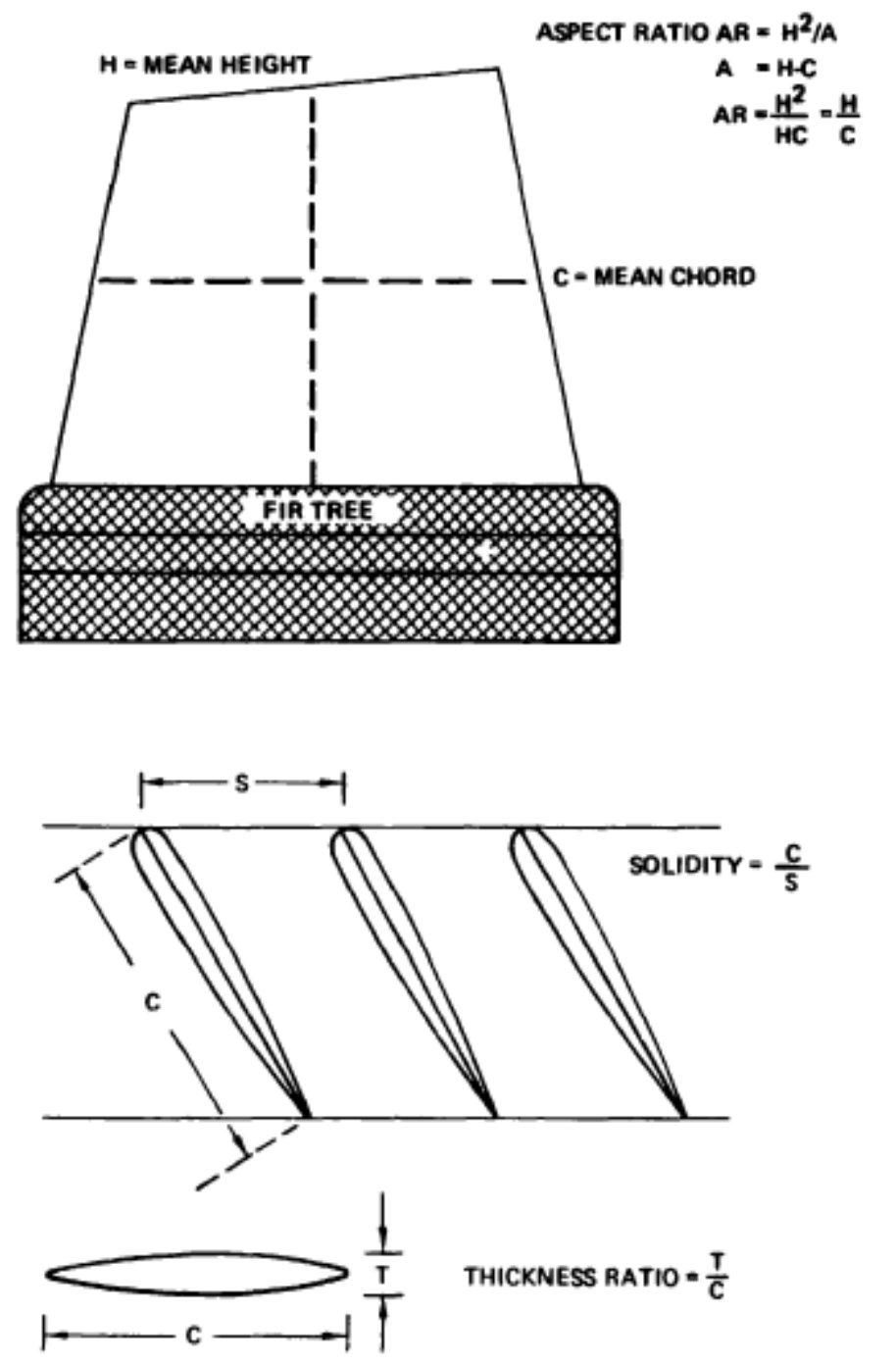

Figure 21. Blade Geometry for Fan Weight Estimation ${ }^{\text {xvii }}$. 
The first step in the fan weight estimation is the calculation of the mean blade height. In this iteration of HAPSS it is assumed that the height of the blades is constant.

$$
h_{b}=\frac{D_{f}}{2}-\frac{D_{h}}{2}
$$

where $h_{b}$ is the mean blade height and $D_{f}$ and $D_{h}$ are the fan tip and hub diameters calculated in the 1D fan analysis, all in feet. For fan blades the volume factor was found to be approximately 0.055 and 0.012 for compressor blades. For blades with a hub-to-tip ratio greater than 0.8 , the volume factor can be estimated.

$$
K_{v o l}=0.120+0.04(h t r * 0.8)
$$

where $h t r$ is the hub-to-tip ratio. The volume of a single blade in $\mathrm{ft}^{3}$ and the mean blade chord in feet are then calculated as follows.

$$
\begin{gathered}
\forall_{b}=\frac{K_{v o l} h_{b}^{3}}{A R^{2}} \\
c_{b}=\frac{h_{b}^{3}}{A R}
\end{gathered}
$$

where $A R$ is the blade aspect ratio. In order to calculate the total weight of the rotor and stator, and number of blades for each must be calculated as follows.

$$
N_{b}=\frac{\pi D_{f} \sigma A R}{h_{b}^{3}}
$$

where $\sigma$ is the solidity, defined as the blade chord divided by the blade spacing (see Figure 21). Finally the rotor/stator weight can be calculated in pounds.

$$
W_{\text {rotor } / \text { stator }}=N_{b} \rho_{b} \forall_{b}
$$

where $\rho_{b}$ is the density of the blade material in $\mathrm{lb} / \mathrm{ft}^{3}$. The calculation of the rotor/stator length is shown here in feet.

$$
L_{\text {rotor } / \text { stator }}=h_{b} A R
$$

\subsubsection{Rotor Disc Weight}

The rotor disc holds the compressor/fan blades in place and endures an incredible amount of stress during operation. In order to withstand these stresses rotor discs tend to be heavy and contribute significantly to the overall weight of the assembly. First, the blade-pull stress on the disc is calculated in $\mathrm{lb} / \mathrm{ft}^{2}$. 


$$
\sigma_{\text {disc }}=\frac{\rho_{b} U^{2}}{g_{c} T R}\left[\frac{1-h t r^{2}}{2}+\frac{T R-1}{12}(1-h t r)(1+3 h t r)\right]
$$

where $T R$ is the taper ratio of the rotor blade and $U$ is the blade tip speed in $\mathrm{ft} / \mathrm{s}$. This fan weight analysis uses the tip speed from the 1D fan on-design analysis multiplied by the overspeed factor. Next, the relative disc loading is calculated in $\mathrm{lb} / \mathrm{ft}$.

$$
\sigma_{\text {disc,rel }}=\frac{\sigma_{\text {disc } D_{h}}}{2 E 5}
$$

Using the relative disc loading, the relative disc thickness can be estimated using a figure of historical data shown in Figure 22.

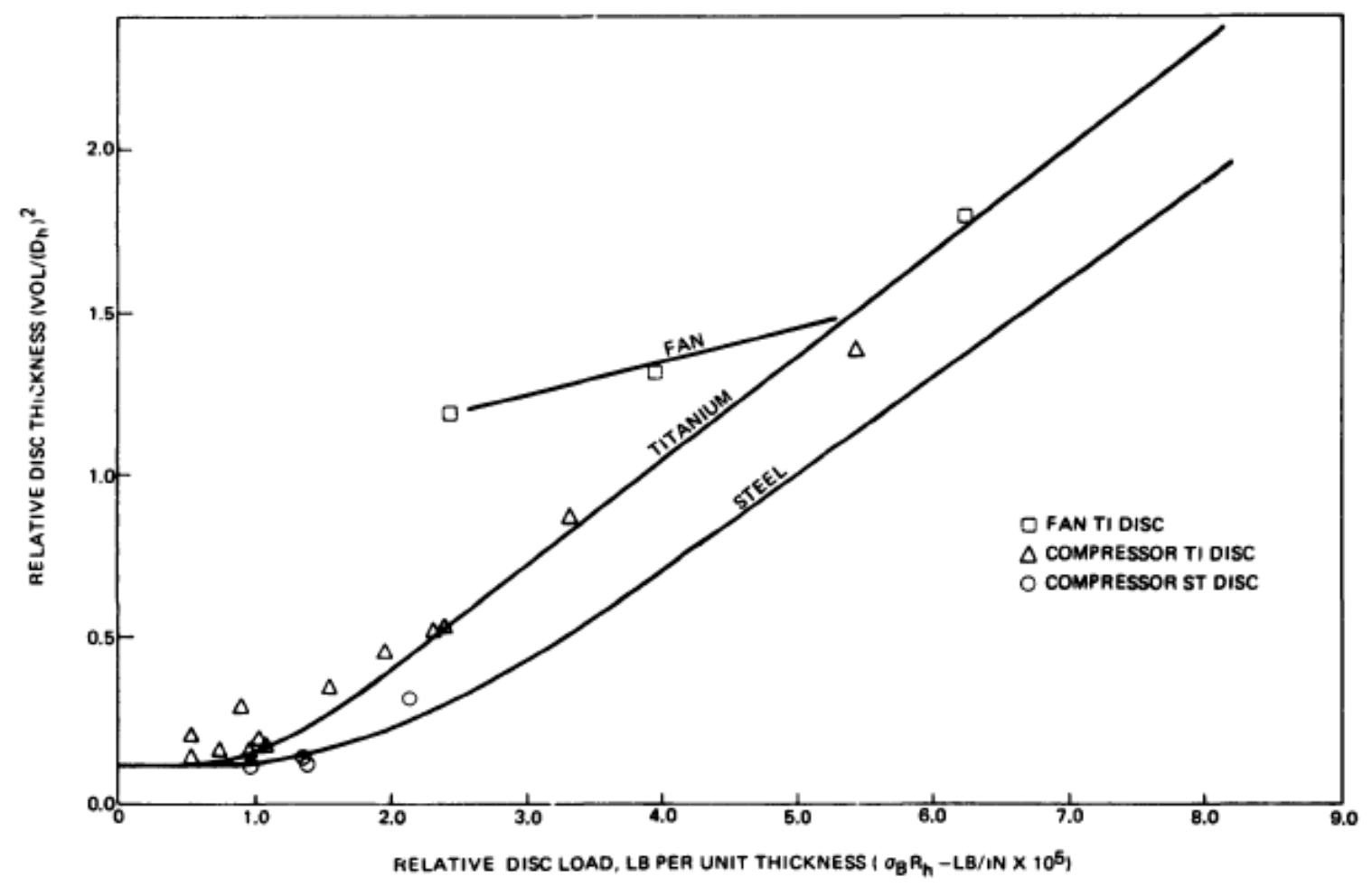

Figure 22. Relative Disc Thickness vs. Relative Disc Loading ${ }^{x v i}$.

These three curves were transformed into curve-fit equations such that they could be utilized in the HAPSS code. When the hub-to-tip ratio is lower than 0.5 , likely indicating a fan, the titanium fan disc line is used. When the hub-to-tip ratio is greater than 0.5 , likely indicating a compressor, and the disc density is less than $385 \mathrm{lb} / \mathrm{ft}^{3}$, the titanium compressor disc line is used. And for greater densities the steel compressor disc line is used. A dividing density of $385 \mathrm{lb} / \mathrm{ft}^{3}$ was chosen since it is approximately halfway between titanium and steel. The rotor disc volume and weight are then calculated as follows. 


$$
\begin{aligned}
& V_{\text {disc }}=t_{\text {disc,rel }} D_{h}^{2} \\
& W_{\text {disc }}=V_{\text {disc }} \rho_{\text {disc }}
\end{aligned}
$$

\subsubsection{Hardware Weight}

The hardware weight is an estimation of the bolts, nuts, washers, and other miscellaneous fasteners that hold together the fan stage. The weight of the spacers (see Figure $20)$ is also included in this calculation.

$$
W_{h w}=2 \pi\left(0.75 \frac{D_{h}}{2}\right) 0.006226\left(1.17 L_{\text {rotor }}+L_{\text {stator }}\right) \rho_{h w}
$$

In this equation, the spacers are assumed to be $0.006226 \mathrm{ft}(0.075 \mathrm{in})$ thick and are located at $75 \%$ of the radius of the hub.

\subsubsection{Shaft Weight}

The weight of the shaft was calculated using a different method than prescribed in the WATE documentation. The WATE method requires knowledge of the shaft diameters and is for concentric multiple-spool shafts. The propulsor fans in a hybrid aircraft would likely not use concentric shafts, but rather simple solid or hollow shafts. The method used in HAPSS is for simple solid shafts. First, the maximum allowable shear stress in $\mathrm{lb}_{\mathrm{ft}}{ }^{2}$ of the shaft is calculated.

$$
\tau_{\text {max }}=\tau_{\text {shaft }} F_{\text {oshaft }}
$$

where $T_{\text {shaft }}$ is the shearing stress of the shaft material in $\mathrm{lb} / \mathrm{ft}^{2}$ and $F o S_{\text {shaft }}$ is the desired factory of safety for the shaft. Using this maximum stress, the diameter of the shaft is calculated.

$$
D_{\text {shaft }}=2\left[\left(\frac{2 T}{\pi \tau_{\max }}\right)^{\frac{1}{3}}\right]
$$

where $T$ is the torque of the fan in $\mathrm{ft}^{-} \mathrm{lb}_{\mathrm{f}}$ calculated during the $1 \mathrm{D}$ performance analysis. Finally, the weight of the shaft is calculated using the material density and the stage length.

$$
W_{\text {shaft }}=\pi\left(\frac{D_{\text {shaft }}}{2}\right)^{2}\left(1.17 L_{\text {rotor }}+L_{\text {stator }}\right) \rho_{\text {shaft }}
$$




\subsubsection{Case Weight}

The weight of the casing that encloses the fan assembly contributes significantly to the overall assembly weight. The WATE method assumes a case thickness of $0.254 \mathrm{~cm}$, however in HAPSS the user can specify the case thickness. This calculation is as follows.

$$
W_{\text {case }}=\pi D_{f}\left(1.17 L_{\text {rotor }}+L_{\text {stator }}\right) \rho_{\text {case }} t_{\text {case }}
$$

where $t_{\text {case }}$ is the thickness of the case in feet, inputted by the user.

\subsubsection{Frame Weight}

The final contribution to the fan assembly weight is the frame. The frame supports the fan assembly, typically at both ends and sometimes near the center. According to Boeing and the WATE manual, the frame weight correlated well with the frame's projected area. There were four frame types that were considered for the WATE method; single-bearing with and without power takeoff, turbine exit, and intermediate. Figure 23 shows where these frame types might be located on a turbine engine. 

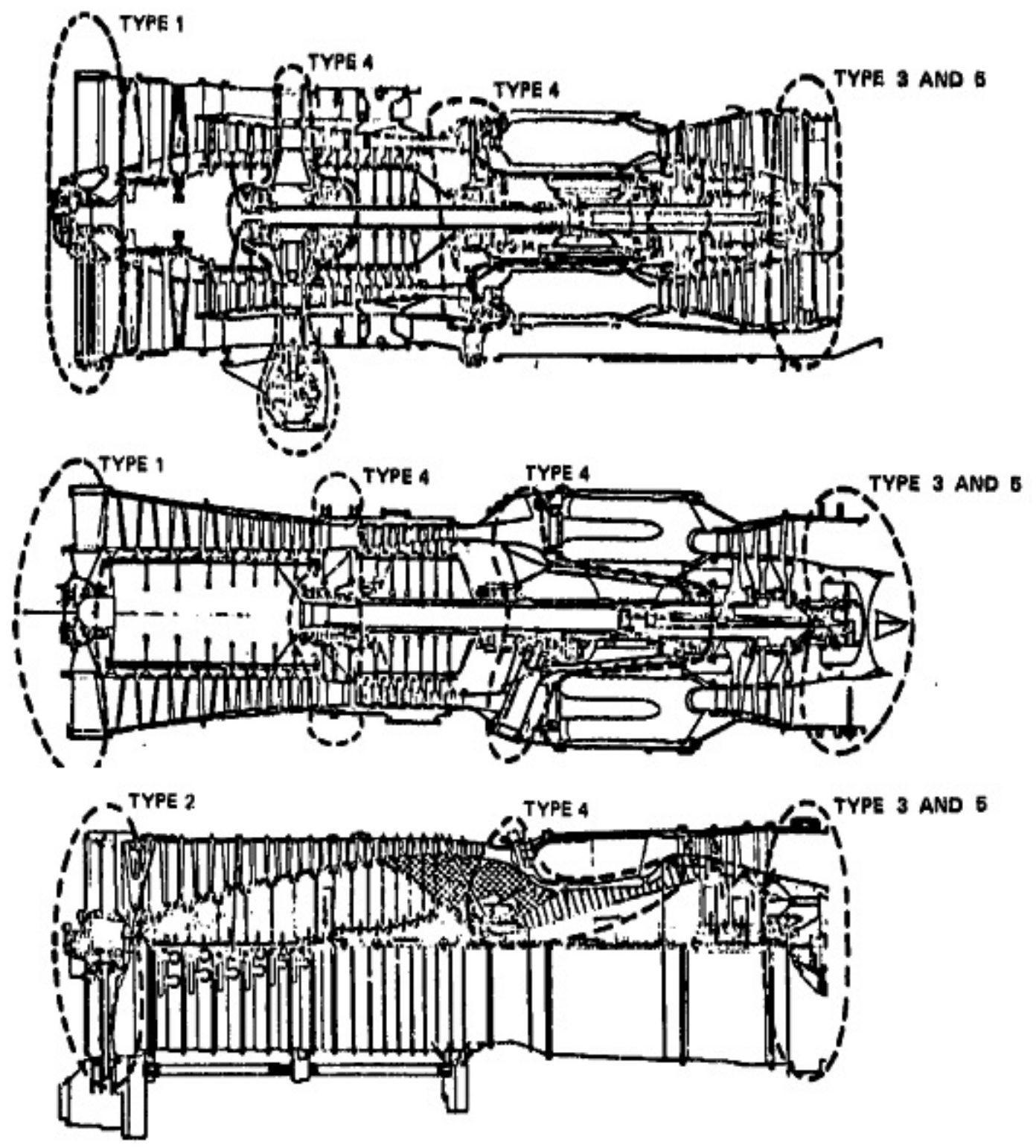

Figure 23. Engine Frame Types ${ }^{\text {xvii }}$.

In this figure, Type 1 is a single-bearing frame without power takeoff, Type 2 is a singlebearing frame with power takeoff, Type 3 is a turbine frame, and Type 4 is an intermediate 2bearing or burner frame. Figure 24 shows the estimated weight for these four types of frames. 


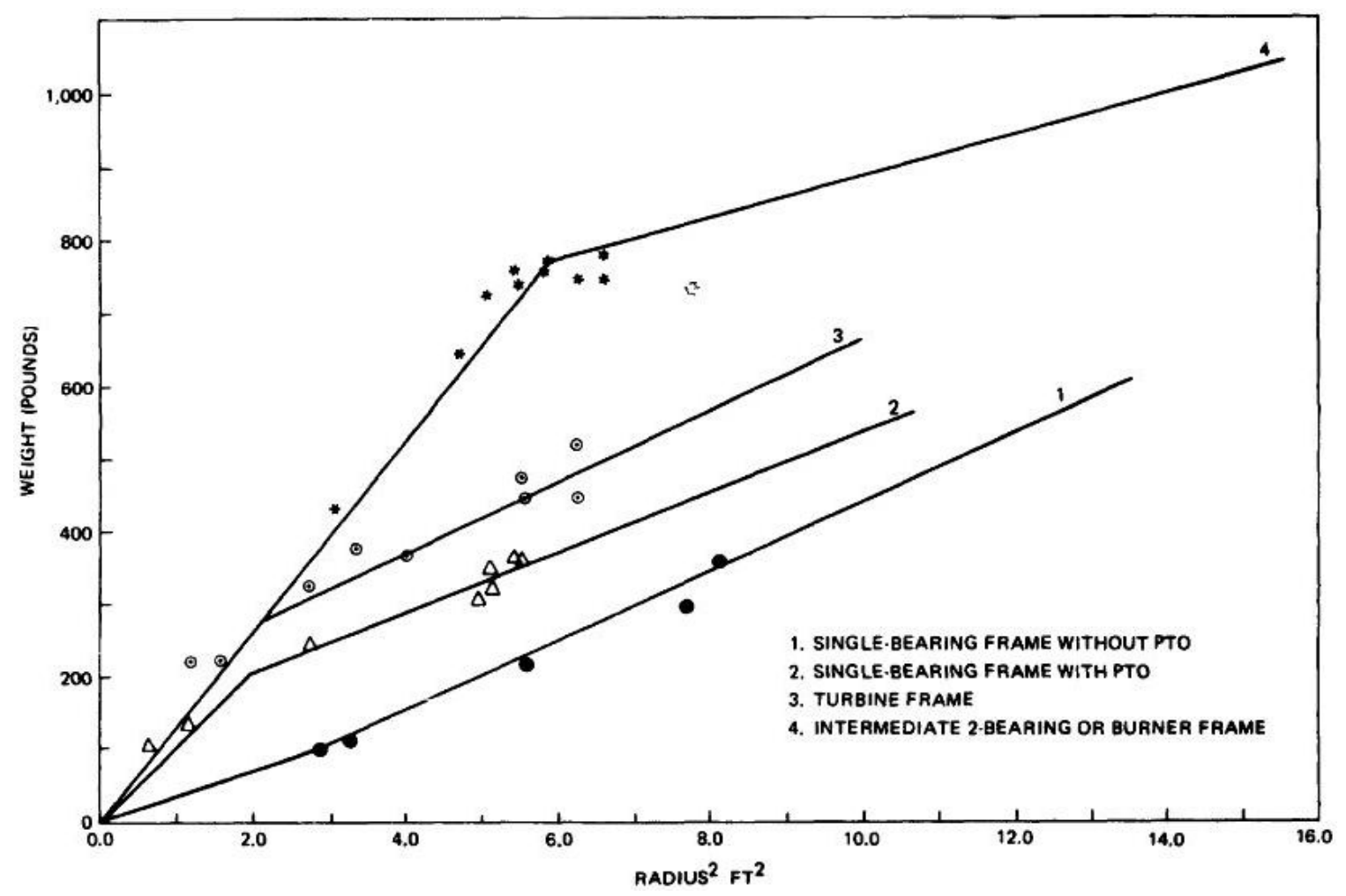

Figure 24. Frame Weight versus Engine Radius ${ }^{\text {xvi }}$.

These curves were transformed into curve-fit equations for use in the HAPSS code. The user can specify any number of frames and the type of frame for each. For the propulsive fans in hybrid system however, the user will likely only choose one or two Type 1 frames. Nevertheless, the additional frame options will allow for greater flexibility of the code in the future.

\subsection{D Fan Analysis}

The 2D fan performance analysis considers the performance of the fan in two dimensions by analyzing the flow characteristics through the rotor and stator. The 2D fan performance analysis utilizes outputs from the 1D analysis such as fan diameter and speed. Using these parameters and other user specified variables, the 2D fan analysis looks at both the performance of the fan in two dimensions and determines basic blade geometry and dimensions. 


\subsubsection{Coordinate System}

The $2 \mathrm{D}$ analysis of the fan begins by establishing a coordinate system. The chosen system and nomenclature appear to be well accepted throughout the industry, and is shown here in Figure 25.

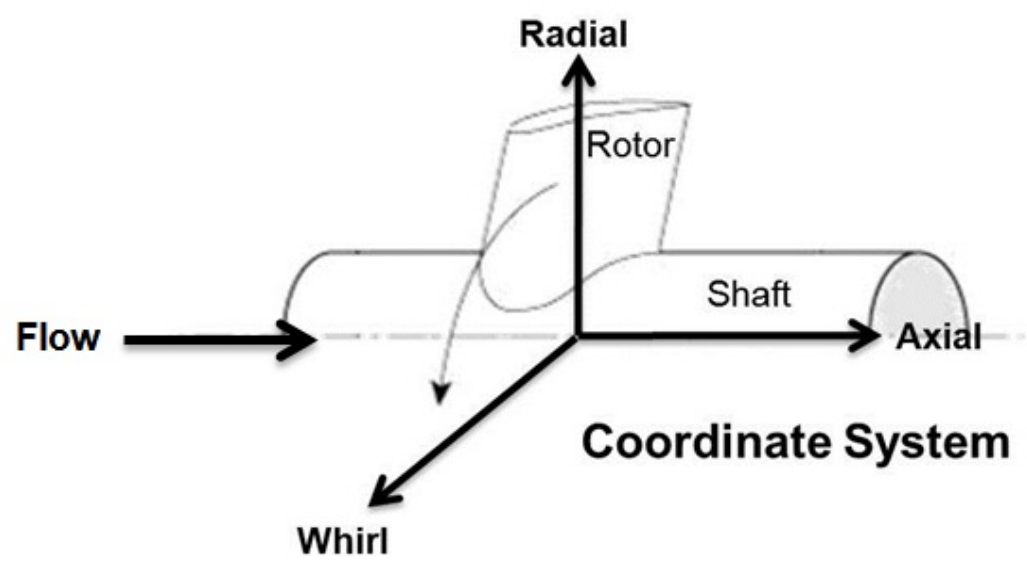

Figure 25. Rotor Coordinate System.

In this figure, the air flows through the rotor from left to right in the positive axial direction along the axis of rotation. The positive radial direction is normal to the axis of rotation and passes through the blade. In other words, the radial direction is orthogonal to the blade cross section. Finally, the positive whirl direction is also normal to the axis of rotation, but points in the direction of blade motion. The 2D analysis focuses on the flow in the axial-whirl plane.

Even through the overall flow through the rotor is in the axial direction, the air velocity will have components in the whirl and radial directions which must be studied. Considerations in the radial direction would occur in a 3D analysis where flow variations from hub to tip are estimated. In this version of HAPSS 3D flow effects are not considered. The 2D analysis is performed assuming an infinite cascade of blades of infinite length and constant cross section. For this reason, the $2 \mathrm{D}$ analysis is said to occur at the mean fan diameter, or halfway between the hub and the tip. With a hub-to-tip ratio of 0.3 for example, the mean diameter exists at $65 \%$ of the overall fan diameter. 


\subsubsection{Velocity Triangles}

The driving force behind the $2 \mathrm{D}$ analysis is the velocity triangle diagram. Using the blade speed, axial velocity into the rotor, and work done by the rotor, the velocity triangles can be constructed and then used to make performance predictions. Figure 26 shows typical velocity triangles labeled using common cascade notation from Gas Turbine Theory by Saravanamuttoo, Rogers, and Cohen, $2001^{\text {xviii }}$. In this figure, flow travels from left to right.

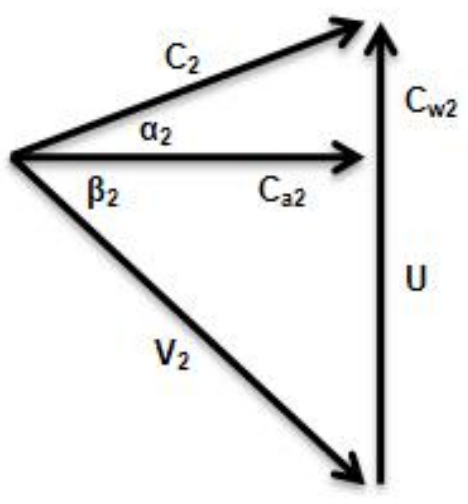

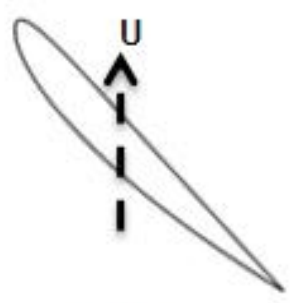

Rotor
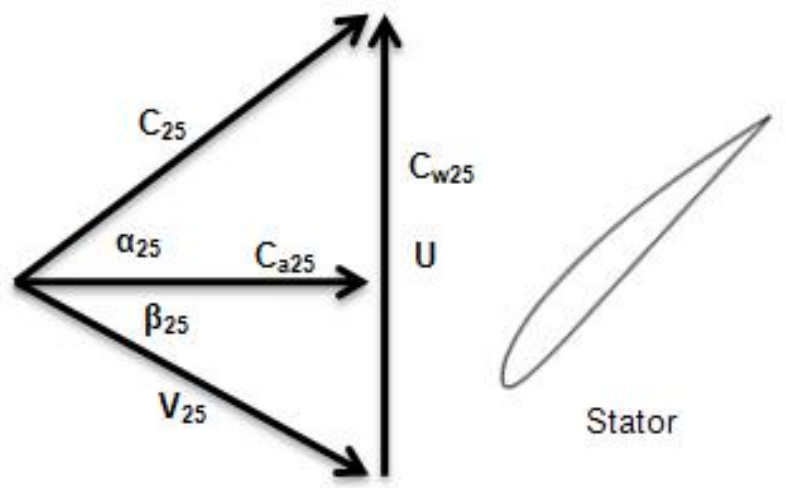

Figure 26. Velocity Triangles using Cascade Notation.

The subscripts " 2 " and " 25 " denote the rotor entrance and exit, respectively, as in the 1D analysis. The definitions of each vector and angle are as follows.

- $\quad V=$ Relative velocity

- $\quad C=$ Absolute velocity

- $\quad C_{a}=$ Axial velocity

- $\quad C_{w}=$ Whirl velocity

- $\quad U=$ Blade velocity

- $\quad \alpha=$ Absolute air flow angle

- $\beta=$ Relative air flow angle

The absolute velocity represents the air as it actually flows through the rotor, while the axial and whirl velocities represent the perpendicular components of the absolute velocity. Finally, 
the relative velocity represents the flow as seen by the moving rotor. Because it best represents the flow through the rotor, the relative velocity is of most use in the $2 \mathrm{D}$ performance analysis.

To reasonably perform the $2 \mathrm{D}$ fan performance analysis, some simple assumptions must be made. First, the axial velocity is said to remain constant throughout the stage including the rotor and stator $\left(C_{a 2}=C_{a 25}=C_{a 3}\right)$. Second, the air is said to exit the stator with the same geometry as which it entered the rotor $\left(\alpha_{3}=\alpha_{2}, C_{3}=C_{2}\right)$. With these assumptions the analysis of the rotor performance using the velocity triangles is greatly simplified.

Parameters required to perform the analysis include rotor inlet absolute velocity, inlet absolute flow angle, and the blade speed. Both rotor inlet absolute velocity and the blade speed are outputted by the 1D fan analysis. The inlet absolute flow angle can be assumed to be zero if not inlet guide vanes are present.

The $2 \mathrm{D}$ analysis of the rotor began at the rotor inlet. First, the whirl and axial components of the flow entering the inlet are determined using the following equations.

$$
\begin{aligned}
& C_{w 2}=C_{2} \sin \left(\alpha_{2}\right) \\
& C_{a 2}=C_{2} \cos \left(\alpha_{2}\right)
\end{aligned}
$$

These equations imply that if the air enters the rotor in the pure axial direction $\left(\alpha_{2}=0^{\circ}\right)$ then there is no whirl velocity and that the axial velocity is equal to the absolute velocity. It should be noted now that the $2 \mathrm{D}$ performance analysis was executed assuming pure axial inlet flow. Using the blade velocity from the 1D analysis, the rotor inlet relative air flow angle, relative flow velocity, and relative Mach number are calculated using the following equations.

$$
\begin{gathered}
\beta_{2}=\operatorname{atan}\left(\frac{U}{C_{a 2}}-\tan \left(\alpha_{2}\right)\right) \\
V_{2}=\frac{C_{a 2}}{\cos \left(\beta_{2}\right)} \\
M_{r e l}=\frac{V_{2}}{a_{2}}
\end{gathered}
$$

where $a_{2}$ is the acoustic speed before the rotor, calculated during the $1 \mathrm{D}$ fan analysis. Figure 27 shows an example of the relation between the mean diameter inlet relative Mach number, tip Mach number, and the inlet airflow angle. The red circle indicates a likely design point where the tip Mach number is 1.0 and the inlet airflow angle is zero (no inlet guide vanes). These contours 
will vary based on the fan design and the operating point, but the overall behavior will remain the same.

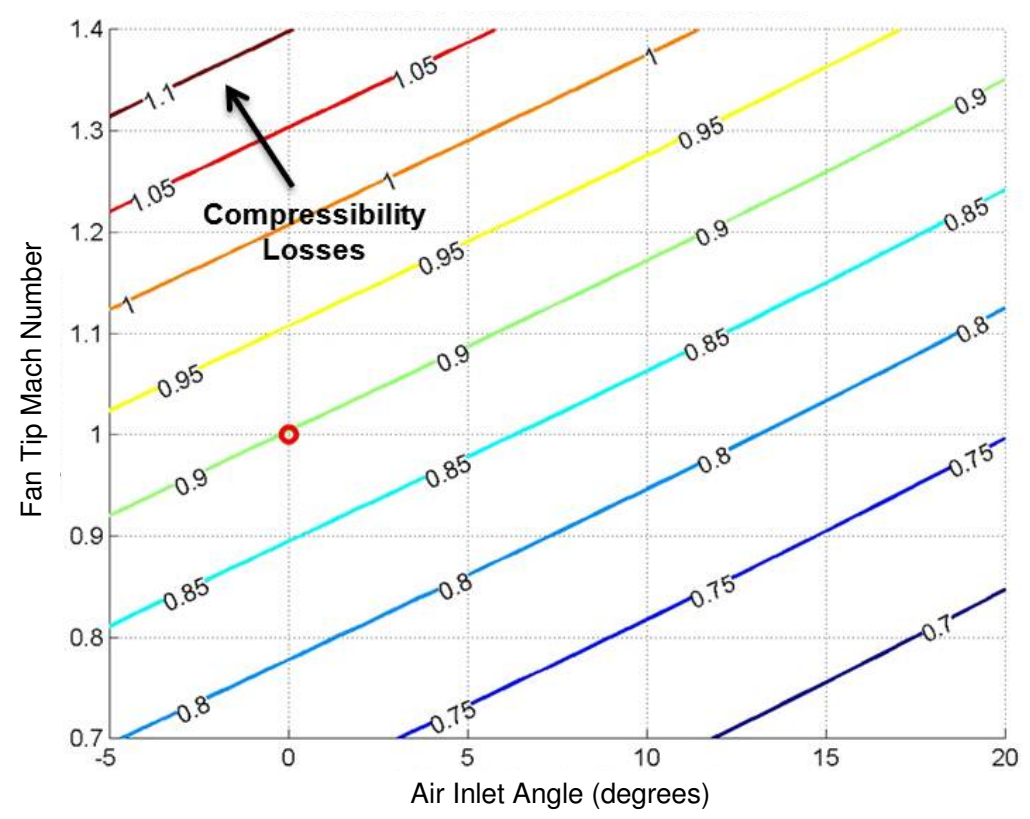

Figure 27. Relative Inlet Mach Number vs. Tip Mach Number and Air Inlet Angle.

The relative Mach number is an extremely important value to consider as it is a good predictor of the onset of compressibility effects $\left(M_{\text {rel }}>1\right)$. Saravanamuttoo suggests that modern high bypass ratio turbofans can achieve relative Mach numbers of 1.4-1.5 without experiencing significant compressibility losses. Fans and compressors with high relative Mach numbers should have special considerations for blades that are designed to cope with transonic and supersonic flows.

With the rotor inlet velocity triangle calculated, the rotor exit velocity triangle can now be derived. Because the blade velocity and axial velocity remain constant in the rotor, the unknowns that must be solved for are the rotor exit absolute and relative angles. Using these angles, the whirl, absolute, and relative velocities can be found easily.

In order to determine the rotor exit flow angles, the power inputted to the rotor, as calculated in the 1D fan analysis, is considered. The energy inputted to the rotor is transferred to the fluid by changing its angular momentum. Thus, the flow angles of the air can be calculated using the rotor power. Equation (4.7.2.6) shows the calculation of power using the mass flow rate 
and change in total enthalpy. Here, the enthalpy rise is put into terms of the change in whirl velocity and then the exit angles.

$$
\begin{gathered}
P w r=\dot{m}_{f} \Delta H \\
=\dot{m}_{f} U\left(C_{w 25}-C_{w 2}\right) \\
=\dot{m}_{f} U C_{a}\left(\tan \left(\alpha_{25}\right)-\tan \left(\alpha_{2}\right)\right) \\
=\dot{m}_{f} U C_{a}\left(\tan \left(\beta_{2}\right)-\tan \left(\beta_{25}\right)\right)
\end{gathered}
$$

With rotor power defined as a function of the relative and absolute exit flow angles, these angles can be calculated. The rotor power is calculated during the 1D fan analysis in BTU/s.

$$
\begin{aligned}
& \beta_{25}=-\operatorname{atan}\left(\frac{P w r}{\dot{m}_{f} U C_{a}}-\tan \left(\beta_{2}\right)\right) \\
& \alpha_{25}=\operatorname{atan}\left(\frac{P w r}{\dot{m}_{f} U C_{a}}+\tan \left(\alpha_{2}\right)\right)
\end{aligned}
$$

All of the velocities and angles are now known to build the full velocity triangles. These triangles offer a designer valuable knowledge of the 2D flow characteristics through the fan stage. Some of the velocity triangle information can also be used to calculate other performance specifications for the fan.

\subsubsection{Stage Performance}

With the velocity triangles determined, various performance parameters can then be calculated, the first of which is the stage temperature rise. The energy inputted by the fan is absorbed usefully in raising the pressure of the flow between the rotor inlet and exit. However, some of the inputted energy is wasted to overcome friction losses. Nevertheless, the whole of the input power reveals itself in the stage temperature rise, shown in the following equation.

$$
\Delta T_{f}=T_{25}-T_{2}=\frac{U c_{a}}{c_{p}}\left(\tan \left(\beta_{2}\right)-\tan \left(\beta_{25}\right)\right)
$$

Using the stage temperature rise, the stage pressure ratio can be calculated using the following equation.

$$
\Delta P_{f}=\left[1+\frac{\eta_{s} \Delta T}{T_{2}}\right]^{\frac{\gamma}{\gamma-1}}
$$


where $\eta_{s}$ is the isentropic efficiency of the compression. It can be seen that the fan pressure ratio is highly dependent on the efficiency. In order to achieve a high temperature rise, the fan must exhibit a suitable combination the following three properties.

- High blade speed

- High axial velocity

- High fluid deflection in the rotor

\subsubsection{Blade Speed and Stress}

The blade speed will be limited by the tensile stress in the material due to centrifugal forces. Excessive blade speed by definition can also lead to high relative Mach numbers at the blade tip causing excessive compressibility losses. The blade tensile stress is calculated using the following equation

$$
\sigma_{\max }=\frac{\rho_{b} \omega}{a_{r}} \int_{r}^{t} A r d r
$$

where $\rho_{b}$ is the blade material density, $\omega$ is the angular velocity of the fan, $A$ is the blade cross sectional area, $r$ is the radius, and subscripts $r$ and $t$ denote the blade root and tip. This equation can be simplified into two other forms assuming a constant blade cross section.

$$
\begin{gathered}
\sigma_{\text {max }}=2 \pi N^{2} \rho_{b} A \\
\sigma_{\text {max }}=\frac{\rho_{b}}{2} U_{t}^{2}\left[1-h t r^{2}\right]
\end{gathered}
$$

where $N$ is the fan rotational speed, $A$ is the annulus area, $U_{t}$ is the blade tip speed, and $h t r$ is the hub-to-tip ratio. Finally, if the cross sectional area is varied linearly from root to tip, the tensile stress equation takes on the following form.

$$
\begin{aligned}
& \sigma_{\max }=\frac{\rho_{b}}{2} U_{t}^{2}\left[1-h t r^{2}\right] K \\
& K=1-\left[\frac{(1-d)\left(2-h t r-h t r^{2}\right)}{3\left(1-h t r^{2}\right)}\right]
\end{aligned}
$$

where $d$ is the ratio of the blade cross sectional area at the tip and root. Saravanamuttoo notes that typical values for $K$ fall between 0.55 and 0.65 for tapered blades ${ }^{x v i i i}$. At low hub-tip ratios the blade stress is of large concern. However, without knowledge of the blade cross sectional area or the material chosen for the blades, it is difficult to predict the blade stresses at this time. 
Moreover, with lighter blade materials and technologies, such as carbon fiber, compressibility effects will usually limit the fan's design before blade stress.

\subsubsection{Inlet Axial Velocity}

The second means to increase the stage temperature rise is to increase the axial velocity. The higher the velocity, the greater the air flow rate through the fan. An increase in the fan face Mach number results in a decrease in the fan diameter due to the higher mass flow rate per unit area. Today's turbofan engines typically have a fan face Mach number in the 0.5-0.6 range. Increasing the axial velocity directly elevates the relative Mach number and potentially leads to compressibility losses. Figure 28 illustrates the relation between relative Mach number, axial velocity, and blade speed for a compressor without inlet guide vanes.

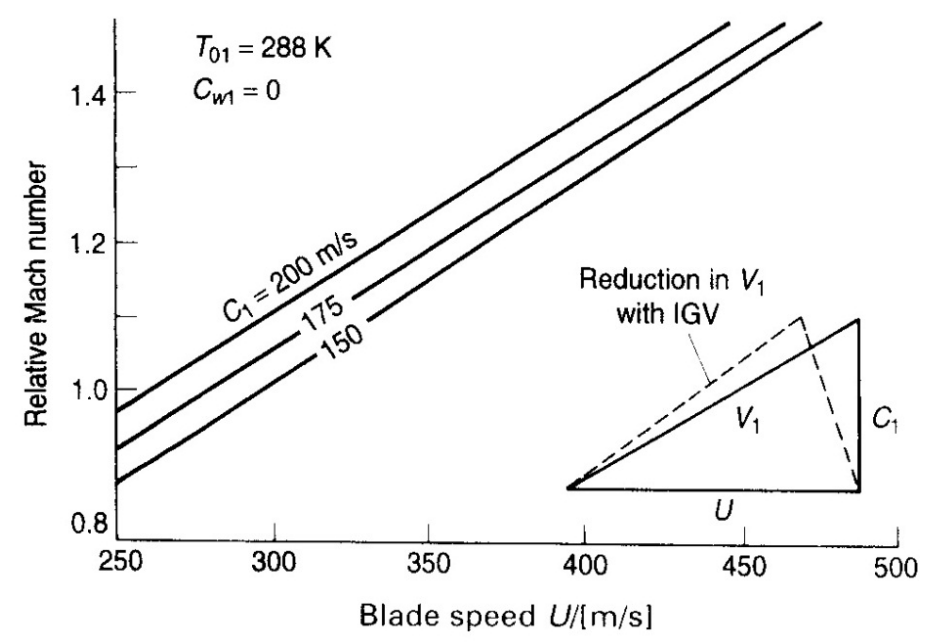

Figure 28. Relative Mach Number vs. Blade Speed and Axial Velocity at Rotor Inlet ${ }^{\text {xviii }}$.

The use of inlet guide vanes allows for the reduction in relative Mach number by imparting an angle on the flow before it enters the rotor. Inlet guide vanes were common prior to the 1950's to cope with transonic velocities in the fan. However, modern transonic blading has allowed relative Mach numbers to reach 1.5 in some high bypass ratio turbofans. Inlet guide vanes also add weight and complexity to the propulsion system. For these reasons, guide vanes are rarely used in modern turbofan engines. 


\subsubsection{Fluid Deflection and Diffusion}

The third means to increase stage temperature ratio is to increase the fluid deflection in the rotor. Recall the velocity triangles shown in Figure 26. Given a fixed inlet relative flow angle $\beta_{2}$, the fluid deflection is increased by reducing the outlet relative flow angle $\beta_{25}$, which in turn increases the change in whirl velocity $\Delta C_{w}$ and reduces the outlet relative flow velocity $V_{25}$. This reduction in the outlet relative flow velocity is referred to as diffusion and can lead to excessive losses in the rotor. An early and simple predictor of diffusion is the de Haller number, shown here in the following equation.

$$
D H N=\frac{V_{25}}{V_{2}}>0.72
$$

This equation states that the ratio of the outlet to inlet relative flow velocities must be greater than 0.72 to avoid high diffusion rates. Both the fan tip Mach number and the rotor airflow inlet angle will have a significant impact on the de Haller number. An example of this effect is shown in Figure 29, and this particular example, the de Haller number does not fall below 0.72 . Again, these contours will vary based on the fan design and the operating point, but the overall behavior will remain the same.

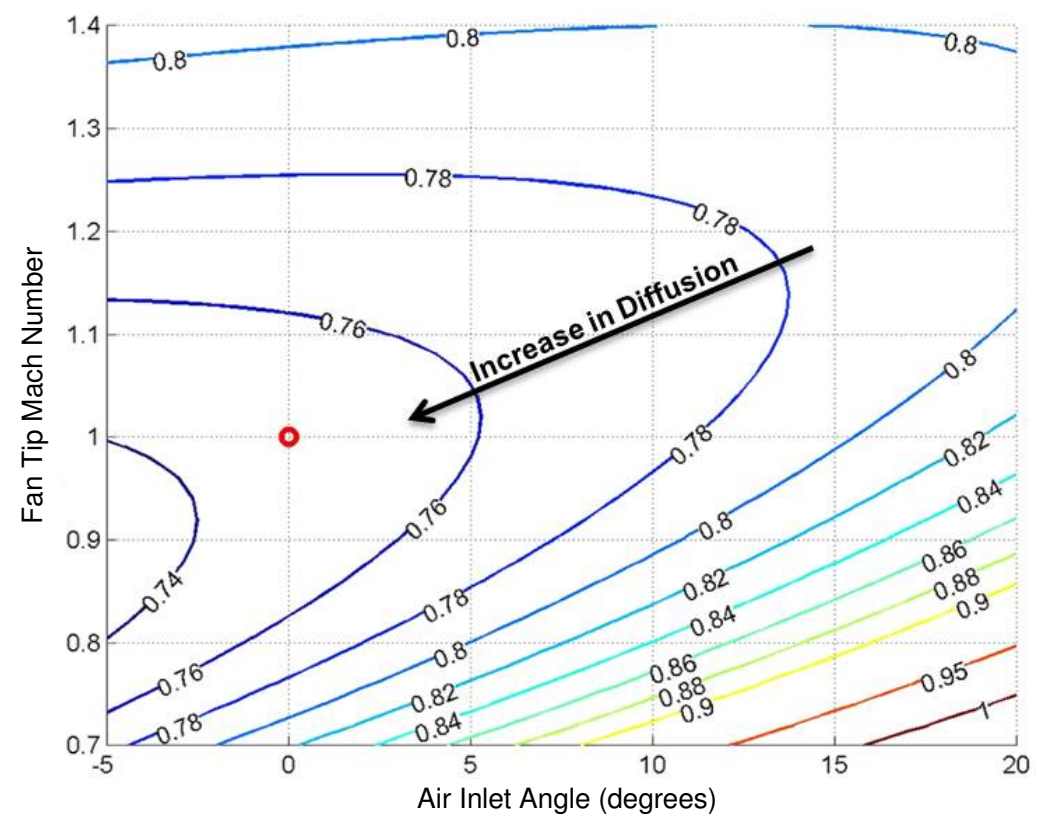

Figure 29. de Haller Number vs. Tip Mach Number and Air Inlet Angle.

As expected, both tip Mach number and air inlet angle have a significant effect on the rate of diffusion in the rotor. Higher tip Mach numbers and higher inlet air angles both appear to 
reduce diffusion. As mentioned before however, higher tip Mach numbers can cause excessive compressibility losses while any non-zero air inlet angle requires the use of inlet guide vanes.

Another estimator of diffusion is the NACA diffusion factor which is widely used in the industry. The formulation of the diffusion factor is based on the velocity distribution over the suction and pressure sides of the blades. An illustration of this velocity distribution is shown in Figure 30.

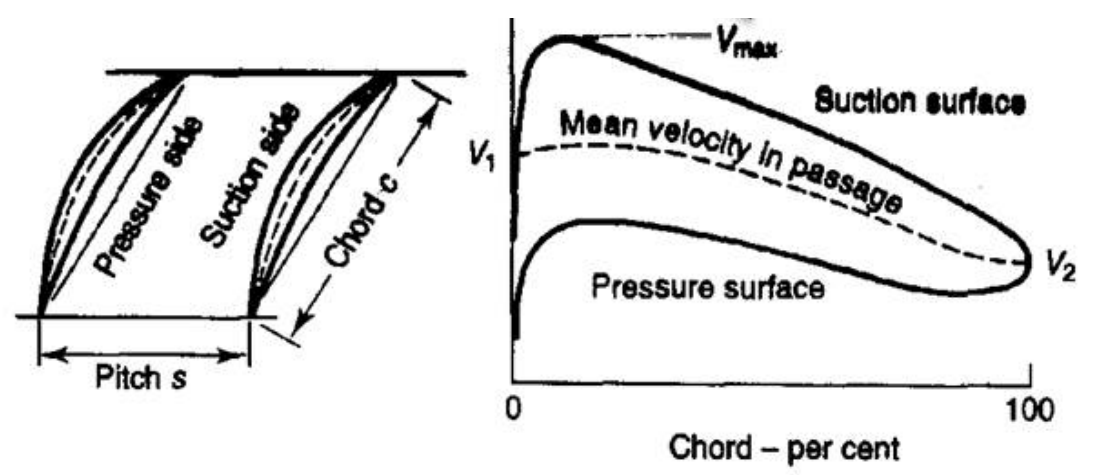

Figure 30. Rotor Blade Velocity Distribution ${ }^{\text {xvIII. }}$

The losses in the rotor increase as the boundary layers over the suction and pressure sides of the blades grow in thickness. Thicker boundary layers occur in regions of high velocity gradients, the largest of which tends to occur at $10-15 \%$ of the chord on the suction side of the blade as shown in Figure 30. Thicker boundary layers can lead to blockage between the blades, local drops in stagnation pressure, and even flow separation and stall in extreme scenarios. Through cascade testing, it was determined that the maximum velocity on the suction surface of the blade can be predicted by the following equation.

$$
\mathrm{V}_{\text {max }} \approx V_{25}+\left(\frac{\Delta C_{w_{c}}^{\frac{S}{c}}}{2}\right)
$$

where $s$ is the blade pitch and $c$ is the blade chord. $s / c$ is commonly referred to as the pitch/chord ratio and is the inverse of solidity, another common variable for fan and compressor design. Figure 31 , based on fan test results, compares the pitch/chord ratio to the nominal air deflection $\left(\varepsilon^{*}\right)$ and air outlet angle. This figure can allow the user to pick an initial solidity for the fan. 


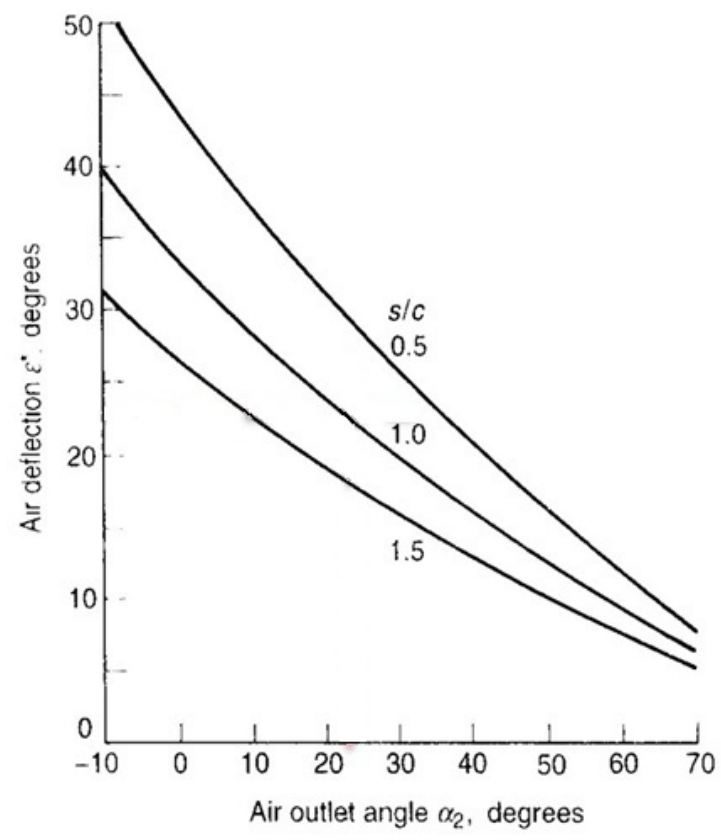

Figure 31. Rotor/Stator Solidity for Varying Air Deflection and Outlet Angle ${ }^{\text {xviii }}$.

In the design process, the nominal air deflection is assumed to be $80 \%$ of the stalling deflection. The stalling deflection is said to occur when the loss reaches twice its minimum value. For simplicity purposes, it can be assumed that the nominal air deflection equals the air deflection calculated from the velocity triangles. The air deflection is simply the difference between the absolute air inlet and exit angles, as shown in the following equation.

$$
\varepsilon=\alpha_{2}-\alpha_{25}
$$

The NACA diffusion factor is calculated by the following equation, into which the maximum velocity can be substituted along with the chosen pitch/chord ratio and necessary velocities from the velocity triangles.

$$
D F \approx \frac{V_{\max }-V_{25}}{V_{2}} \approx 1-\frac{V_{25}}{V_{2}}+\frac{\Delta C_{w}}{2 V_{2}} \frac{S}{c}
$$

Acceptable values for the diffusion factor were determined through cascade testing and are reliable for subsonic and slightly supersonic relative Mach numbers. Figure 32 shows a compilation of cascade testing data comparing the total pressure loss coefficient to the NACA diffusion factor. Figure 33, shows an identical plot summarized into two distinct sections. 


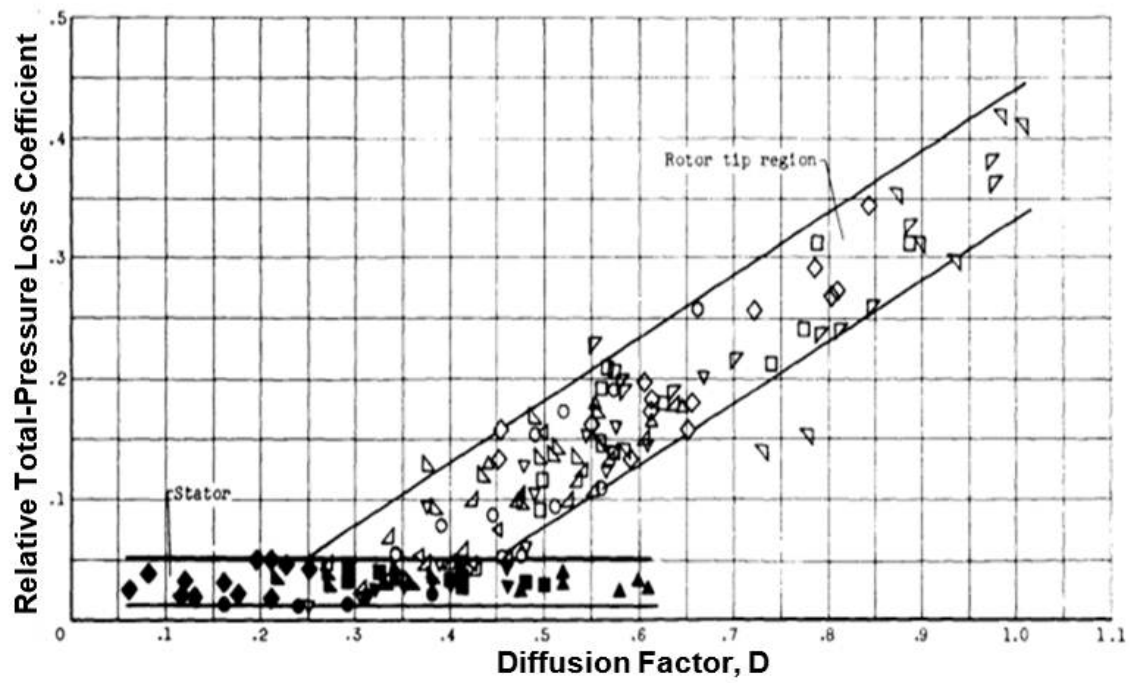

Figure 32. Total Pressure Loss Coefficient vs. NACA Diffusion Factor ${ }^{\mathrm{xix}}$.

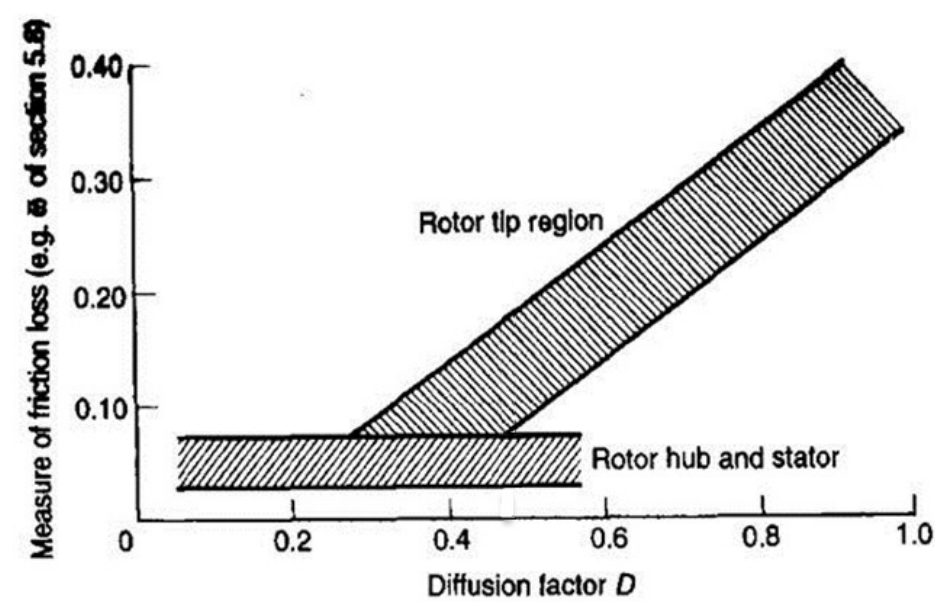

Figure 33. Measure of Suction Loss vs. NACA Diffusion Factor ${ }^{\text {xviii. }}$

In the stator and the hub region of the rotor, losses are minimal up to a diffusion factor of about 0.6 . In the tip region of the rotor however, the losses increase rapidly after a diffusion factor of about 0.4 . Because the $2 \mathrm{D}$ analysis is performed at the mean fan diameter, a diffusion factor under 0.5 would be acceptable. Future iterations of HAPSS will provide $2 \mathrm{D}$ analyses at both the hub and tip regions of the rotor as well.

Similar to the de Haller number, it was desired to know what affect the tip Mach number and inlet air angle has on the NACA diffusion factor. This trade is shown here in Figure 34. 


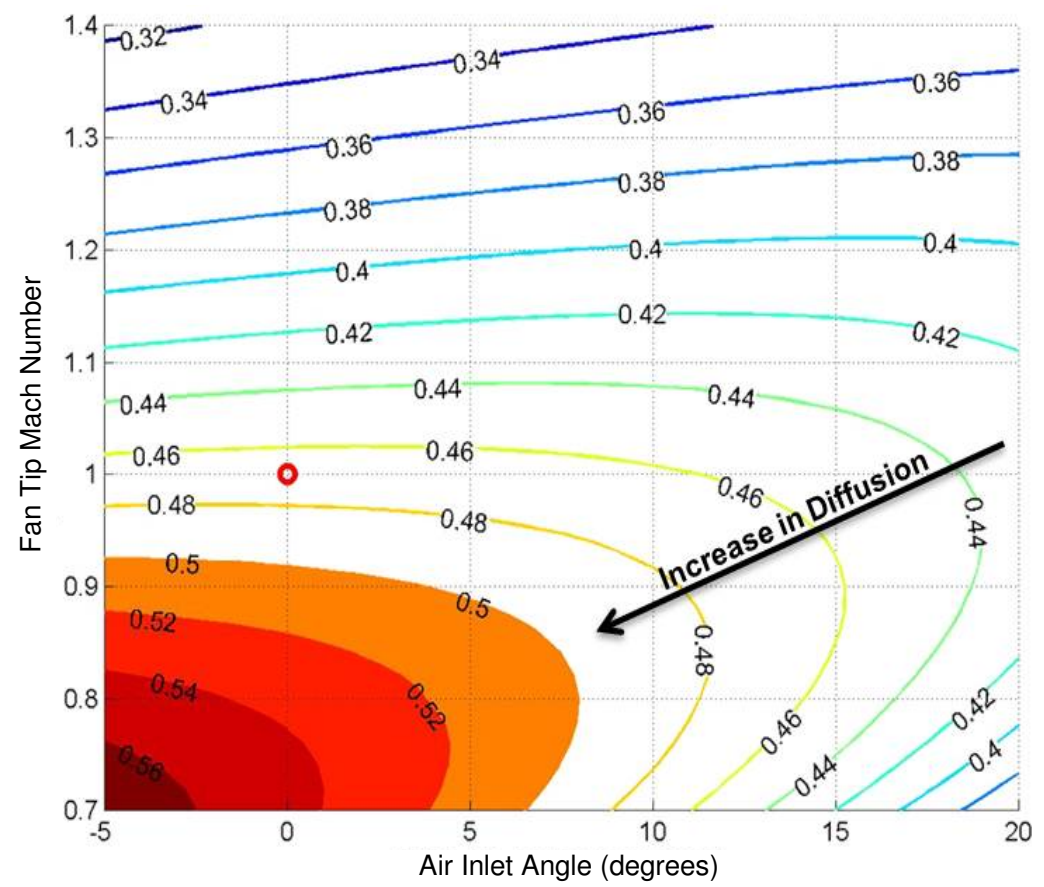

Figure 34. NACA Diffusion Factor vs. Tip Mach Number and Air Inlet Angle.

This figure shows a remarkably identical trend as the de Haller number in the same design space. This is a very reassuring conclusion as both parameters are designed to estimate diffusion losses in the rotor and stator. However, in this example the de Haller number did not reveal any combinations of tip Mach number and inlet air angle that would result in excessive diffusion. The diffusion factor however does show a region, indicated in orange to dark red, where diffusion could lead to heavy losses in the rotor. Similar to the de Haller number, both tip Mach number and air inlet angle have a significant effect on the diffusion factor, and once again, increases in either help to reduce diffusion.

\subsubsection{Degree of Reaction}

Another performance measurement of the rotor is the degree of reaction. This parameter is a measure of how much the rotor contributes to the overall static pressure rise in the stage. The degree of reaction is most accurately calculated using the static enthalpy rise of the rotor and stator as shown in the following equation.

$$
\Lambda=\frac{\Delta h_{\text {rotor }}}{\Delta h_{\text {stator }}}
$$


However, with a negligible change in the specific heat at constant pressure through the stage, the degree of reaction can be put in terms of temperature. Finally, using the relationship between rotor input power and the velocity triangles, the degree of reaction can be put into terms of the outlet relative flow angles as shown here

$$
\Lambda=\frac{C_{a}}{2 U}\left(\tan \left(\beta_{2}\right)+\tan \left(\beta_{25}\right)\right)
$$

The degree of reaction varies between 0 and 1, where 0 represents a pure impulse rotor, and 1 represents a pure impulse stator. An impulse style rotor for example has the same inlet and exit passage area and the static pressure rise occurs solely in the stator. Figure 35 shows an example of the variation in the degree of reaction based on tip Mach number and inlet air angle.

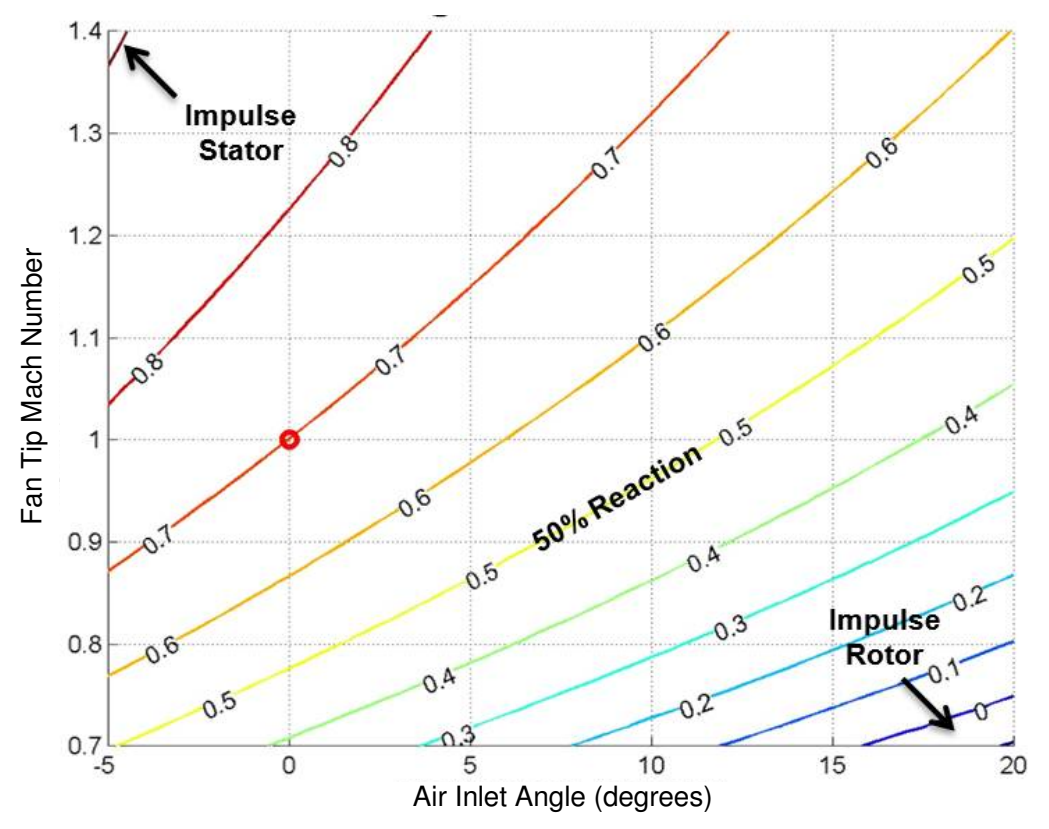

Figure 35. Degree of Reaction vs. Tip Mach Number and Air Inlet Angle.

A degree of reaction of 0.5 is referred to as $50 \%$ reaction and in many cases is desirable as it allows the diffusion to be shared between the rotor and the stator. Figure 36 shows the green dashed $50 \%$ reaction line drawn into the plot of NACA diffusion factor. 


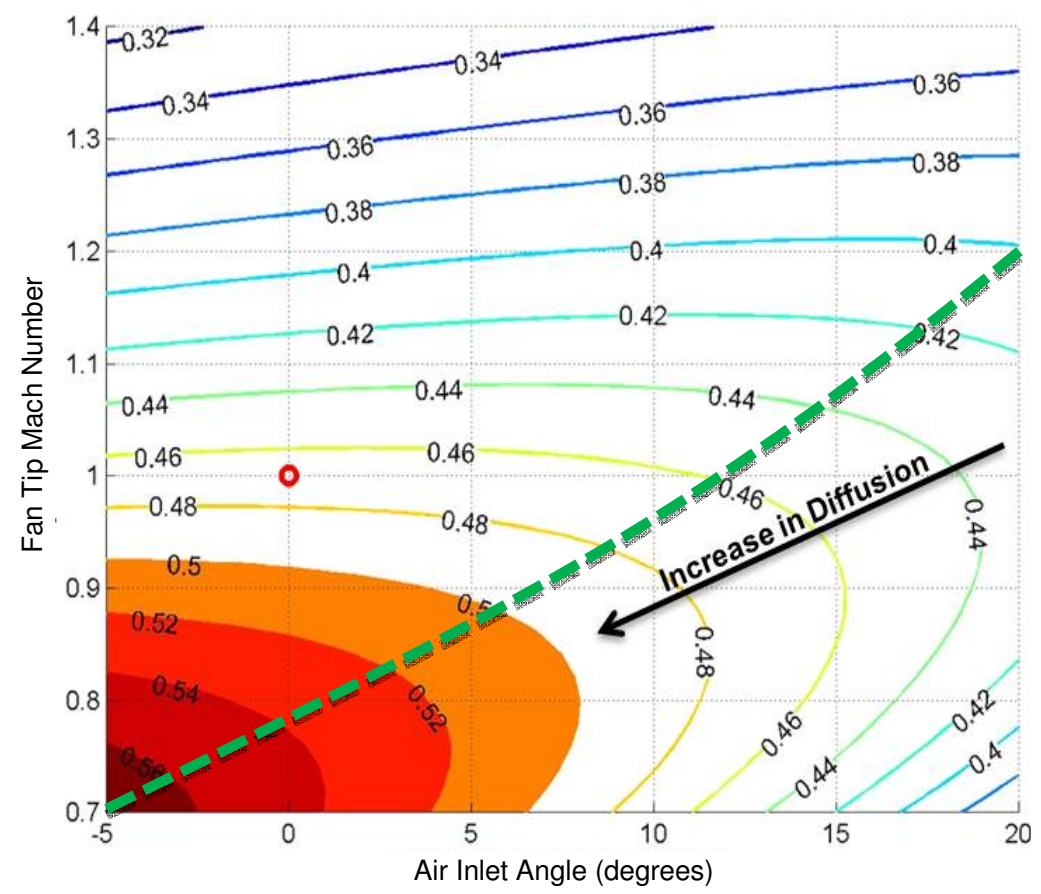

Figure 36. NACA Diffusion Factor with $50 \%$ Reaction Line.

This figure reveals that the $50 \%$ reaction is near the peaks of minimum diffusion, and that $40 \%$ reaction in this example would yield the lowest diffusion. Achieving $50 \%$ reaction however is rarely a leading driver in the design of a fan since the performance and efficiency of the fan are more highly dependent on other parameters. The degree of reaction also tends to vary greatly in the radial direction across the annulus. Table 5 shows the final performance results from the $2 \mathrm{D}$ analysis at the on-design point of top-of-climb.

\subsubsection{D Fan Blade Design}

Once the 2D analysis is completed, HAPSS performs an elementary 2D blade design analysis. When beginning this process it became apparent that the design of the blade cross section is rooted deeply in empirical data and experimental results. It was also discovered that reliable preliminary blade designs can indeed be created using empirical results. On the other hand, contemporary blade designs with compound curves and exotic cross sections undoubtedly require heavy use of CFD, testing, and optimization techniques. Figure 37 compares a fan blade from the General Electric GEnx turbofan to the one designed in this phase of work. 

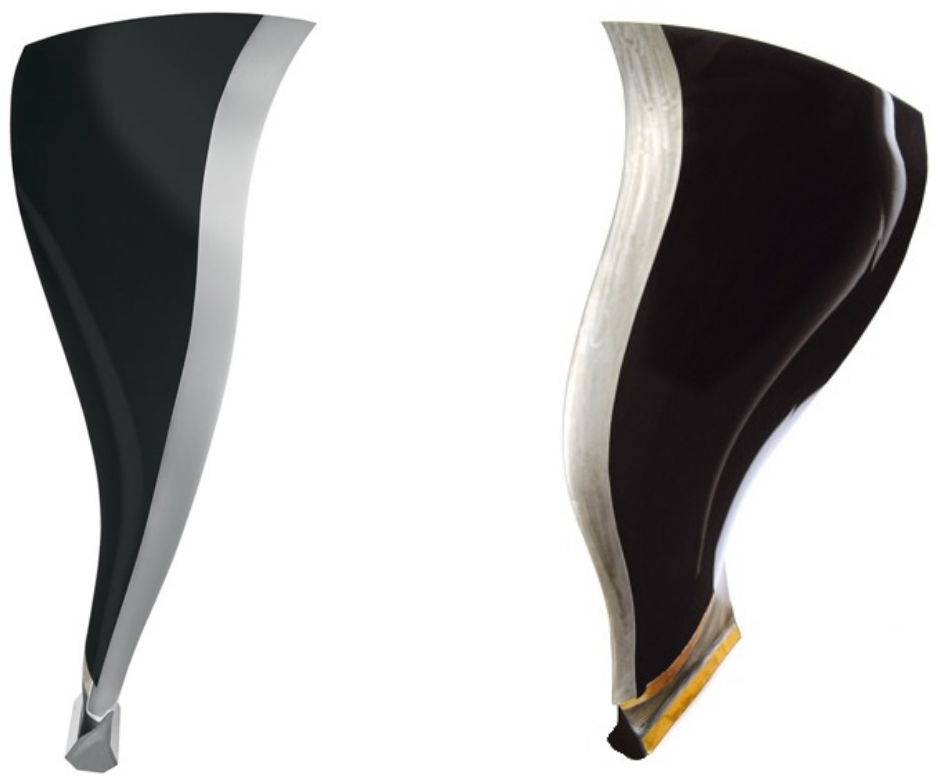

Figure 37. General Electric GEnx $\left(\right.$ left $\left.^{\mathrm{xx}}\right)$ and GE90 $\left(\right.$ right $\left.^{\mathrm{xi}}\right)$ Fan Blades.

It is readily obvious that the both the GEnx and GE90 fan blades required an extensive use of CFD and testing to reach its final design. However, it is likely that these blade design started life as a simple 2D analysis using empirical data. Given the level of depth of the HAPSS program, neither CFD nor optimization is used in this elementary fan blade design.

\subsubsection{Blade Cross Section Notation}

To begin the fan blade design process, the important parameters were determined and drawn onto a generic fan blade shown here in Figure 38. The variables used are analogous to the cascade notation utilized in the 2D performance analysis. 


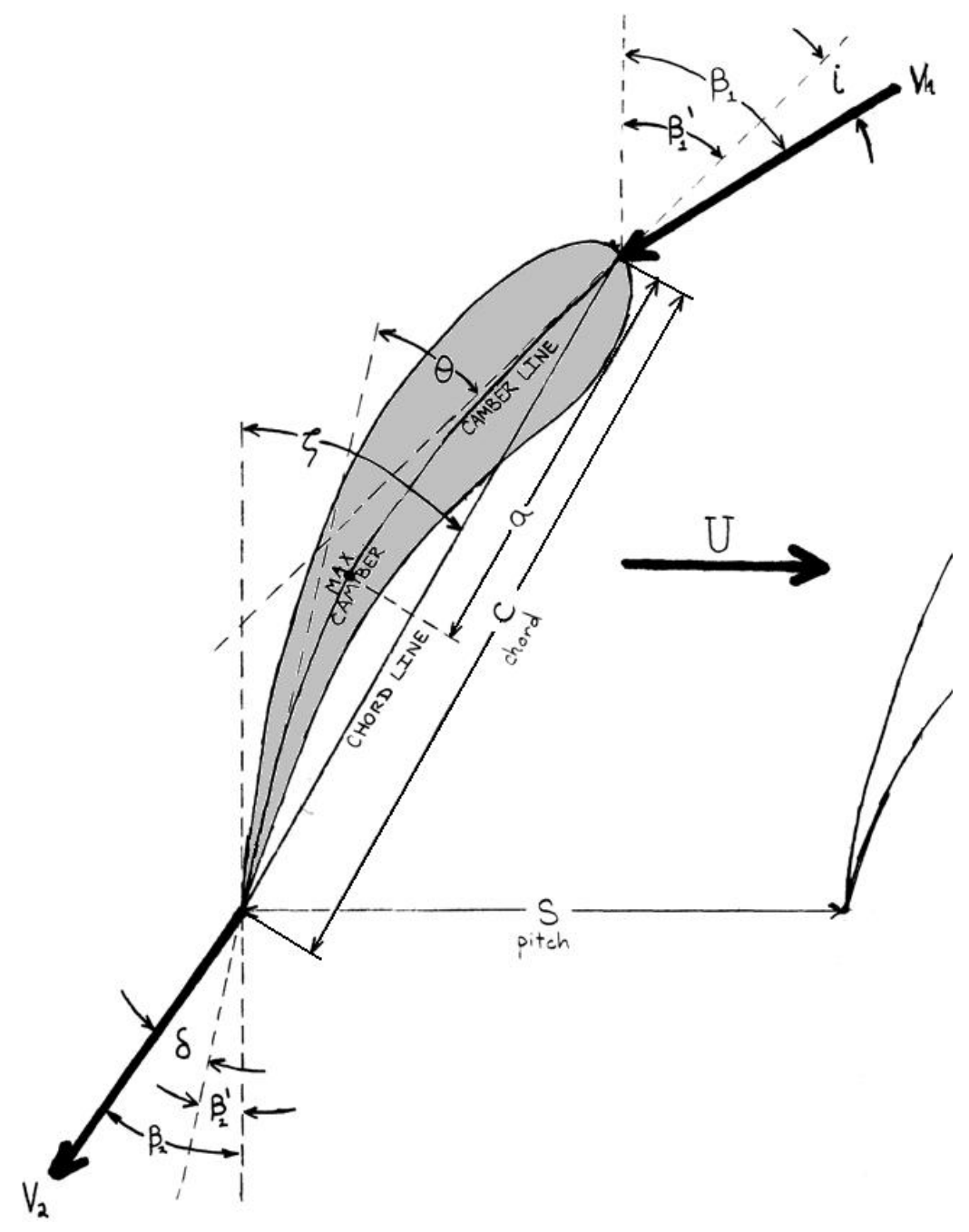

Figure 38. Fan Blade Design Using Cascade Notation.

This figure shows a top view of a generic blade cross section. In this view, the flow would enter from the top of the page and the blade would move from left to right in the $U$ vector direction. The variables in this figure are defined as follows:

- $\quad V_{1}=$ Inlet relative flow velocity

- $\quad \beta_{1}=$ Inlet relative flow angle

- $\quad \beta^{\prime}{ }_{1}=$ Inlet camber angle

- $\quad i=$ Incidence (angle off camber)

- $\theta=$ Blade camber angle

- $\xi=$ Blade stagger angle

- $\quad V_{2}=$ Outlet relative flow velocity
- $\beta_{2}=$ Outlet relative flow angle

- $\quad \beta_{2}^{\prime}=$ Outlet camber angle

- $\delta=$ Deviation (angle off camber)

- $\quad c=$ Blade chord

- $\quad s=$ Blade pitch

- $a=$ Chord location of maximum camber

- $\quad U=$ Blade velocity 
It should be noted that this figure uses subscripts "1" and " 2 " to mean rotor inlet and outlet, respectively. In the prior and future analysis however " 2 " and " 25 " are used to represent the rotor inlet and exit.

\subsubsection{Blade Shape}

Due to the high relative flow velocities that the fan will encounter, a unique blade shape was chosen upfront unlike the cambered shape pictured in Figure 38. The chosen blade shape is commonly referred to as a double-circular-arc section and is ideal for transonic and supersonic fan and compressor blading. This blade shape is shown here in Figure 39.

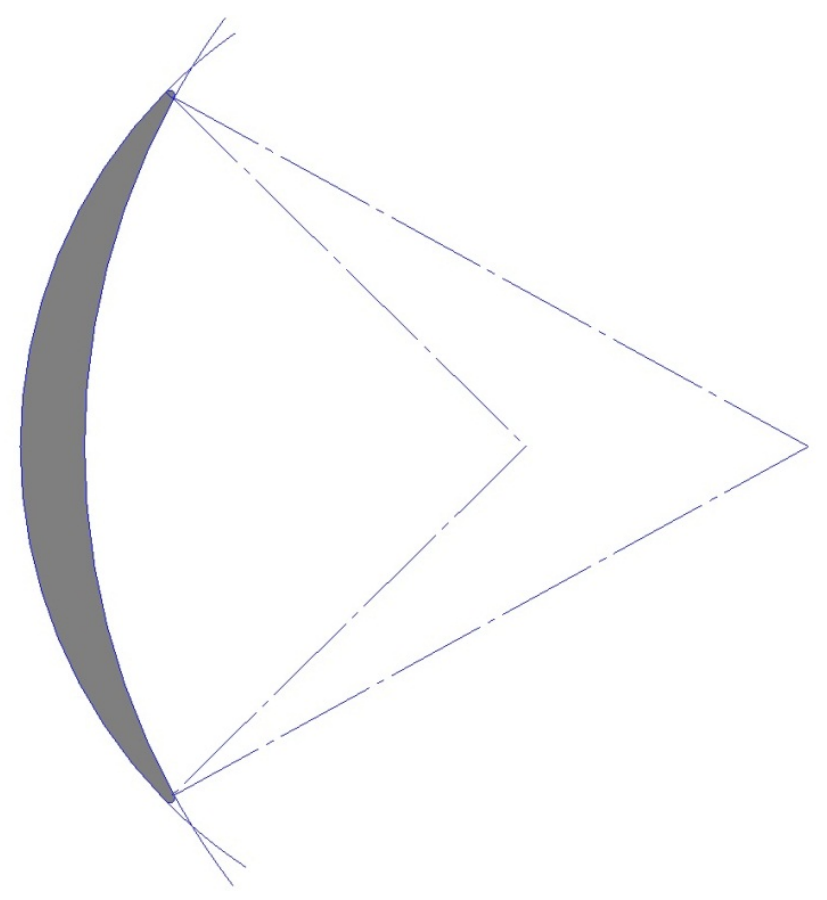

Figure 39. Example Double-Circular-Arc Blade Cross Section.

As the name suggests, this type of blade is created by two intersecting circular arcs of different radii. The radius and center of the arcs will vary depending on the desired camber angle, chord, etc. The main difference between double-circular-arc blades and standard cambered airfoil blades is that the location of max camber is typically at $50 \%$ chord, where cambered airfoil blades have their max camber much closer to the leading edge. 


\subsubsection{Blade Angles}

With the basic blade shape chosen, the individual parameters had to be determined next. The basic methodology behind the design of the blade was that with inlet and outlet velocities and angles fixed by the required performance, the blade could then be shaped to deliver these values while minimizing losses. This method required an iterative process starting with an initial guess in order to converge on a reasonable blade design. This process is shown here in Figure 40.

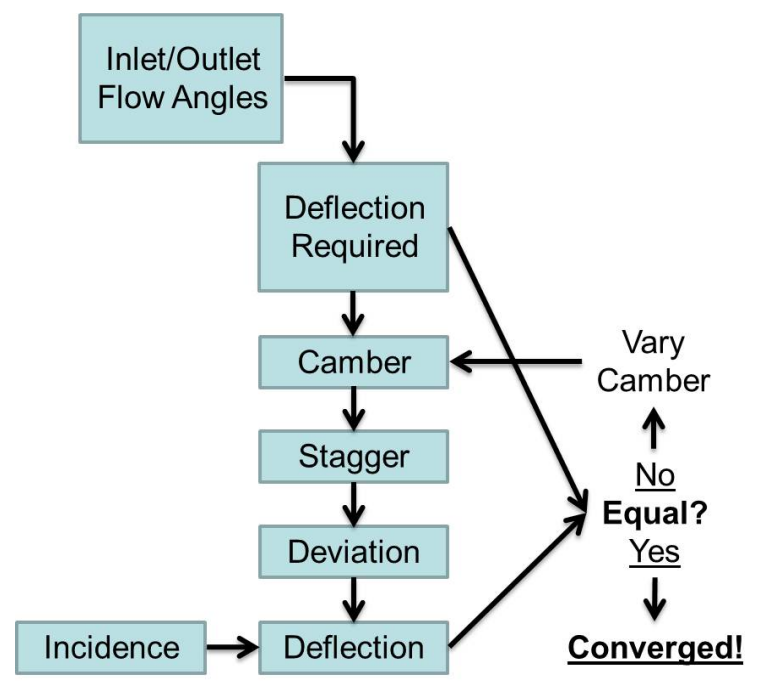

Figure 40. Blade Design Flowchart.

First, the flow inlet and outlet angles are used to calculate the required deflection angle. This calculation was shown previously in equation (4.7.6.3). This value provides the initial guess for the blade camber angle. With zero incidence and deviation, the camber angle equals the required deflection angle, but this is rarely the case, especially at off design conditions. Next the stagger angle is calculated assuming zero incidence. The choice of zero incidence is explained later.

$$
\xi=\beta_{2}-\frac{\theta}{2}
$$

Next, the deviation is computed using empirical data from The Low Speed Performance of Related Airfoils in Cascades by A D S. Carter ${ }^{x \times i i}$. In this report, Carter uses experimental data to predict the deviation angle based on camber, stagger, solidity, and his derived coefficient, referred to commonly as "Carter's Rule". Figure 41 from this report shows Carter's deviation coefficient $(m)$ versus blade stagger angle for blade sections with both parabolic arc and circular 
arc camber lines. Figure 42 shows the $3^{\text {rd }}$ order polynomial curve fits that were created for this data.

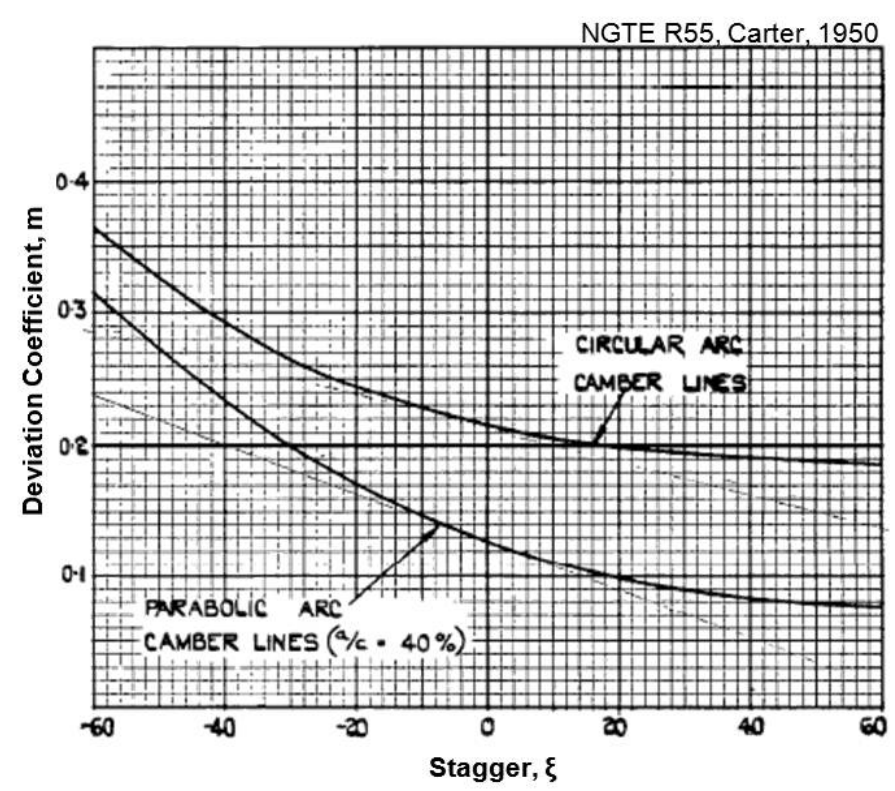

Figure 41. Carter's Deviation Coefficient vs. Stagger Angle.

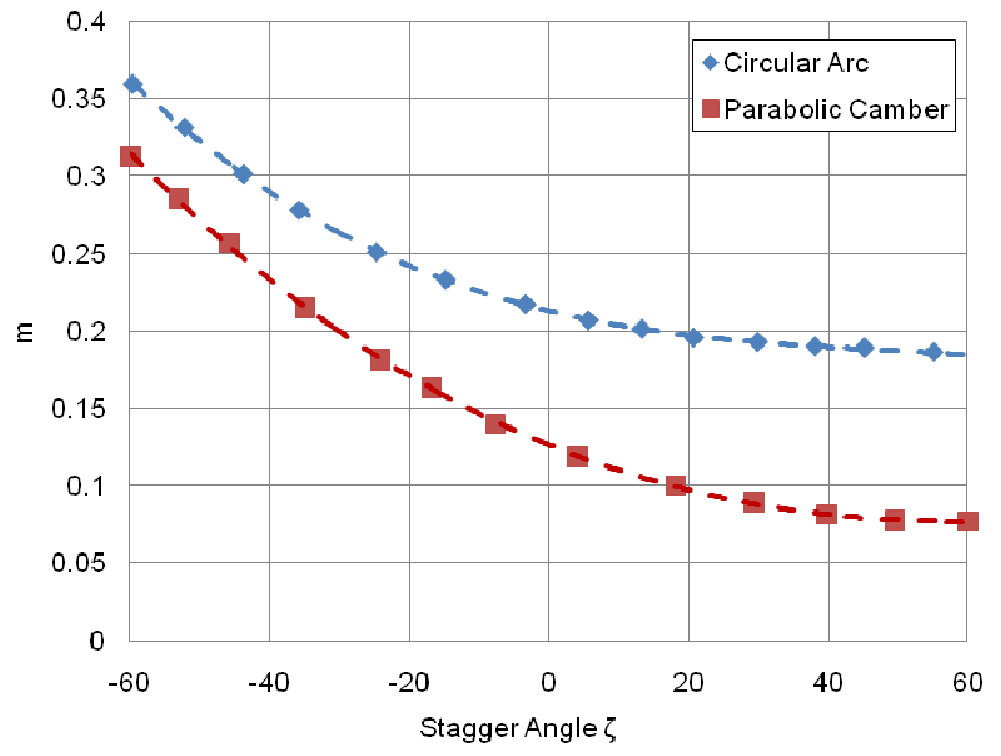

Figure 42. Curve Fits to Carter's Deviation Coefficient.

Using the deviation coefficient from curve fit, the deviation angle is estimated using the following equation created by Carter. 


$$
\delta=m \theta \sqrt{\frac{s}{c}}
$$

Finally, the resulting deflection when taking into consideration the incidence and deflection is easily calculated with the following equation.

$$
\varepsilon^{*}=\theta+i-\delta
$$

This deflection is then used as the new guess for the camber angle, and the loop iterates until the calculated deflection equals the required deflection. In most cases, the deviation angle will be a positive value, requiring that the blade camber angle be greater than the required deflection angle.

With the mean blade cross section designed, it was desirable to look at effect that tip Mach number and air inlet angle would have on the various blade geometries such as required deflection, camber, stagger, and deviation. Figure 43 through Figure 46 show these trades in an identical design space as the other 2D performance trades. Again, a typical design point is indicated with a red circle.

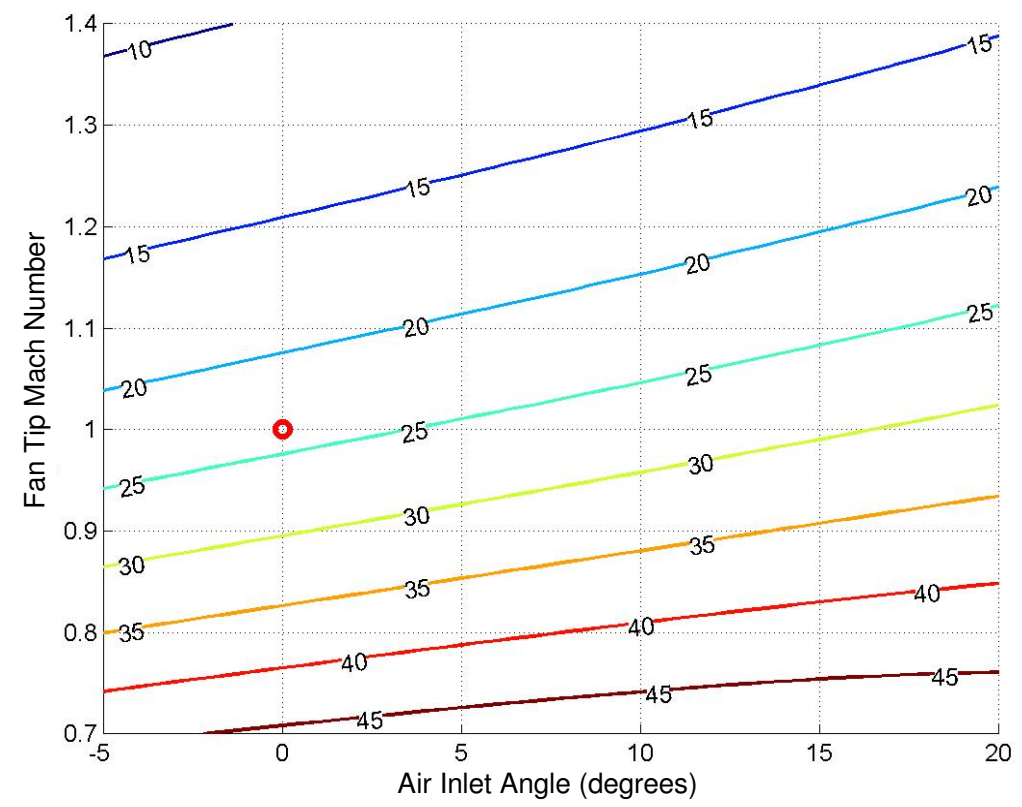

Figure 43. Required Fluid Deflection vs. Tip Mach Number and Air Inlet Angle. 


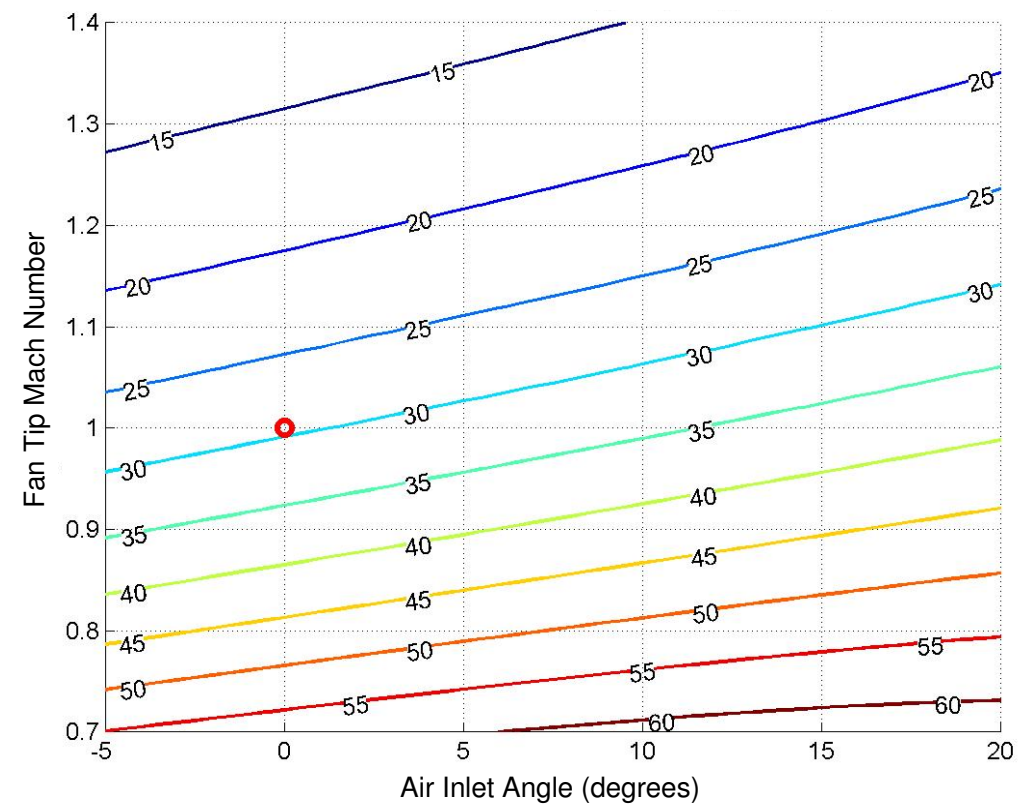

Figure 44. Blade Camber Angle vs. Tip Mach Number and Air Inlet Angle.

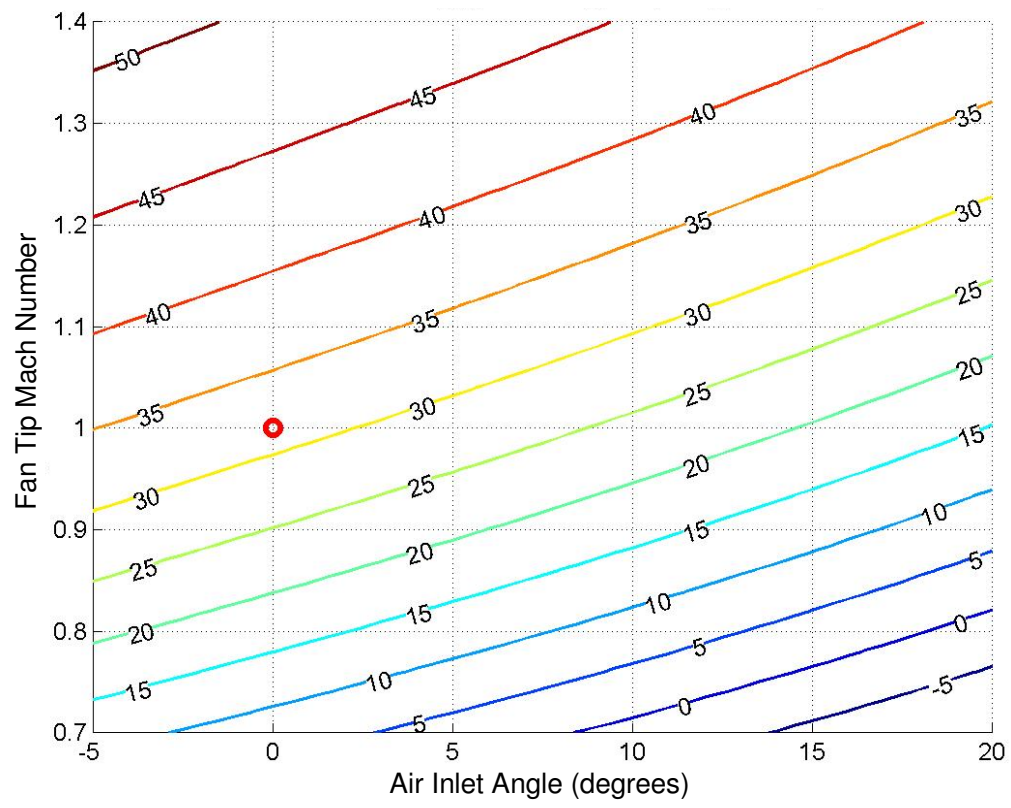

Figure 45.Blade Stagger Angle vs. Tip Mach Number and Air Inlet Angle. 


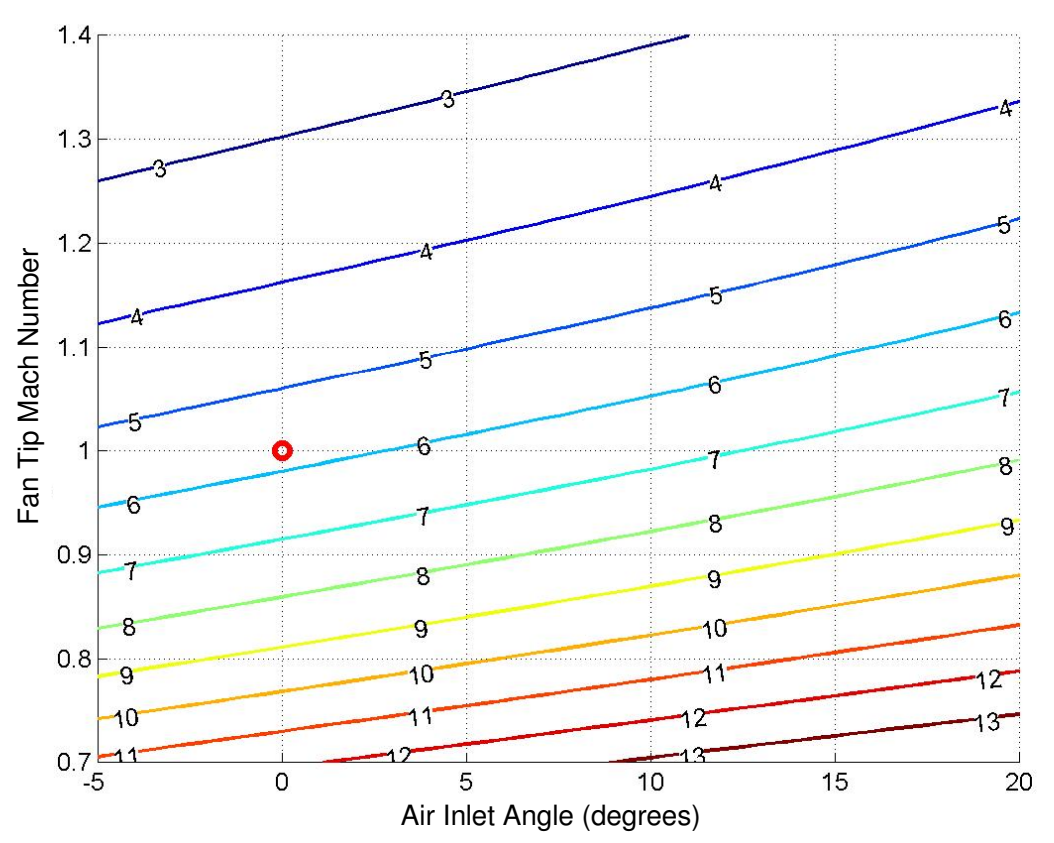

Figure 46. Deviation Angle vs. Tip Mach Number and Air Inlet Angle.

These figures, all showing roughly similar trends, reveal that higher air inlet angles require more "extreme" blade geometry. For example, by increasing the air inlet angle from $0^{\circ}$ to $20^{\circ}$, the required blade camber angle increases by about $10^{\circ}$. Alternatively, increases in tip Mach number tend to "flatten" out the blades. Increasing the tip Mach number from 1.0 to 1.4 can reduce the blade camber to about $12^{\circ}$. These variations in geometry are reasonable since the lower right hand corner of the figures lead towards an impulse rotor (large rotor turning angle), while the upper left hand corner leads to impulse stator (small rotor turning angle).

\subsubsection{Incidence Angle}

Earlier, it was stated that zero incidence is chosen to perform the 2D blade design. This assumption is common practice early in the process. However, various experimental results were researched to determine perhaps a more reasonable guess for the incidence angle. The first set of data in Figure 47 also came from Carter's research on transonic compressor blading. This figure plots both the deflection angle and incidence angle versus camber angle for a blade with a solidity of 1.0 and a relative outlet flow angle of $20^{\circ}$. 


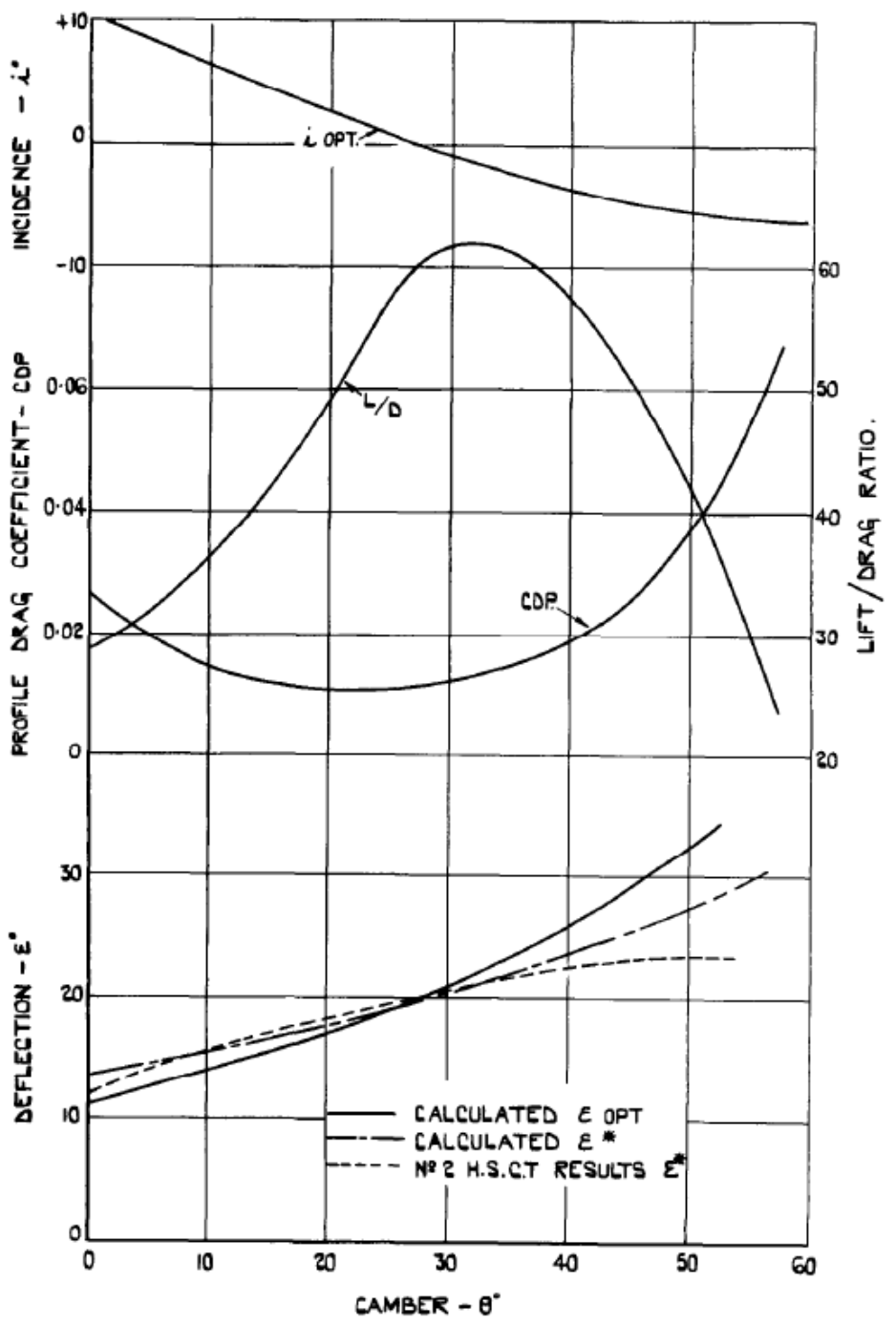

Figure 47. Blade Incidence, Drag, and Deflection vs. Camber Angle ${ }^{x \times i i}$.

This figure reveals that the maximum blade L/D occurs at an incidence angle of approximately $-2^{\circ}$, and that blade drag begins to rise rapidly after a camber angle of about $40^{\circ}$. In future iterations of HAPSS, the 2D blade design tool could include a curve fit of incidence angle versus camber angle such that it can be calculated inside the iterative loop. However, if the solidity or relative outlet flow angle were to change a significant amount, this data would no longer be valid. A similar but more comprehensive data set would be required for other fan blade designs. 
The second set of experimental results shown in Figure 48 came from Experimental Investigation of an Axial-Flow Compressor Inlet Stage Operating at Transonic Relative Inlet Mach Numbers by Schwenk, Lieblien, and Lewis ${ }^{x \text { xiii }}$.
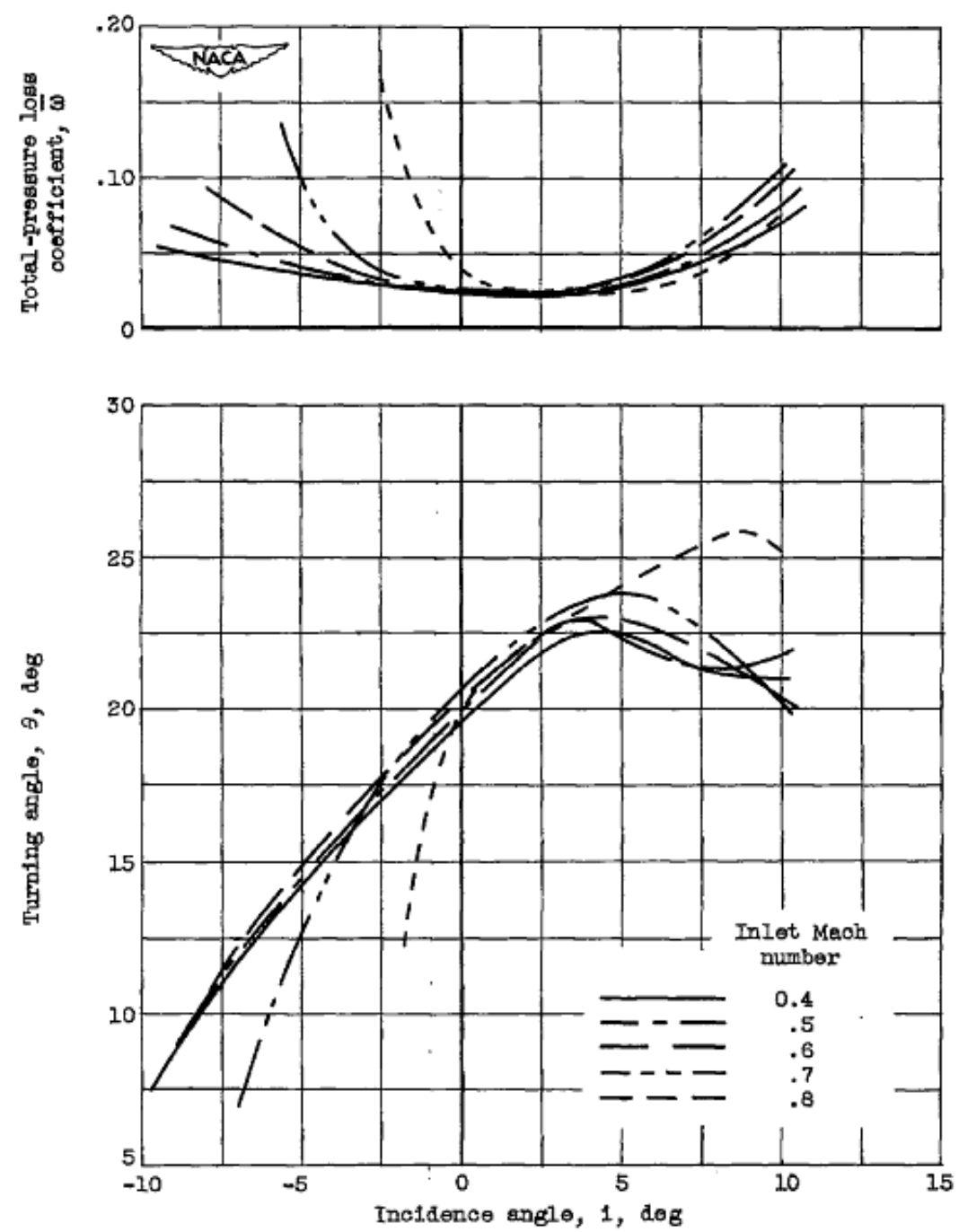

Figure 48. Total Pressure Loss and Deflection Angle vs. Incidence Angle ${ }^{x x i i}$.

This figure represents transonic blade testing for a compressor with a solidity of 1.33 , a relative inlet flow angle of $55^{\circ}$, a camber of $25^{\circ}$, a thickness to chord ratio of $10.5 \%$, and a tip speed of $1,000 \mathrm{ft} / \mathrm{s}$. According to this data, to minimize losses, an incidence angle of about $2.5^{\circ}$ is preferred for a very wide range of inlet Mach numbers. This data compared to the previous data shows how incidence angle can be affected by the rotor's other geometry. The incidence angle is also highly dependent on the rotor speed. "A tendency exists for the minimum-loss point to shift several degrees to higher values of incidence angle as the rotor tip speed is increased." "xiii In 
order to expand the blade design tool to a wider range of solidities, camber angles, etc, more data would be needed to formulate the proper curve fits.

\subsubsection{Validation}

In order to validate the 2D blade design, a study of a single stage compressor design was found. This study was names "Experimental Investigation of a Transonic Axial-Flow-Compressor Rotor with Double-Circular-Arc Airfoil Blade Sections" by Lewis, Schwenk, and Serovy ${ }^{x x i v}$. The purpose of this experiment was to provide valuable data for double-circular-arc airfoil sections used in high speed compressors. Figure 49 shows a schematic of the compressor that was used in the experiment.

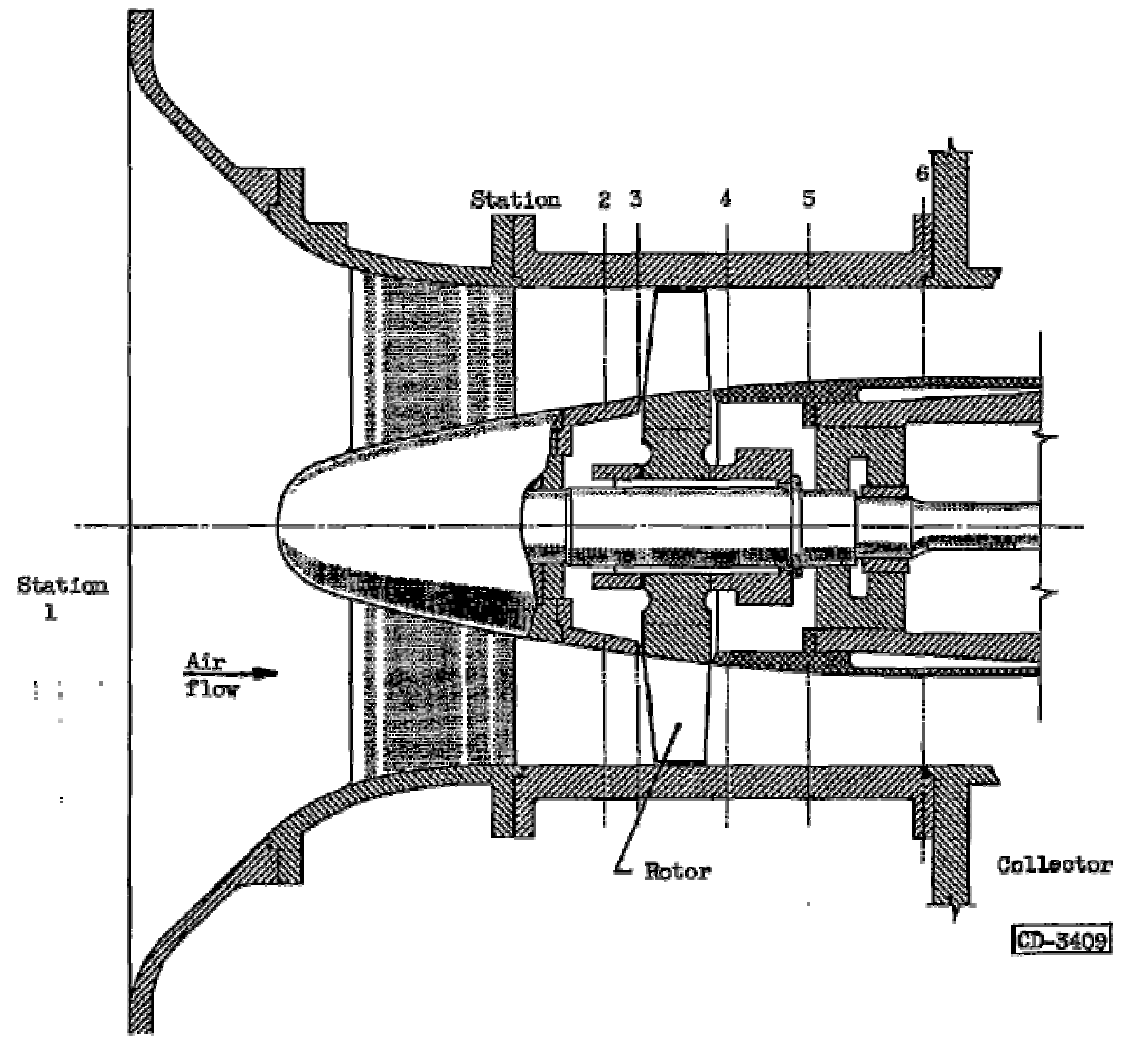

Figure 49. Schematic of Experimental Compressor Stage ${ }^{x x i v}$.

Table 6 shows the design variables that were used in this experiment. These values were inputted into the HAPSS code for both the 1D and 2D performance analyses in order to determine the similarities and differences between the HAPSS 2D results and this experiment. Table 7 and Table 7 show these results compared to the experiment. 
Table 6. 2D Fan Performance Validation Inputs.

\begin{tabular}{|l|c|c|}
\hline \multicolumn{1}{|c|}{ Input } & Units & Value \\
\hline Fan Diameter & in & 14.0 \\
\hline Fan pressure ratio & - & 1.34 \\
\hline Fan face Mach number & - & 0.60 \\
\hline Blade tip velocity & $\mathrm{ft} / \mathrm{s}$ & 1000 \\
\hline Hub-to-tip ratio & - & 0.5 \\
\hline Fan adiabatic efficiency & $\%$ & 93.0 \\
\hline Rotor solidity & - & 1.19 \\
\hline Incidence angle & $\circ$ & 3.5 \\
\hline Air Flow inlet angle & $\circ$ & 0.0 \\
\hline
\end{tabular}

Table 7. 2D Fan Performance Validation Outputs.

\begin{tabular}{|l|c|c|c|}
\hline \multicolumn{1}{|c|}{ Output } & Units & HAPSS & Experimental \\
\hline Relative air flow inlet angle & $\%$ & 49.2 & 45.5 \\
\hline Relative air flow outlet angle & $\circ$ & 29.1 & 23.7 \\
\hline Deflection angle & $\circ$ & 20.1 & 21.8 \\
\hline Inlet relative Mach number & - & 0.89 & 0.82 \\
\hline Tip relative Mach number & - & 1.07 & 1.10 \\
\hline Deviation angle & $\circ$ & 4.4 & 4.5 \\
\hline Mass flow per unit area & $\left(\mathrm{lb}_{\mathrm{m}} / \mathrm{s}\right) / \mathrm{ft}^{2}$ & 30.9 & 30.5 \\
\hline
\end{tabular}

In this validation, the fan polytropic efficiency in HAPSS was adjusted until the adiabatic efficiency was $93 \%$. This resulted in a polytropic efficiency of $93.29 \%$. The fan tip Mach number was also adjusted to 0.927 to achieve the correct blade tip velocity. Mode 8 of the $1 \mathrm{D}$ fan analysis was utilized since its inputs are fan diameter, fan face Mach number, and fan pressure ratio.

The 2D results from HAPSS match those from this experiment very well. HAPSS currently assumed an incidence angle of $0^{\circ}$ while the experiment had a design incidence angle of $3.5^{\circ}$ at the mean fan diameter. Despite this difference, the results are strikingly similar.

The fan in this experiment used blades with a thickness to chord ratio of $8 \%$ and $5 \%$ at the hub and tip, respectively. This ratio varied linearly from hub to tip, giving a $6.5 \%$ thickness to chord at the mean fan diameter. At this time however the thickness to chord ratio does not play a role in the performance of the fan. 


\subsection{Electrical System Analysis}

The electrical system is considered next in the on-design analysis. The electrical system is what connects the thrust producer to the power producer in a series hybrid system. In HAPSS the electrical system includes the motors which drive the fans, the controllers, and the generators which generate the electrical power. Figure 50 illustrates the generic pure series hybrid propulsion system modeled by HAPSS with the electrical system boxed with the dashed gray line.

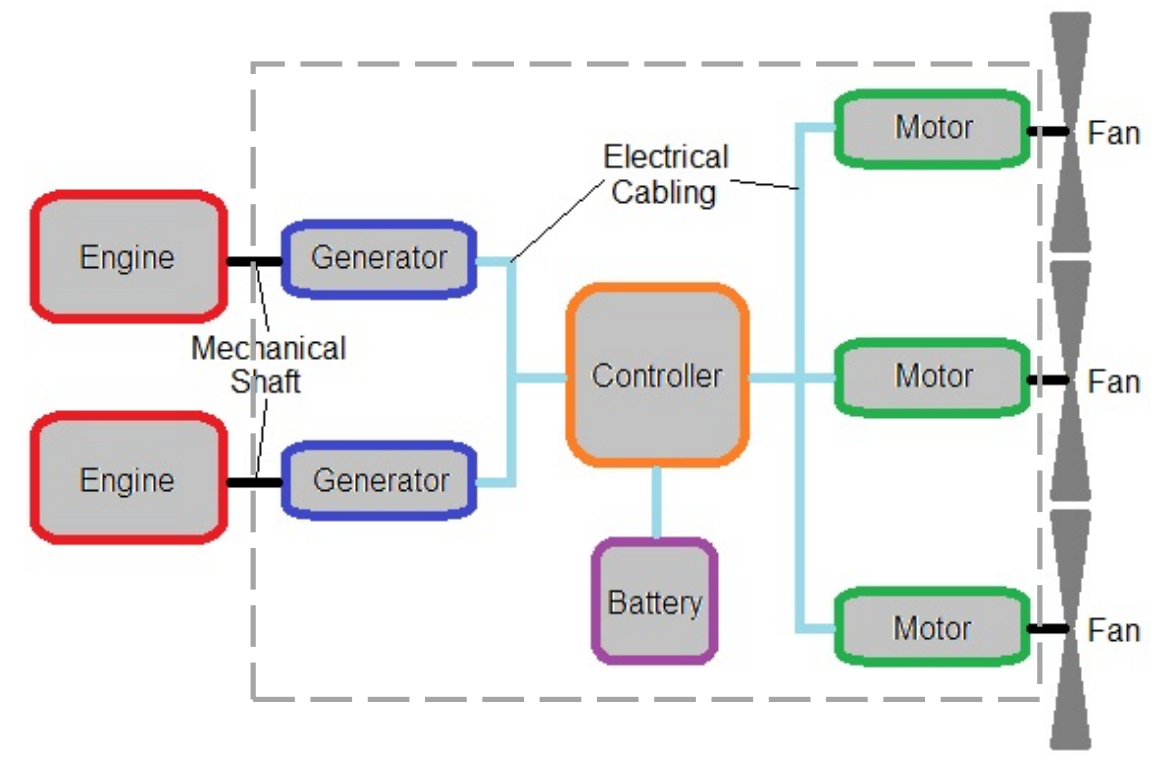

Figure 50. Generic Pure Series Hybrid System Diagram, Electrical System Highlighted.

While this figure shows three motors and two generators, HAPSS can model any combination of these components. For simplification purposes however, the controller in the system is modeled as a single unit controlling all of power flow. The battery is modeled as a single source of auxiliary power. HAPSS models the electrical system as a simple power management device. Future iterations of the code will give considerations for voltage, current, resistance, and even frequency.

The electrical system module in HAPSS begins by checking all of the inputs to the module. Such inputs include motor and generator design points, motor, generator, battery, and controller efficiencies, generator rotational speed and diameter, power provided by the battery at the design point, and more. As will be explained in the following sections, the motor and generator efficiencies (and other parameters) can be fixed or supplied by functions. 


\subsubsection{Design Points}

Like the aircraft, the motors and generators have a design point at which they will operate during the on-design analysis. This design point is defined as some percentage of the machine's absolute maximum speed and absolute maximum torque. For example, the machine's design point could be $50 \%$ of maximum speed and $50 \%$ of maximum torque. In the analysis, the electric machine's design point occurs at the same design point as the aircraft.

In this iteration of HAPSS, it is assumed that the motor's speed and torque at the design point equal that of the fan (in other words there is no gearbox). Future work on this code will include the option for gearboxes. The maximum speed and maximum torque of the motor and generator can then be calculated.

$$
\begin{aligned}
& N_{\text {motor, } \text { max }}=\frac{N_{\text {fan }}}{\Lambda_{N, \text { motor }}} \\
& T_{\text {motor, } \text { max }}=\frac{T_{\text {fan }}}{\Lambda_{T, \text { motor }}}
\end{aligned}
$$

where $\Lambda_{N \text {,motor }}$ and $\Lambda_{T \text {,motor }}$ are the speed and torque fractions that define the design point, respectively; they must be values between zero and one.

The generator on the other hand is driven by the turboshaft engine. Without detailed cycle analysis and sizing, the output speed of the turboshaft engine is unknown. This speed also depends on the use of a power turbine and/or a gearbox. Therefore, it is the user's responsibility to input the generator's speed at the design point. The maximum speed of the generator is then calculated as follows.

$$
N_{\text {gen,max }}=\frac{N_{\text {gen }}}{\Lambda_{N, \text { gen }}}
$$

The torque of the generator cannot be calculated until its output power in known; the output power of the generator depends on the electrical distribution system. This power is calculated using equation 4.8.3.X described in section 4.8.3. With this power, the generator torque and maximum torque can be calculated.

$$
\begin{gathered}
T_{\text {gen }}=\frac{33000 * P w r_{\text {gen }, \text { out }}}{2 \pi * N_{\text {gen }}} \\
T_{\text {gen }, \text { max }}=\frac{T_{\text {gen }}}{\Lambda_{T, \text { gen }}}
\end{gathered}
$$




\subsubsection{Efficiencies}

Next, efficiency of the motor and generator must be considered. These efficiencies can be either fixed or calculated using a user-created function. If the machine's efficiency is defined using a function, this function must output the efficiency using the design point of the machine as an input. In the simplest sense, the motor efficiency is defined as the output power divided by the input power. The efficiency data for most electric machines is provided as a function of output torque and speed in contour map form. Figure 52 shows an HVH250-090-SOM motor produced by Remy International while Figure 52 shows this motor's efficiency map.

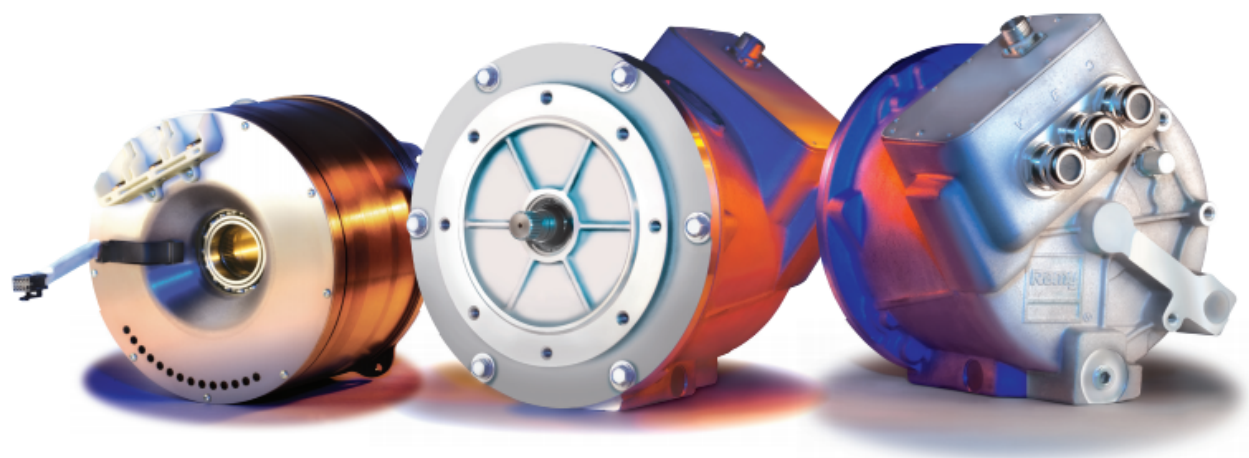

Figure 51. Remy HVH250-090-SOM ${ }^{\mathrm{xxv}}$.

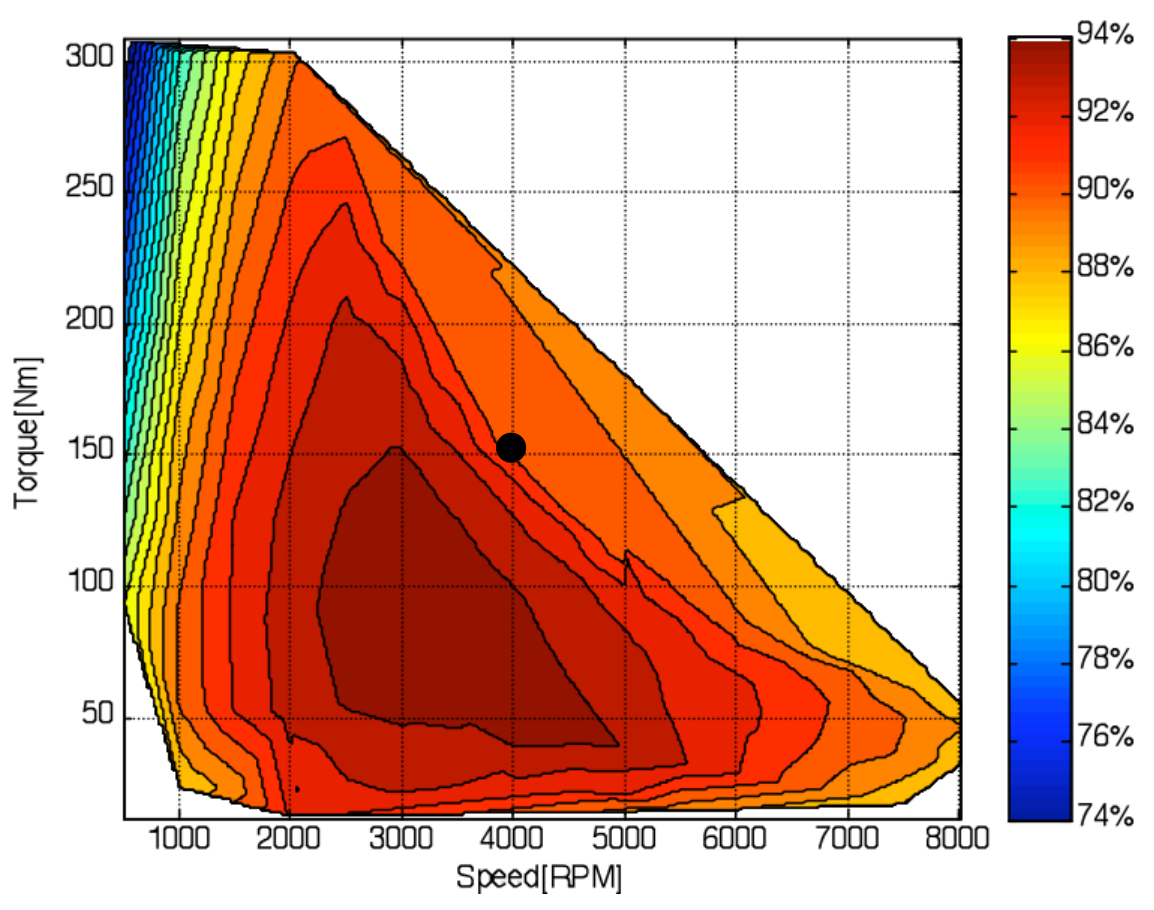

Figure 52. Remy HVH250-090-SOM Efficiency Map ${ }^{\mathrm{xxv}}$. 
In this figure, the black dot represents an example design point, in this case $\Lambda_{N}=50 \%$ and $\Lambda_{T}=50 \%$, which gives an efficiency of approximately $90 \%$. The user of HAPSS can create a function that defines this efficiency map as a function of the design point. The efficiency map must be normalized such that is can be scaled to represent any size motor while preserving the valuable interior trends.

The user may choose to utilize the same efficiency map for both the motor and generator, or use different maps. Likewise, the user could input a constant efficiency for the motor, and use a function to calculate the generator efficiency, or vice versa. Each motor must use the same fixed efficiency or function, same with the generators.

The controller and battery efficiency is fixed by the user and does not have the option to be calculated using a function. After some research, it was apparent a controller's efficiency varies little during operation. Efficiencies between $95 \%$ and $98 \%$ are common, and $99 \%$ may be achievable in the future with cryogenic cooling. Generally, more efficient controllers tend to be heavier. Battery efficiency can be assumed to be $\sim 99 \%$.

The system efficiency is the product of the generator, controller, and motor efficiencies and is useful for comparison with traditional turbofan performance. The battery efficiency is not included in this because it is modeled source of secondary power and its performance does not affect the flow of power from the power producer to the thrust producer. The following equation shows the calculation of electrical system efficiency.

$$
\eta_{\text {system }}=\eta_{\text {gen }} \eta_{\text {cont }} \eta_{\text {motor }}
$$

This efficiency offers a good indication of how much power is being lost between the power producer and the thrust producer. In a turbofan engine the power producer is connected to the thrust producer with a simple hollow shaft incurring minimal losses. In a hybrid electric system however, the losses would typically range from 10\%-20\% for conventional "warm" technology, and $1 \%-5 \%$ for cryogenically cooled machines.

\subsubsection{Power Distribution}

The core of the on-design electrical system analysis is determining the power distribution between the electrical components. In this analysis, the fans have a specified power required as 
calculated in the 1D fan performance study. The primary purpose here is to determine the amount of power required at the input of the generators (output of turboshaft engines) such that the fans receive their required power at the design point. And in this process, the size and other performance aspects of the various components is calculated.

The diagram in Figure 53 shows the components of the electrical system and how the power flow is modeled between them.

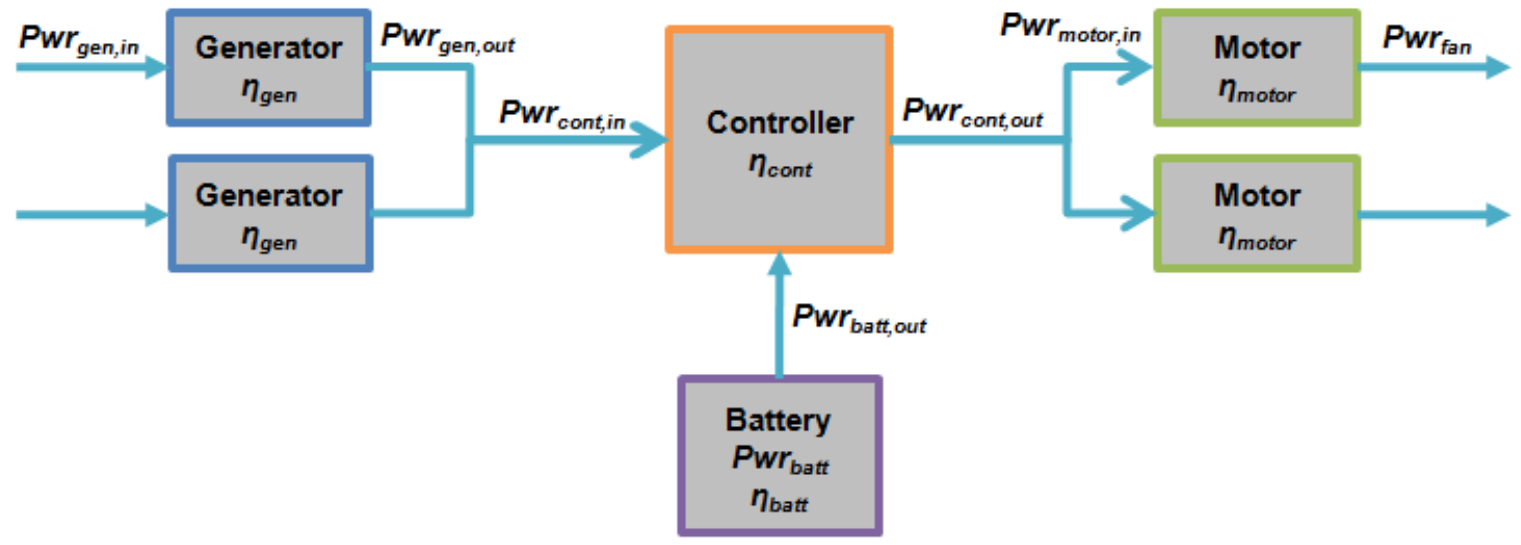

Figure 53. Electrical System Diagram, Battery Discharging.

In this figure, the blue arrows indicate the flow of power through the system. This illustration shows two generators and two motors, but HAPSS is able to model any combinations of motors and generators. In this example, the battery is discharging, or providing supplemental power to the system. Power produced in the generators and power released from the battery both enter the controller, where it is summed and delivered to the motors. As power flows through the system, various inefficiencies reduce the total power available to the motors.

The known variables in this diagram is the power supplied to the fan, the power supplied by the battery (inputted by the user), and the efficiency of each component. The following equations use the nomenclature in the figure above. The power required by each motor and the total power outputted by the controller to all of the motors are calculated first.

$$
\begin{gathered}
P w r_{\text {motor, }, \text { in }}=\frac{P w r_{f a n}}{\eta_{\text {motor }}} \\
P w r_{\text {cont }, \text { out }}=P w r_{\text {motor }, \text { in }} n_{\text {fan }}
\end{gathered}
$$

where $\eta_{\text {motor }}$ is the motor efficiency (either fixed or calculated using the efficiency map at the design point), $P w r_{f a n}$ is the power required by the fan calculated during the $1 \mathrm{D}$ fan performance 
analysis, and $n_{\text {fan }}$ is the number of propulsive fans. Next, the battery output power is calculated. In the case of the battery discharging, this output power will be positive.

$$
P w r_{\text {batt }, o u t}=P w r_{\text {batt }} \eta_{\text {batt }}
$$

where $P w r_{\text {batt }}$ is the power the battery can supply and $\eta_{\text {batt }}$ is battery's efficiency. $P w r_{\text {batt }}$ is inputted by the user and is positive if the battery is discharging and negative if charging. Next, the total input power to the controller from all of generators can be calculated.

$$
P w r_{\text {cont }, \text { in }}=\left[P w r_{\text {cont }, \text { out }}-\left(P w r_{\text {batt }, \text { out }} \eta_{\text {cont }}\right)\right] \frac{1}{\eta_{\text {cont }}}
$$

where $\eta_{\text {cont }}$ is the efficiency of the controller inputted by the user. Finally, the output and input power of each generator is calculated as follows.

$$
\begin{aligned}
& P w r_{\text {gen }, \text { out }}=\frac{P w r_{\text {cont }, \text { in }}}{n_{\text {gen }}} \\
& P w r_{\text {gen,in }}=\frac{P w r_{\text {gen,out }}}{\eta_{\text {gen }}}
\end{aligned}
$$

where $\eta_{g e n}$ is the generator efficiency (either fixed or calculated using the efficiency map at the design point) and $n_{g e n}$ is the number of generators. The generator input power will be used to determine the size of the turboshaft engines.

The previous calculations were for the situation in which the battery was discharging, supplying power to the system. The calculations vary to some degree if the battery is charging, taking power away from the system. The following system diagram in Figure 54 is similar to that in Figure 53, but the power is shown flowing into the battery for charging.

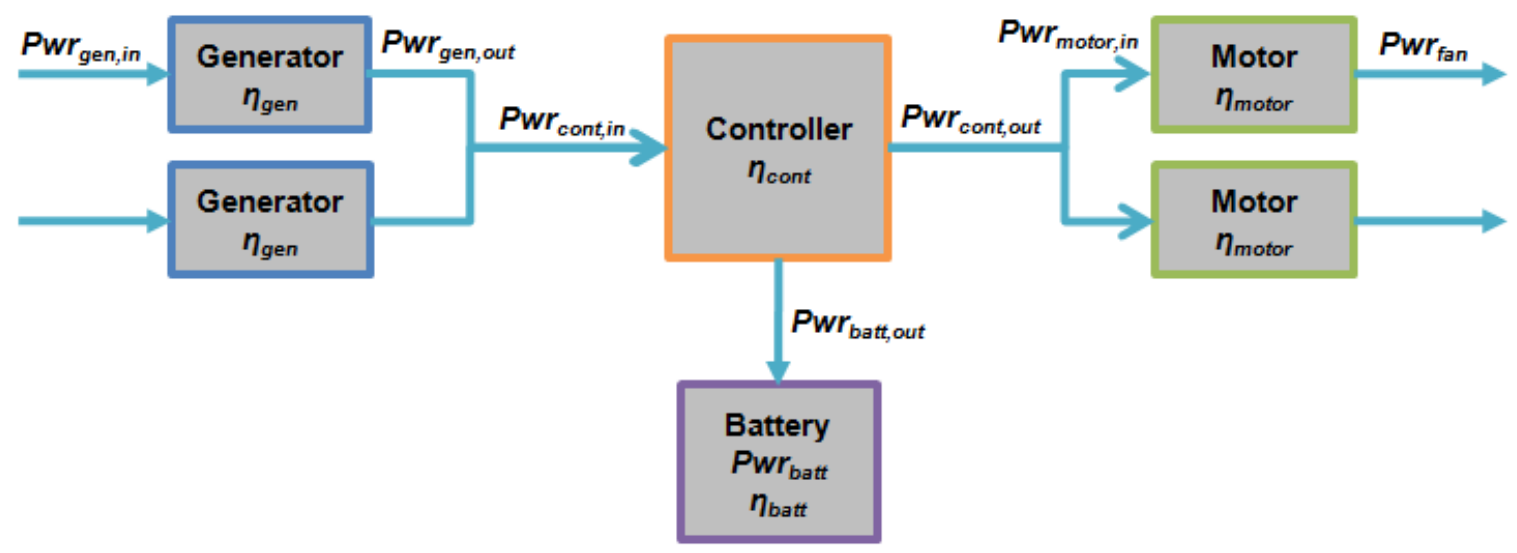

Figure 54. Electrical System Diagram, Battery Charging. 
The first step in this case is to calculate the battery output power, which will be negative since the battery is absorbing power for charging.

$$
P w r_{\text {batt }, \text { out }}=\frac{P w r_{\text {batt }}}{\eta_{\text {batt }}}
$$

where $P w r_{\text {batt,out }}$ is the power at the terminals of the battery and $P w r_{\text {batt }}$ is the power that the battery cells actually receive after inefficiencies in the battery itself. The controller output power to all of the motors is calculated in the same manner. Thus, the power inputted to the controller from all of the generators can be calculated as follows.

$$
P w r_{\text {cont }, \text { in }}=\left(P w r_{\text {cont }, \text { out }}-P w r_{\text {batt }, \text { out }}\right) \frac{1}{\eta_{\text {cont }}}
$$

The input and output power of each generator is calculated the same as before.

\subsubsection{Performance}

With the power and torque of the motors and generators known, more steps are taken to determine if these machines are operating within their limitations. Thus far, it is only known that these machines are operating below their maximum speed and maximum torque. However, electric motors and generators can be defined with performance curves which reveal their maximum power and torque with respect to speed. Figure 55 and Figure 56 show the torque and power curves for the Remy HVH250-090-SOM motor. 


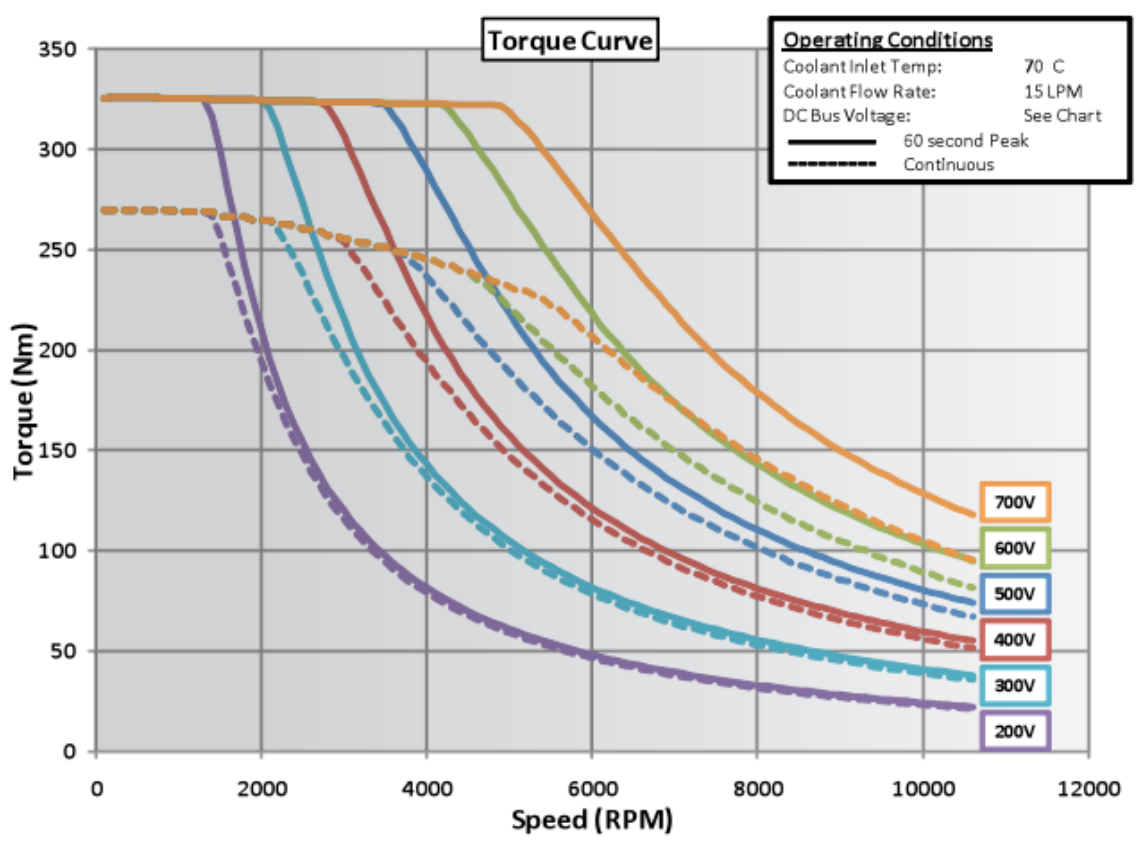

Figure 55. Remy HVH250-090-SOM Torque Curves ${ }^{\mathrm{xxv}}$.

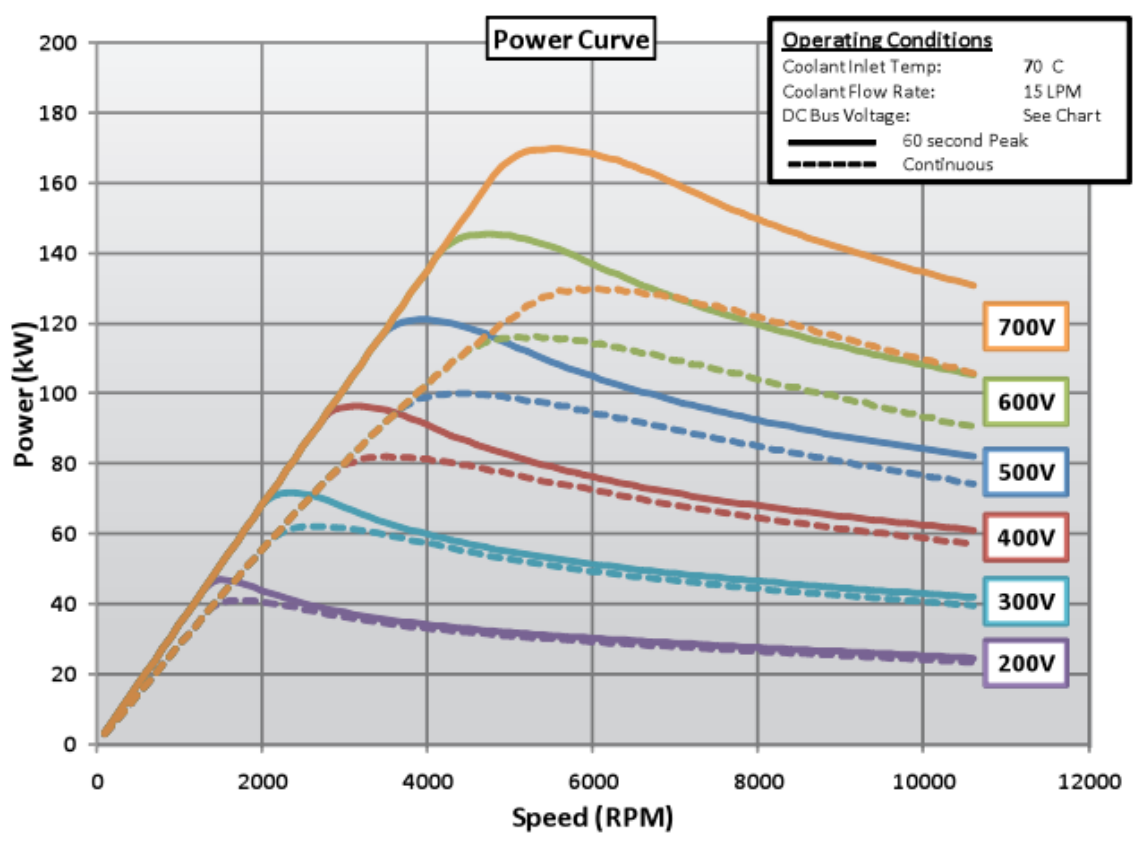

Figure 56. Remy HVH250-090-SOM Power Curves ${ }^{\mathrm{xxv}}$.

In these figures, the torque and power of the motor is plotted with respect to speed, input voltage, and whether or not the motor is operating in continuous or 60 second peak mode. The 60 second peak curves indicate that the motor can operate at that point for only 60 second before overheating. The continuous curves indicate that the motor can operate at that point indefinitely as long as there is sufficient cooling. 
For use with HAPSS, the user must create a MATLAB function that contains this data. Since voltage is not part of this version of HAPSS, any of the voltage lines could be used, although the voltage does affect the behavior of these curves.

The current iteration of HAPSS has a function that defines the Remy $700 \mathrm{~V}$ torque and power curves for both peak and continuous power. As with the efficiency map, these values are normalized such that the curves can be scaled to fit the size of the motor and generator. This function takes the operating point of the machine as an input and outputs the operating point's percentage of peak and continuous torque at that speed. The function will also output the operating point of the machine's maximum output power. Figure 57 offers a visualization of the output of this function.

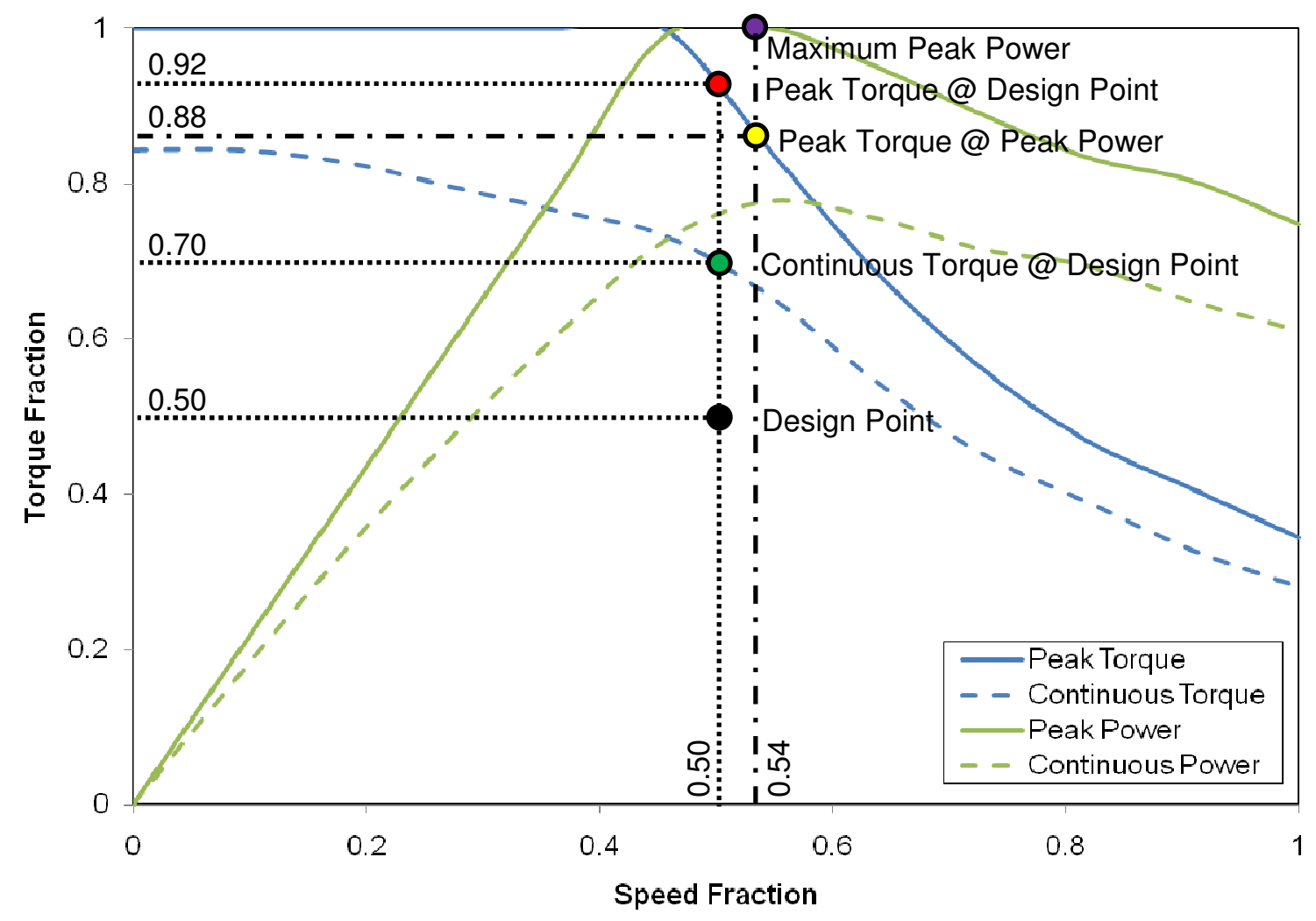

Figure 57. Normalized Torque and Power Curves.

In this figure, the blue lines are the $700 \mathrm{~V}$ torque curves from the Remy data, while the green lines are the $700 \mathrm{~V}$ power curves. The dashed lines are continuous while the solid lines are 60 second peak. The Remy data has been normalized in this figure. 
The black dot represents the chosen example design point of the machine; in this case $50 \%$ of maximum speed and $50 \%$ of maximum torque. The green and red dots are the maximum continuous and maximum peak torque at this speed, respectively. The function outputs the torque fraction of the design point over the torque fraction of the green and red dots. In this example, the machine would be operating at $0.50 / 0.70=71 \%$ of continuous torque and $0.50 / 0.92=54 \%$ of peak torque. These values are useful as they indicate whether or not the machine is operating within its continuous and peak limits.

The yellow dot indicates the peak torque fraction of the machine at the speed at which the machine produces the most power (the maximum peak power point it shown with the purple dot). This knowledge is useful for calculating the maximum peak power of the machine which will be used to estimate its weight.

\subsubsection{Weight}

When trying to predict the physical dimensions of the electric motors and generators, it became apparent that relevant sizing equations and methodologies are far and few between. Thus, empirical data and curve-fit equations for existing machines had to be leveraged.

The current version of HAPSS estimates the weight of the motors and generators by using a curve-fit of empirical data for approximately 100 modern high power electric motors and generators. This curve fit gives the machine's weight as a function of its maximum peak output power (the purple dot in Figure 57). This data was acquired by researching the products from dozens of motor manufactures such as Remy, EVO Electric, UQM, Tesla, and YASA to name a few. Figure 58 shows weight versus maximum peak power data for many modern electric motors and generators. 


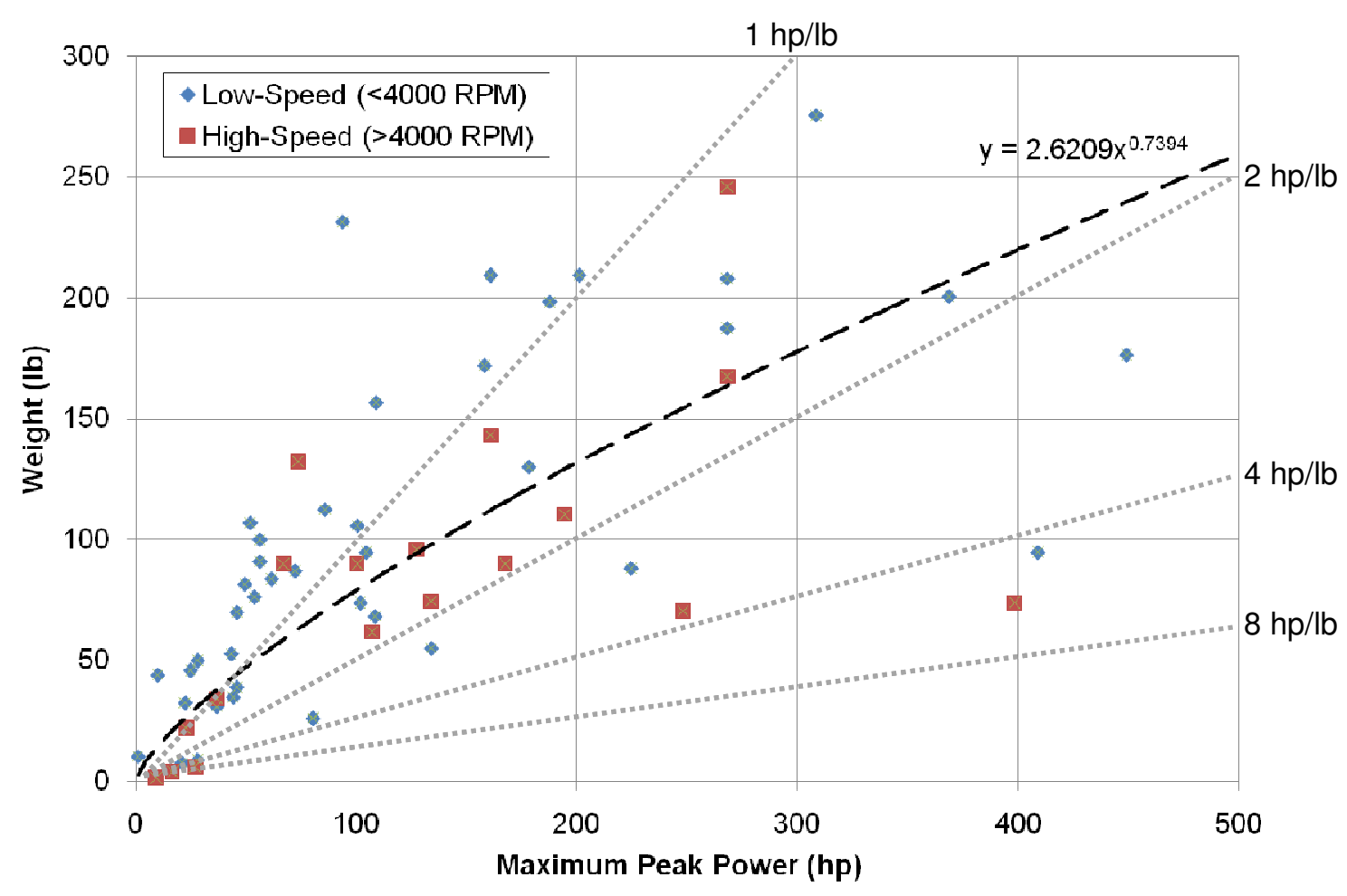

Figure 58. Motor/Generator Weight versus Maximum Peak Output Power.

In this figure the low-speed motors were defined as having a base speed under 4000 RPM, while high-speed motors have a base speed over 4000 RPM. This dividing speed was chosen arbitrarily and has no effect on the results. The dotted gray lines are lines of constant power density. The dashed black line is a curve-fit using a power law equation. This type of curve-fit was chosen because it can reasonably estimate the increase in power density as the machine gets larger.

The researched machines only had output powers up to about $450 \mathrm{hp}$, and varied greatly in power density. Machines with higher output power tended to be heavy industrial cast iron units optimized for cost, reliability, and efficiency rather than weight, and had very poor power densities. Today's vehicles (both automobiles and aircraft) requiring lightweight power-dense motors also tend to have relatively low power requirements. To date there has been no known need for lightweight electric motors and generators for large aircraft use; this technology must be developed.

The curve-fit equation resulting from this data was used in HAPSS to estimate the weight of the electric motors and generators. Future iterations of HAPSS will investigate alternative 
estimations such as weight versus maximum peak torque or using a constant power density or torque density inputted by the user.

The controller weight was estimated by researching machine plus controller combinations. It was found that the controller, on average, weighs approximately $30 \%$ of the machine that it controls. Without performing detailed controller design it is difficult to predict the controller weight with more fidelity. With a weight-conscious design required for an aircraft, the $30 \%$ would likely overestimate the controller weight. This weight estimation does not assume a single central controller as illustrated in Figure 53 and Figure 54, but rather assumes that each motor and generator has its own controller.

\subsubsection{Dimensions}

Similar to the motor/generator weight, the dimensions of these machines had to be estimated using empirical data. Unlike the weight however, the diameter and length of the machine can be tailored to suit the vehicle. In electric motor and generator design it can be assumed that the machine's dimensions scale with output torque as follows.

$$
\begin{gathered}
\text { Torque }^{2} \alpha \text { Diameter } \\
\text { Torque } \alpha \text { Length }
\end{gathered}
$$

These sizing relations indicate that the machine's torque is proportional to its volume, and that increasing diameter will yield more torque than increasing length. When the diameter is increased, not only does the surface area between the rotor and stator increase, the moment arm of the force is increased as well. Increasing the length only adds surface area while the moment arm remains the same.

HAPSS currently utilizes a sizing function which inputs the machine's diameter and maximum torque and outputs the machine's length. This is done by using a fixed volumetric torque density in $\mathrm{ft}-\mathrm{lb}_{\mathrm{f}} / \mathrm{ft}^{3}$. This volumetric torque density is a value chosen by comparing modern

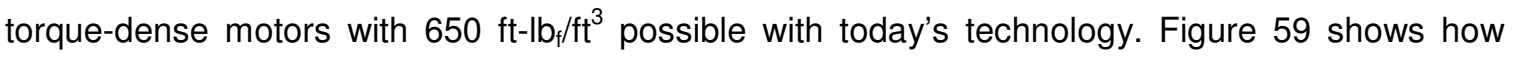
motor length changes with diameter and maximum peak torque for a machine with a volumetric torque density of $650 \mathrm{ft}-\mathrm{lb}_{\mathrm{f}} / \mathrm{ft}^{3}$. 


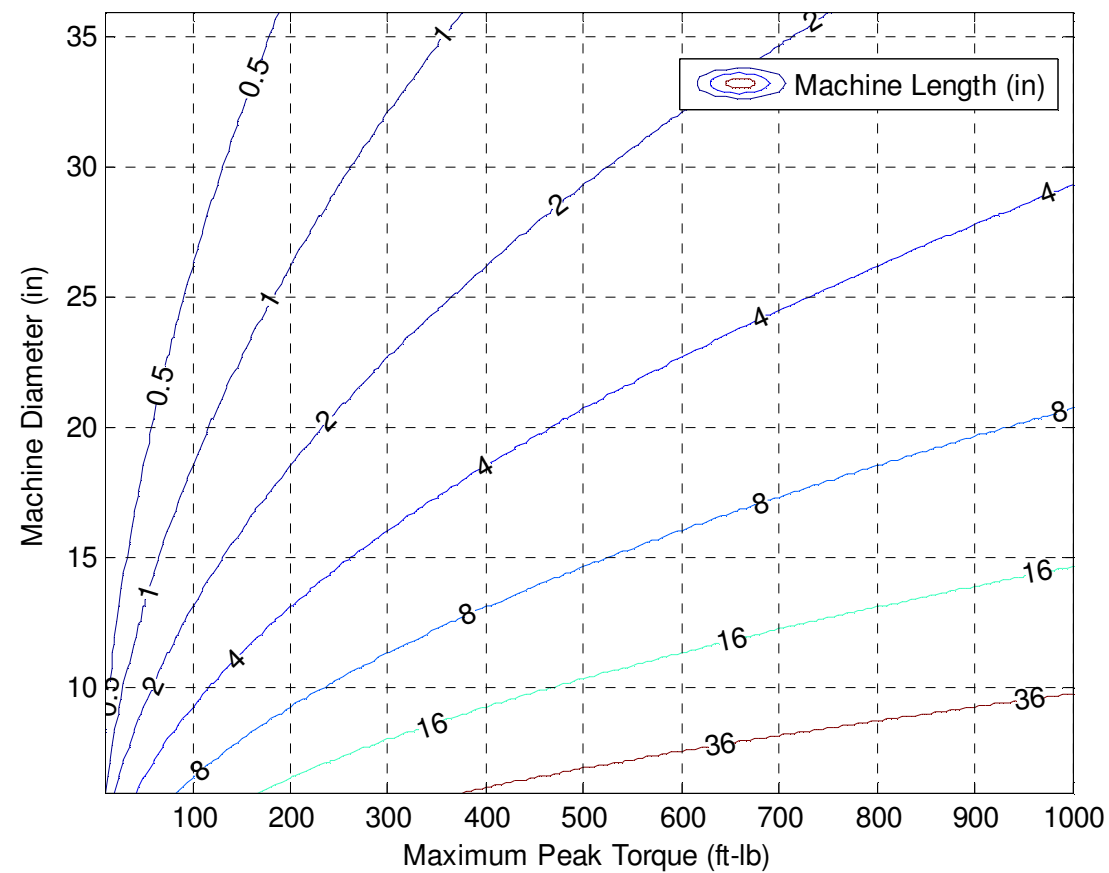

Figure 59. Motor/Generator Length versus Output Torque and Diameter for $650 \mathrm{ft}-\mathrm{lb} / \mathrm{ft}^{3}$.

This figure reveals that adding diameter to a motor or generator can greatly reduce its length while achieving the same torque output. This method is a simple approximation of a machine's dimensions for preliminary analyses. More accurate dimensions would require a detailed design study of the machine.

\subsubsection{Heating}

A major drawback of using electrical machinery is the introduction of inefficiencies which for the most part are converted directly into heat. Today's best conventional motors can only achieve efficiencies up to about 95\%. Therefore as an example, a 95\% efficient $1000 \mathrm{hp}$ motor must be able to dissipate $50 \mathrm{hp}$ of heat. As a vehicle designer interested in a hybrid propulsion system, cooling the electrical components would be a primary concern.

With this in mind, the electrical module of HAPSS calculates the amount of heat that each component creates. This information will give the designer valuable information for notionally sizing a cooling system or systems. The calculation of the heat loss is simple; a certain amount of power enters the machine and due to inefficiencies, slightly less power exits the machine. The power lost within the machine can be assumed to be converted completely to heat (although a 
negligible amount is also converted to vibrations and noise). The following equation shows the heat transfer rate for the generator in BTU/s.

$$
q_{\text {gen }}=\left(P w r_{\text {gen,in }}-P w r_{\text {gen,out }}\right) \frac{I}{J}
$$

In this equation, the power into and out of the generator are in horsepower, and the conversion factors I and $J$ are used to convert horsepower to BTU/s. The motor heat transfer rate is calculated in a similar fashion.

$$
q_{\text {motor }}=\left(P w r_{\text {motor, in }}-P w r_{\text {fan }}\right) \frac{I}{J}
$$

The heat transfer rate of the controller is calculated similarly, but also depends on the power flowing into or out of the battery.

$$
q_{\text {cont }}=\left(P w r_{\text {cont }, \text { in }}+P w r_{\text {batt }, \text { out }}-P w r_{\text {cont }, \text { out }}\right) \frac{I}{J}
$$

In this equation, the battery output power $P w r_{\text {batt,out }}$ is positive when discharging (supplying power to the system) and negative when charging (taking power from the system). Because HAPSS models the controller as a single central controller for the entire system, its heat transfer rate will usually be significantly higher than the other components.

\subsection{Engine Analysis}

The final piece of the on-design analysis is the consideration of the power producer, which in the TeDP case takes the form of a turboshaft engine. Similar to the motors and generators, the performance of turboshaft engine is provided in a performance map form. Future iterations of HAPSS will include a detailed cycle analysis module to better predict engine performance and to allow the user to modify the engine cycle.

\subsubsection{Power Lapse and PSFC}

This iteration of HAPSS requires the user to supply the performance of the engine in the form of a function. The inputs to the function are flight Mach number, altitude, and throttle setting. The output is the engine's power lapse (percent of sea level static maximum power) and PSFC in $\mathrm{lb}_{\mathrm{m}} /(\mathrm{hp}-\mathrm{hr})$. These values are not dependent on the output power of the engine and can thus be applied to any size turboshaft (similar to the normalization of the motor/generator data). 
It is common for engine manufacturers to publish power and fuel flow data at maximum throttle, however finding data that also varies with speed, altitude, and throttle can be more challenging. Figure 60 (same as Figure 9) shows a performance map for a Honeywell TPE331-10 turboprop engine. This data has already been transformed into a MATLAB function for use in HAPSS.

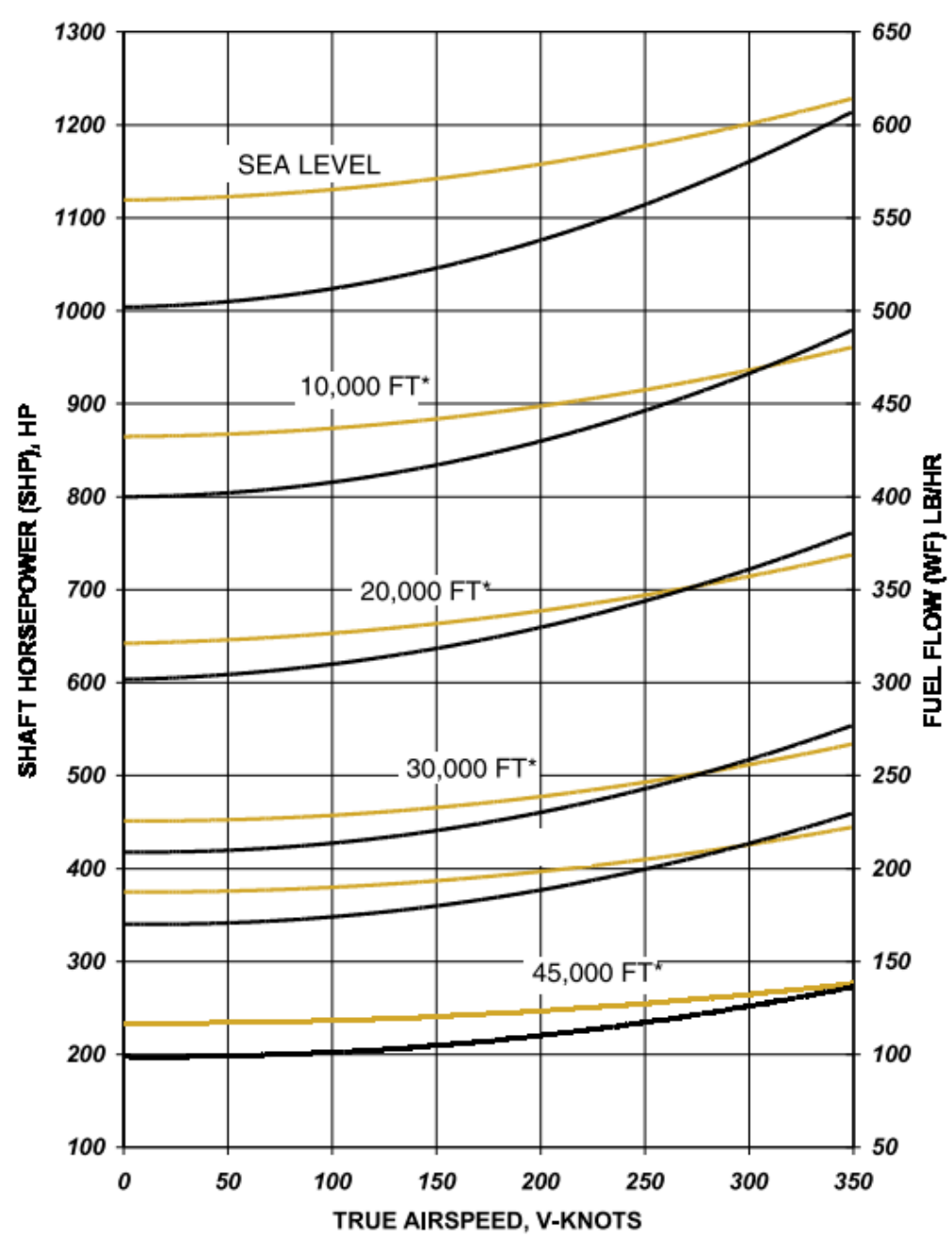

Figure 60. Honeywell TPE331-10 Turboprop Performance Map ${ }^{\mathrm{v}}$.

This chart is an example of the data the user can convert into a chart for use with HAPSS. In this chart, the power in black lines and fuel flow in orange lines are shown with respect to airspeed and altitude. This figure offers nearly all the required engine performance data except for part power conditions. In the majority of cases however, full throttle power is of the greatest interest to the user. Figure 61 shows the TPE331-10 PSFC and power lapse data produced by the MATLAB function when given the data from Figure 60 . 


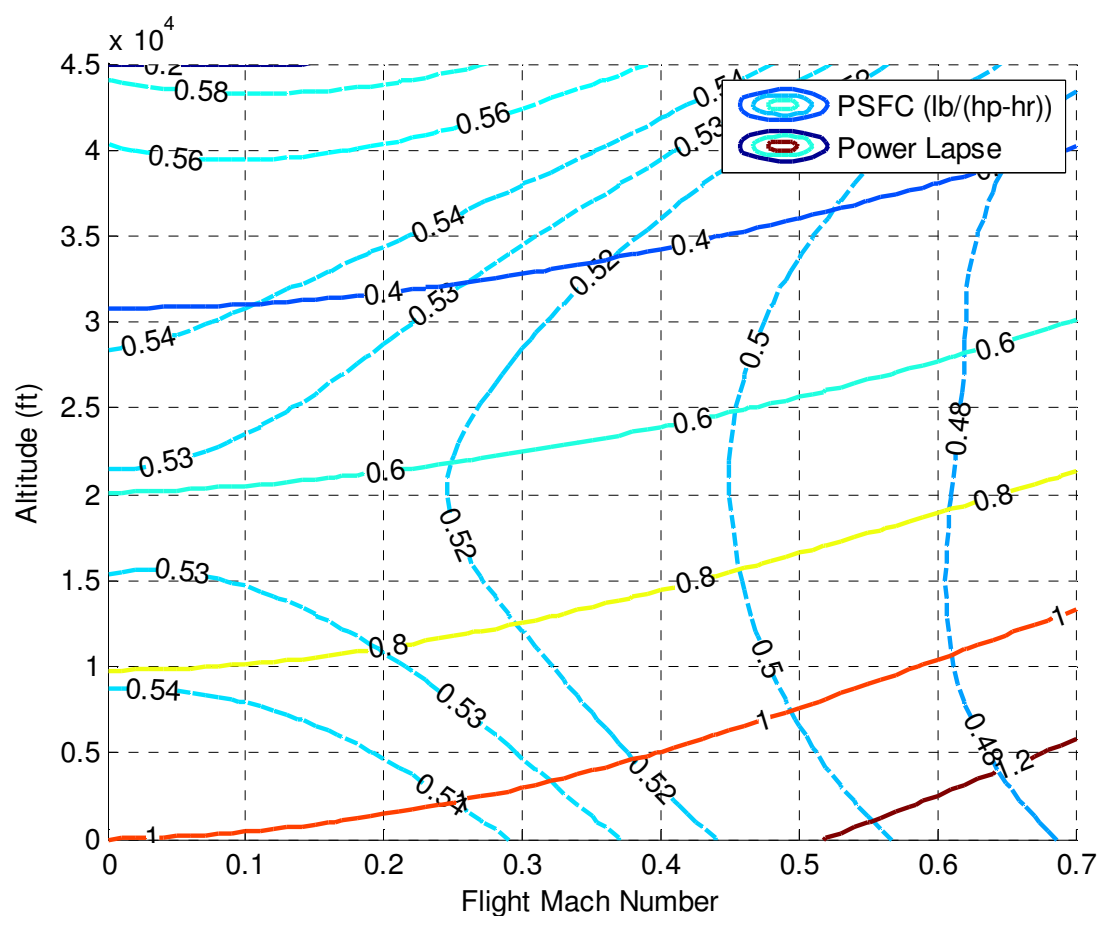

Figure 61. TPE331-10 PSFC and Power Lapse versus Mach Number at Full Power.

In this figure, the dashed lines show the engine's PSFC in $\mathrm{lb}_{\mathrm{m}} /(\mathrm{hp}-\mathrm{hr})$ while the solid lines shows the engine's power lapse. This engine's power lapse can exceed 1.0 due to an inlet ram effect at positive flight speeds.

The engine function must also have the ability to predict the power lapse and PSFC at part power if the user wishes to analyze the performance of the system at these part throttle conditions. In this TPE331-10 engine function, factors were added to predict the change in PSFC at part power. These factors were estimated using a computer engine deck for an Allison 250C20W turboshaft engine. This engine deck predicted an increase in PSFC of $2 \%$ at $80 \%$ power, and an increase of $97 \%$ at $20 \%$ power. Figure 62 shows the TPE331-10 PSFC and power lapse now versus altitude and power setting. 


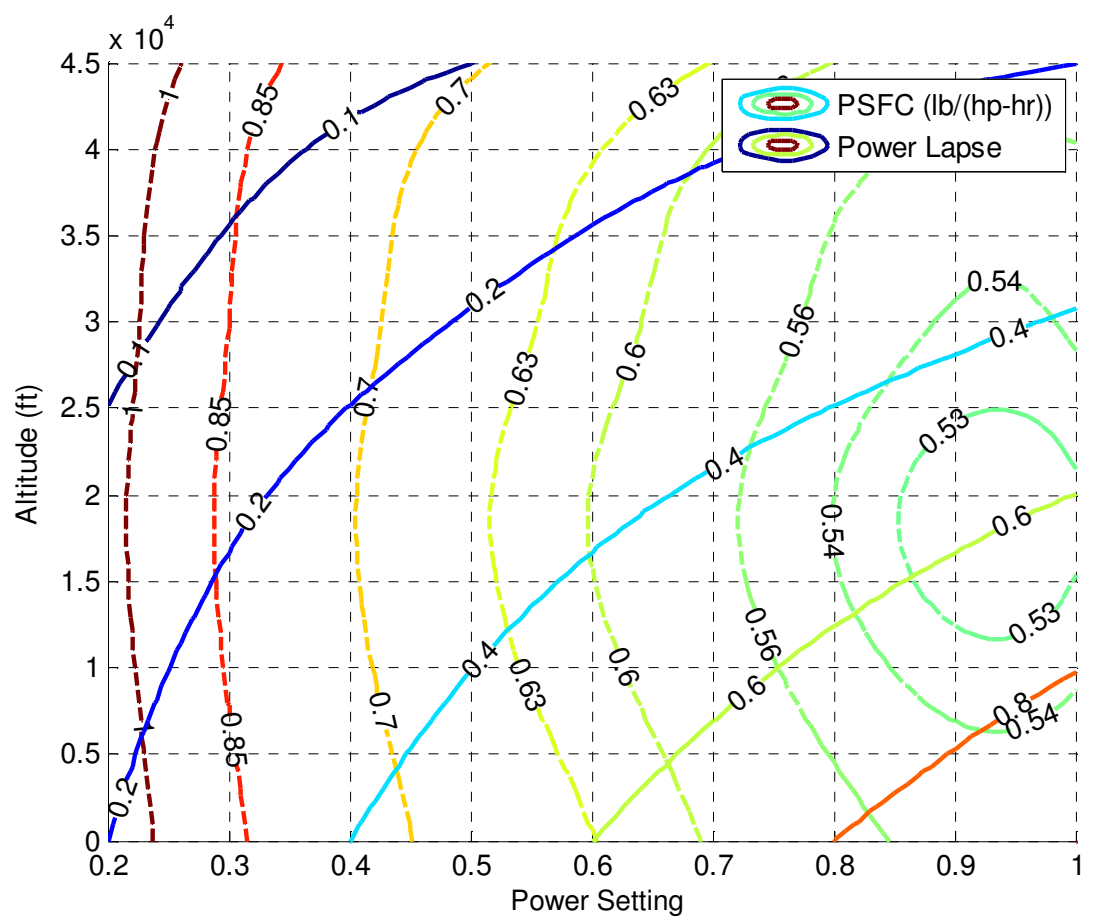

Figure 62. TPE331-10 PSFC and Power Lapse versus Power Setting at Zero Flight Speed.

In this figure, the dashed lines show the engine's PSFC while the solid lines shows the engine's power lapse. The minimum PSFC of this engine when modeled occurs at a power setting of approximately $92 \%$ at an altitude of $18,000 \mathrm{ft}$. This behavior is common for most internal combustion engines.

The behavior of PSFC with respect to engine size was also investigated. Generally speaking, the larger the turboshaft engine the lower its PSFC will be due to the increased efficiency of larger components. Figure 63 shows the sea level static PSFC at maximum power versus the maximum power for approximately 120 aircraft and 10 marine turboshaft engines. 


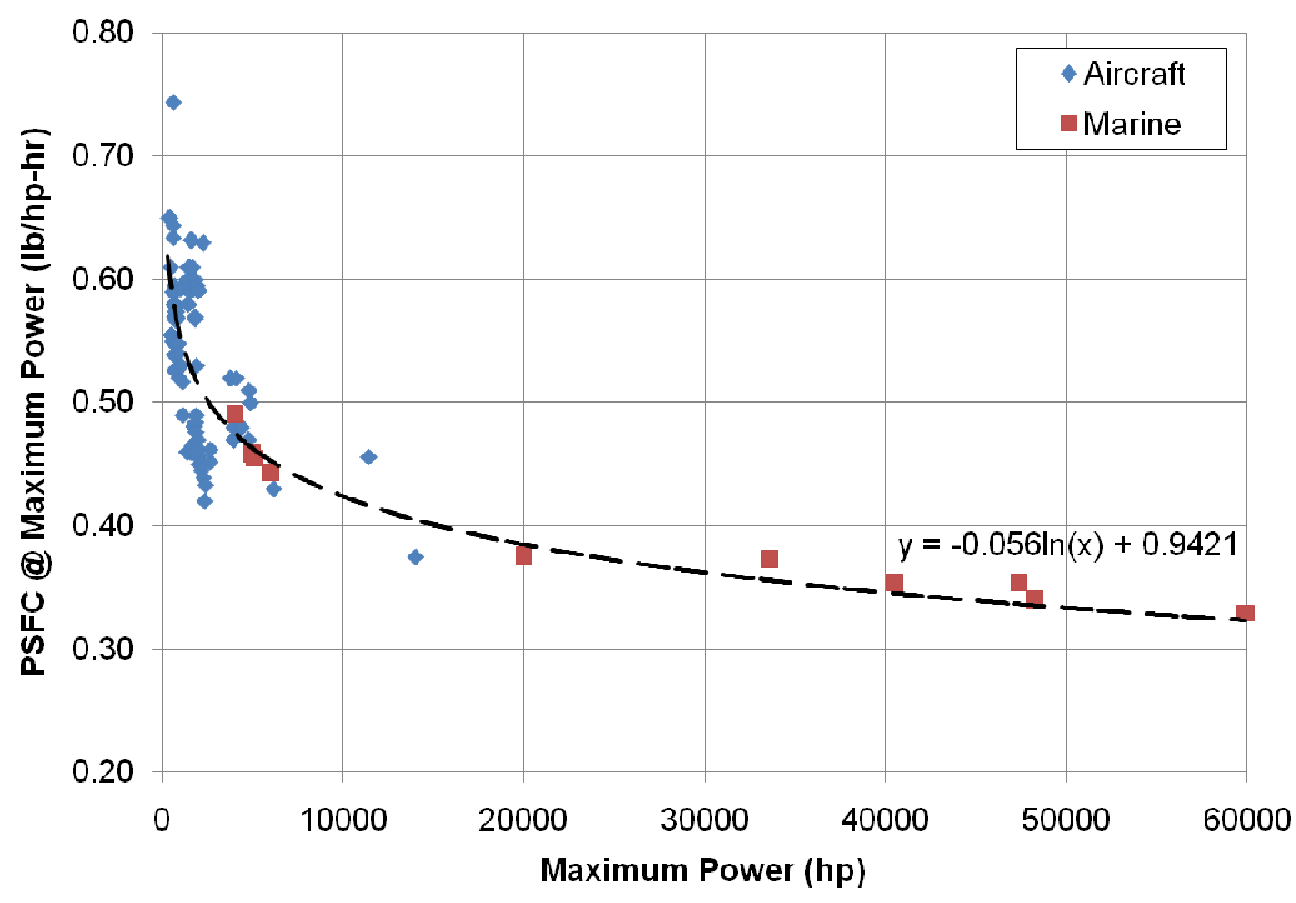

Figure 63. Turbine Engine SFC at Maximum Power versus Maximum Power.

In this figure the blue dots represent aircraft turboshaft engines while the red dots show marine turboshaft engines used primarily for power generation on commercial and naval ships. The aircraft engines range in power from $350 \mathrm{hp}$ to $14,000 \mathrm{hp}$, while the marine engines range from $6,000 \mathrm{hp}$ to $60,000 \mathrm{hp}$. It can be seen that the PSFC of these engines steadily improves with size and can be well-estimated with a logarithmic curve-fit. Until a detailed cycle analysis module is incorporated into HAPSS, this equation is utilized in the engine performance function to apply an adjustment on the PSFC based on the maximum output power of the engine.

\subsubsection{Performance}

The size of each engine is calculated during the on-design analysis. The engines must be large enough to supply hybrid propulsion system with enough electrical power to satisfy the power demanded by the fans while overcoming electrical losses. The output power of each engine is equal to the input power of each generator. However, the sea level static power of the engine is generally of most interest and is calculated as follows. 
where $P w r_{\text {eng }}$ is the output power of the engine in horsepower at the design point and $P L$ is the power lapse of the engine. The fuel flow to the engine in $\mathrm{lb}_{\mathrm{m}} / \mathrm{hr}$ is then calculated.

$$
\dot{w}_{f u e l}=P w r_{\text {eng }} P S F C
$$

The thermal efficiency of the engine is a useful parameter for comparisons to other propulsion system designs. The thermal efficiency is simply calculated by dividing the power outputted by the engine (shaft power) by the power inputted to the engine (fuel).

$$
\eta_{t h}=\frac{P w r_{e n g} L H V * 3600 * I}{\dot{w}_{f u e l} * J}
$$

where $L H V$ is the lower heating value of the fuel in $\mathrm{BTU} / \mathrm{lb} \mathrm{b}_{\mathrm{m}}$. The heat transfer rate of the engine is calculated in a similar fashion to that of the electric components.

$$
q_{\text {eng }}=\left(\frac{\dot{w}_{f u e l}}{3600 * L H V}\right)-\left(\frac{P w r_{\text {eng }} * I}{J}\right)
$$

Unlike the electric machines however, much of this heat is removed from the engine through the exhaust, alleviating the need for a robust engine cooling system. The ratio of heat removed in the exhaust to the heat transferred into the engine itself is unknown, although it should be assumed that the majority of heat is exhausted.

The mass flow rate of air through the engine is calculated next. This parameter is useful for determining the overall bypass ratio of the propulsion system. Unfortunately, without detailed cycle analysis the mass flow rate must be estimated using the user-inputted fuel-to-air ratio of the engine.

$$
\dot{m}_{\text {eng }}=\frac{\dot{w}_{\text {fuel }}}{3600} f
$$

where the mass flow rate is calculated in $\mathrm{lb}_{\mathrm{m}} / \mathrm{s}$ and the fuel flow rate is inputted in $\mathrm{lb}_{\mathrm{m}} / \mathrm{hr}$.

\subsubsection{Weight}

Similar to the electric motors and generators, the weight of the turbine engine is estimated using a curve-fit of modern turboshaft engines. The data set is the same used in Figure 63 except without the marine turbine engines. Marine turbines tend to be optimized for efficiency and reliability rather than weight. Figure 64 shows the empirical data for the aircraft turboshaft engines. 


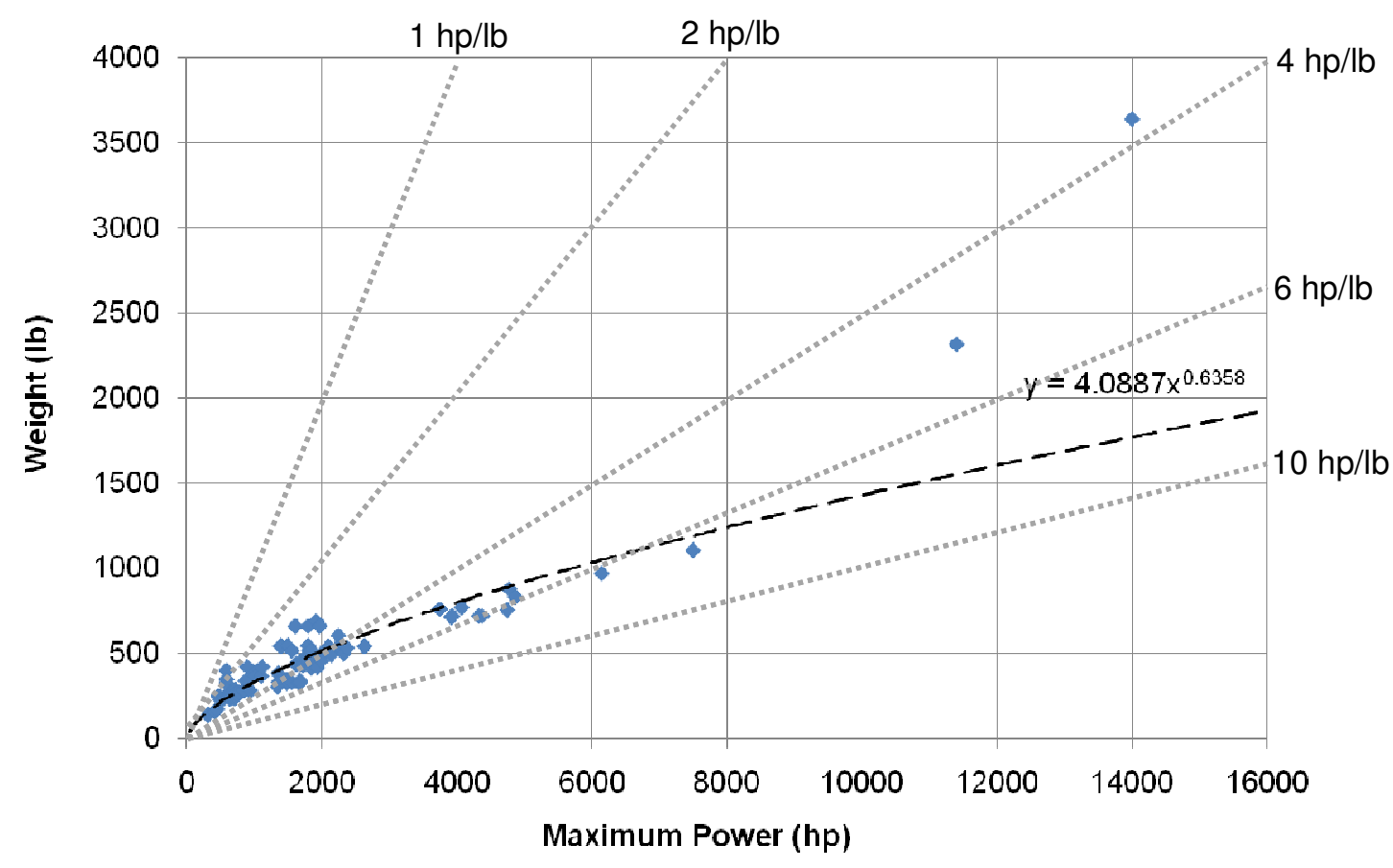

Figure 64. Aircraft Turboshaft Weight versus Maximum Power.

In this figure, the dotted gray lines represent constant power density while the dashed black line is the curve-fit equation. Most of the researched turboshaft engines have power densities between $2 \mathrm{hp} / \mathrm{lb}$ and $4 \mathrm{hp} / \mathrm{lb}$.

Future iterations of HAPSS could implement other weight estimation methods such as using a constant power density or implementing the NASA WATE methodology. The WATE method is currently used to estimate the weight of the fan (see section 4.6) and could be expanded to also estimate the turbine engine weight.

\subsection{Overall System Performance}

With the on-design analysis complete, HAPSS calculates some basic overall system performance metric which can be used for comparison with other propulsion system designs. The first metric is the propulsive efficiency.

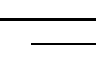

where $V_{6}$ is the velocity of the exhaust exiting the fan nozzle and $V_{a m b}$ is the flight speed of the aircraft, both in $\mathrm{ft} / \mathrm{s}$. This equation indicates that if the exhaust velocity equals the aircraft's velocity then the propulsive efficiency is $100 \%$. However, if the two velocities are the same then 
no thrust is being produced. The principal conclusion from this is that it is more efficient to slightly accelerate a large amount of air than greatly accelerate a small amount of air.

Next, the thermal efficiency of the system is calculated. The thermal efficiency is the ratio of propulsive power to fuel power; or in other words the efficiency of converting the fuel's chemical energy into useful kinetic energy for moving the aircraft.

$$
\eta_{t h}=\frac{\left(\frac{m_{f} n_{f a n} V_{b}^{2}}{2}\right)-\left(\frac{m_{f} n_{f a n} V_{a m b}^{2}}{2}\right)}{\frac{\dot{w}_{f u e l} n_{\text {eng }}}{3600} L H V * J * g_{c}}
$$

The thermal efficiency is useful for comparing engine and propulsion system architectures such at hybrid systems, turbofans, turbojets, turboprops, unducted fans, etc. The hybrid system will likely have a lower thermal efficiency due to the electrical inefficiencies that exist between the power producer and the thrust producer. The overall efficiency is simply the product of the propulsive and thermal efficiencies.

$$
\eta_{o}=\eta_{p} \eta_{t h}
$$

Another performance metric that is useful for comparison with traditional propulsion systems is the bypass ratio. In a turbo fan engine, the bypass ratio is ratio of air that passes through the bypass (secondary flow) duct to the air passing through the engine core (primary flow). The bypass ratio of hybrid propulsion system however is the ratio of the air passing through the fans to the air passing through the engines, as shown here.

$$
B P R=\frac{n_{f a n} \dot{m}_{f}}{\dot{m}_{\text {eng }}}
$$

The final overall system performance metric that is calculated is the thrust specific fuel consumption (TSFC) in $\mathrm{lb}_{\mathrm{m}} /\left(\mathrm{lb}_{\mathrm{f}}-\mathrm{hr}\right)$.

$$
T S F C=\frac{\dot{w}_{\text {fuel }} n_{\text {eng }}}{F n_{\text {fan }}}
$$

where $F$ is the thrust of a single fan in $\mathrm{lb}_{\mathrm{f}}$. TSFC is one of the most useful parameters for comparing aircraft efficiency with respect to fuel consumption. 


\section{Off-Design Methodology}

With the on-design analysis complete, the hybrid propulsion system has been sized to meet the demands of the design condition. The next step is to look at an off-design condition to determine how the system performs. Figure 65 shows the HAPSS flow diagram with the upper on-design portion blurred. Section 5 will focus on the lower off-design portion.

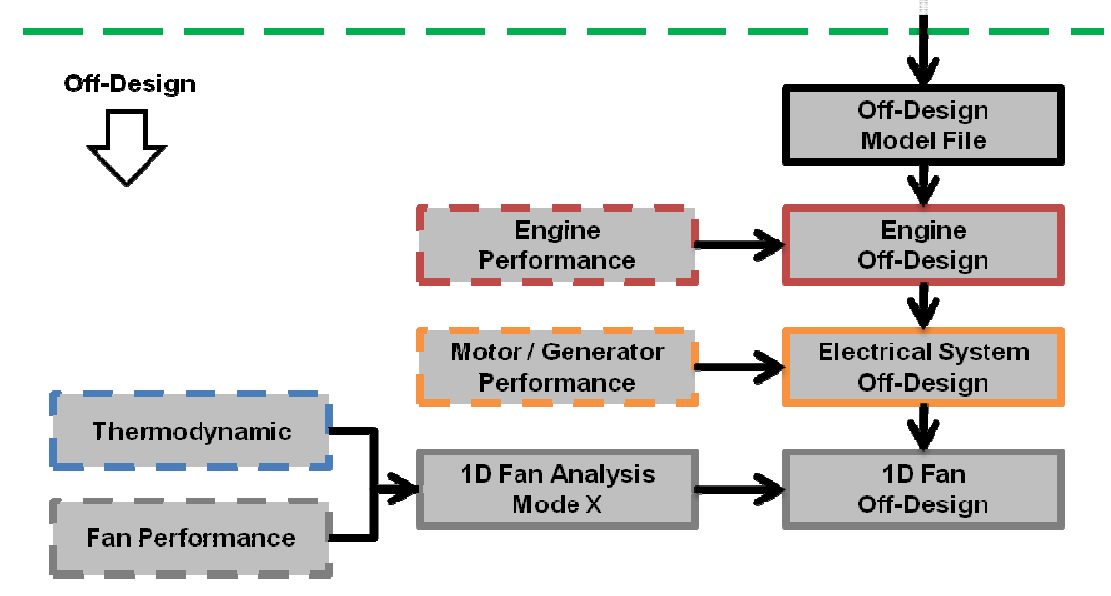

Figure 65. Detailed Off-Design Logic Flow Diagram of HAPSS. 
The off-design inputs for the electrical system and engine are located in this module as well. The user may set these inputs to equal those from the on-design condition which is useful since many parameters will not change.

\subsection{Engine Analysis}

The engine analysis begins by using the engine performance function to determine the power lapse and PSFC of the engine at the off-design condition. This script also checks the power lapse and PSFC values given by the engine performance function to determine they are real values (the function will output "NaN" if the inputted flight condition is out of bounds of the data). The output power of the engine at the off-design is calculated as follows.

$$
P w r_{\text {eng }}=P w r_{\text {eng, } \max } P L
$$

where $P L$ is the power lapse at the off-design condition and $P w r_{e n g, m a x}$ is the maximum output power of the engine in horsepower, calculated during the on-design analysis.

This script then continues to calculate the same performance data that was computed during the on-design condition such as fuel flow rate, thermal efficiency, and heat transfer rate.

\subsection{Electrical System Analysis}

The computations for the electrical system are performed in a similar manner as the ondesign case, but in reverse. Consider the off-design case in which the battery is discharging, modeled by Figure 66 from section 4.8.3.

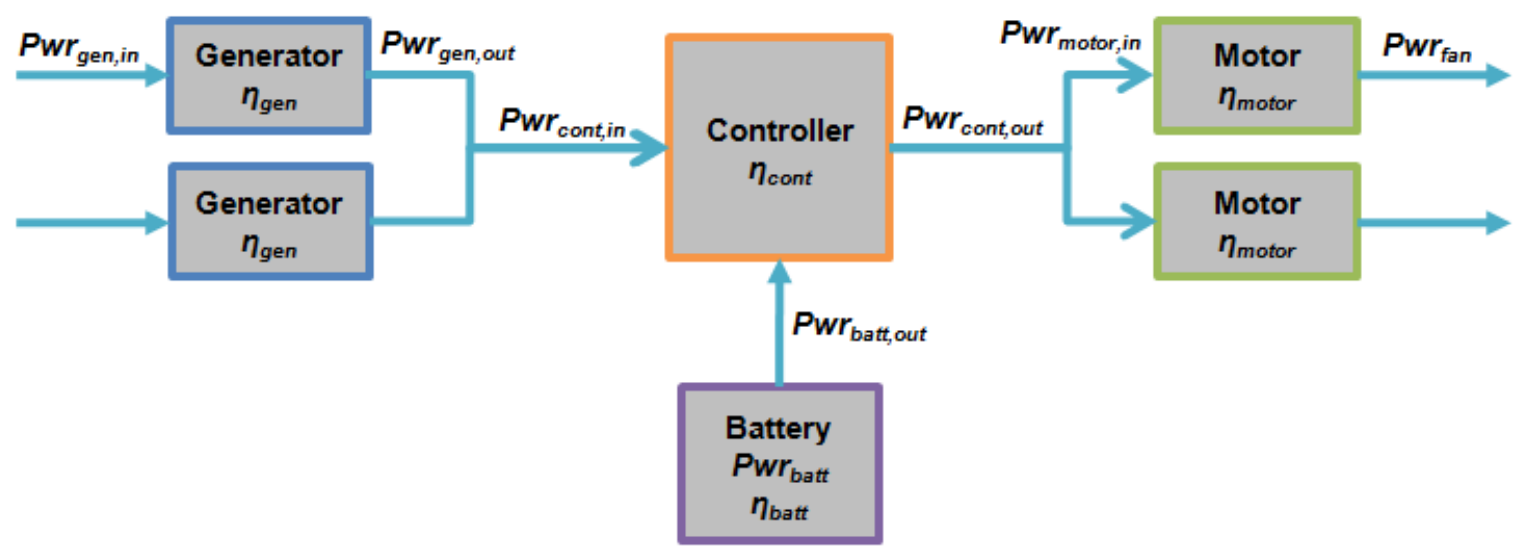

Figure 66. Electrical System Diagram, Battery Discharging. 
The known variables in this diagram in the on-design case were the power supplied to the fan, the power supplied by the battery, and the efficiency of each component. In the off-design case however the power supplied to the fan is unknown and must be solved for. Instead, the power provided to the generator in known based on the engine performance. The battery discharging (or charging) power and the generator, controller, and battery efficiencies are also known.

The generator efficiency is calculated using the generator's efficiency map similar to the on-design case (if the efficiency is provided as a function instead of a scalar). With the size of the generator fixed at the on-design condition, the speed and torque of the generator can be used to calculate its efficiency. As with the on-design case, the off-design generator speed is inputted by the user. The controller and battery efficiencies will generally be the same as the on-design case, although HAPSS accepts other values for the off-design analysis.

The off-design motor efficiency however requires an iterative approach if it is too supplied as a function and not a scalar. The efficiency of the motor is dependent on its speed. The speed depends on the output power of the motor (the speed is calculated during the 1D fan analysis). And the output power depends on the efficiency. Therefore, an initial guess for the motor's efficiency is used and the fan 1D performance is executed to determine the fan/motor speed. This speed is then used to calculate a new motor efficiency and this process is repeated until the error is below the user-specified tolerance.

The electrical system off-design analysis then proceeds to calculate the overall system efficiency and the heat transfer rate of each component similar to the on-design case.

\subsection{D Fan Analysis}

With the motor output power calculated, the off-design analysis for the $1 \mathrm{D}$ fan performance is performed next. This script is responsible for checking the inputs to the $1 \mathrm{D}$ fan analysis and calling the correct mode. The modes described in section 4.5 may also be used in the off-design case. In the off-design case however, two of the six main design variables to the 1D fan analysis will typically be the fan diameter and power. The user can then select the mode based on the 
third design variable he or she has available; fan pressure ratio, fan face Mach number, mass flow rate, or thrust.

As described in section 4.5.12 however, mode 12 is capable of computing the 1D fan performance by only knowing the diameter and power. This is possible using fan affinity laws calculated during the on-design analysis. These laws can predict off-design fan performance with small changes in speed and efficiency. 


\section{Aircraft Example}

In this section an example aircraft with a hybrid propulsion system is analyzed using HAPSS. The exact size and configuration of the vehicle is arbitrary with respect to the propulsion system analysis. However, the size and performance of the propulsion system is comparable to that of a 150 passenger regional airliner. This vehicle will utilize a total of 20 propulsive fans and two turbine-powered electric generators.

The purpose of this section is not to present a propulsion system design for an aircraft, but rather to demonstrate the capabilities of the HAPSS code.

\subsection{On-Design Analysis}

The design point of the aircraft, which is the flight condition to which the aircraft will be sized, is top-of-climb at an altitude of 35,000 feet and a speed of Mach 0.7. At this point, it will be assumed that the aircraft requires $10,000 \mathrm{lb}_{\mathrm{f}}$ of total net thrust at maximum power. HAPSS will begin by sizing the propulsive fans such that they satisfy this thrust requirement at the flight condition. Then the program will size the electrical system including the motors, generators, and controller. Finally, the turbine engines will be sized and overall performance of the propulsion system can be calculated.

At the design point, a number of trades will also be performed to investigate the affect some of the design variables have on the size and performance of the propulsion system. These trades are representative of those an engineer would wish to consider during the design stage of a hybrid propulsion system.

\subsubsection{D Fan Analysis}

Before the 1D fan performance analysis can be performed, some simple assumptions and inputs had to be defined. The inputs to the 1D fan performance analysis for this example are listed here in Table 8. These inputs are defined per fan. 
Table 8. On-Design 1D Fan Performance Inputs.

\begin{tabular}{|l|c|c|}
\hline \multicolumn{1}{|c|}{ Input } & Units & Value \\
\hline Altitude & $\mathrm{ft}$ & 35,000 \\
\hline Flight Mach number & - & 0.7 \\
\hline Throttle setting & $\%$ & $100 \%$ \\
\hline 1D fan analysis mode & - & 4 \\
\hline Thrust required & $\mathrm{Ib}_{\mathrm{f}}$ & 500 \\
\hline Fan face Mach number & - & 0.6 \\
\hline Number of fans & - & 20 \\
\hline Fan polytropic efficiency & $\%$ & 95 \\
\hline Inlet pressure recovery function & - & @PR2parabolic \\
\hline Nozzle pressure drop & $\%$ & 1 \\
\hline Nozzle velocity coefficient & $\%$ & 99 \\
\hline Hub-to-tip ratio & - & 0.3 \\
\hline Fan tip Mach number & - & 1.0 \\
\hline Fuel-to-air ratio & - & 0.0 \\
\hline
\end{tabular}

The 1D fan performance mode in this example will optimize the fan pressure ratio to minimize the power required by the fan at the design point. The inlet pressure recovery will be defined by a parabolic function versus flight Mach number; this function was shown in Figure 17. It was assumed that each fan will be sized equally and must produce the same amount of thrust. The outputs from the 1D fan performance analysis are shown here in Table 9, defined per fan. 
Table 9. On-Design 1D Fan Performance Outputs.

\begin{tabular}{|l|c|c|}
\hline \multicolumn{1}{|c|}{ Input } & Units & Value \\
\hline Fan diameter & $\mathrm{ft}$ & 2.62 \\
\hline Hub diameter & $\mathrm{ft}$ & 0.79 \\
\hline Fan face area & $\mathrm{ft}^{2}$ & 4.92 \\
\hline Fan exit area & $\mathrm{ft}^{2}$ & 4.04 \\
\hline Fan pressure ratio & - & 1.30 \\
\hline Mass flow rate & $\mathrm{lb}_{\mathrm{m}} / \mathrm{s}$ & 71.9 \\
\hline Corrected mass flow rate & $\mathrm{lb}_{\mathrm{m}} / \mathrm{s}$ & 205 \\
\hline Volumetric flow rate & $\mathrm{ft}^{3} / \mathrm{s}$ & 280 \\
\hline Corrected thrust & $\mathrm{lb}$ & 1562 \\
\hline Power required & $\mathrm{hp}$ & 877 \\
\hline Fan torque & $\mathrm{ft}-\mathrm{lb}$ & 643 \\
\hline Inlet pressure recovery & $\%$ & 98 \\
\hline Fan rotational speed & $\mathrm{RPM}$ & 7168 \\
\hline Fan blade tip velocity & $\mathrm{ft} / \mathrm{s}$ & 985 \\
\hline Nozzle pressure ratio & - & 1.76 \\
\hline Nozzle exit velocity & $\mathrm{ft} / \mathrm{s}$ & 905 \\
\hline Enthalpy rise across fan & $\mathrm{BTU} / \mathrm{b}_{\mathrm{m}}$ & 8.63 \\
\hline Adiabatic efficiency & $\%$ & 94.8 \\
\hline Power coefficient & - & 0.0701 \\
\hline Flow coefficient & - & 0.0216 \\
\hline Propulsive efficiency & $\%$ & 85.9 \\
\hline
\end{tabular}

The 1D fan analysis also outputs various code-related parameters such as tolerances, errors and number of iterations (for iterative solutions), as well as all of the inputs supplied by the user. In this example, the 1D fan analysis determined that a fan pressure ratio of 1.30 resulted in the minimum power required by the fan. However, the fan pressure ratio impacts many other aspects of the fan's performance, and the designer would likely be interested in such effects.

Figure 67 through Figure 72 show trades of various fan performance metrics versus fan pressure ratio and inlet pressure recovery, the other input parameters remained the same as those listed in Table 8. These trades include inlet pressure recovery to show its significant impact on the performance of the fan. For example, an inlet pressure recovery of 0.96 would have yielded an optimal fan pressure ratio of 1.42 as seen in Figure 67. Each colored line represents a constant inlet pressure recovery as labeled in Figure 67, and these lines represent the same inlet pressure recovery in the subsequent figures. The red dot in each figure represents the aircraft from this example. 
The following figures were produced by HAPSS by analyzing a wide variety of on-design points. The user can quickly create trades like these by executing the HAPSS code inside forloops where certain parameters (in this case fan pressure ratio and inlet pressure recovery) are varied.

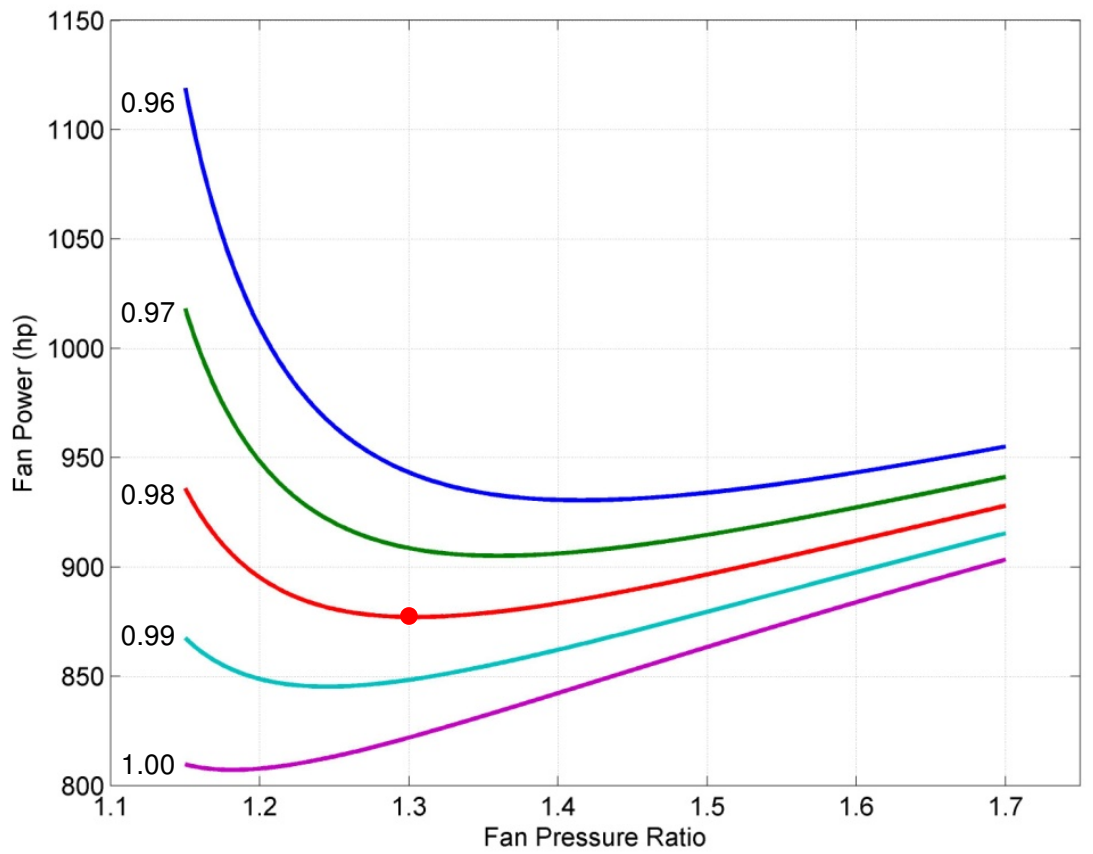

Figure 67. Fan Power versus Fan Pressure Ratio and Inlet Pressure Recovery. 


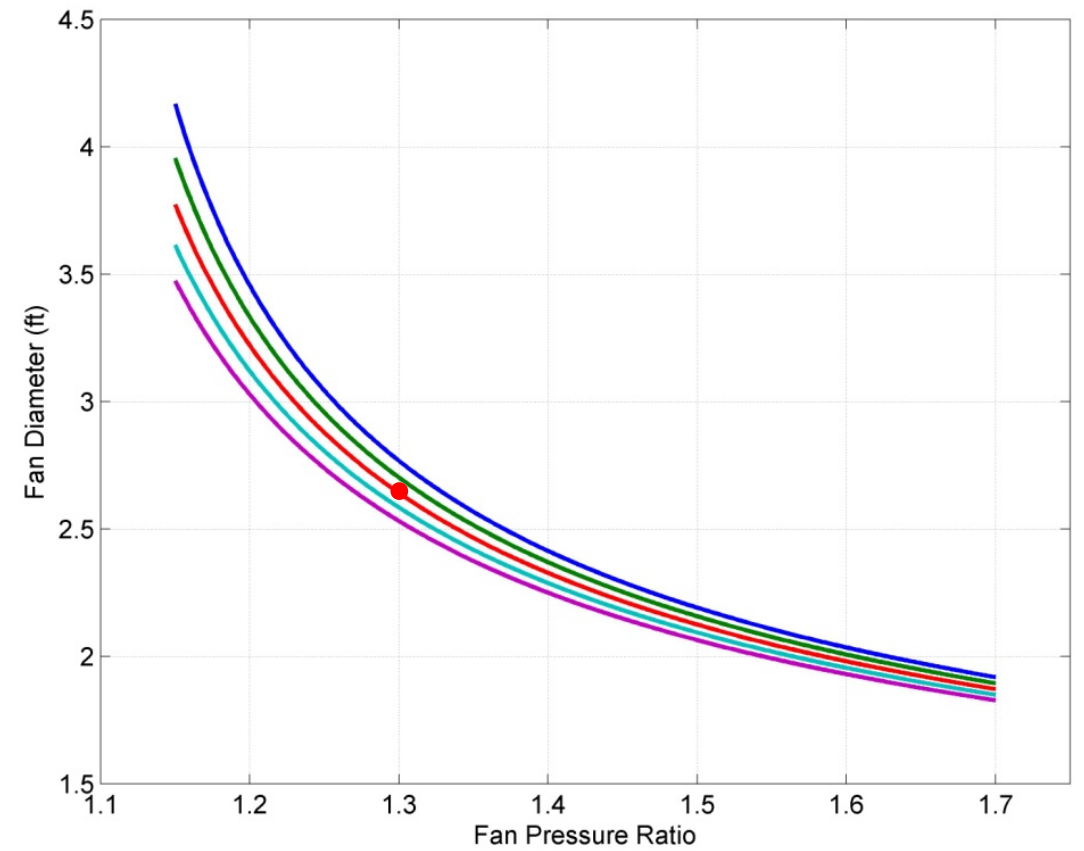

Figure 68. Fan Diameter versus Fan Pressure Ratio and Inlet Pressure Recovery.

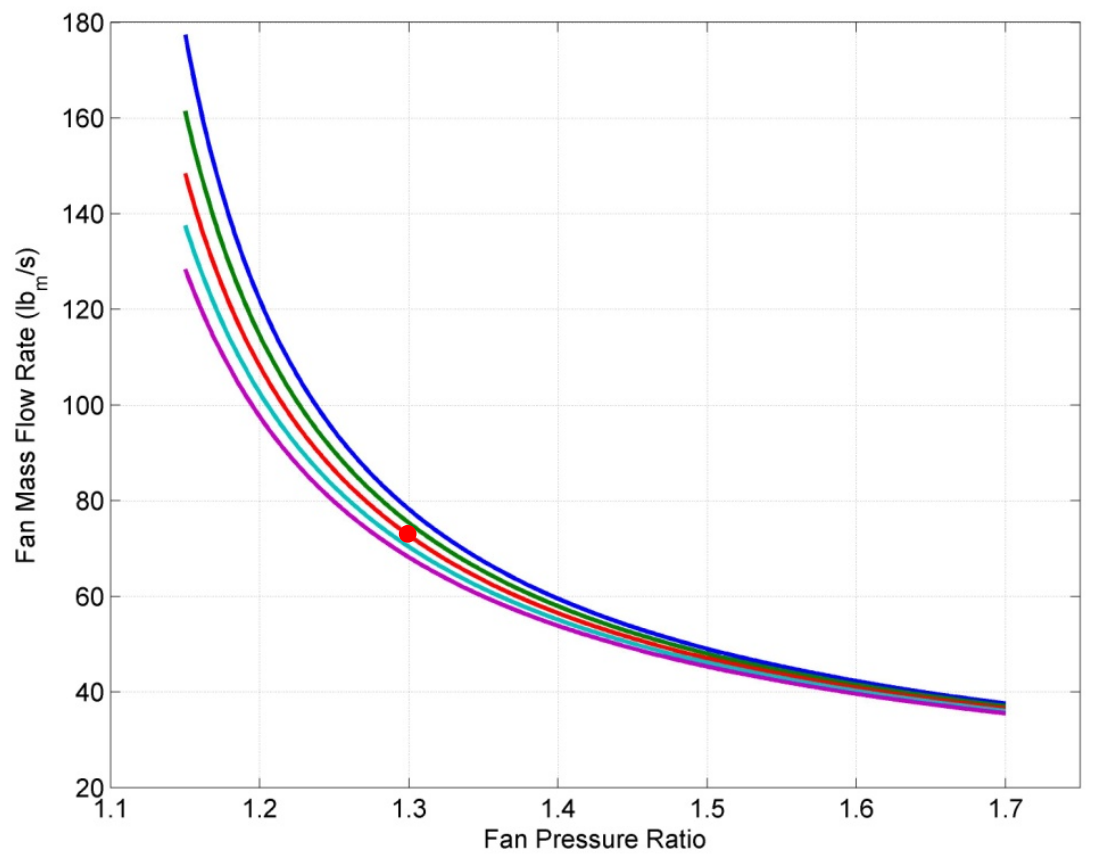

Figure 69. Fan Mass Flow Rate versus Fan Pressure Ratio and Inlet Pressure Recovery. 


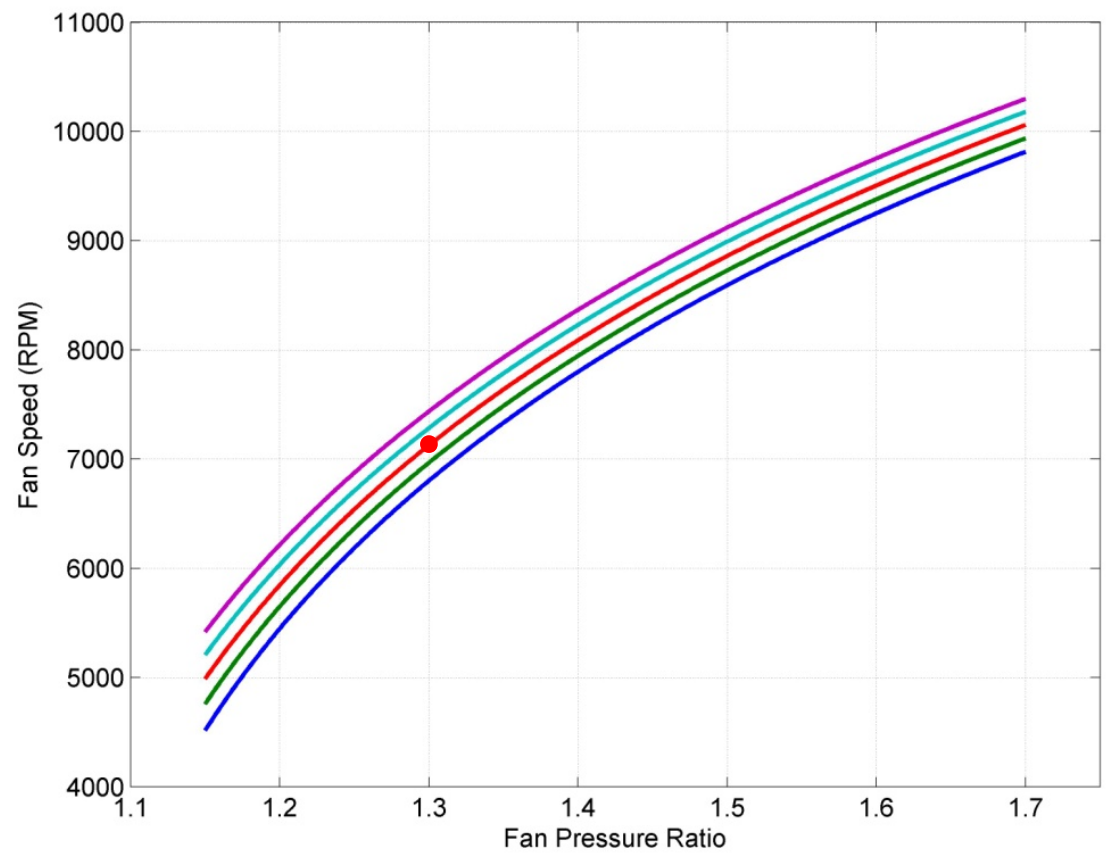

Figure 70. Fan Speed versus Fan Pressure Ratio and Inlet Pressure Recovery.

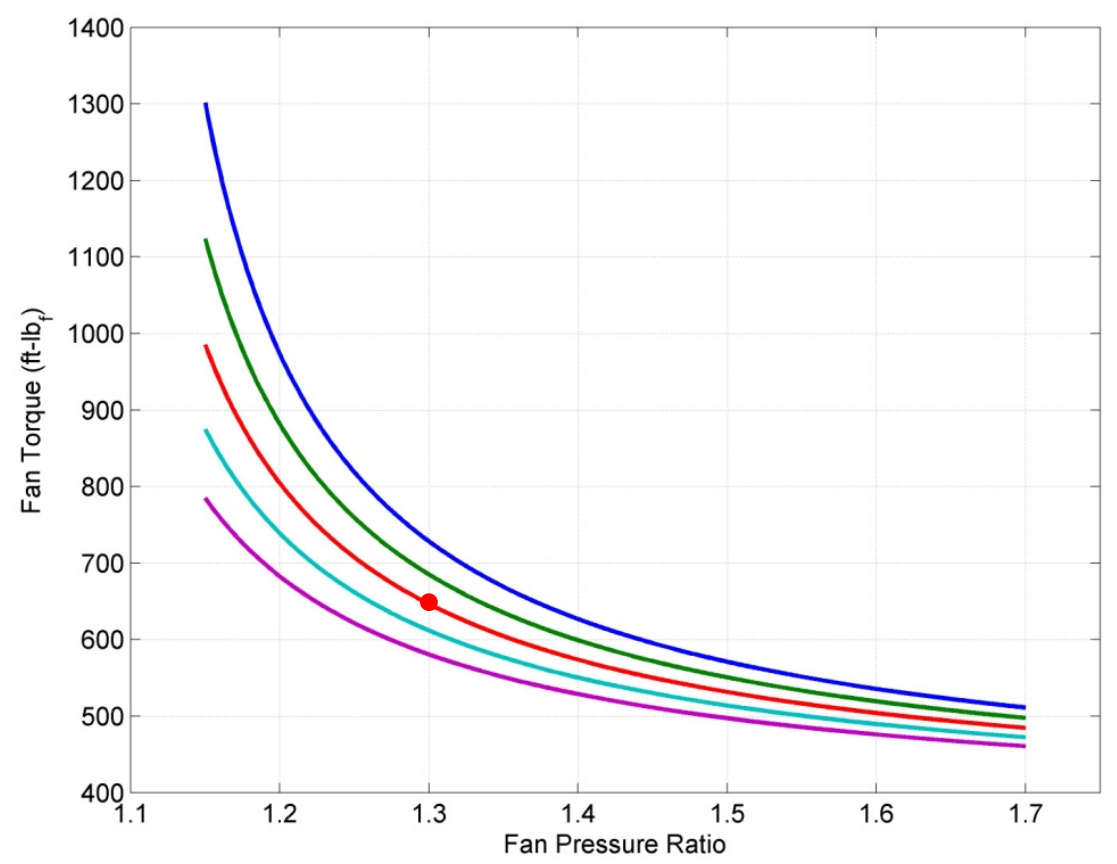

Figure 71. Fan Torque versus Fan Pressure Ratio and Inlet Pressure Recovery. 


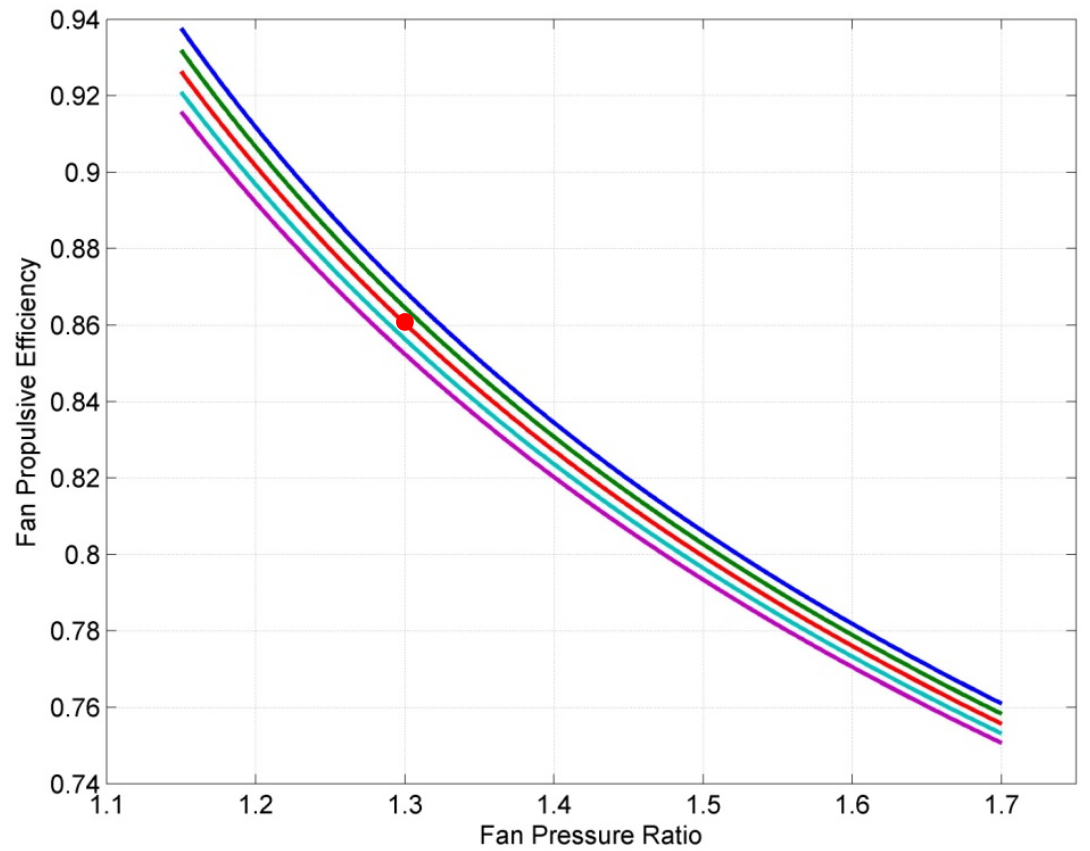

Figure 72. Fan Propulsive Efficiency versus Fan Pressure Ratio and Inlet Pressure Recovery.

These figures reveal that increasing the fan pressure ratio will lead to a smaller fan propulsor with less mass flow rate, but at an increased speed and reduced propulsive efficiency. Figure 67 is of greatest interest because it illustrates that the power required by the fan reaches a minimum value at some optimal fan pressure ratio (mode 4 of the fan $1 \mathrm{D}$ performance finds this minimum optimal fan pressure ratio). This is important because except for the fans, the weight of the components in the hybrid propulsion system is determined by power in this version of HAPSS. However, there are other aspects of the propulsion system which must be considered when choosing a design point fan pressure ratio.

At the design point the user may also be interested in the effect of the number of fans on the propulsion system performance. The following on-design trades were performed by varying the number of fans from six to 40 with lines of constant fan pressure ratio. For these trades the inlet pressure recovery was kept constant at 0.98 which was the output from the $1 \mathrm{D}$ fan on-design analysis. 
Figure 73 through Figure 76 show the results of these trades with the colored lines representing constant fan pressure ratio as labeled in Figure 73. As before, these figures were created by running a wide variety of on-design points with HAPSS.

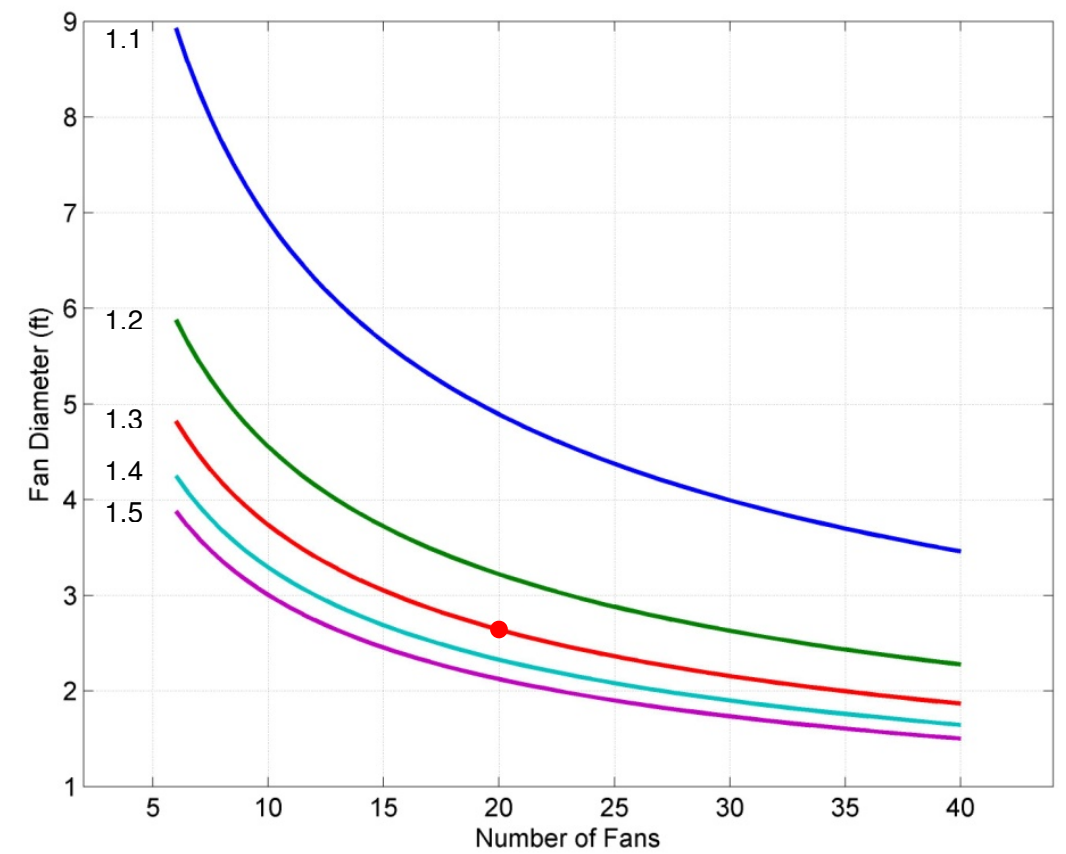

Figure 73. Fan Diameter versus Number of Fans and Fan Pressure Ratio. 


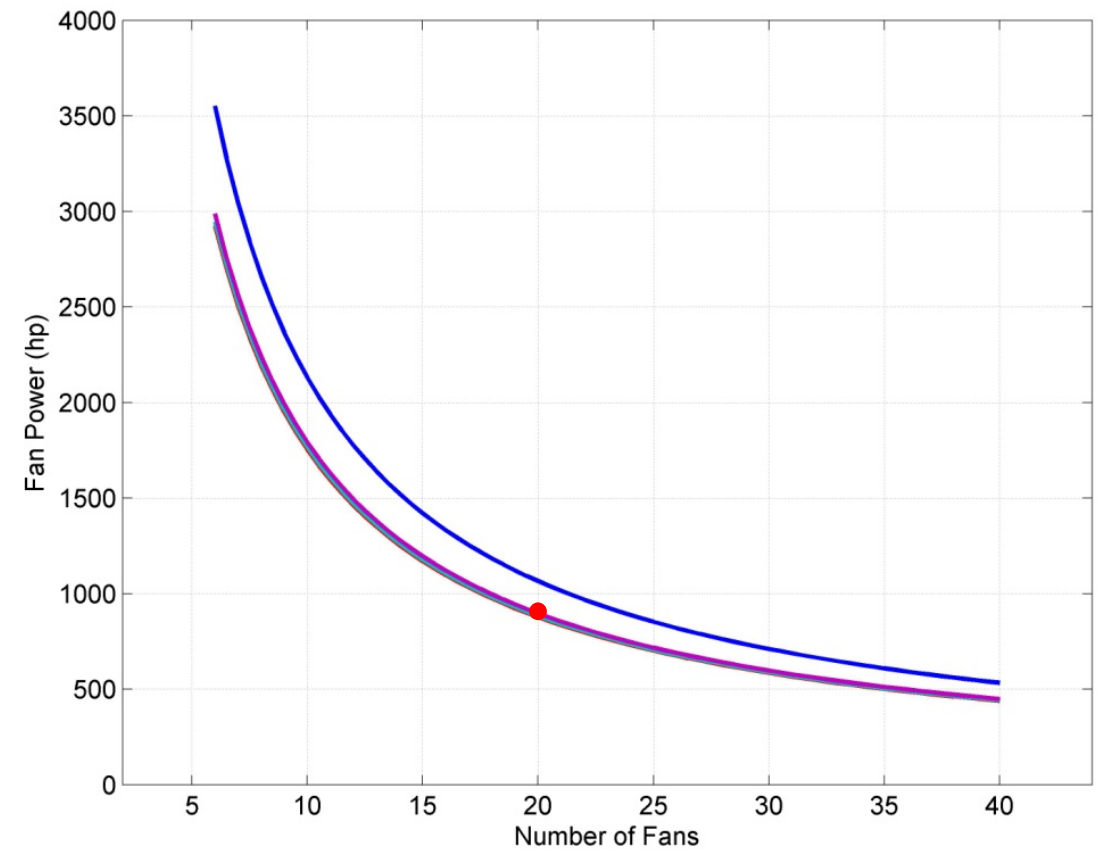

Figure 74. Fan Power versus Number of Fans and Fan Pressure Ratio.

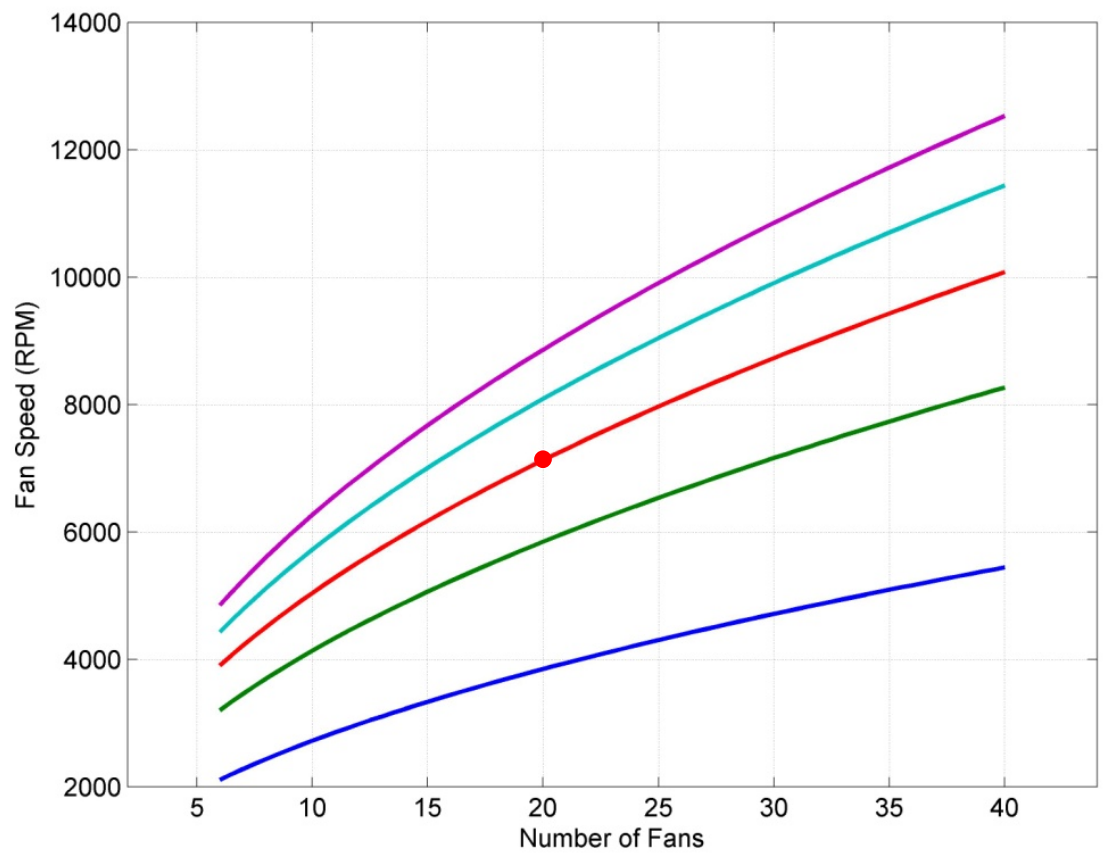

Figure 75. Fan Speed versus Number of Fans and Fan Pressure Ratio. 


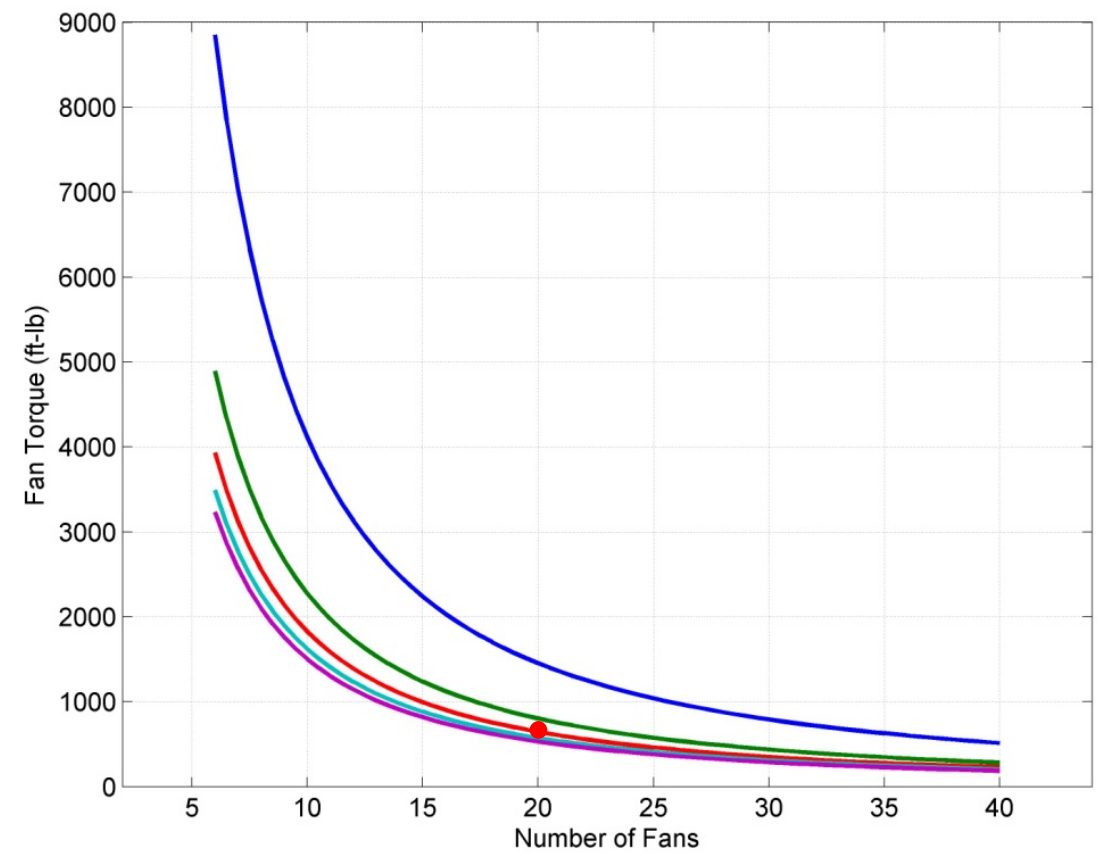

Figure 76. Fan Torque versus Number of Fans and Fan Pressure Ratio.

These figures reveal that increasing the number of fans will yield smaller fans due to the reduced thrust each one must produce. This also corresponds to a reduction in individual fan power and torque. At higher fan pressure ratios the number of fans appears to have a minimal impact on the total fan weight. Changing the number of fans also has a significant structural and aerodynamic impact on the aircraft, effects which are not represented here.

Fan pressure ratio, number of fans, and inlet pressure recovery are just a few of the numerous variables that the user has available to trade during the 1D fan analysis.

\subsubsection{Fan Weight Analysis}

With the on-design 1D fan analysis complete the fan weight is determined next. Table 10 lists the inputs to the fan weight analysis that was used in this example. 
Table 10. On-Design Fan Weight Inputs.

\begin{tabular}{|l|c|c|}
\hline \multicolumn{1}{|c|}{ Input } & Units & Value \\
\hline Rotor blade material density & $\mathrm{lb}_{\mathrm{m}} / \mathrm{ft}^{3}$ & 281 \\
\hline Stator blade material density & $\mathrm{lb}_{\mathrm{m}} / \mathrm{ft}^{3}$ & 281 \\
\hline Rotor disc material density & $\mathrm{lb}_{\mathrm{m}} / \mathrm{ft}^{3}$ & 281 \\
\hline Hardware material density & $\mathrm{lb}_{\mathrm{m}} / \mathrm{ft}^{3}$ & 281 \\
\hline Case material density & $\mathrm{Ib}_{\mathrm{m}} / \mathrm{ft}^{3}$ & 281 \\
\hline Shaft material density & $\mathrm{lb}_{\mathrm{m}} / \mathrm{ft}^{3}$ & 281 \\
\hline Shaft material yield stress & $\mathrm{lb}_{\mathrm{f}} / \mathrm{ft}^{2}$ & $19,296,000$ \\
\hline Case thickness & $\mathrm{ft}$ & 0.0104 \\
\hline Blade volume factor & - & 0.055 \\
\hline Rotor blade aspect ratio & - & 2.0 \\
\hline Stator blade aspect ratio & - & 3.0 \\
\hline Rotor blade taper ratio & - & 1.0 \\
\hline Rotor solidity & - & 0.9 \\
\hline Stator solidity & - & 1.1 \\
\hline Rotor overspeed factor & - & 1.05 \\
\hline Shaft factor of safety & - & 2.0 \\
\hline Number of frames & - & 1 \\
\hline Frame type & - & 1 \\
\hline
\end{tabular}

The fan weight analysis uses the density of titanium for all of the components with a case thickness of $1 / 8 \mathrm{in}$. The blade aspect ratio, taper ratio, and solidity are representative of modern turbofan fan rotor and stator blades. The frame type is a single-bearing without power takeoff. The outputs of the fan weight analysis are shown in Table 11, defined per fan. 
Table 11. On-Design Fan Weight Outputs.

\begin{tabular}{|l|c|c|}
\hline \multicolumn{1}{|c|}{ Output } & Units & Value \\
\hline Shaft diameter & $\mathrm{ft}$ & 0.07 \\
\hline Number of rotor blades & - & 17 \\
\hline Number of stator blades & - & 30 \\
\hline Maximum blade tip speed & $\mathrm{ft} / \mathrm{s}$ & 1035 \\
\hline Disc volume & $\mathrm{ft}^{3}$ & 0.054 \\
\hline Rotor blade volume & $\mathrm{ft}^{3}$ & 0.0096 \\
\hline Stator blade volume & $\mathrm{ft}^{3}$ & 0.0042 \\
\hline Disc stress & $\mathrm{lb}_{\mathrm{f}} / \mathrm{ft}^{2}$ & 4254400 \\
\hline Relative disc stress & $\mathrm{l} \mathrm{b}_{\mathrm{f}} / \mathrm{ft}$ & 16.1 \\
\hline Relative disc thickness & $\mathrm{ft}$ & 0.094 \\
\hline Rotor length & $\mathrm{ft}$ & 0.44 \\
\hline Stator length & $\mathrm{ft}$ & 0.30 \\
\hline Rotor blade chord & $\mathrm{ft}$ & 0.44 \\
\hline Stator blade chord & $\mathrm{ft}$ & 0.30 \\
\hline Case weight & $\mathrm{lb}_{\mathrm{m}}$ & 18.9 \\
\hline Disc weight & $\mathrm{lb}_{\mathrm{m}}$ & 15.2 \\
\hline Frame weight & $\mathrm{lb}_{\mathrm{m}}$ & 71.5 \\
\hline Hardware weight & $\mathrm{lb}_{\mathrm{m}}$ & 2.6 \\
\hline Rotor weight & $\mathrm{lb}_{\mathrm{m}}$ & 45.6 \\
\hline Stator weight & $\mathrm{lb}_{\mathrm{m}}$ & 35.8 \\
\hline Total fan assembly weight & $\mathrm{lb}_{\mathrm{m}}$ & 190.3 \\
\hline
\end{tabular}

The fan weight analysis indicates that the each fan assembly for this vehicle weighs 190.3 lbs. It can be seen that the frame weight contributes significantly to the total assembly weight. The analysis for the frame weight may be an overestimation for a distributed propulsion system with many small fans. It is believed that the weight formulation is better suited for systems with just two to four large turbofan engines.

As with the 1D performance analysis, the designer may be interested in how the fan pressure ratio, inlet pressure recovery, and the number of fans affect the weight of the fans. Figure 77 shows the effect of fan pressure ratio and inlet pressure recovery on the fan weight, while Figure 78 shows how fan weight changes with the number of fans. 


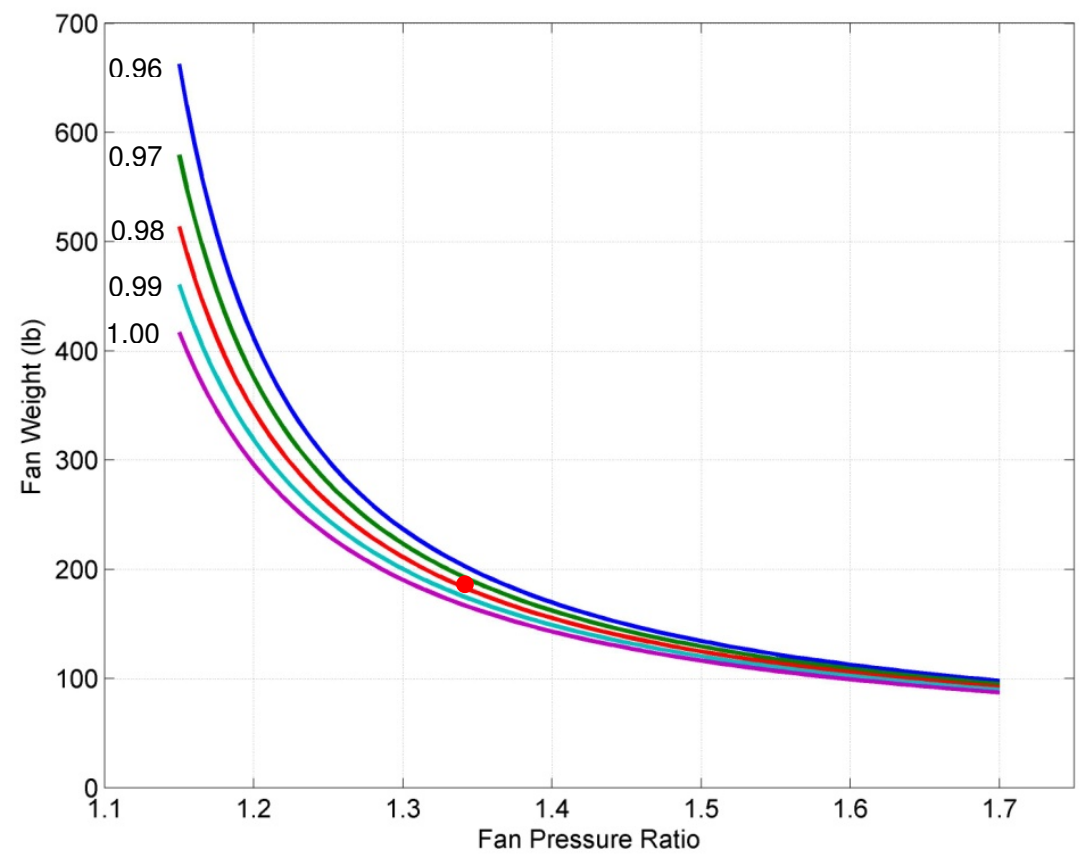

Figure 77. Fan Weight versus Fan Pressure Ratio and Inlet Pressure Recovery.

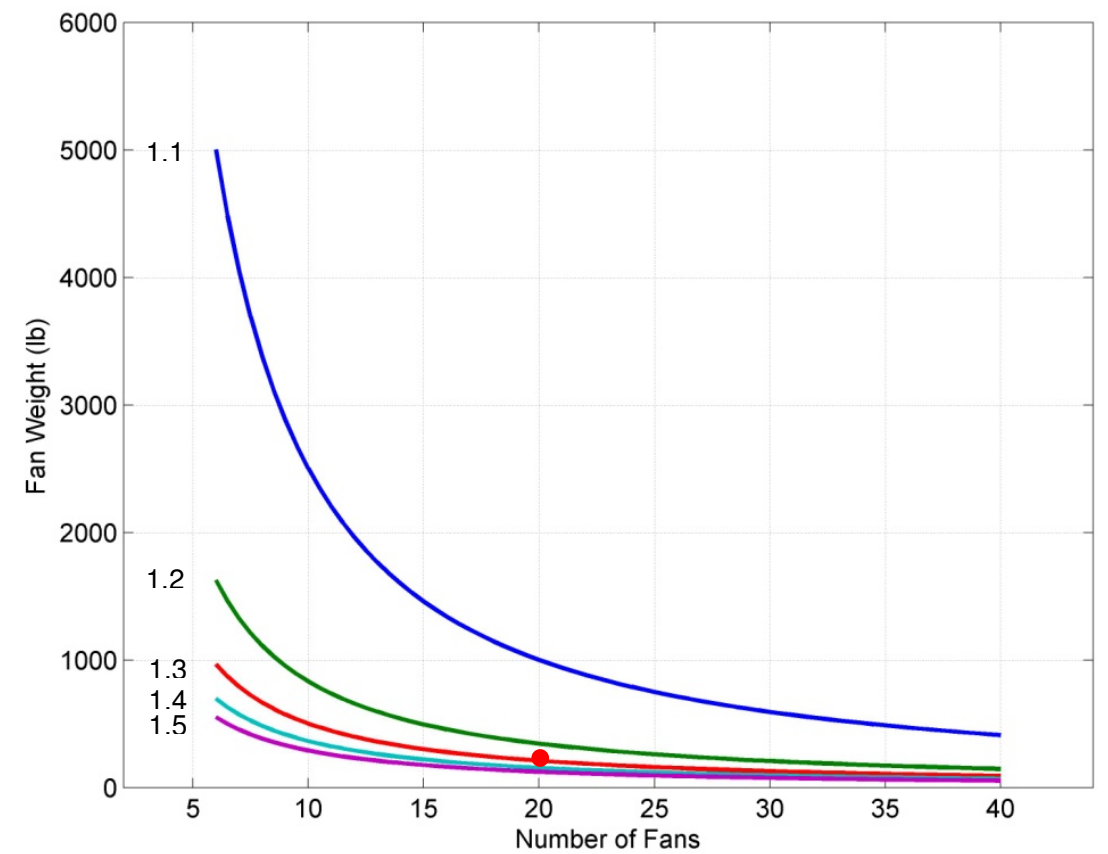

Figure 78. Fan Weight versus Number of Fans and Fan Pressure Ratio. 
The weight of the fan depends mostly on the diameter of the fan. Higher pressure ratios lead to smaller diameter and thus lighter fans. Smaller fans however operate at higher speeds, increasing the disc stress and disc weight. Figure 77 reveals that inlet pressure recovery has a significant impact on fan weight at low fan pressure ratios, but little impact at high pressure ratios. Figure 78 shows that increasing the number of fans above 20 would yield negligible reductions in individual fan weight.

\subsubsection{D Fan Analysis}

In addition to the $1 \mathrm{D}$ fan performance, the user may also be interested in the 2D performance of the fan. The only input for the 2D performance is the air inlet angle to the fan which will typically be zero unless inlet guide vanes are installed. The other parameters required for the 2D fan analysis come from the 1D fan and weight analyses. Table 12 shows the output of the 2D fan analysis for this aircraft at the on-design condition.

Table 12. On-Design 2D Fan Performance Outputs.

\begin{tabular}{|l|c|c|}
\hline \multicolumn{1}{|c|}{ Output } & Units & Value \\
\hline Absolute velocity at rotor inlet & $\mathrm{ft} / \mathrm{s}$ & 591 \\
\hline Absolute velocity at rotor exit & $\mathrm{ft} / \mathrm{s}$ & 681 \\
\hline Axial velocity at rotor inlet & $\mathrm{ft} / \mathrm{s}$ & 591 \\
\hline Axial velocity at rotor exit & $\mathrm{ft} / \mathrm{s}$ & 591 \\
\hline Whirl velocity at rotor inlet & $\mathrm{ft} / \mathrm{s}$ & 0.0 \\
\hline Whirl velocity at rotor exit & $\mathrm{ft} / \mathrm{s}$ & 337 \\
\hline Relative velocity at rotor inlet & $\mathrm{ft} / \mathrm{s}$ & 872 \\
\hline Relative velocity at rotor exit & $\mathrm{ft} / \mathrm{s}$ & 665 \\
\hline Absolute air inlet angle & $\circ$ & 0.0 \\
\hline Absolute air exit angle & $\circ$ & 29.7 \\
\hline Relative air inlet angle & $\circ$ & 47.3 \\
\hline Relative air exit angle & $\circ$ & 27.2 \\
\hline Deflection angle & $\circ$ & 20.1 \\
\hline Stall deflection angle & $\circ$ & 25.2 \\
\hline Incidence angle & $\circ$ & 0.0 \\
\hline Deviation angle & $\circ$ & 4.86 \\
\hline Camber angle & $\circ$ & 25.0 \\
\hline Stagger angle & $\circ$ & 34.8 \\
\hline Mean fan diameter & $\circ$ & 0.76 \\
\hline Mean fan blade speed & $\mathrm{ft} / \mathrm{s}$ & 641 \\
\hline Fan blade tip speed & $\mathrm{ft} / \mathrm{s}$ & 985 \\
\hline Relative inlet Mach number & - & 0.85 \\
\hline Relative tip Mach number & - & 1.13 \\
\hline de Haller number & - & 0.76 \\
\hline NACA diffusion factor & - & 0.43 \\
\hline Degree of reaction & - & 0.74 \\
\hline
\end{tabular}


The resulting flow velocities and angles in this table have been drawn onto a velocity triangle diagram in Figure 79. Velocities in this figure are in $\mathrm{ft} / \mathrm{s}$.

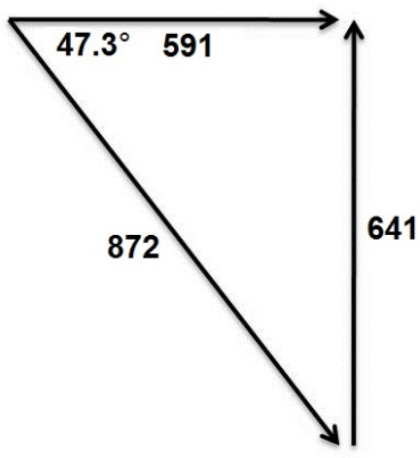

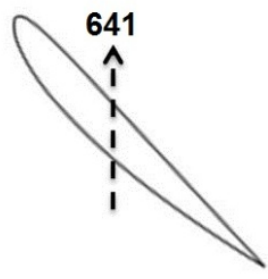

Rotor
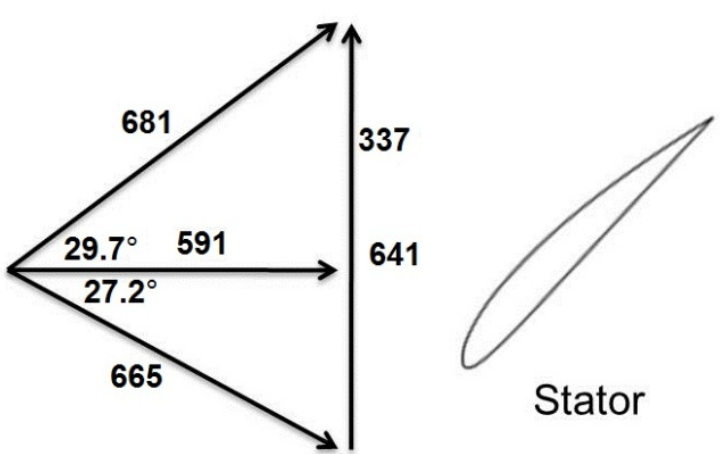

Figure 79. 2D Velocity Triangles.

According to the de Haller number and the NACA diffusion factor, the rotor appears to operate within reasonable limits and should have relatively low losses at the design point. The blade angles are also within reasonable limits. As before, the user may be interested in how these parameters are affected by the fan pressure ratio and inlet pressure recovery. In the $2 \mathrm{D}$ analysis however, the inlet pressure recovery does not have an effect on the results. This is because the inlet pressure recovery is merely an adjustment on total pressure, while the $2 \mathrm{D}$ analysis focuses on flow velocities and angles. Figure 80 shows the rotor exit flow angles and the required fluid deflection versus fan pressure ratio. The black dashed line in this figure indicates the on-design fan pressure ratio of 1.30 . 


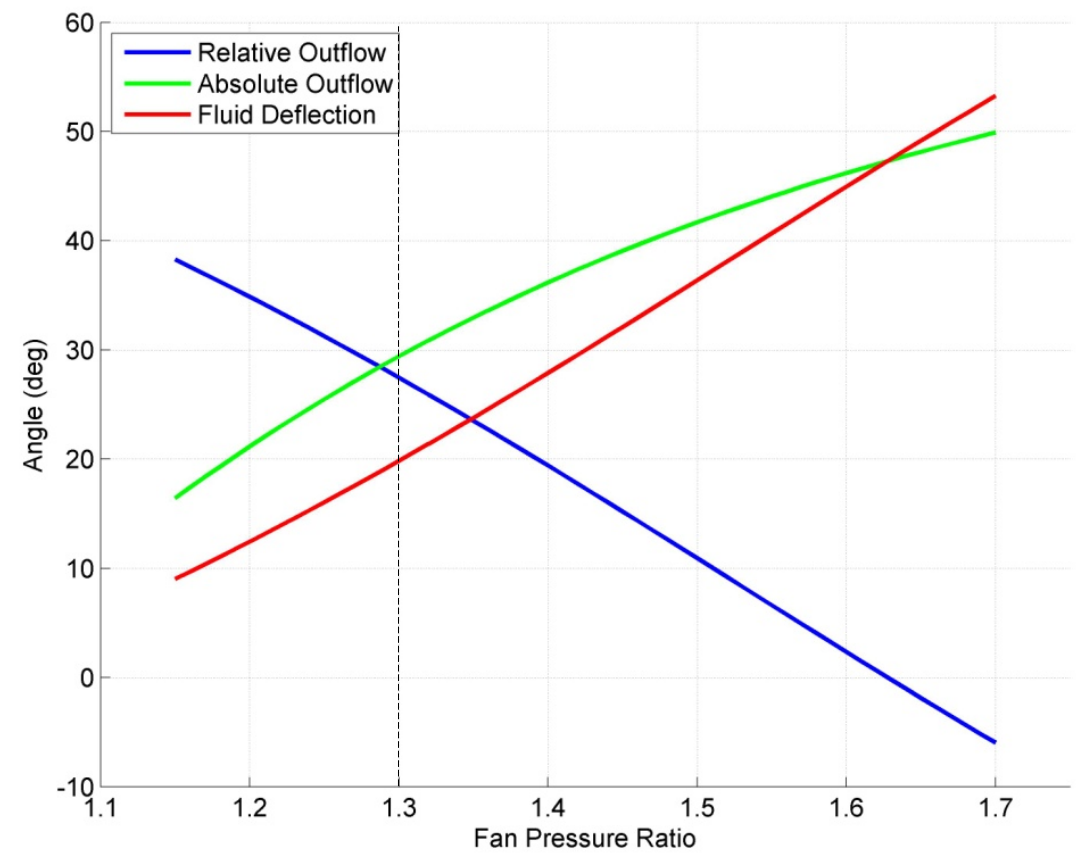

Figure 80. Flow Angles versus Fan Pressure Ratio.

This figure reveals that increasing fan pressure ratio requires an increase in fluid deflection. The higher the fan pressure ratio, the more enthalpy must to be added to the flow, which is accomplished by the rotor by deflecting the flow a greater amount. Greater fluid deflection results in a greater absolute exit angle and a reduced relative exit angle. Figure 81 shows the angles of the blades with respect to fan pressure ratio. 


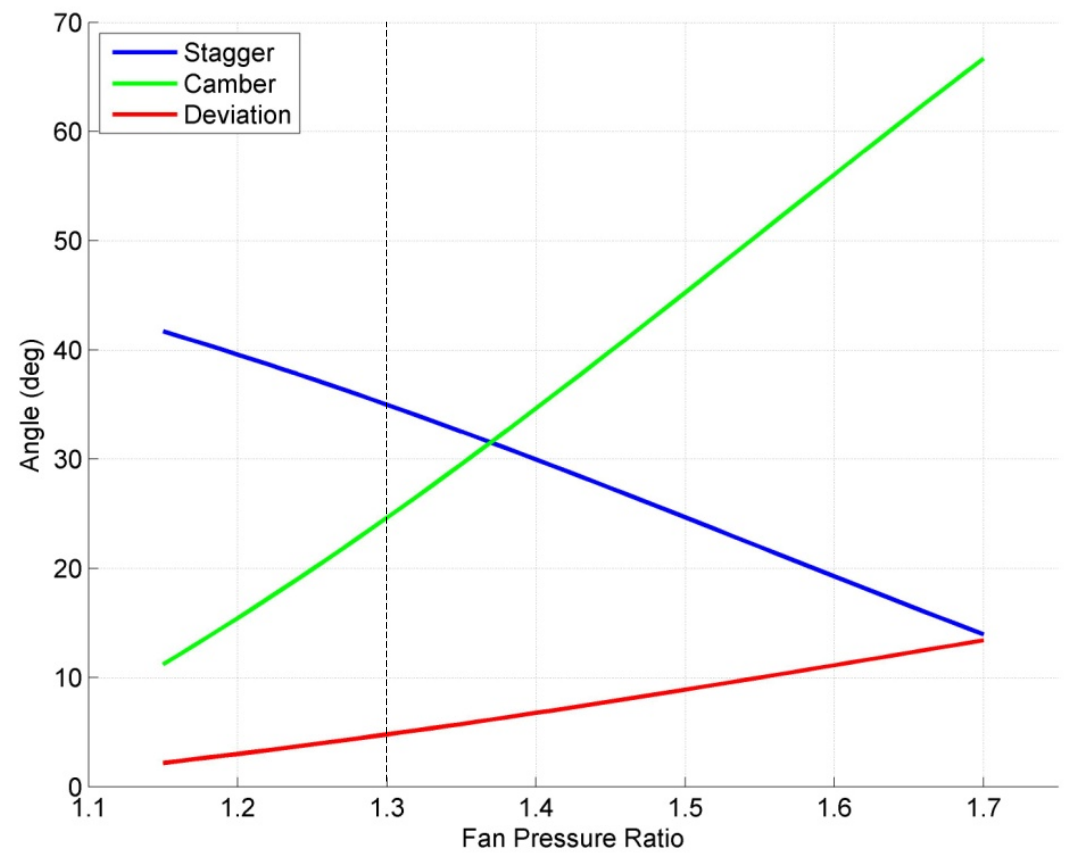

Figure 81. Blade Angles versus Fan Pressure Ratio.

This figure tells a similar story as the previous figure. In order to achieve the high fluid deflections required by high fan pressure ratios, the blade angles must be more extreme. The camber angle is the largest indicator of the severe blade angles that would be required. As the camber angle increases the deviation angle tends to increase as well. Finally, Figure 82 shows the two diffusion factors and the degree of reaction versus fan pressure ratio. 


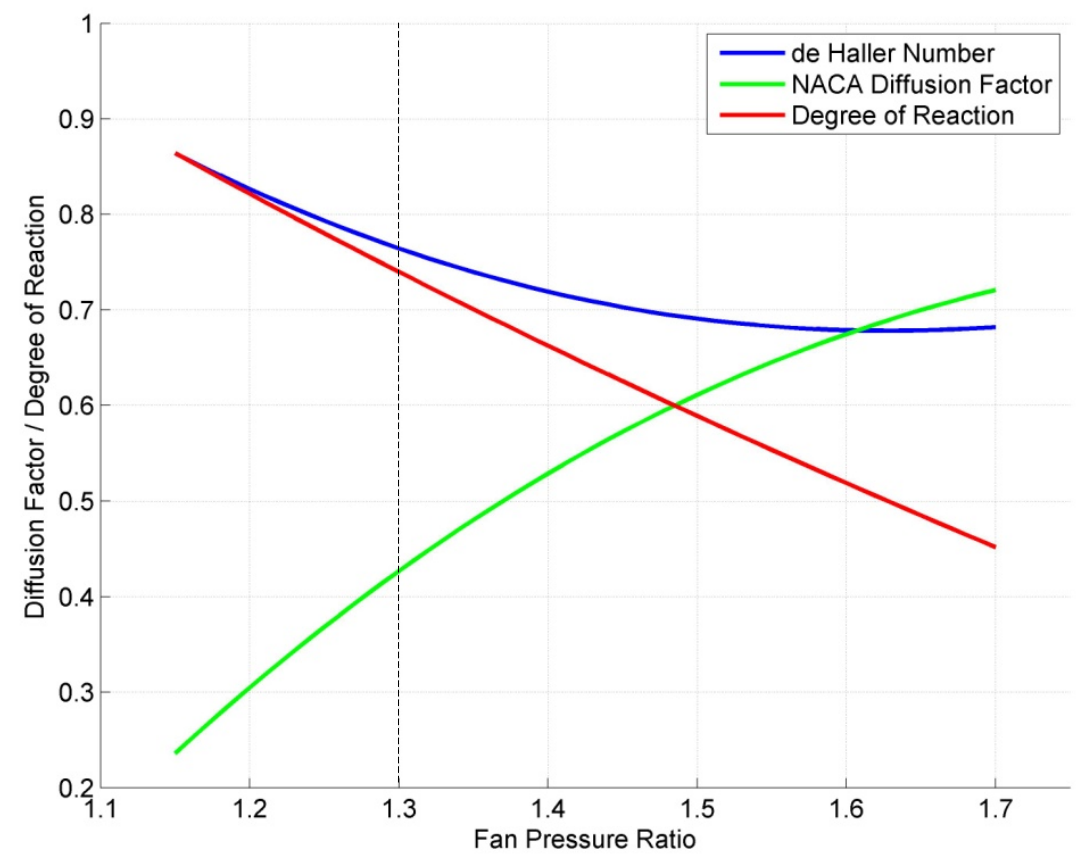

Figure 82. Diffusion Factor and Degree of Reaction versus Fan Pressure Ratio.

Recall from section 4.7.6, the de Haller number should be greater than 0.72 to avoid excessive diffusion in the rotor. This figure reveals that fan pressure ratios over 1.4 would result in excessive diffusion according to the de Haller number. In addition, the NACA diffusion factor should be less than approximately 0.5 at the mean fan diameter to again avoid losses. This occurs at fan pressure ratios above 1.37. Both of these predictors for rotor diffusion offer similar results with the NACA diffusion factor being slightly more pessimistic. To reduce diffusion, the fan tip Mach number can be increased at these higher fan pressure ratios. This also alleviates the need for such severe blade and flow angles.

Lastly, the degree of reaction reveals how much the rotor contributes to the overall enthalpy rise in the stage compared to the stator. High fan pressure ratios appear to require a lower degree of reaction, or in other words the stator must contribute more heavily to the enthalpy rise. Low degree of reaction rotors tend to have very high camber angles which agrees with the trend in Figure 81. 


\subsubsection{Electrical System Analysis}

The next aspect of this vehicle's hybrid propulsion system that will be considered is the electrical system. Table 13 lists the inputs to the on-design electrical system analysis that was used for this example.

Table 13. On-Design Electrical System Inputs.

\begin{tabular}{|l|c|c|}
\hline \multicolumn{1}{|c|}{ Input } & Units & Value \\
\hline Generator sizing function & - & @motogensize_remy \\
\hline Motor sizing function & - & @motogensize_remy \\
\hline Generator weight function & - & @motorgenwgt_Pwr \\
\hline Motor weight function & - & @motorgenwgt_Pwr \\
\hline Generator diameter & $\mathrm{ft}$ & 1.5 \\
\hline Generator speed & RPM & 8000 \\
\hline Motor diameter increase & $\%$ & 0.0 \\
\hline Generator efficiency function & - & @remy_hvh25090P700_eta \\
\hline Motor efficiency function & - & @remy_hvh25090P700_eta \\
\hline Generator performance function & - & @remy_hvh25090P700_perf \\
\hline Generator performance function & - & @remy_hvh25090P700_perf \\
\hline Generator speed fraction & - & 0.55 \\
\hline Generator torque fraction & - & 0.40 \\
\hline Motor speed fraction & - & 0.60 \\
\hline Motor torque fraction & - & 0.40 \\
\hline Controller efficiency & $\%$ & 96.0 \\
\hline Battery efficiency & $\%$ & 99.0 \\
\hline Battery output power & $\mathrm{hp}$ & 0.0 \\
\hline
\end{tabular}

For the electrical analysis, the physical dimensions of the motors and generators will be based on the volumetric torque density of the Remy HVH250 shown previously in Figure 51 . The motor and generator weight will be determined by the weight versus power curve shown in Figure 58. The efficiency map of this Remy motor and its power and torque curves were also used in this analysis for both the motors and generators. Each of these aspects of the machine design is inputted as a function which the user can create easily for other machine types.

At the design point the battery will not be charging or discharging. The diameter and speed of the generator are inputted by the user, while the motor diameter is given as a percentage increase or decrease over the fan hub diameter. In this case the motor diameter will be equal to the fan diameter. The outputs from the on-design electrical system analysis are shown here in Table 14. These outputs are defined per motor/generator. 
Table 14. On-Design Electrical System Outputs.

\begin{tabular}{|c|c|c|}
\hline Output & Units & Value \\
\hline Motor diameter & $\mathrm{ft}$ & 0.79 \\
\hline Motor length & $\mathrm{ft}$ & 5.16 \\
\hline Generator length & $\mathrm{ft}$ & 13.97 \\
\hline Motor speed & RPM & 7168 \\
\hline Motor maximum speed & RPM & 11947 \\
\hline Generator maximum speed & RPM & 14545 \\
\hline Controller input power & $\mathrm{hp}$ & 19238 \\
\hline Controller output power & $\mathrm{hp}$ & 18468 \\
\hline Generator input power & $\mathrm{hp}$ & 10118 \\
\hline Generator output power & hp & 9619 \\
\hline Generator efficiency & $\%$ & 95.1 \\
\hline Generator torque & $\mathrm{ft}^{\mathrm{ft}} \mathrm{lb}_{\mathrm{f}}$ & 6315 \\
\hline Generator power density & $\mathrm{hp} / \mathrm{lb}$ & 5.07 \\
\hline Generator torque density & $\mathrm{ft}-\mathrm{Ib}_{\mathrm{f}} / \mathrm{lb}$ & 3.98 \\
\hline Motor input power & $\mathrm{hp}$ & 923 \\
\hline Motor output power & $\mathrm{hp}$ & 877 \\
\hline Motor efficiency & $\%$ & 94.9 \\
\hline Motor torque & $\mathrm{ft}-\mathrm{lb}_{\mathrm{f}}$ & 643 \\
\hline Motor power density & $\mathrm{hp} / \mathrm{lb}$ & 2.65 \\
\hline Motor torque density & $\mathrm{ft}-\mathrm{lb}_{\mathrm{f}} / \mathrm{lb}$ & 2.53 \\
\hline Generator speed fraction for max peak power & - & 0.55 \\
\hline Generator speed fraction for max cont power & - & 0.55 \\
\hline Motor speed fraction for max peak power & - & 0.55 \\
\hline Motor speed fraction for max cont power & - & 0.55 \\
\hline Generator torque fraction for max peak power & - & 0.84 \\
\hline Generator torque fraction for max cont power & - & 0.66 \\
\hline Motor torque fraction for max peak power & - & 0.84 \\
\hline Motor torque fraction for max cont power & $\overline{-}$ & 0.66 \\
\hline Generator max peak power & $\mathrm{hp}$ & 20122 \\
\hline Generator max peak torque & $\mathrm{ft}^{\mathrm{l}-\mathrm{lb}}$ & 15787 \\
\hline Motor max peak power & $\mathrm{hp}$ & 1682 \\
\hline Motor max peak torque & $\mathrm{ft}^{\mathrm{l}-\mathrm{lb}}$ & 1607 \\
\hline Generator percent of max peak power & $\%$ & 47.8 \\
\hline Generator percent of max cont power & $\%$ & 61.4 \\
\hline Motor percent of max peak power & $\%$ & 53.5 \\
\hline Motor percent of max cont power & $\%$ & 67.7 \\
\hline Generator weight & $\mathrm{lb}$ & 3969 \\
\hline Generator controller weight & $\mathrm{lb}$ & 1191 \\
\hline Motor weight & $\mathrm{lb}$ & 634 \\
\hline Motor controller weight & $\mathrm{lb}$ & 190 \\
\hline Generator heat transfer rate & $\mathrm{BTU} / \mathrm{s}$ & 353 \\
\hline Motor heat transfer rate & BTU/s & 32.7 \\
\hline Total controller heat transfer rate & BTU/s & 769 \\
\hline Battery heat transfer rate & $\mathrm{BTU} / \mathrm{s}$ & 0.0 \\
\hline Total heat transfer rate & BTU/s & 2129 \\
\hline Total electrical system weight & $\mathrm{lb}$ & 26808 \\
\hline Overall system efficiency & $\%$ & 86.7 \\
\hline
\end{tabular}


The outputs from the on-design electric system analysis are quite extensive. This table shows the physical dimensions and weights of the electric machines, their speed, power, torque, as well as the machines operating point in relation to its maximum peak and maximum continuous power. These values were illustrated in Figure 57. This analysis also outputs the amount of heat each component is creating, the total system weight, and the overall system efficiency. For this aircraft, the electrical system weighs $26,808 \mathrm{lbs}$ with an efficiency of $86.7 \%$. This indicates that between the generator input shaft and the motor output shaft, $13.3 \%$ of the electrical power is lost. It can be safely assumed that all of these losses are converted to heat.

At the design point, the motors and generator are operating at $67.7 \%$ and $61.4 \%$ of maximum continuous power, respectively, indicating that they are oversized for this point. However, at the takeoff condition where much more power is available to the electrical system from the engines, these machines may be operating above continuous power. The user must be conscious of this behavior as it is unique to hybrid electric systems.

The user may also be interested in how the fan pressure ratio and inlet pressure recovery affect the electrical system. Figure 83 through Figure 86 show the motor and generator weight and lengths with respect to fan pressure ratio and inlet pressure recovery. The colored lines in these figures represent constant inlet pressure recovery as labeled in Figure 83. 


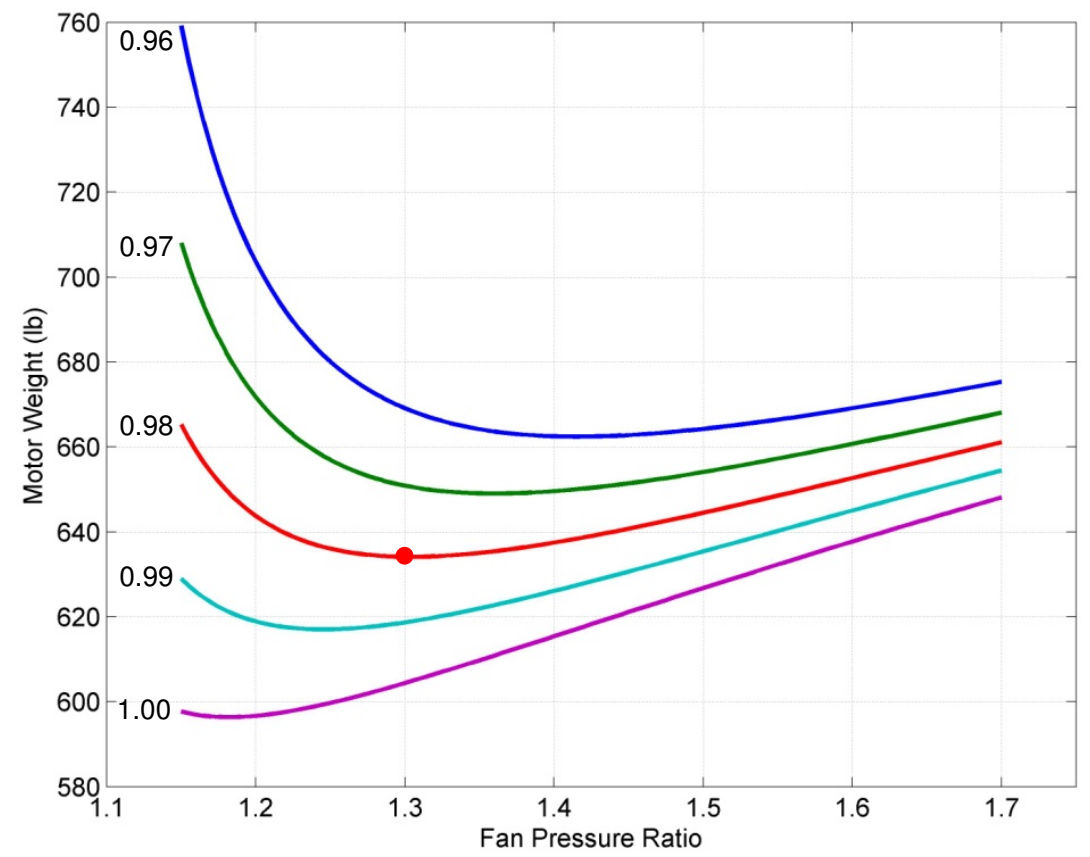

Figure 83. Motor Weight versus Fan Pressure Ratio and Inlet Pressure Recovery.

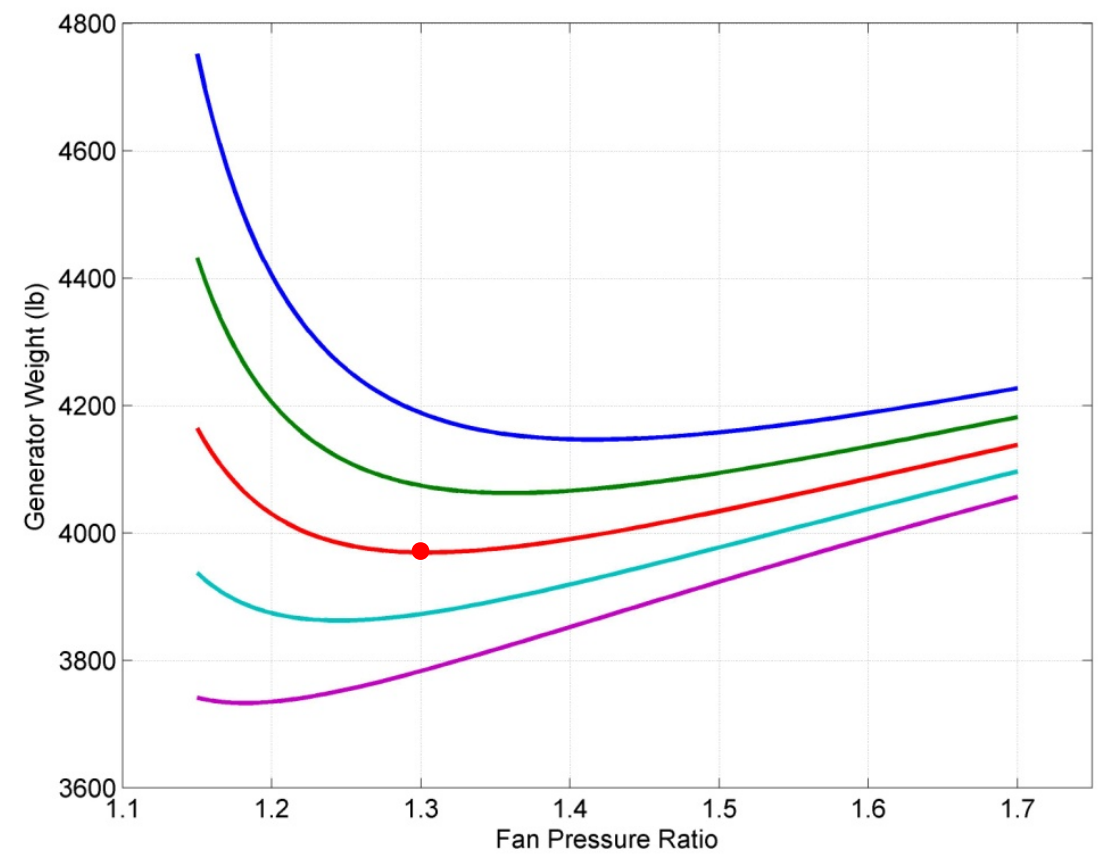

Figure 84. Generator Weight versus Fan Pressure Ratio and Inlet Pressure Recovery. 


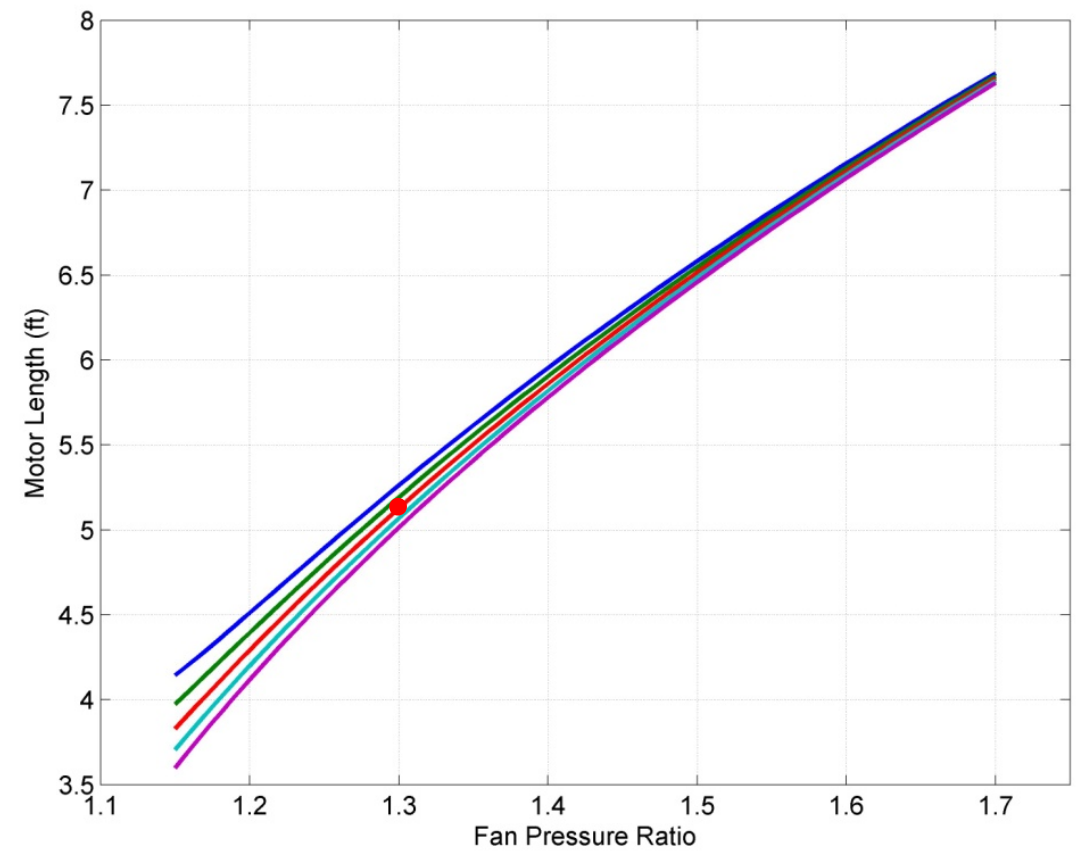

Figure 85. Motor Length versus Fan Pressure Ratio and Inlet Pressure Recovery.

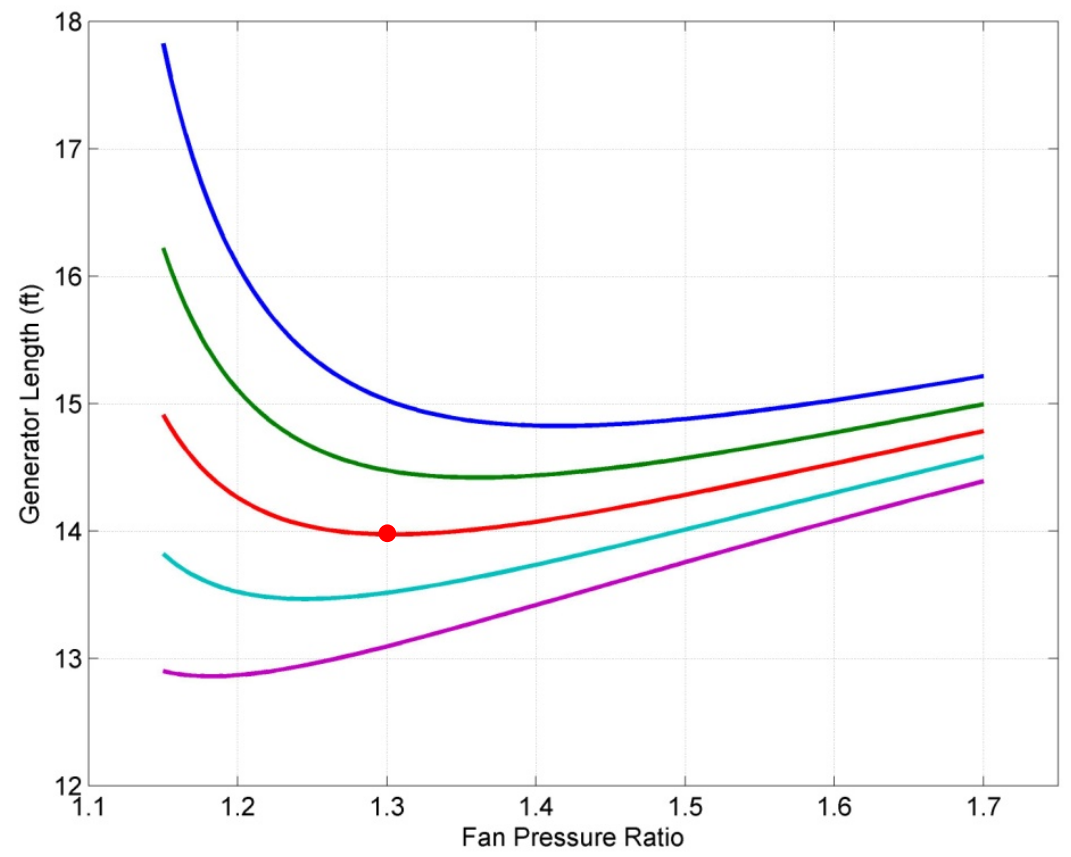

Figure 86. Generator Length versus Fan Pressure Ratio and Inlet Pressure Recovery. 
Figure 83 and Figure 84 show that the weight of the motors and generators has the same trend as the fan power in Figure 67. This is a result of the weight being determined by the weight versus power curve shown in Figure 58. The length of the generator in Figure 86 also has the same trend. The reason for this is that the generator's speed and diameter are fixed, and so length is added to achieve the correct machine volume and torque. The motor however varies in diameter and speed based on the fan design and thus the length increases with fan pressure ratio. Longer motors and generators can be difficult to integrate into the aircraft and is a concern for the designer.

\subsubsection{Engine Analysis}

The final aspect of the hybrid propulsion system that must be analyzed at the design point is the engine. This vehicle is equipped with two turboshaft engines which are sized to meet the power demand of the propulsion system at the design point. Table 15 shows the inputs to the on-design engine analysis for this example.

Table 15. On-Design Engine Inputs.

\begin{tabular}{|l|c|c|}
\hline \multicolumn{1}{|c|}{ Input } & Units & Value \\
\hline Number of engines & - & 2 \\
\hline Engine performance function & - & @honeywell_tpe33110 \\
\hline Flat rating? & - & No \\
\hline Engine weight function & - & @turbingwgt \\
\hline Fuel lower heating value & $\mathrm{BTU} / \mathrm{lb}_{\mathrm{m}}$ & 18400 \\
\hline Fuel-to-air ratio & - & 0.027 \\
\hline
\end{tabular}

There are far fewer inputs to the engine analysis than the other aspects of the propulsion system. In this example, the performance of the engine will be dictated by a function modeling the Honeywell TPE331-10 turboshaft engine. The power and fuel flow for this engine were shown in Figure 60. This engine will not be flat rated and its weight is determined by the curve-fit equation shown in Figure 64. The chosen lower heating value of the fuel is typical of Jet-A. The outputs of the engine analysis are shown in Table 16, defined per engine. 
Table 16. On-Design Engine Outputs.

\begin{tabular}{|l|c|c|}
\hline \multicolumn{1}{|c|}{ Output } & Units & Value \\
\hline Engine output power & $\mathrm{hp}$ & 10118 \\
\hline Engine max output power, SLS & $\mathrm{hp}$ & 20278 \\
\hline Power lapse & $\%$ & 49.9 \\
\hline PSFC & $\mathrm{Ib}_{\mathrm{m}} / \mathrm{hp}-\mathrm{hr}$ & 0.32 \\
\hline Fuel flow rate & $\mathrm{Ib}_{\mathrm{m}} / \mathrm{hr}$ & 3264 \\
\hline Air flow rate & $\mathrm{lb}_{\mathrm{m}} / \mathrm{s}$ & 33.3 \\
\hline Engine thermal efficiency & $\%$ & 42.9 \\
\hline Engine power density & $\mathrm{hp} / \mathrm{lb}$ & 9.13 \\
\hline Engine weight & $\mathrm{lb}$ & 2206 \\
\hline
\end{tabular}

\subsubsection{Overall System Performance}

Now that the entire hybrid propulsion system has been designed for this aircraft, some final overall performance metrics can be considered. The most important of these metrics is the TSFC, system thermal efficiency, propulsive efficiency, and the overall efficiency. These are listed in Table 17 for this example.

Table 17. On-Design Overall System Performance.

\begin{tabular}{|l|c|c|}
\hline \multicolumn{1}{|c|}{ Metric } & Units & Value \\
\hline TSFC & $\mathrm{Ib}_{\mathrm{m}} / \mathrm{l}_{\mathrm{f}}-\mathrm{hr}$ & 0.65 \\
\hline System thermal efficiency & $\%$ & 30.6 \\
\hline Propulsive efficiency & $\%$ & 85.8 \\
\hline Overall efficiency & $\%$ & 26.3 \\
\hline Effective bypass ratio & - & 24.9 \\
\hline
\end{tabular}

It can be seen that the TSFC for this vehicle is slightly higher compared to modern aircraft of similar sizes. For reference, a Boeing 737-700/800/900 powered by the CFM56-7B24 has a cruise TSFC of $0.627 \mathrm{Ib}_{\mathrm{m}} / \mathrm{lb}_{\mathrm{f}}$-hr. The TSFC listed in Table 17 is actually at the example vehicle's design point of top of climb. It is expected that the cruise TSFC will be several tenths greater.

The propulsive efficiency of this vehicle is exceptional due to the relatively low fan pressure ratio which is achievable with the use of several smaller fans instead of a few larger fans. Unfortunately, the thermal and overall efficiencies are poor due to the losses in the electrical system. 
As with the fan performance, fan weight, and electrical system performance, the user may again be interested in the effect of fan pressure ratio on the overall system performance. Figure 87 through Figure 91 show these performance metrics versus fan pressure ratio and inlet pressure recovery.

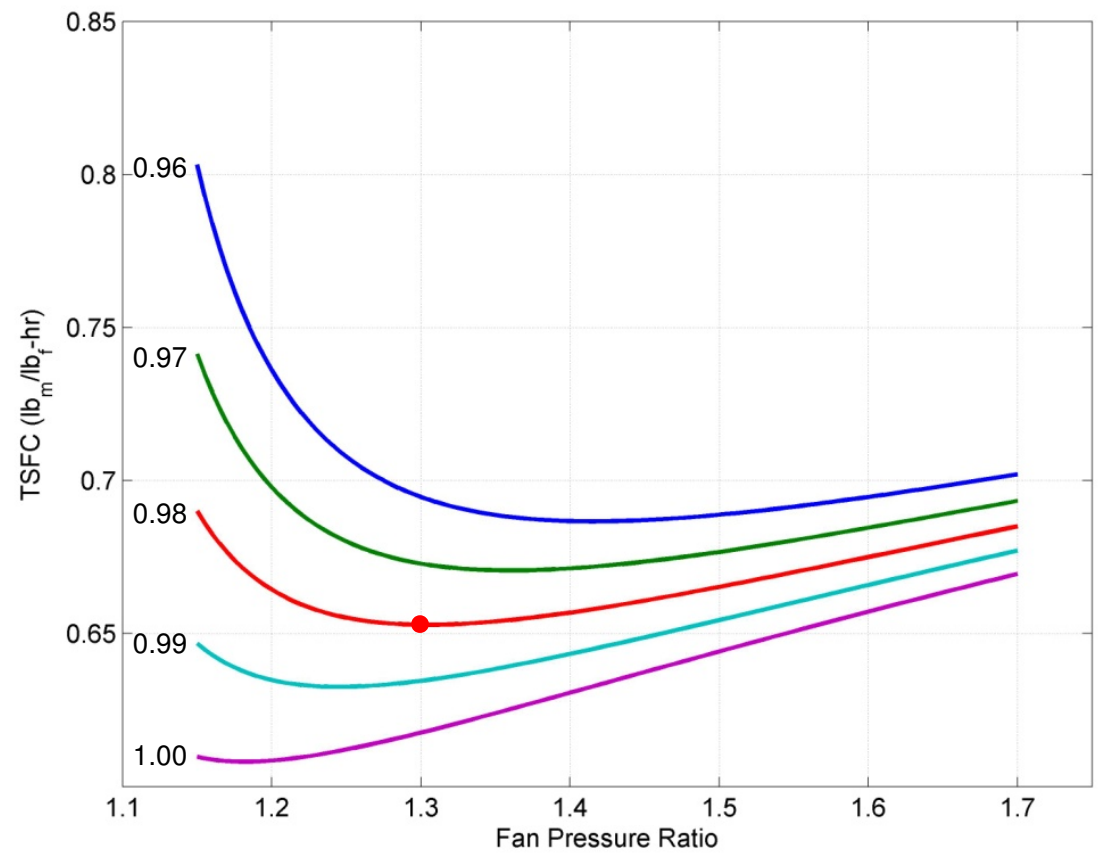

Figure 87. TSFC versus Fan Pressure Ratio and Inlet Pressure Recovery. 


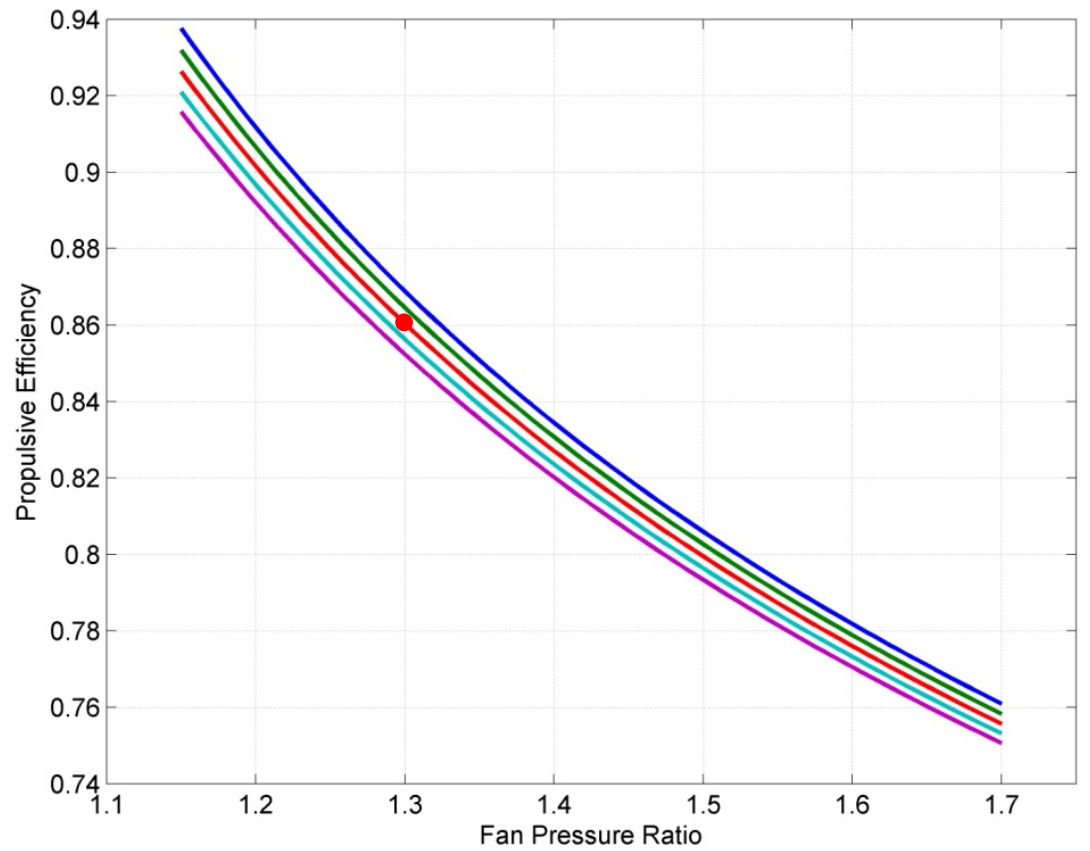

Figure 88. Propulsive Efficiency versus Fan Pressure Ratio and Inlet Pressure Recovery.

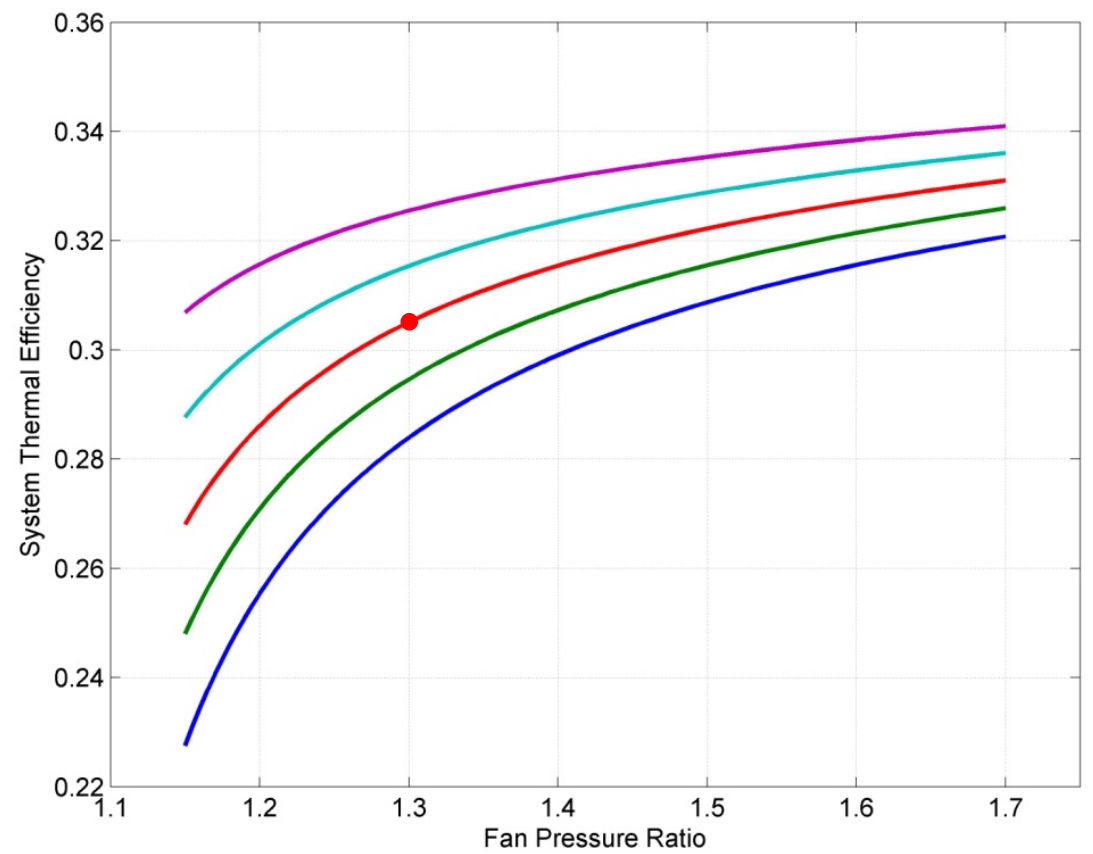

Figure 89. Thermal Efficiency versus Fan Pressure Ratio and Inlet Pressure Recovery. 


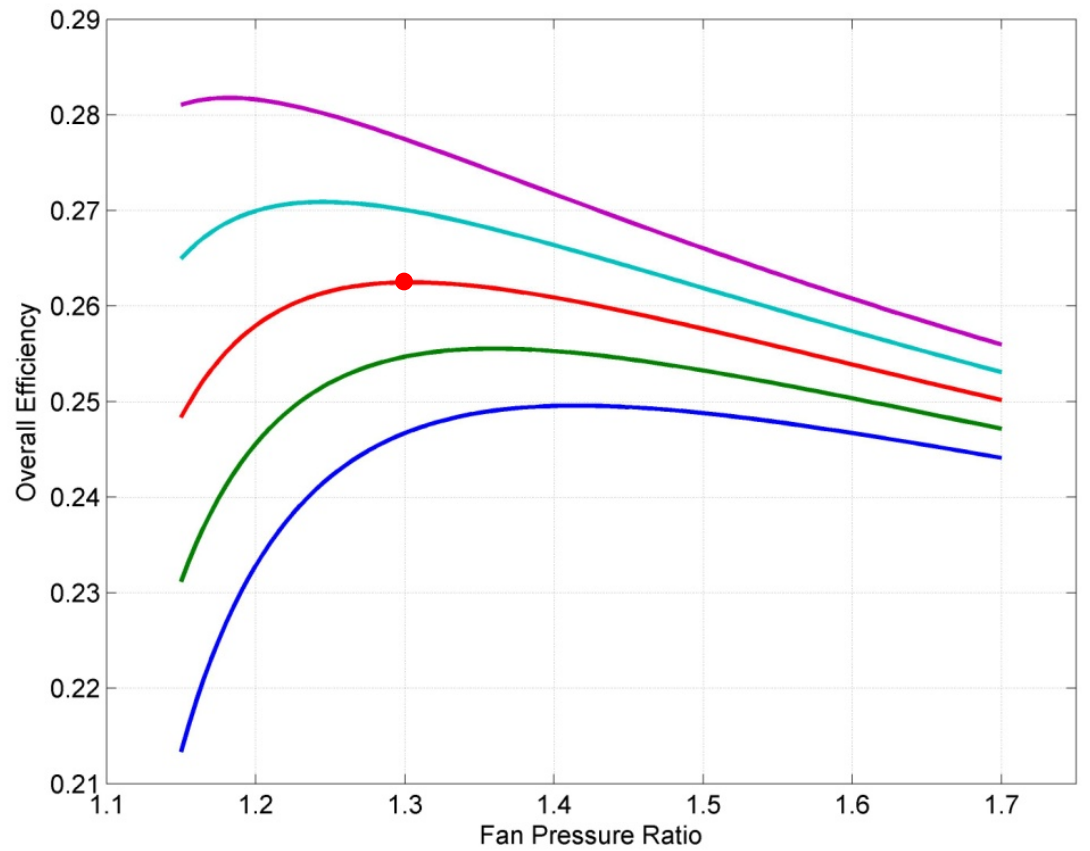

Figure 90. Overall Efficiency versus Fan Pressure Ratio and Inlet Pressure Recovery.

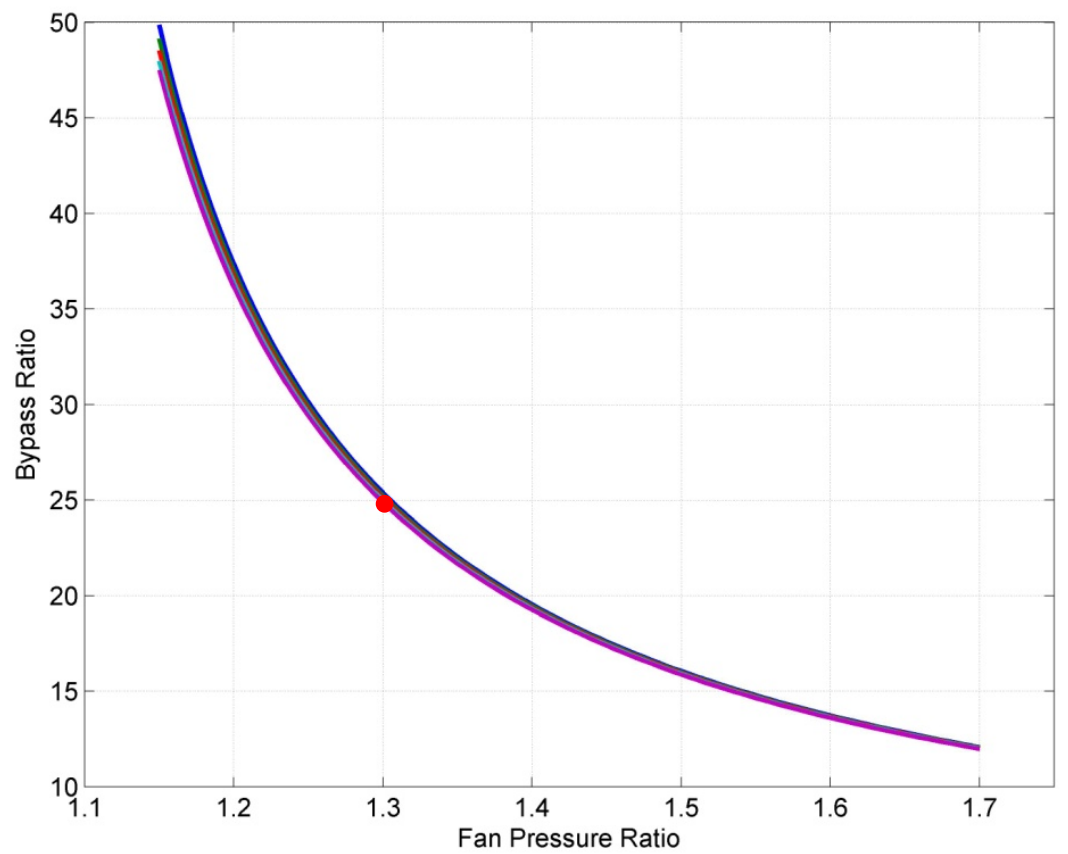

Figure 91. Bypass Ratio versus Fan Pressure Ratio and Inlet Pressure Recovery. 
Inlet pressure recovery and fan pressure ratio both have a significant impact on the TSFC of the aircraft. If the inlet pressure recovery were to improve by just $1 \%$ the TSFC could be reduced by about $3 \%$ at the same fan pressure ratio. The inlet pressure recovery does not have a large effect on propulsive efficiency while fan pressure ratio does. The system thermal efficiency tends to increase with fan pressure ratio because this leads to larger turbine engines which have a lower overall PSFC as illustrated in Figure 63. The overall efficiency is inversely related to the fan power trend in Figure 67. The fan pressure ratio for minimum fan power will produce the highest overall efficiency. Finally, the bypass ratio is greatly affected by the fan pressure ratio but not by the inlet pressure recovery. Very low fan pressure ratios can achieve very high bypass ratios but at the cost of increased weight and reduced TSFC.

A final important aspect of the hybrid propulsion system is the weight of the system. Figure 92 shows the system weight versus fan pressure ratio and inlet pressure recovery. This weight includes the fans, motors, generators, controllers, and turbine engines.

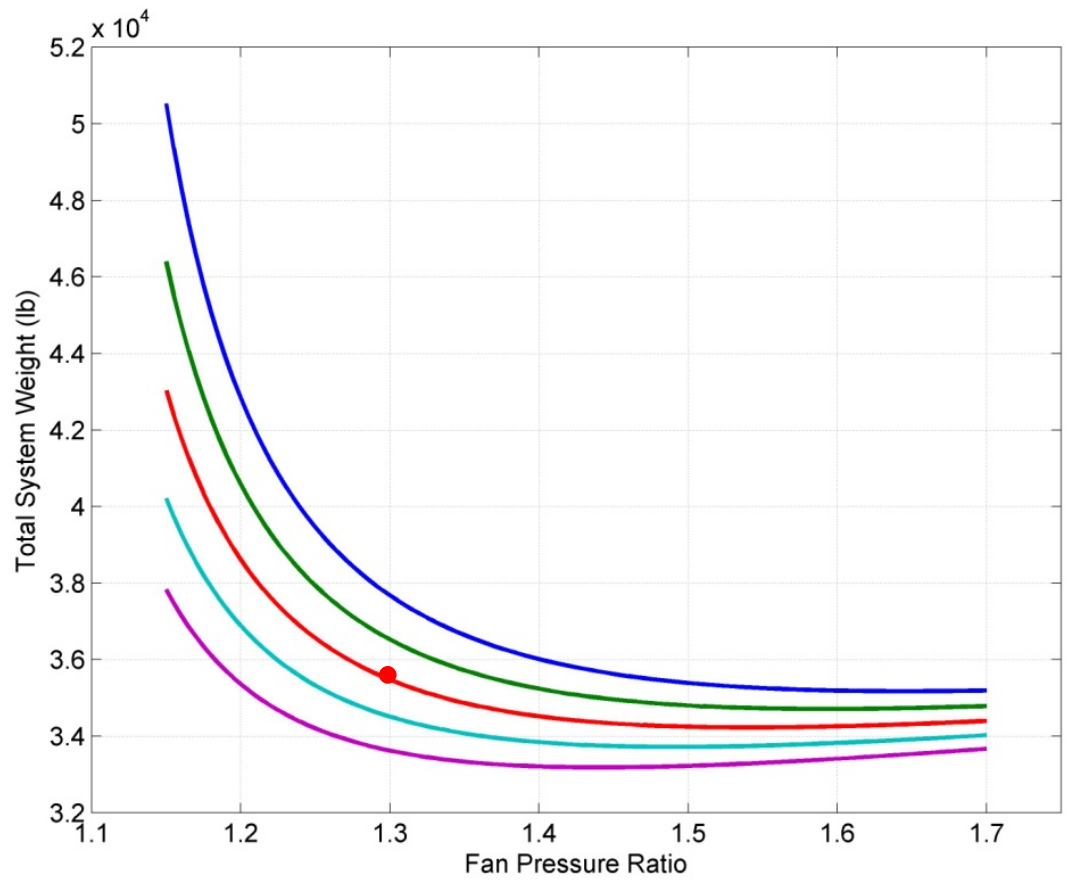

Figure 92. Total Weight versus Fan Pressure Ratio and Inlet Pressure Recovery.

This figure reveals that the weight of the propulsion system reaches a minimum value at some optimal fan pressure ratio. For an inlet pressure recovery of 0.98 (red line) the minimum 
weight occurs at a fan pressure ratio of approximately 1.55 . This example aircraft has a total propulsion system weight of $35,474 \mathrm{lbs}$, approximately 3 times the weight of two turbofan engines of comparable thrust. It is up to the user to balance the propulsion group weight and fuel efficiency whilst considering the design of the entire vehicle and its mission.

\subsection{Off-Design Analysis}

With the on-design analysis complete, the hybrid propulsion system has been sized to meet the requirements at top of climb. The user would now be interested in how the aircraft performs at off-design points such as cruise and takeoff. The off-design analysis is also important because aircraft mission simulation tools typically require tables of thrust, TSFC, and other propulsion system parameters. The user the easily create these tables with HAPSS. The offdesign analysis for this example will cover flight Mach numbers ranging from static to 0.8 and altitudes from sea level to 40,000 feet, all at $100 \%$ throttle. Mach numbers over 0.4 were not analyzed under 10,000 feet.

\subsubsection{Overall System Performance}

The overall system performance at the off-design points was considered first. Of the most interest to the user will be the thrust and the TSFC as these are the two main drivers for overall mission performance. The total thrust and TSFC are shown in Figure 93 and Figure 94, respectively. The colored lines represent constant altitude. 


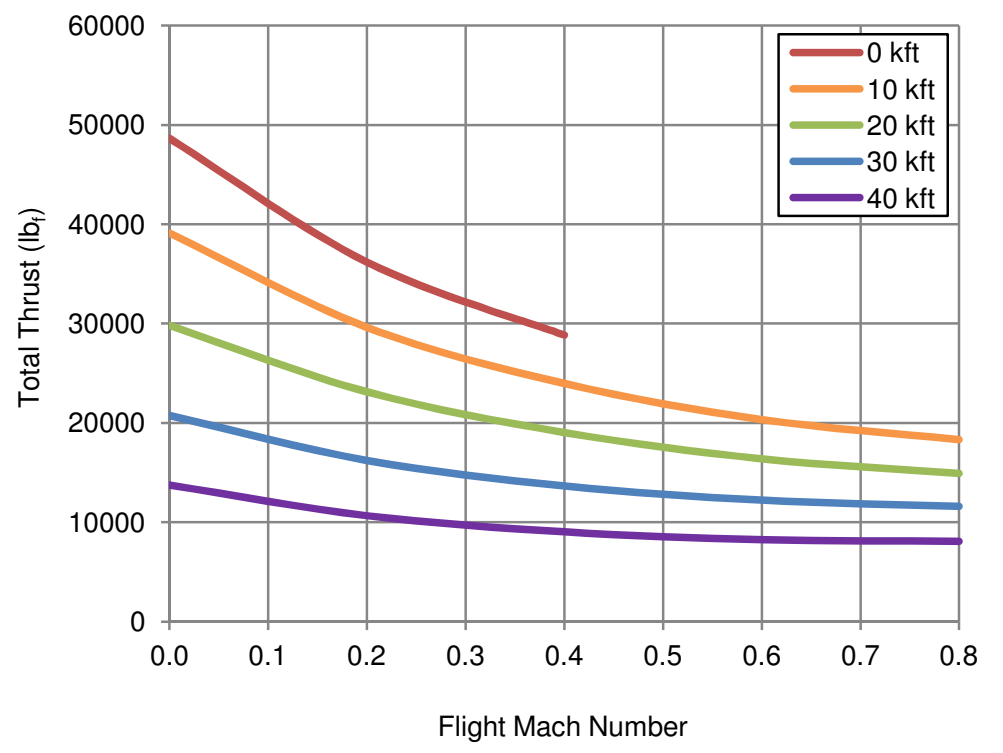

Figure 93. Total Thrust versus Flight Mach Number and Altitude.

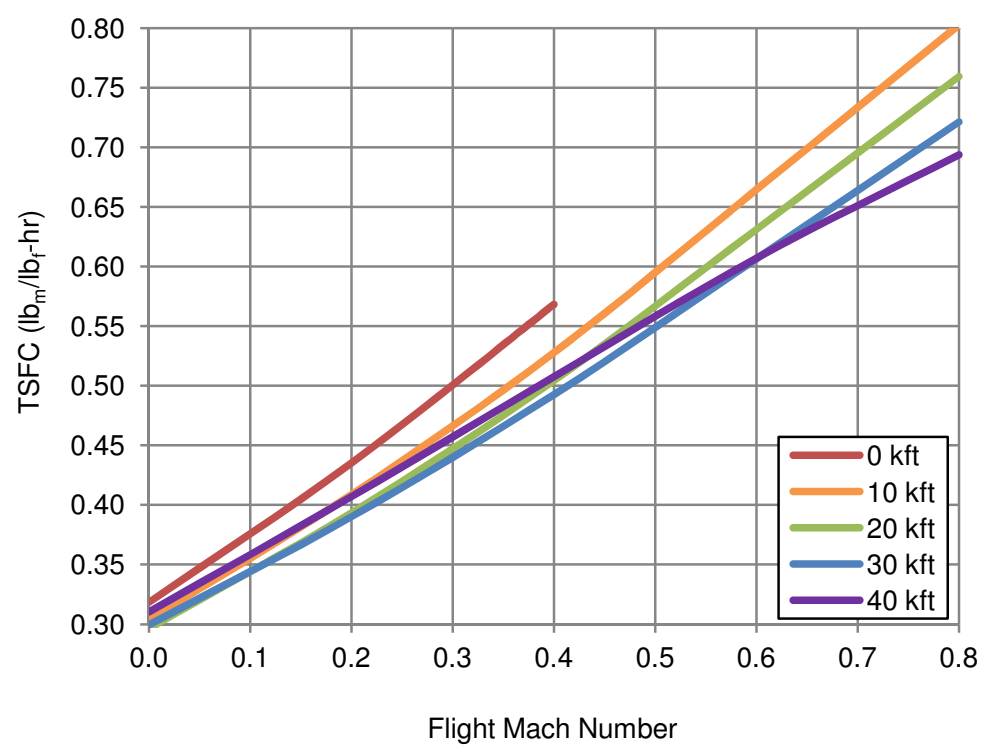

Figure 94. TSFC versus Flight Mach Number and Altitude.

The thrust of the vehicle decreases with flight speed and with altitude. The decrease in thrust with flight speed is more prevalent at lower altitudes however. This vehicle is able to achieve a sea level static thrust of $48,600 \mathrm{lb}_{\mathrm{f}}$. The TSFC of this vehicle steadily increases with flight speed and improves slightly with greater altitude except for at 40,000 feet where the TSFC begins to increase again. This odd behavior is a result of the engine PSFC being interpolated from a table of real engine data. This engine's unscaled PSFC was shown in Figure 62. 
Next, the propulsive, system thermal, and overall efficiencies of these vehicle were considered. These are shown in Figure 95 through Figure 97.

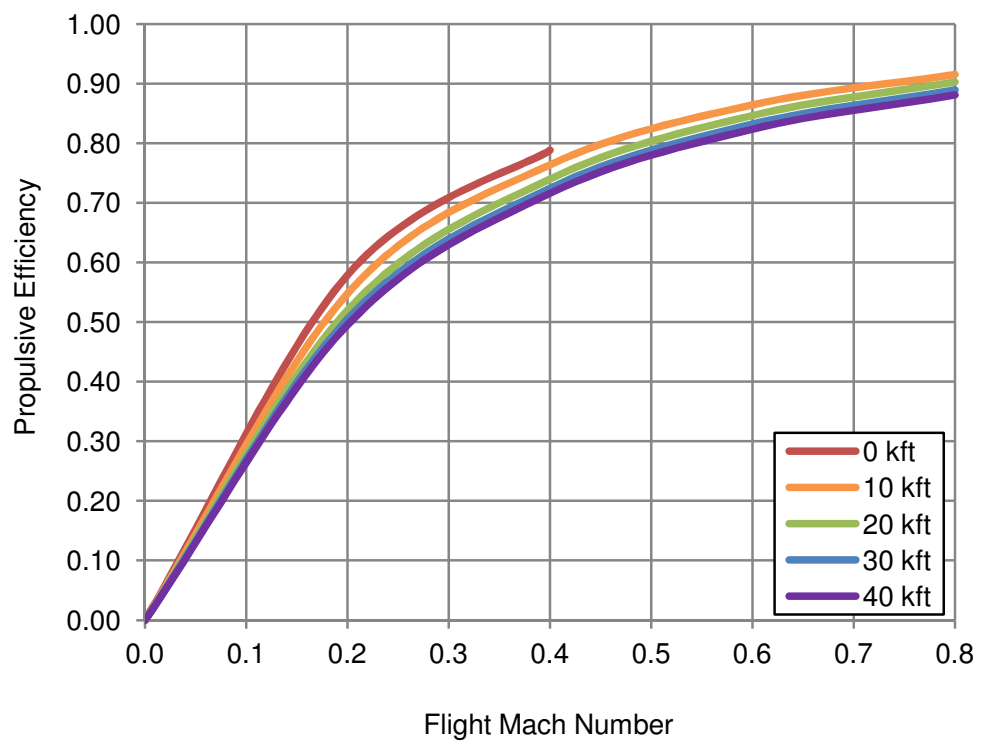

Figure 95. Propulsive Efficiency versus Flight Mach Number and Altitude.

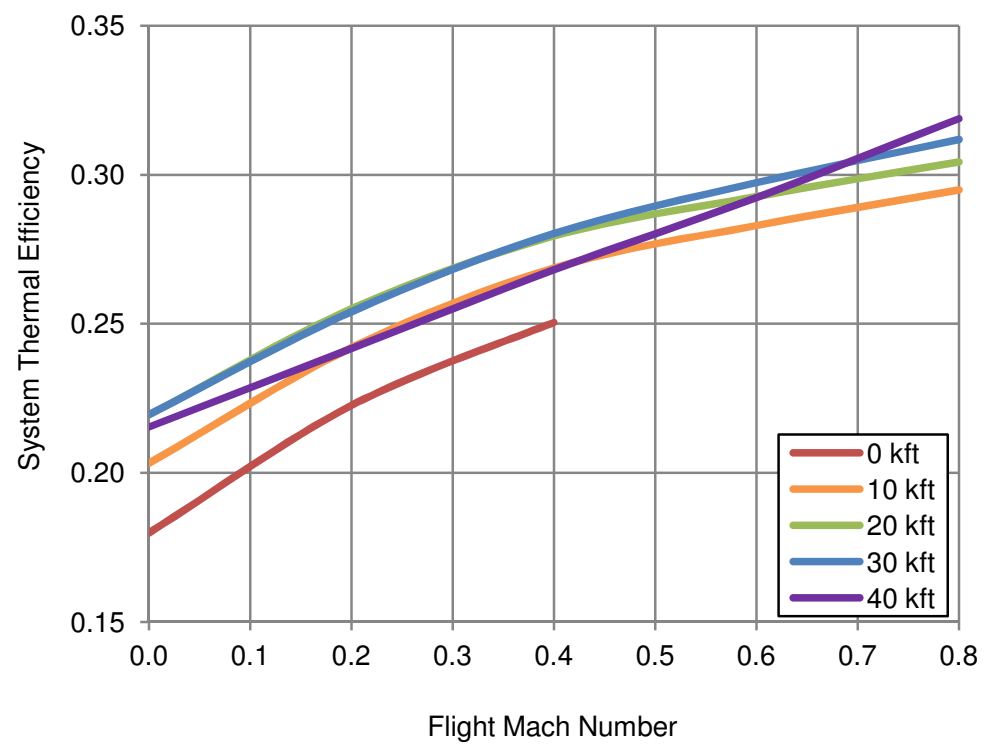

Figure 96. System Thermal Efficiency versus Flight Mach Number and Altitude. 


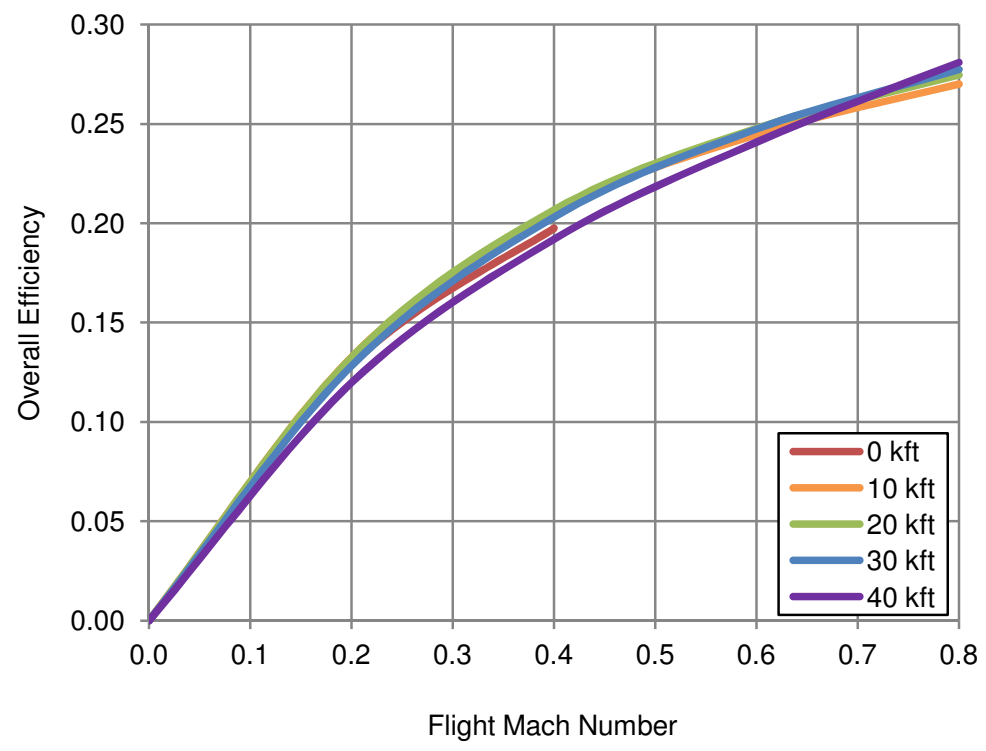

Figure 97. Overall Efficiency versus Flight Mach Number and Altitude.

It can be seen that the propulsive efficiency depends highly on the flight speed. Regardless, the propulsive efficiency at top of climb or cruise is generally of the most concern. The system thermal efficiency has a similar behavior as the TSFC as these parameters are directly related. Finally, the overall efficiency is the product of the propulsive and thermal efficiencies and peaks at $28.1 \%$, low compared to conventional turbofan engines.

\subsubsection{Engine Analysis}

In addition to the overall performance of the hybrid propulsion system, the user may also be interested in the performance of individual components such as the engine. Figure 98 shows the total engine power available while Figure 99 shows the PSFC of the engines. 


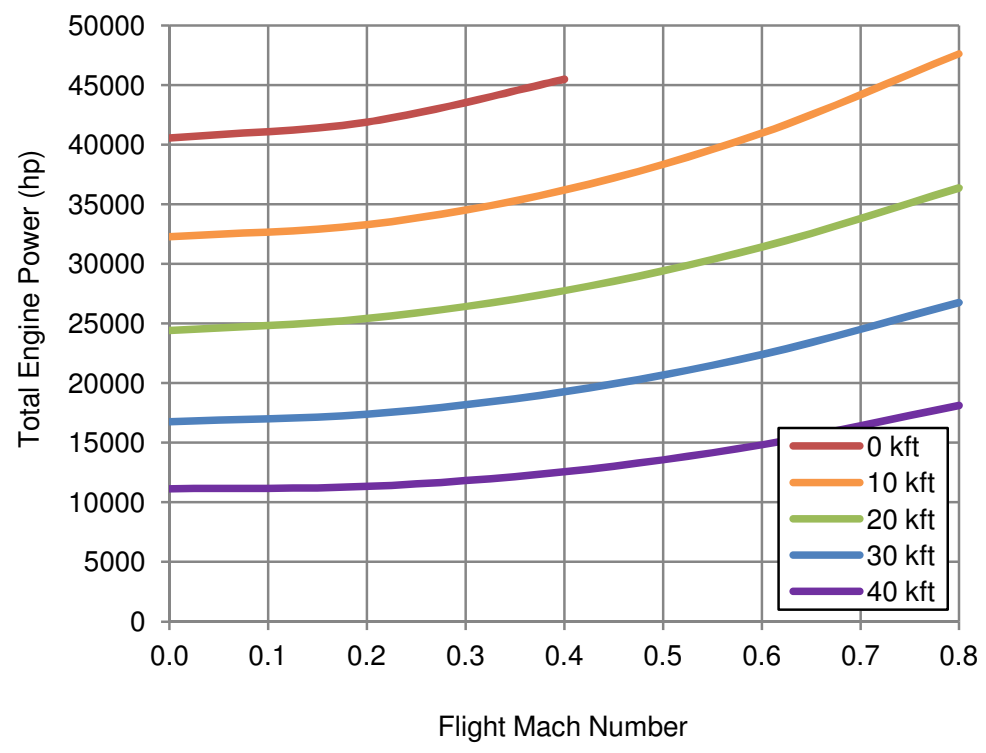

Figure 98. Total Engine Power versus Flight Mach Number and Altitude.

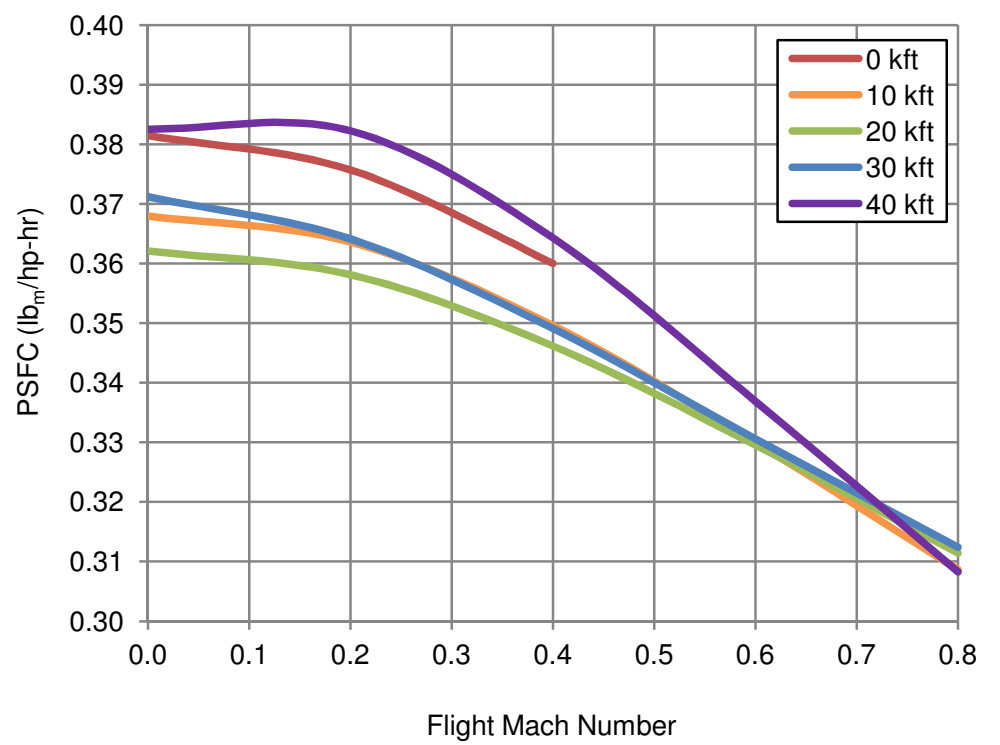

Figure 99. PSFC versus Flight Mach Number and Altitude.

The total power available from the engines increases with flight speed and decreases with altitude. This behavior can be seen in Figure 61 for this engine. Interestingly however, the total thrust decreases with flight speed despite the increase in power. The PSFC has generally decreasing trend with flight speed and again varies oddly with altitude due to the PSFC being interpolated from a table of real engine data. 


\subsubsection{Electrical System Analysis}

The user might also be interested in how the electrical system behaves at off-design conditions. Figure 100 through Figure 102 show the motor, generator, and electrical system efficiency for this aircraft in the off-design domain.

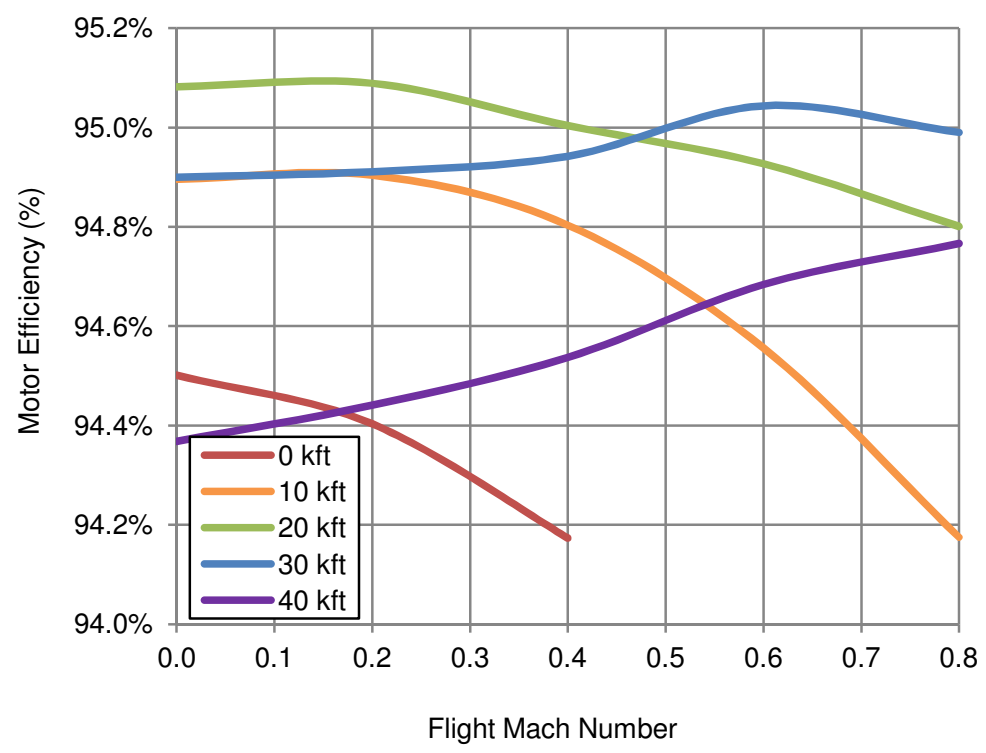

Figure 100. Motor Efficiency versus Flight Mach Number and Altitude.

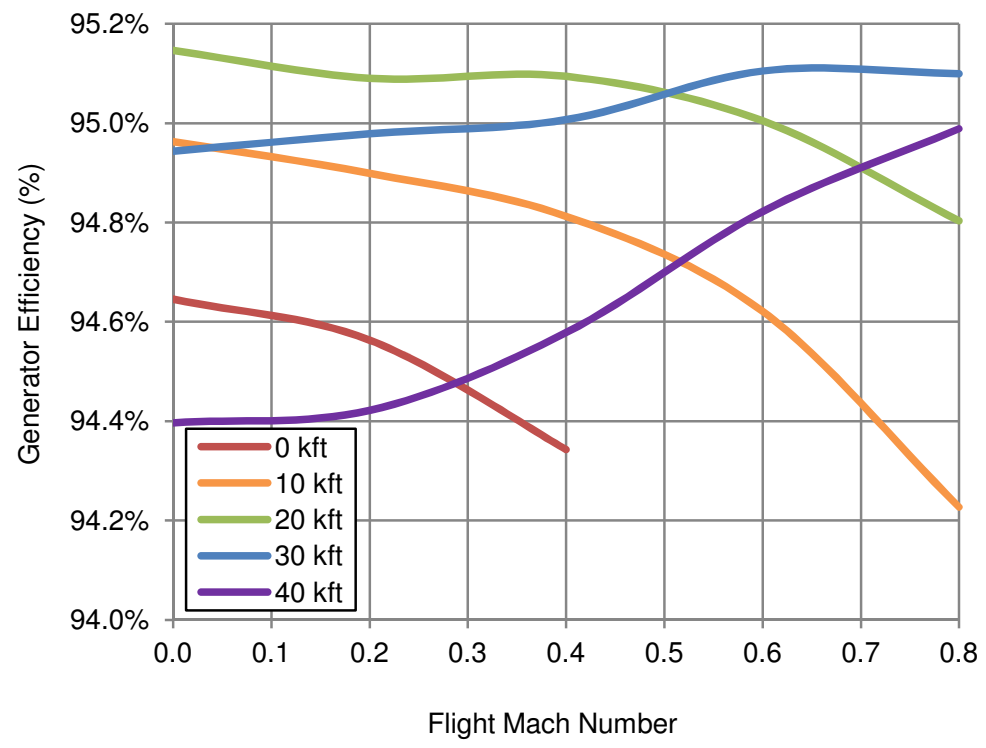

Figure 101. Generator Efficiency versus Flight Mach Number and Altitude. 


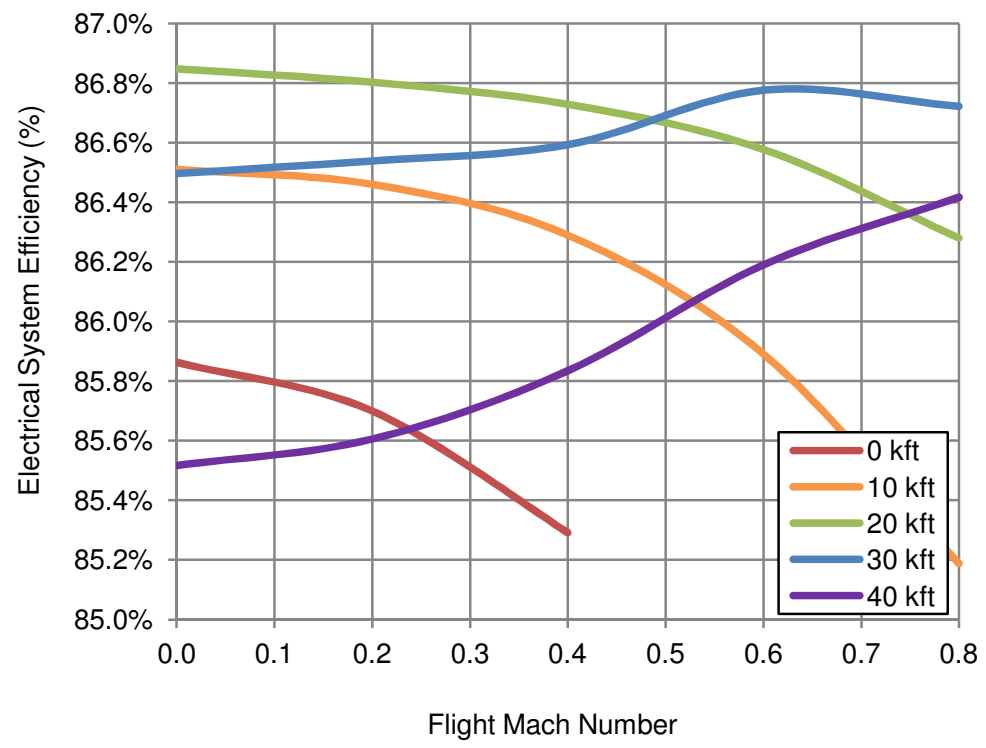

Figure 102. Electrical System Efficiency versus Flight Mach Number and Altitude.

At first glance these figures show seemingly chaotic efficiency trends for the motor and generator. However, these efficiencies are being interpolated from a map of real efficiency data and the behavior of these efficiencies can attributed to interpolation error as the operating point traverses the efficiency map. Furthermore, the efficiencies of the motor and generator only vary less than $1 \%$, indicating that these machines are kept within a fairly tight operating range despite the changing flight conditions. The electrical system efficiency is simply a product of the motor, generator, and controller efficiencies. This value is a direct indicator of the amount of power lost between the power producer and the thrust producer.

Next, the user may be interested in how much heat the electrical components are producing at any given flight condition. This will allow the user to notionally size a cooling system. Figure 103 through Figure 105 show the motor, generator, and controller heating in the off-design domain. 


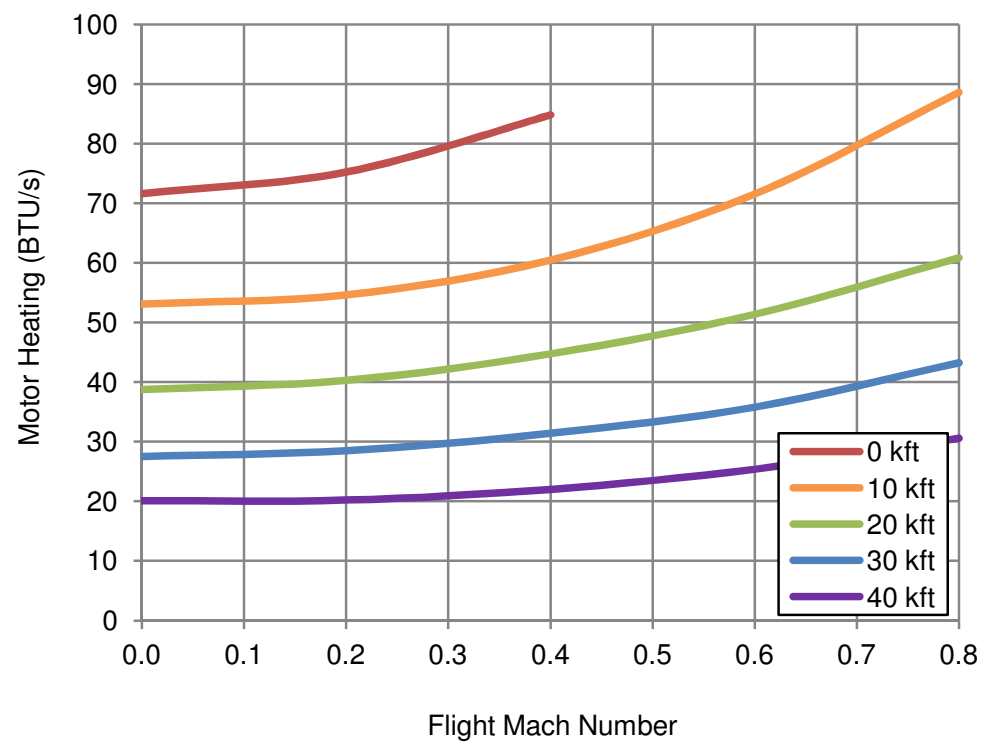

Figure 103. Motor Heating versus Flight Mach Number and Altitude.

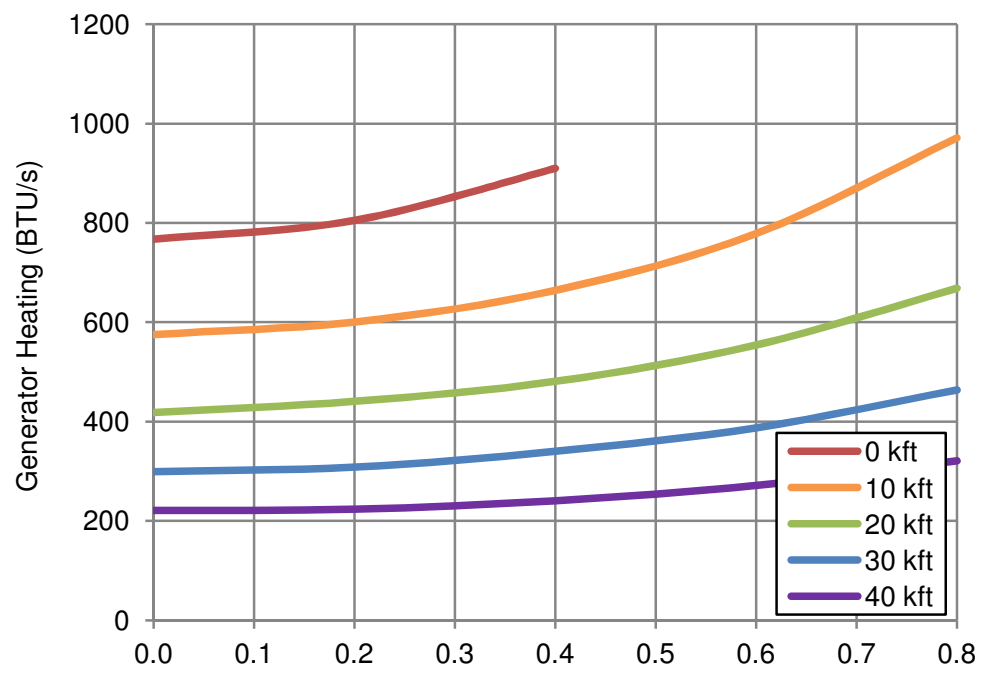

Flight Mach Number

Figure 104. Generator Heating versus Flight Mach Number and Altitude. 


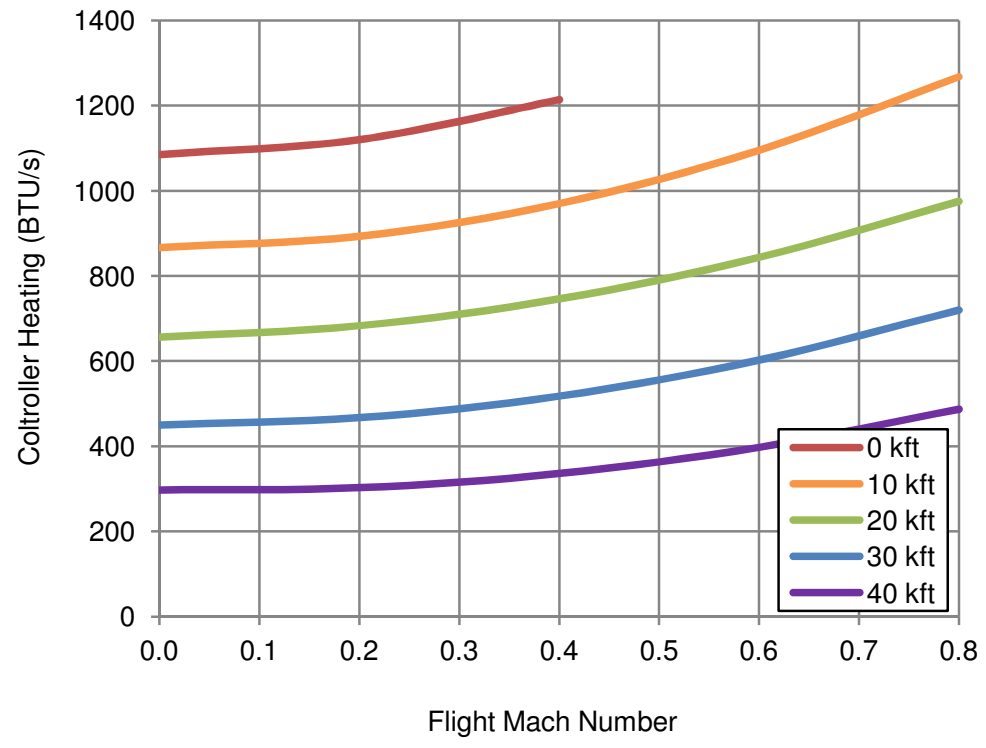

Figure 105. Controller Heating versus Flight Mach Number and Altitude.

The trends for the motor, generator, and controller heating are nearly identical to the total engine output power shown in Figure 98. This data reveals that at sea level static, the electrical system produces $4,059 \mathrm{BTU} / \mathrm{s}(4.28 \mathrm{MW})$ of waste heat that must be removed by a cooling system.

Lastly, the user may be interested in how closely the motors and generators operate to their maximum peak and maximum continuous power. This data is shown here in Figure 106 through Figure 109.

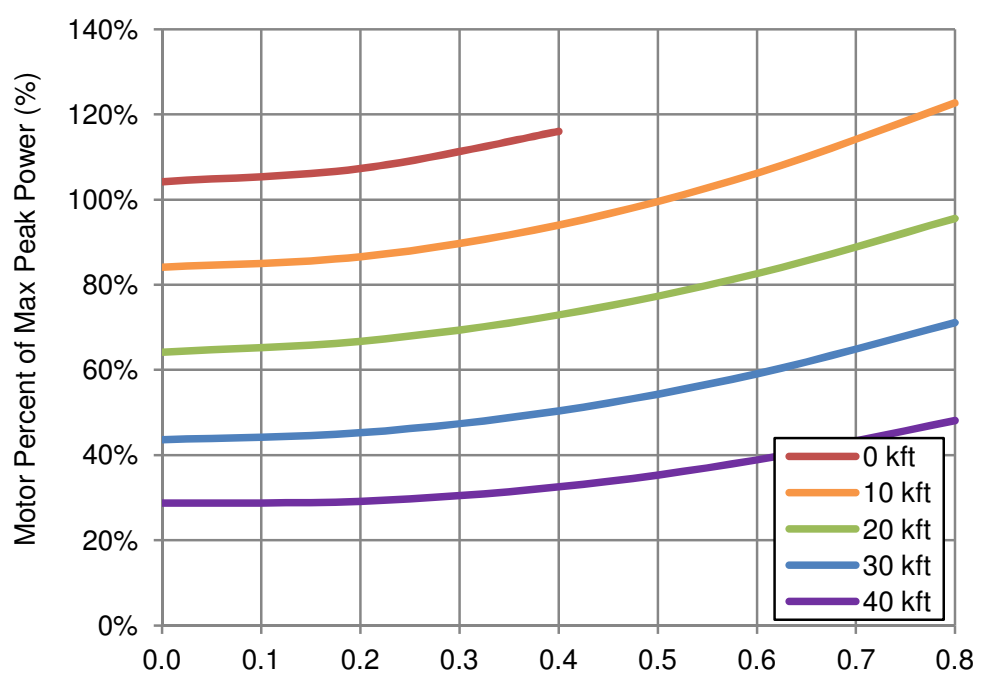

Flight Mach Number

Figure 106. Motor Percent of Max Peak Power versus Flight Mach Number and Altitude. 


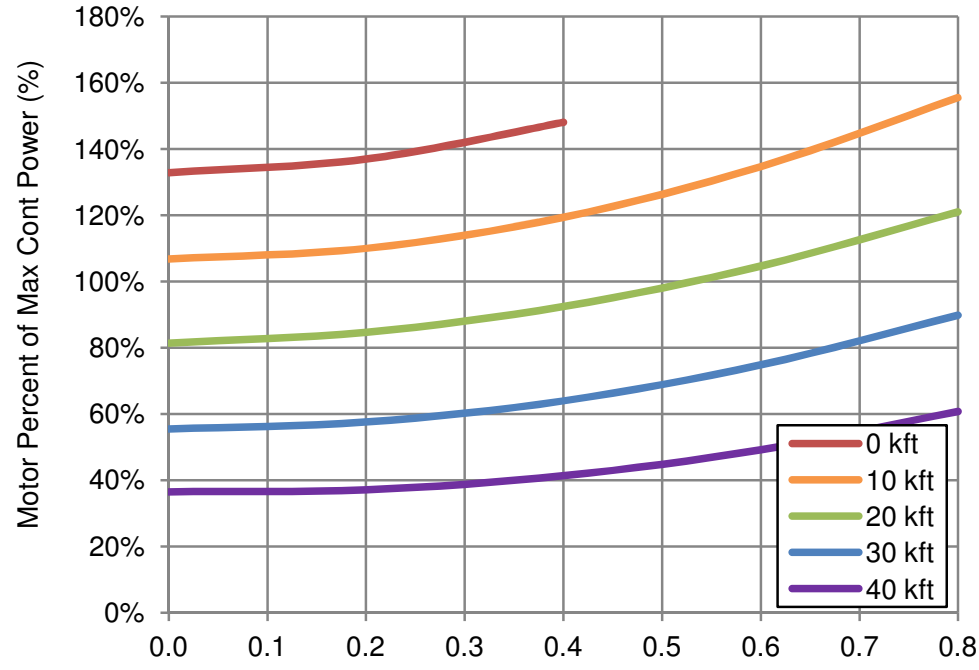

Flight Mach Number

Figure 107. Motor Percent of Max Cont Power versus Flight Mach Number and Altitude.

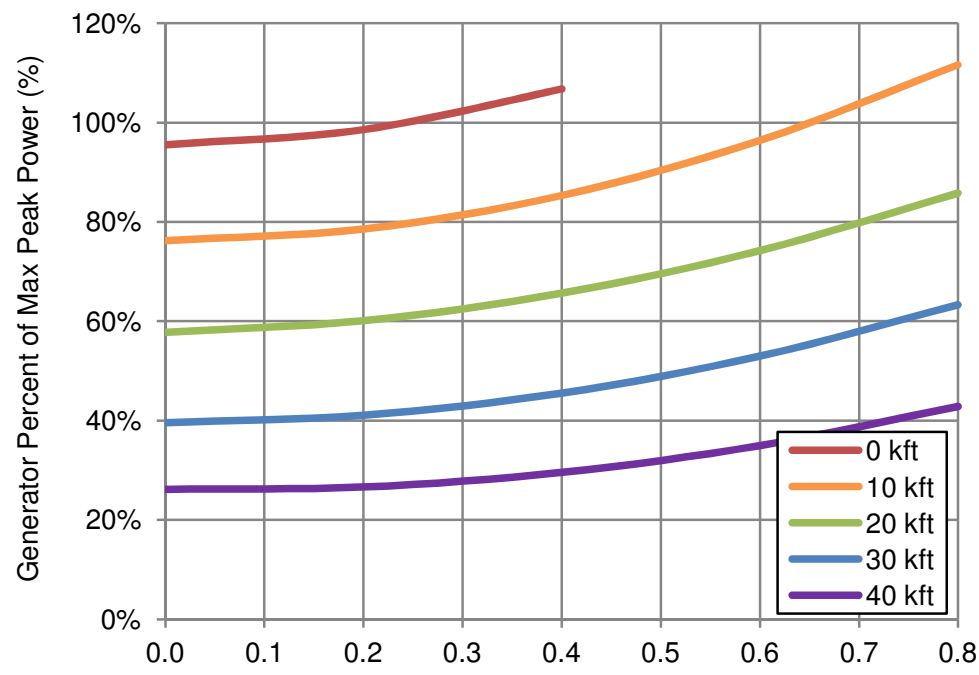

Flight Mach Number

Figure 108. Generator Percent of Max Peak Power versus Flight Mach Number and Altitude. 


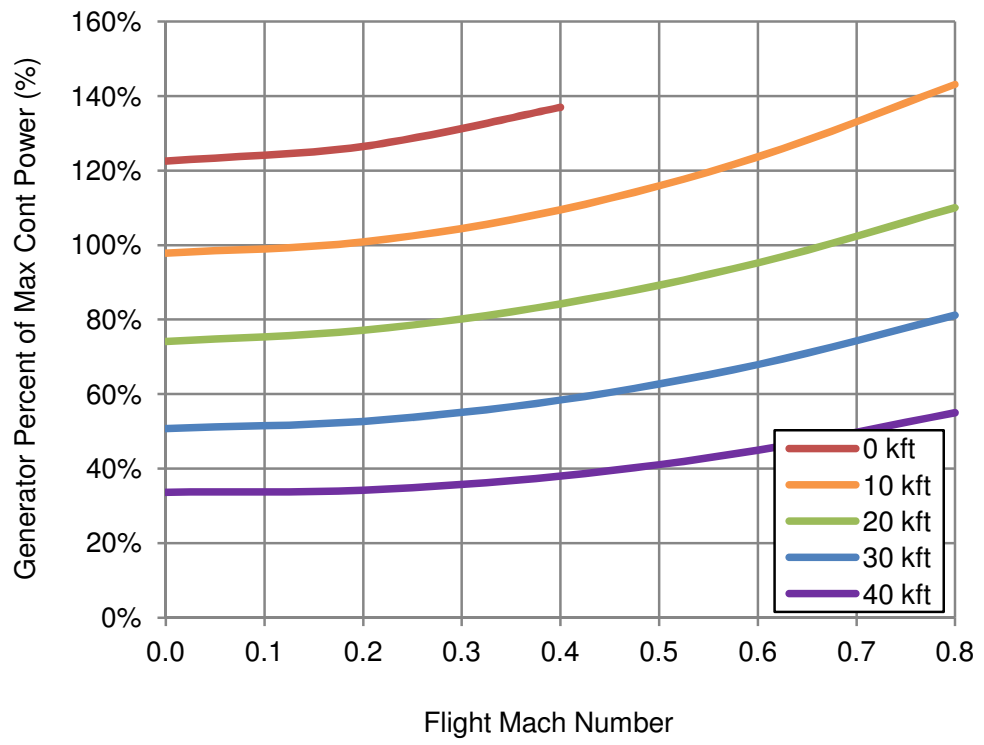

Figure 109. Generator Percent of Max Cont Power versus Flight Mach Number and Altitude.

The trends in these figures again closely match the total engine power figure and the heating curves. At sea level static, the motors are operating as just above $100 \%$ maximum peak power, while the generators are operating just below $100 \%$ maximum peak power. This is important data for the user to consider when sizing the motors and generators as they are only able to sustain maximum peak power for a finite period of time. Above 20,000 feet neither the motors nor the generators will exceed maximum peak power unless supplemental power is provided to the system.

\subsubsection{D Fan Analysis}

The final propulsion system component the user may have interest in are the fans. The off-design performance of the fans is dictated by how much power they can receive from the engines after losses through the electrical system. Figure 110 through Figure 114 show the fan pressure ratio, speed, tip Mach number, face Mach number, and mass flow rate in the off-design domain. 


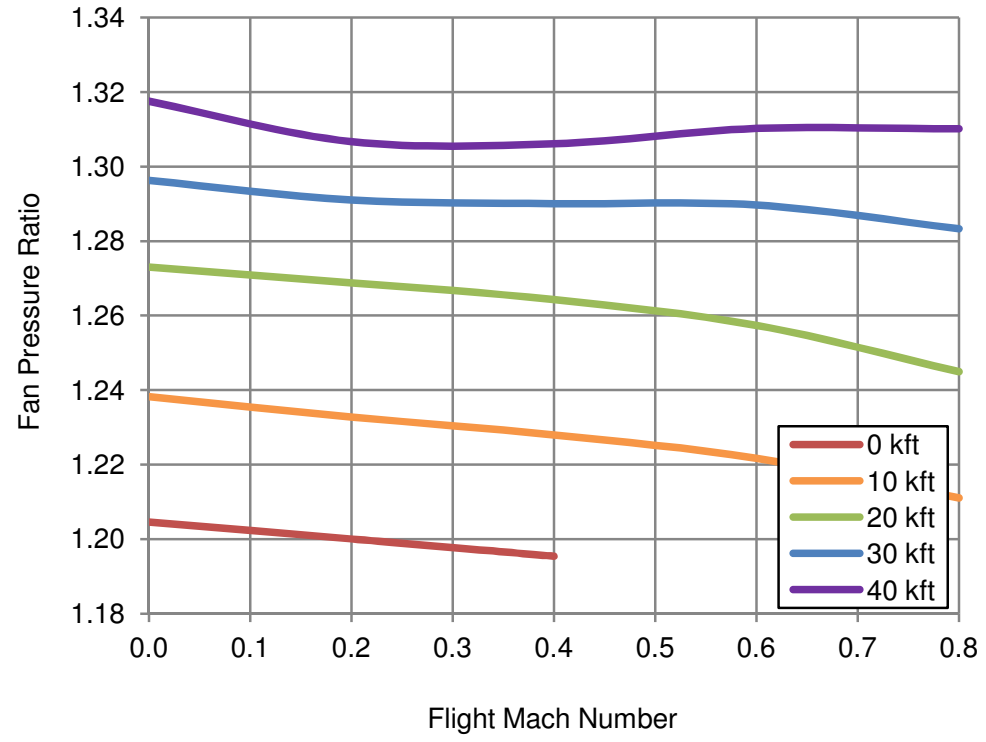

Figure 110. Fan Pressure Ratio versus Flight Mach Number and Altitude.

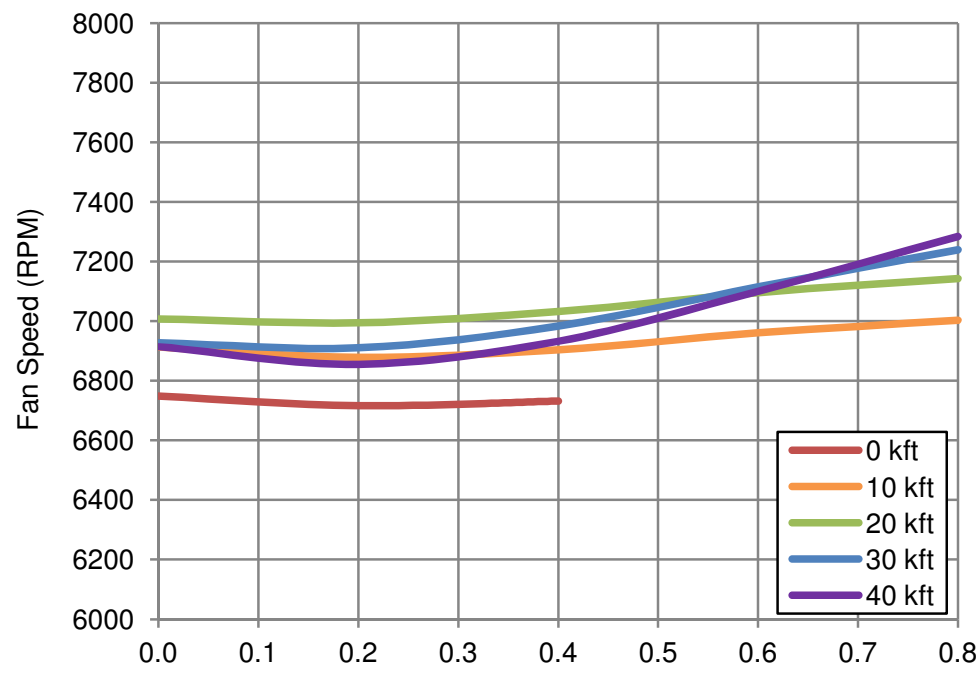

Flight Mach Number

Figure 111. Fan Speed versus Flight Mach Number and Altitude. 


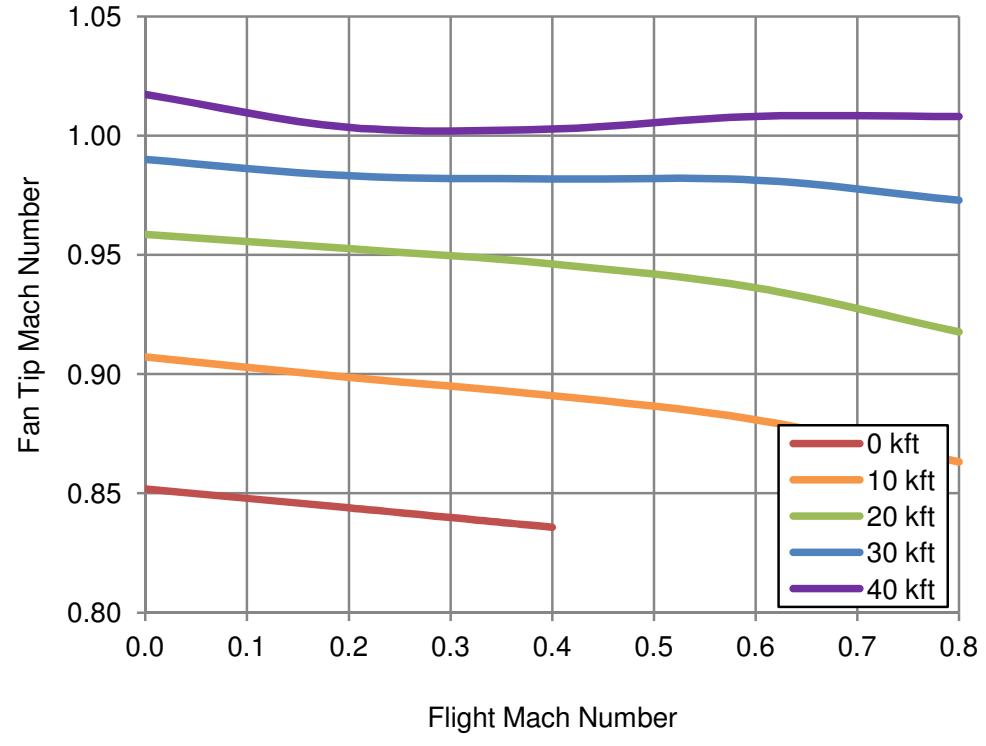

Figure 112. Fan Tip Mach Number versus Flight Mach Number and Altitude.

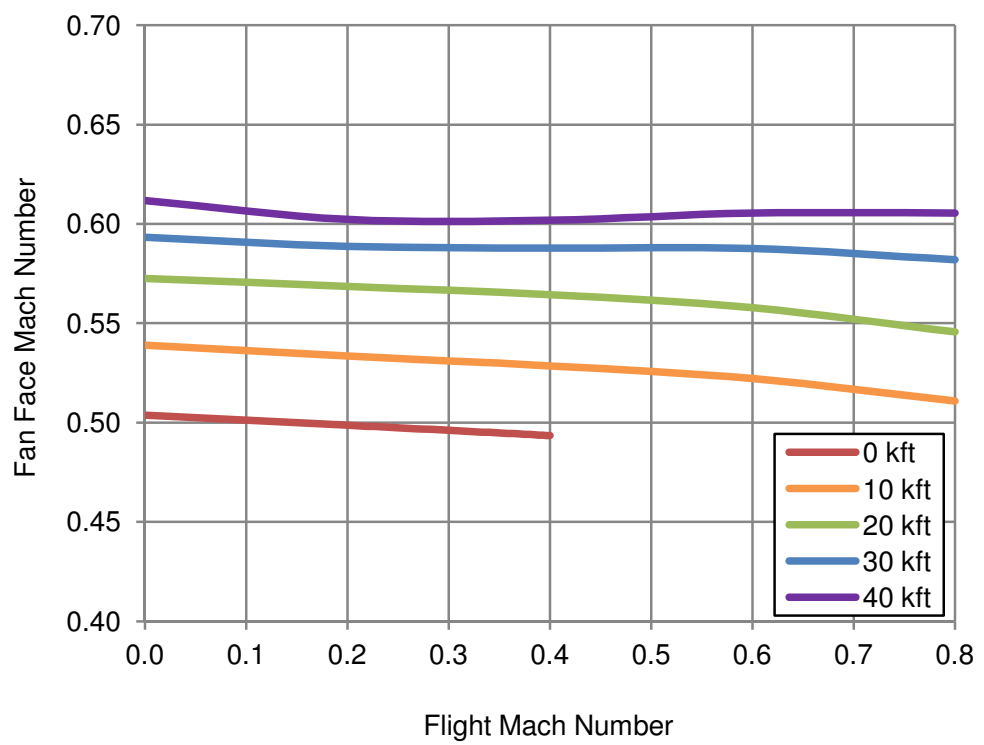

Figure 113. Fan Face Mach Number versus Flight Mach Number and Altitude. 


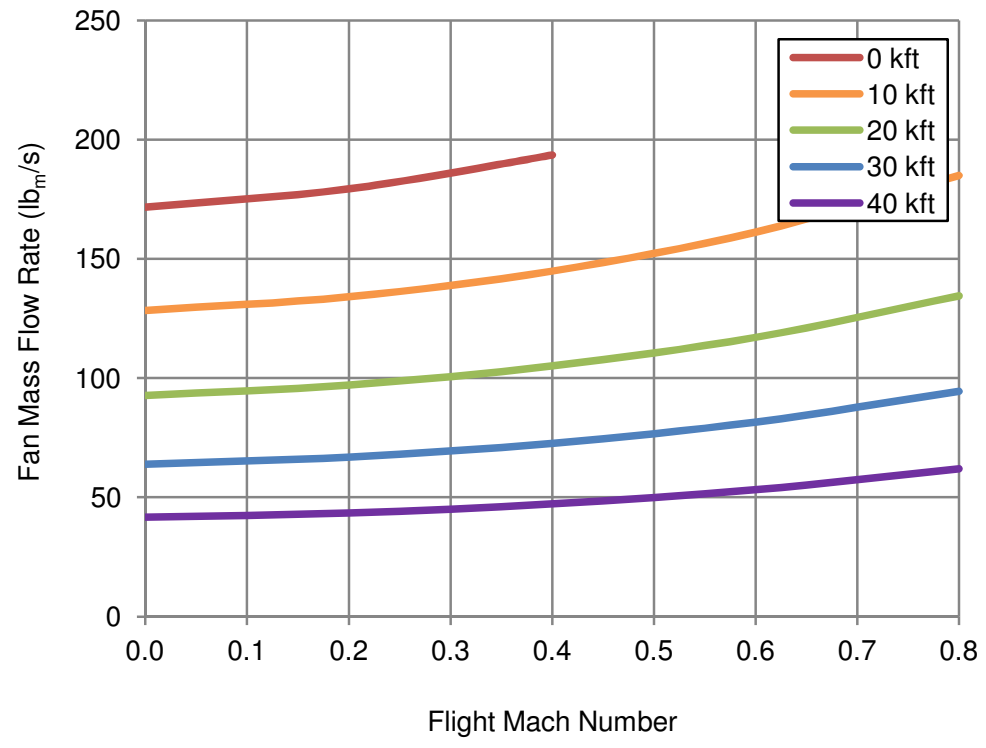

Figure 114. Fan Mass Flow Rate versus Flight Mach Number and Altitude.

The slight variations in the curves of the first four figures are due to the slight variations in the motor and generator efficiencies. The greater the efficiency of the electrical system, the more power becomes available to the fans, increasing fan pressure ratio, speed, and the tip and face Mach numbers.

\subsection{In Conclusion}

This aircraft example was created to show how HAPSS can be used to analyze a hybrid propulsion system for an aircraft. This example also revealed some interesting trends and unique properties of hybrid propulsion systems that the user should be aware of. The trades that were performed in this section are just a small sample of those that the user can consider. These trades were performed using post-processing scripts which are relatively simple to create. Once the user is satisfied with the design of the system and its performance at on and off-design conditions, propulsion system data tables can be produced with HAPSS for use in other mission analysis software. 


\section{Past, Present, and Future of HAPSS}

\subsection{Uses of HAPSS to Date}

Since its inception in early 2010, HAPSS has been utilized for many projects with ESAero. With each project, the HAPSS program has grown from the original 1D fan design tool into a full hybrid propulsion system design and analysis tool with input and feedback from the leaders in the aerospace industry. The following is a list of contract projects for which HAPSS was or is being used for hybrid system analysis. These project descriptions are available in the ESAero company overview $^{\mathrm{xxxv}}$.

- NASA SBIR NNX10CC81P (January 2010 - July 2010)

"Design and Integration of a Distributed Fan Propulsion System within a Split-Wing." Based upon the work of ESAero's previous SBIR effort, ESAero studied the detailed design and integration of a superconducting cryogenically cooled turboelectric Distributed Propulsion system into the split-wing of the ECO-150 configuration. Both the physical considerations of structure and weight, and the aerodynamic considerations of inlet, fan and nozzle design were considered during this study.

\section{- General Atomics, Advanced Technologies Group (June 2009 - July 2011)}

Using SOTA Components in a vehicle configuration down selected during the hybridization planning, a full hybrid system was designed, integrated and analyzed to better refine and assess feasibility and performance improvements. Hybridization design engineering for multiple size and types of vehicles to create and support a technical and marketing plan. Hybridization considered the entire system including electric motors, batteries, controllers, etc. Electric motor focused design and analysis engineering support to General Atomics for the design of multiple hybrid electric air vehicle configurations. Analysis and evaluation of 
General Atomics electric components for insertion into existing and future electric hybrid air vehicles.

- $\quad$ NASA Ames Research Center, SSFW (August 2011 - March 2012)

$\mathrm{N}+2$ electric distributed propulsion aircraft based on the ECO-150 designed during previous SBIR contracts with NASA. ESAero redesigned the aircraft with conventional nonsuperconducting electrical machines. Then, ESAero specifically analyzed the performance of the split-wing configuration for use in the terminal area. This project resulted in articles in the April $2^{\text {nd }}$ and June $4^{\text {th }}$ editions of Aviation Week (see Appendix A).

- The Boeing Company, Boeing Research and Technology (October 2010 - Present) Leveraging the unique capability from ESAero's previous government work in this area, conventional electric machine architectures are being designed and sized for commercial comparison studies. Analysis includes fan design, motor sizing, fan/motor placement and arrangement, weight analysis, and performance prediction.

\section{- Aircraft Design Patent}

Through internal work using the HAPSS code, ESAero has patented an aircraft design.

- $\quad$ AIAA papers for Aerospace Sciences Meeting, Orlando (January 2011)

○ "Split-Wing Propulsor Design and Analysis for Electric Distributed Propulsion"

- $\quad$ AIAA papers for Joint Propulsion Conference, Atlanta (July - August 2012)

- "Analysis of a Distributed Hybrid Propulsion System with Conventional Electric Machines"

- "Benefits and Concerns of Hybrid Electric Distributed Propulsion with Conventional Electric Machines" 


\subsection{Future of HAPSS}

The following is a list of potential additions and modifications to the HAPSS code which would greatly enhance the tool's usefulness, capability, and accuracy.

\subsubsection{D Fan Additions}

- Include basic inlet and nozzle design calculations for determining pressure and velocity losses before and after the fan.

- Implement heat additions to fan exhaust to account for convection cooling of fan motor.

- Add simple losses for stator blades.

- Add blockage factors to account for growing boundary layers in the inlet and exhaust ducts.

- Implement fan performance maps to predict efficiency at off-design conditions.

- Include throttle setting as a design parameter in the fan analysis to better model partthrottle flight conditions such as cruise.

- Possibly model multi-stage and contra-rotating fans.

- Add gearbox model between the fans and motors, including weight and efficiencies.

\subsubsection{D Fan Additions}

- Enhance the 2D blade design analysis with more empirical data for double circular arc airfoils.

- Allow the user to analyze any diameter in 2D instead of just the mean fan diameter.

- Implement the 2D off-design analysis.

\subsubsection{Fan Weight Additions}

- Implement NASA WATE-2 analysis for fan weight estimations.

\subsubsection{Electrical System Additions}

- Further investigate scaling motor and generator weight with torque instead of power, or utilize a fixed power or torque density. 
- Integrate a cable sizing method for estimating the weight, size, lengths, and power losses of the electric cabling.

- Add system voltage, current, frequency, and AC versus DC.

- Create a simple method for estimating the cooling system weight required to cool the electric motors, generators, and controllers.

- Create more performance and efficiency map functions for other high performance motors and generators.

- Add calculation for battery weight and volume required based on power density and energy density.

\subsubsection{Engine Additions}

- Implement a cycle analysis tool to allow the user to adjust the cycle parameters of the engine and to produce detailed engine performance.

- Add gearbox model between the engines and generators, including weight and efficiencies.

- $\quad$ Allow HAPSS to vary engine throttle setting to meet other requirements.

\subsubsection{Miscellaneous Additions}

- Improved nomenclature.

- $\quad$ Add more error reporting to warn user of incorrect inputs or questionable outputs.

- Add the ability to model power removal from the system for environmental control systems and other electrical components.

- Directly link HAPSS with mission analysis software to seamlessly analyze hybrid propulsion configurations on the aircraft level.

In summer 2012, HAPSS will be taking a large step forward with funding from a government entity in conjunction with ESAero to continue the development of the HAPSS program. The intent of this contract is to further develop HAPSS into an advanced hybrid system analysis tool that can be utilized by various members of government. Many of the additions listed 
will be included in this contract work to make HAPSS a powerful and desirable tool for analyzing aircraft hybrid propulsion systems. 


\section{Conclusions}

During the two and a half years that HAPSS has been under development, its usefulness and the need for such an analysis tool has come to light. Hybrid electric propulsions systems, due to their decoupled nature, are notoriously difficult to model by traditional means. Each component in the system can be virtually designed on its own accord since the traditional mechanical linkages are eliminated. This adds many degrees of freedom in the analysis and reveals the countless design approaches that can be investigated.

By decoupling the power producer from the thrust producer, each of these components can operate at or nearer to its optimum efficiency point. This can yield significant increases in thermal efficiency, propulsive efficiency, bypass ratio, ultimately leading to reduced fuel consumption. Unfortunately, the means of decoupling (electricity, gearboxes, mechanical linkages, etc) will ultimately add transmission losses to the overall propulsion system. The goal of HAPSS was to model these losses well and their effect on the system. While the overall efficiency of the hybrid system may be lower than its turbofan counterpart, a decoupled propulsion system can allow the vehicle to fly smarter. An electrically decoupled system, like that modeled by HAPSS, also allow for the use of batteries which add significant flexibility to the design since batteries can supply instantaneous power or absorb excess power at any point during the mission.

Turbofan engines can typically be designed without much knowledge of the aircraft itself other than the basic requirements. A distributed hybrid electric propulsion system should however take into account the aircraft itself, or what is called propulsion airframe integration (PAI). For a distributed propulsion system to be truly advantageous, the propulsive fans must be placed in the airframe such that the vehicle yields aerodynamic, structural, and operational benefits. At this time, HAPSS is unable to model the PAI benefits, but this will hopefully be an addition to the program in the near future. With the addition of batteries, which can be charged or discharged at anytime, the mission that the aircraft is flying also becomes a key design driver of the propulsion system. 
The future of HAPSS looks bright. With so many key players in the aerospace industry investigating aircraft hybrid electric propulsion, a program that can quickly and accurately analyze these complicated systems will be of significant value. And with funding from at least one of these key players, HAPSS will become an even more desirable tool for modeling hybrid aircraft propulsion systems. 


\section{Appendix A: Aviation Week Articles}

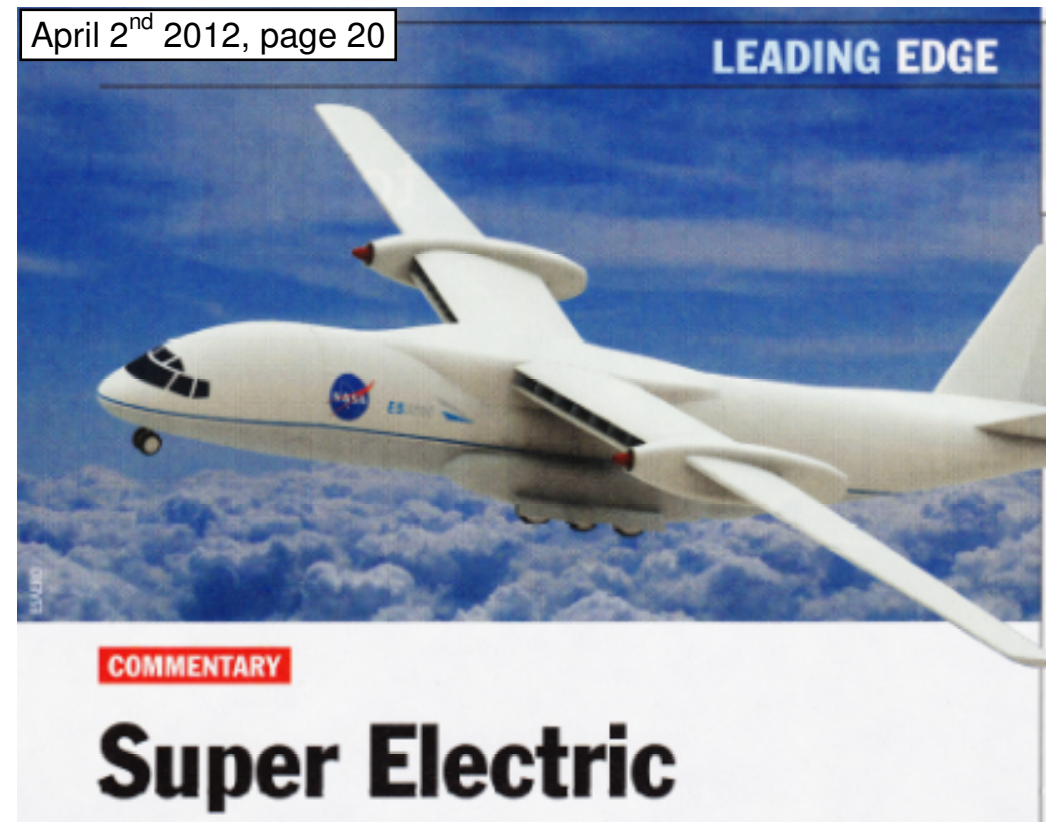

Is superconducting electric propulsion practical?

M ention superconductivity in the context of aircraft propulsion and the skeptics come out in force. But NASA is convinced this technology - still exotic to aerospace - can reduce the fuel consumption and noise of future aircraft.

The agency believes turboelectric distributed propulsion (TeDP) - gas turbines generating electricity to power many small fans embedded in the airframe-can meet its aggressive goals for a 2035-timeframe airliner, slashing fuel burn $60 \%$ from today's Boeing 787s and 777s.

NASA argues cryogenic superconducting components are essential to achieve the efficiencies required to make TeDP viable, but the concept's feasibility may be boosted by a new study suggesting a workable aircraft could be designed around today's room-temperature electrical systems.

Superconducting systems generate far higher magnetic fields than conventional electrical machines, substantially reducing volume and increasing power density. This is essential for aireraft, but means components-generators, motors, inverter/reetifiers and transmission lines - must be cryogeni. cally cooled to minimize losses.

"Superconducting is needed. We have to shrink the size and go after the losses," says Gerald Brown, an engineer at NASA Glenn. "If losses are more than $7 \%$ from the engine to the fan, we may be out of business."
For a 45-kw (60,000-hp) TeDP system powering a 300 -seat hybrid wing body airliner, NASA puts losses at $12 \%$ for ambient-temperature electrics, reducing to $2.1 \%$ with superconductivity. "TeDP at room temperature will not likely save fuel," he says.

But superconducting TeDP is beyond the state of the art. "We have to go beyond today's superconducting motors," which have superconductor $\mathrm{DC}$ windings on the rotor only. The power density needed requires eryogenic superconductor $\mathrm{AC}$ windings on the stator also.

"Superconducting doesn't like AC," says Brown. To minimize losses, winding filaments must be made smaller.

"Most superconducting materials cannot get to the small diameters needed to make a viable machine for aireraft," he says. "But we see one way to go: magnesium diboride seems amenable to making fine filaments suitable for aircraft."

NASA is tangeting a five-fold reduction in weight and low $\mathrm{AC}$ losses in fully superconducting generators and motors. Advanced Magnet Lab is developing a sizing model and will test a fine-filament stator segment to mea-

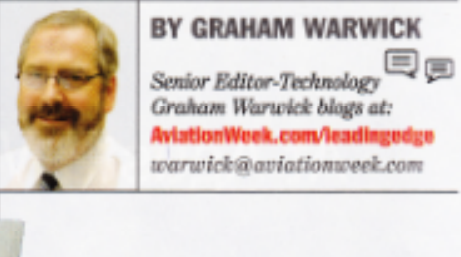

sure the losses. "We think there is a path to a fully supercondueting machine," says Brown.

While liquid hydrogen can be carried to cool the superconductors, NASA is working with Creare Engineering Services to develop a flight-weight cryocooler weighing $5 \mathrm{lb}$./hp-input, down from today's $30 \mathrm{lb} / \mathrm{hp}$. "Cryocoolers exist on spaceeraft, but only at a few watts and we need several kilowatts," he salys, adding: "We see a way to reach the specifle power required."

To minimize distribution losses, $\mathrm{AC}$ rectifiers, $\mathrm{DC}$ transmission lines and DC inverters must also be cooled. Room-temperature invertors are around $97 \%$ efficient, but NASA is working with Mtech Laboratories on cryogenic inverters with $99.5 \%$ efficiency including cryocooler.

NASA is not alone, "There are things driving superconductivity outside aerospace," says Brown. "Utilities are deploying superconducting transmission lines" and the technology could reduce wind-turbine size and power navy warships.

Small advanced-design house ESAero, meanwhile, has produced a 150-seat airliner concept for NASA with liquid hydrogen-cooled superconducting TeDP-fans enbedded in an inboard "split wing" driven by turbogenerators mounted mid-span. Now it has reworked the design (pictured) around room-temperature electrics.

"Our main interest was, could we even get the design to close, and the answer is yes," says ESAero President Andrew Gibson. Performance is about equal to today's 737-700, far short of NASAs goals, but the study sug. gests TeDP could be made to work without pushing the envelope to extremes on superconductivity.

Past studies of turboelectric power concluded it would take motor power densities higher than $10 \mathrm{hp} / \mathrm{lb}$. to make a design work. Current technology is around $4-5 \mathrm{hp} / \mathrm{lb}$. "Technology is not even close to $10 \mathrm{hp} / \mathrm{lb}$., but it appears we do not need that kind of power to close an aircraft," says Ben Schiltgen. ESAero's propulsion system architeet. 


\section{Embedded Power}
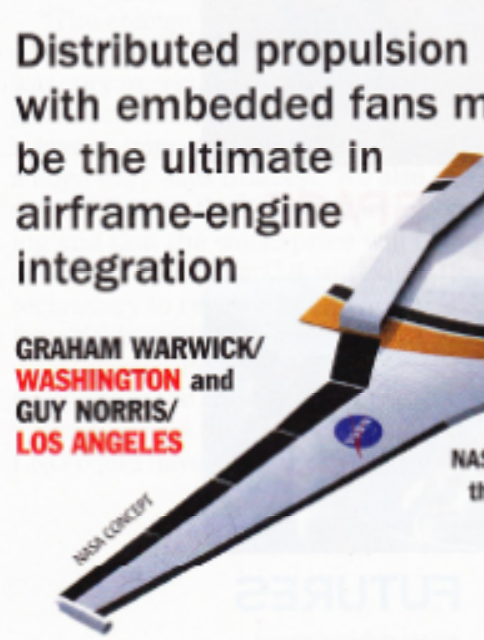

M anufacturers are hanging everlarger engines under the wings of their airliners in the quest for higher bypass ratio and lower fuel burn, and bigger fans lie ahead. Will there come a time in the pursuit of ever-higher efficiency when the trend must reverse?

NASA thinks so, and is pursuing research into distributed propulsion, where many smaller fans are integrated into the airframe so as to not only provide thrust, but to reduce drag and potentially augment lif for short takeoft and perform flight control.

Behind the trend to higher bypass ratios is the basic fact that a given thrust can be produced with less energy by using a langer fan. "But there are challenges in continuing to increase engine diameter, as fans much larger than today's pres ent installation issues, increased weight due to nacelle size, and aerodynamic penalties from increased drag area," says Gerald Brown, senior aerospace engineer at NASA Glenn Research Center:

An array of small fans can have more total area than two large conventional fans, yielding higher bypass ratio and greater propulsive efficiency, he says. If these fans are mounted so they ingest the boundary layer over the airframe and fill in the wake behind the aircraft, there is a major benefit from drag reduction.

For a hybrid wing-body aireraft, up to $8-10 \%$ drag could be eut if enough of the centerbody boundary layer is ingested, Brown says. But individual small engines for each fan could not match the efficiency of large turbofans. Shafts or duets electrical buses, all cryogenically cooled. The combined fan area of the embedded propulsors provides a bypass ratio exceeding 30 and a propulsive efficiency as high as 96\%. "That higher propulsive efficiency more than compensates for the electrical losses in a superconducting power system," says Brown. "If we lose more than $7 \%$ of the energy from engine to fan we may be out of business."

While NASA must significantly reduce the weight and increase the efficiency of superconducting machines to make the concept work, it faces another challenge: To gain the benefit from boundary-layer ingestion (BLI), the fans must work in severely distorted flow. To that end, United Technologies Research Center (UTRC) is developing a distortion-tolerant fan.

“We started a program several years ago to address the propulsion aspects of BLI. Previous work in this area had addressed the vehicle-level benefits, but we wanted to focus on the effect on the turbomachinery," says Greg Tillman, principal engineering scientist for Pratt \& Whitney programs at UTRC.

When work began, NASA's hybrid wing-body concept had deeply embedded engines with S-duct intakes, but studies showed the losses in a highly offset inlet duct could wipe out the benefit from BLI. "So we started with a clean sheet and evolved a different design with a much more modest inlet offset, to minimize the negative hit on inlet pressure losses and fan efficiency," he says. propulsors via superconducting motor; generators, transformer/rectifiers and

\section{Shaft-drive or gas-drive - too complex?}

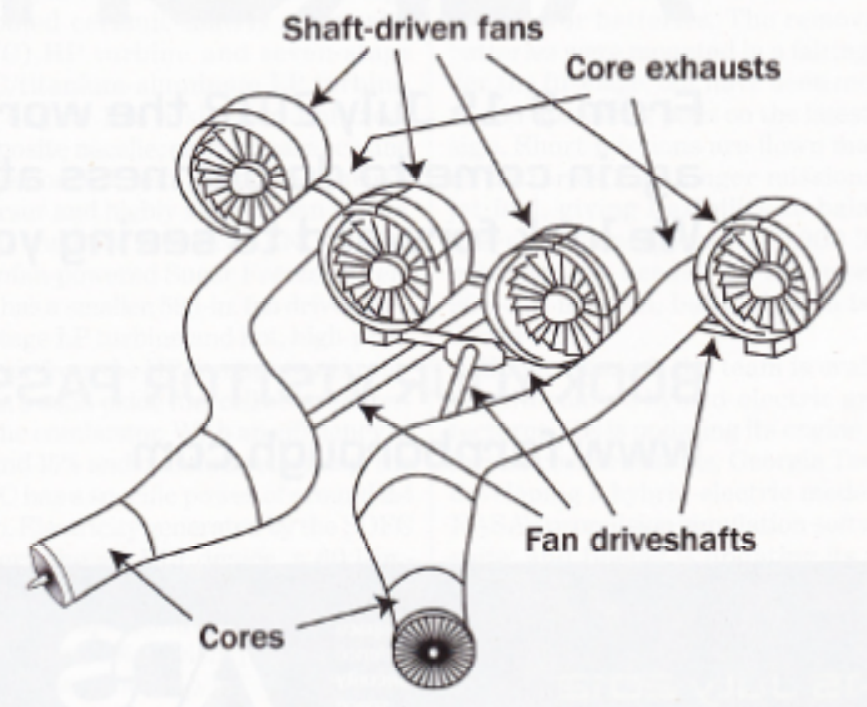

62 AVIATION WEEK \& SPACE TECHNOLOGY/JUNE 4/11, 2012

AvintionWeek.com/awst 


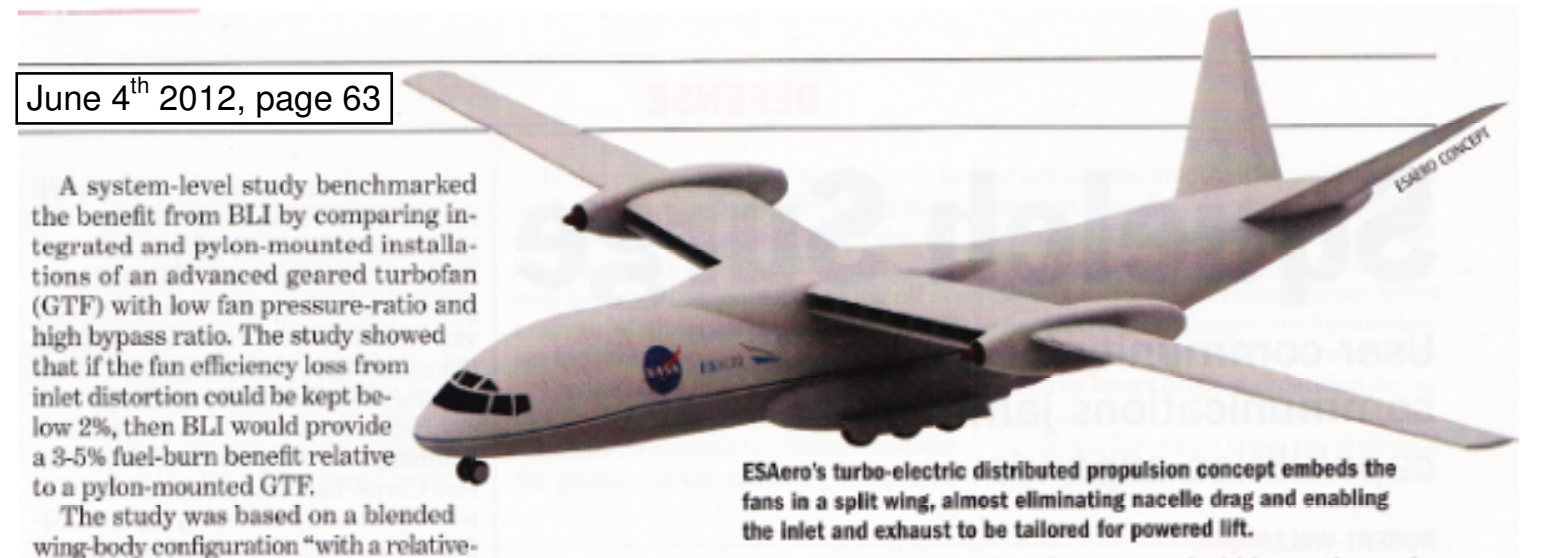
wing-body configuration "with a relatively modest amount of boundary layer that could be ingested over the upper center fuselage," he says. "The BLI benefit scales with the amount of viscous drag ingested versus engine thrust. If you integrate the engines to ingest more boundary layer, you increase the benefit."

Embedded fans lose 5-6\% efficiency in distorted flow, says $\mathrm{Om}$ Sharma, UTRC senior fellow. "Our goal is to reduce this to a couple of percent, so we can really reduce the wake strength and provide better propulsive efficiency." As the fan rotates through distorted flow, incidence on the blades changes. Some areas of the rotor see more flow and ehoke; others see less flow and stall. The fan will incur high dynamic stresses due to aeroelastic forced-response and possibly flutter. "The adverse effects are pretty large, and over a large area-20-30\% change of lift on a blade, across $30 \%$ of the inlet height," says Tillman. "If we didn't do something about it, it would break the fan."

The original plan was to modify the fan, but the solution lay in coupled design of the inlet, fan and exit guide vanes (EGV). "We came in thinking we would end up with a fan-centric design. We discovered a complete inlet/tan/EGV system is the essence of how to deliver a distortion-tol. erant fan with the required performance, operability and aeromechanies," he says.

The inlet is modified to "redistribute the harmonic energy in the distortion coming into the fan into places in the spectrum where interaction with the fan is favorable," he says. This involves spreading the energy content, lowering the penks and moving it to higher harmonics that will not excite destructive structural vibration of the blades.

Initially, UTRC looked at deflecting the boundary layer away from the fan, "but that did not taloe advantage of BLI to improve propulsive efficiency," says Sharma. Active inlet flow-control was considered, but rejected. "It did not give the

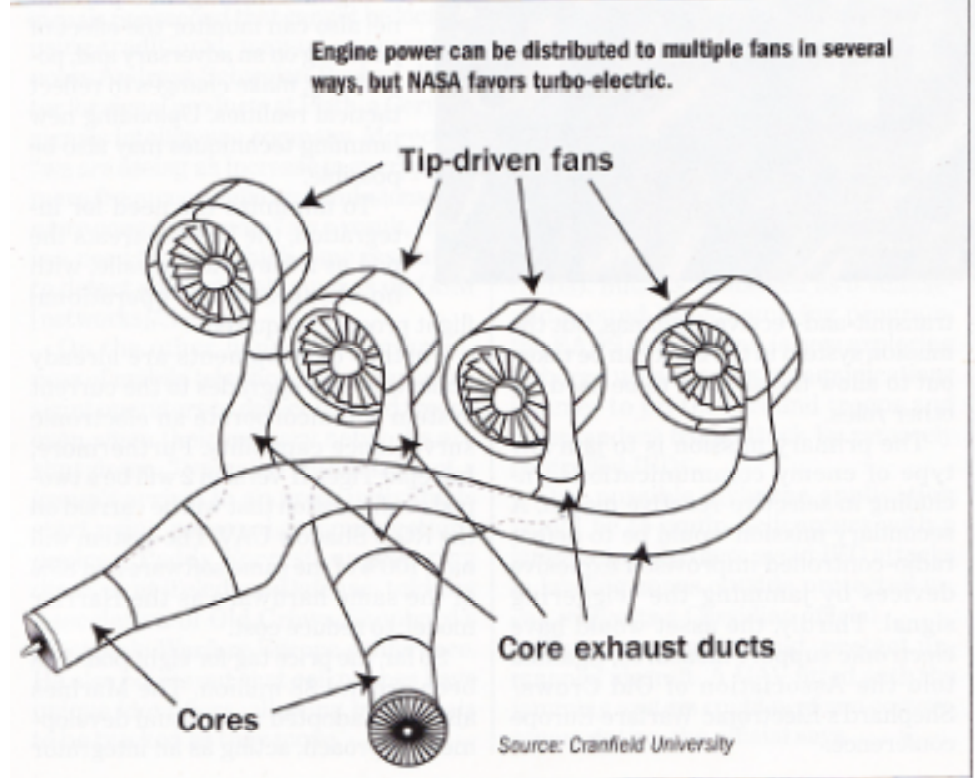

benefit we wanted within our timescale. Maybe in the future it will play a bigger role," he says. "It's difficult for active flow control to buy its way in, so we went with a passive system," says Tillman.

UTRC is not saying much about the distortion-tolerant fan itself. "The improvement comes not from particular magic in the fan, but from careful design of a fully coupled system," he says, "If you step back, it does not look much different." But the exit guide vanes do, and are key to the design as they must cope with distorted flow that leaves the fan. "EGV: are designed to operate in distortion and are non-axisymmetric," says Tillman. UTRC is building a scale model of the integrated inlet and fan for tests in the $8 \times 6$ - $\mathrm{ft}$. wind tunnel at Glenn. "The hardware should be fabricated and delivered in the second quarter of fiscal 2013 , and the test is planned for the third quarter," says Ruben Del Rosario, NASA Subsonic Fixed Wing program manager. Although blended wing-body transports with turbo-electrie distributod propulsion using boundary-layer ingestion lie decades in the future, UTRC's distortion-tolerant fan work could have nearer-term application to the GTF, says Alan Epstein, Pratt \& Whitney vice-president for technology and environment.

"At Pratt, we see bypass ratios go up and up. Inlets will have to get shorter [to reduce weight/dragl, and they can. Today, most of the inlet is used to absorb noise. As we make fan pressure-ratio lower and bypass ratio higher, the fan turns slower and makes less noise, so we need less inlet," he says. "The bad news is that's less inlet for distortion attenuation."

Pratt's interest in distortion-tolerant fans is for conventional inlets on 13-18:1 bypass-ratio GTFs on conventional configurations in the late 2020 s/early $2030 \mathrm{~s}$. "It's a good example of UTRC's work on advanced embedded-engine distributed propulsion having much nearer-term applieation directly lined up with Pratt's business strategy," Epstein says, ○ 


\section{Bibliography}

'Lemieux, Joe. "Design Challenges of Hybrid and Fuel Cell Electronics." Design Challenges of Hybrid and Fuel Cell Electronics. EE Times, 19 Aug. 2003.

ii Warwick, Graham. "Fuel-Sipper - the Turbo-Electric Flying Wing." Aviation Week 25 May 2011.

iii Kim, Hyun D. Distributed Propulsion Vehicles. Tech. Cleveland, OH: NASA Glenn Research Center.

iv Empirical Systems Aerospace. The Design and Integration of a Distributed Fan Propulsion System within a Split-Wing. NASA SBIR NNX10CC81P, Phase 1 Report, 2009. Unpublished

$\checkmark$ "The Turbofan Engines." IKFUPM.com. 2010. <http://www.ikfupm.com/forum/showthread.php?366-DS®The-Turbofan-Engines>.

vi "The Technology Behind the CFM56-7B Turbofan Engine." CFM.

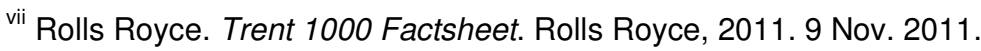

viii'Honeywell. TPE331-10 Turboprop Engine. Phoenix, AZ: Honeywell, July 2009.

${ }^{i x}$ United States. Federal Aviation Administration. Federal Aircraft Regulations Part 25 Sec. 25.121.

${ }^{x}$ Empirical Systems Aerospace. <http://www.esaero.com/>.

${ }^{x i}$ MATLAB. Computer software. Help Documentation. Vers. R2010a. Mathworks.

xii Vanco, Michael R. Computer Program for Design-Point Performance of Turbojet and Turbofan Engine Cycles. Tech no. TM X-1340. NASA Lewis Research Center, 1967.

xiii McBride, Heimel, Ehlers, and Gordon. Thermodynamic Properties to 6000K for 210 Substances involving the First 18 Elements. Tech no. SP-3001. NASA Lewis Research Center, 1963.

xiv U.S. Standard Atmosphere, 1976, Government Printing Office, Washington, DC, 1976.

${ }^{x v}$ Zucrow, Maurice J., and Joe D. Hoffman. Gas Dynamics. New York: Wiley, 1976.

xvi Pera, Ornat, Klees, and Tjonneland. A Method to Estimate Weight and Dimensions of Aircraft Gas Turbine Engines. Tech. no. CR135170. NASA Lewis Research Center, 1977.

xvii Hale. A Method to Estimate Weight and Dimensions of Small Aircraft Propulsion Gas Turbine Engines, Final Report and User's Guide. Tech no. CR168049. NASA Lewis Research Center, 1982.

xviii Cohen, Henry, G. F. C. Rogers, and H. I. H. Saravanamuttoo. Gas Turbine Theory. Burnt Mill, Harlow, Essex, England: Longman Scientific \& Technical, 1987.

xix Lieblein. Review of High-Performance Axial-Flow-Compressor Blade-Element Theory. Tech no. E53L22. NACA Lewis Flight Propulsion Laboratory, 1954.

${ }^{x x}$ GEnx Engine Fan Blade. Digital image. GE Aviation. GE.

xxi GE90 Composite Fan Blade. Digital Image. GE Reports. GE, 2012.

xxii Carter. The Low Speed Performance of Related Airfoils in Cascades. Tech no. R.55. Ministry of Supply, Aeronautical Research Council, 1949.

xxiii Schwenk, Leiblein, and Lewis. Experimental Investigation of an Axial-Flow Compressor Inlet Stage Operating at Transonic Relative Inlet Mach Numbers. Tech no. E53G17. NACA Lewis Flight Propulsion Laboratory, 1953. 
xxiv Lewis, and Schwenk. Experimental Investigation of a Transonic Axial-Flow-Compressor Rotor with Double-Circular-Arc Airfoil Blade Sections. Tech no. E54J08. NACA Lewis Flight Propulsion Laboratory, 1955.

${ }^{x x v}$ Remy International. Remy HVH250-090-SOM Electric Motor. Remy International, 2011.

xxvi Aerodynamic Design of Axial-Flow Compressors. Tech no. SP-36. NASA Lewis Research Center. 1965.

xxvii Mort. Performance Characteristics of a 4-Foot-Diameter Ducted Fan at Zero Angle of Attack for Several Fan Blade Angles. Tech no. TN D-3122. NASA Ames Research Center, 1965.

xxviii Lewis, and Schwenk. Experimental Investigation of a Transonic Axial-Flow-Compressor Rotor with Double-Circular-Arc Airfoil Blade Sections. Tech no. E53L21a. NACA Lewis Flight Propulsion Laboratory, 1954.

xxix Klapproth, Jacklitch, and Tysl. Design and Performance of a 1400-Foot-Per-Second Tip-Speed Supersonic Compressor Rotor. Tech no. E55A27. NACA Lewis Flight Propulsion Laboratory, 1955.

${ }^{x x x}$ Lieblein, Schwenk, and Broderick. Diffusion Factor for Estimating Losses and Limiting Blade Loadings in Axial-Flow-Compressor Blade Elements. Tech no. E53D01. NACA Lewis Flight Propulsion Laboratory, 1953.

xxxi Brown, Gerald V., Hyun D. Kim, and James Felder. "Materials Aspects of Turboelectric Aircraft Propulsion." 2009 Annual Meeting, Fundamental Aeronautics Program, Subsonic Fixed Wing Project. Sept.Oct. 2009. Presentation.

xxxii Gallimore, Simor J., John J. Bolger, Nicholas A. Cumpsty, Mark J. Taylor, Peter I. Wright, and James M. Place. "The Use of Sweep and Dihedral in Multistage Axial Flow Compressor Blading - Part I: University Research and Methods Development." ASME Journal of Turbomachinery 124 (2002): 521-32.

xxxiii Kerrebrock, Jack L. Aircraft Engines and Gas Turbines. Cambridge, MA: MIT, 1977.

xxxiv Cumpsty, N. A. Jet Propulsion: A Simple Guide to the Aerodynamic and Thermodynamic Design and Performance of Jet Engines. 2nd ed. Cambridge: Cambridge UP, 1997.

xxxv Empirical Systems Aerospace, Inc, Company Overview. Empirical Systems Aerospace, Inc. <http://esaero.com/ESAero\%20Company\%20Overview.pdf> 